\title{
Dissecting mechanisms of neonatal cardiac regeneration
}

Citation for published version (APA):

Sampaio Pinto, V. (2021). Dissecting mechanisms of neonatal cardiac regeneration. [Doctoral Thesis, Maastricht University]. ProefschriftMaken. https://doi.org/10.26481/dis.20210608vp

Document status and date:

Published: 01/01/2021

DOI:

10.26481/dis.20210608vp

Document Version:

Publisher's PDF, also known as Version of record

\section{Please check the document version of this publication:}

- A submitted manuscript is the version of the article upon submission and before peer-review. There can be important differences between the submitted version and the official published version of record.

People interested in the research are advised to contact the author for the final version of the publication, or visit the DOI to the publisher's website.

- The final author version and the galley proof are versions of the publication after peer review.

- The final published version features the final layout of the paper including the volume, issue and page numbers.

Link to publication

\footnotetext{
General rights rights.

- You may freely distribute the URL identifying the publication in the public portal. please follow below link for the End User Agreement:

www.umlib.nl/taverne-license

Take down policy

If you believe that this document breaches copyright please contact us at:

repository@maastrichtuniversity.nl

providing details and we will investigate your claim.
}

Copyright and moral rights for the publications made accessible in the public portal are retained by the authors and/or other copyright owners and it is a condition of accessing publications that users recognise and abide by the legal requirements associated with these

- Users may download and print one copy of any publication from the public portal for the purpose of private study or research.

- You may not further distribute the material or use it for any profit-making activity or commercial gain

If the publication is distributed under the terms of Article $25 \mathrm{fa}$ of the Dutch Copyright Act, indicated by the "Taverne" license above, 


\title{
Dissecting mechanisms of neonatal cardiac regeneration
}

\author{
Vasco Sampaio Pinto
}

$\mathrm{PhD}$ Thesis Co-Supervision between

the University of Porto and the University of Maastricht 
(C) copyright Vasco Sampaio Pinto, Maastricht 2021

Cover Design: Vasco Sampaio Pinto \& Anna Maria Altrocchi

Printing: ProefschriftMaken || www.proefschriftmaken.nl

ISBN/EAN: 978-94-6423-277-6

All rights reserved. No part of this publication may be reproduced, stored in a retrieval system or transmitted, in any form or by any means, electronic, mechanical, photocopying, recording or otherwise, without prior permission of the author or the copyright-owning journals for previous published chapters. 


\title{
Dissecting mechanisms of neonatal cardiac regeneration
}

\author{
Dissertation
}

to obtain the degree of Doctor at Maastricht University, and Doutor em Ciências Biomédicas at the ICBAS - University of Porto on the authority of the Rector Magnifici,

Prof. dr. Rianne M. Letschert and Prof. dr. António Sousa Pereira, in accordance with the decision of the Board of Deans, to be defended in public on Tuesday, $8^{\text {th }}$ June 2021 , at 10.00 hours

by

Vasco Sampaio Pinto 


\section{Supervisors:}

Dr. Diana dos Santos Nascimento (University of Porto)

Prof.dr. Paula A. da Costa Martins (Maastricht University)

\section{Co-supervisor:}

Dr. Perpétua Pinto-do-Ó (University of Porto)

\section{Assessment Committee:}

Prof. dr. Lorenzo Moroni (Chair, Maastricht University)

Prof. dr. Judith Sluimer (Maastricht University)

Dr. Linda van Laake (UMC Utrecht)

Prof. dr. Maria Oliveira (University of Porto)

Prof. dr. Mauro Giacca (King's College London)

This thesis was financed by Norte Portugal Regional Operational Programme (NORTE 2020), under the PORTUGAL 2020 Partnership Agreement, through the European Regional Development Fund(ERDF) (NORTE-01-0145-FEDER-000012); by European Structural and Investment Funds (ESIF), under Lisbon Portugal Regional Operational Programme and National Funds through FCT (Fundação para a Ciência e Tecnologia [Foundation for Science and Technology] [POCI-01-0145-FEDER-016385] and [POCI-01-0145-FEDER-030985]); by INFARMED - Autoridade Nacional do Medicamento e Produtos de Saúde, I.P. (FIS-FIS-2015-01_CCV_20150630-157); and by FCT/Ministério da Ciência, Tecnologia e Inovação in the framework of the project "Institute for Research and Innovation in Health Sciences" (POCI-01-0145- FEDER-007274) and individual fellowship: SFRH/BD/111799/2015 to V.S.-P. 


\section{Table of Contents}

CHAPTER 1

General Introduction $\quad 5$

CHAPTER 2

Non-coding RNAs in endothelial cell signaling and hypoxia during cardiac regeneration 23

$\begin{array}{ll}\text { CHAPTER } 3 & 45\end{array}$

Neonatal apex resection triggers cardiomyocyte proliferation, neovascularization and functional recovery despite local fibrosis $\quad 45$

$\begin{array}{ll}\text { CHAPTER } 4 & 85\end{array}$

Stereological estimation of cardiomyocyte number and proliferation $\quad 85$

$\begin{array}{ll}\text { A stereological perspective on cardiac ontology } & 103\end{array}$

$\begin{array}{ll}\text { CHAPTER } 5 & 111\end{array}$

Summary \& General Discussion 111

$\begin{array}{ll}\text { IMPACT } & 121\end{array}$

$\begin{array}{ll}\text { ABSTRACT IN PORTUGUESE } & 125\end{array}$

$\begin{array}{ll}\text { ACKNOWLEDGEMENTS } & 127\end{array}$

$\begin{array}{ll}\text { BIOGRAPHY } & 131\end{array}$

LIST OF PUBLICATIONS 131 



\title{
CHAPTER 1
}

\section{General Introduction}

Adapted from:

\section{Cardiac regeneration and repair: from mechanisms to therapeutic strategies}

\author{
Vasco Sampaio-Pinto ${ }^{1,2,3,4,5}$, Ana C. Silva ${ }^{1,2,3,6}$, Perpétua Pinto-do-Ó, ${ }^{1,2,3}$, Diana S. \\ Nascimento ${ }^{1,2,3}$
}

${ }^{1}$ i3S - Instituto de Investigação e Inovação em Saúde, Universidade do Porto, Porto, Portugal;

2 INEB - Instituto Nacional de Engenharia Biomédica, Universidade do Porto, Porto, Portugal;

${ }^{3}$ ICBAS - Instituto de Ciências Biomédicas Abel Salazar, Universidade do Porto, Portugal;

${ }^{4}$ Department of Cardiology, CARIM School for Cardiovascular Diseases, Faculty of Health, Medicine and Life Sciences, Maastricht University, Maastricht, The Netherlands;

${ }^{5}$ IMAIA - Institute of Molecular Biology and RNA Technology, Faculty of Sciences and Engineering, Maastricht University, Maastricht, The Netherlands;

${ }^{6}$ Gladstone Institutes, University of California San Francisco, San Francisco, USA.

Springer book - concepts and applications of stem cell biology: a guide for students doi 10.1007/978-3-030-43939-2

ISBN: 978-3-030-43939-2 



\section{Epidemiology of Cardiovascular Disease}

Cardiovascular diseases (CVD) are the leading cause of death worldwide. Epidemiological reports from 2013 showed that globally CVD were responsible for approximately $32 \%$ of total deaths (i.e. 17 million) (1). An update showed that, in 2016, 45\% of deaths in Europe (i.e. 4 million) were attributed to CVD (2). Although there is some genetic contribution, most risk factors associated with heart diseases are behavioural and environmental, such as age, gender, high blood pressure, hypercholesterolemia, smoking, obesity and alcohol consumption (3). As a result, CVD are more prevalent in high-income countries.

Amongst CVD, ischemic heart diseases are the most predominant (Figure 1). Ischemia in the heart, as in the case of myocardial infarction (MI), generates a hypoxic environment that ultimately leads to cell death. Owing to the negligible regenerative capacity of the human heart, upon $\mathrm{Ml}$, the damaged myocardium is replaced by a fibrotic scar, resulting in progressive deterioration of organ function and development of heart failure.

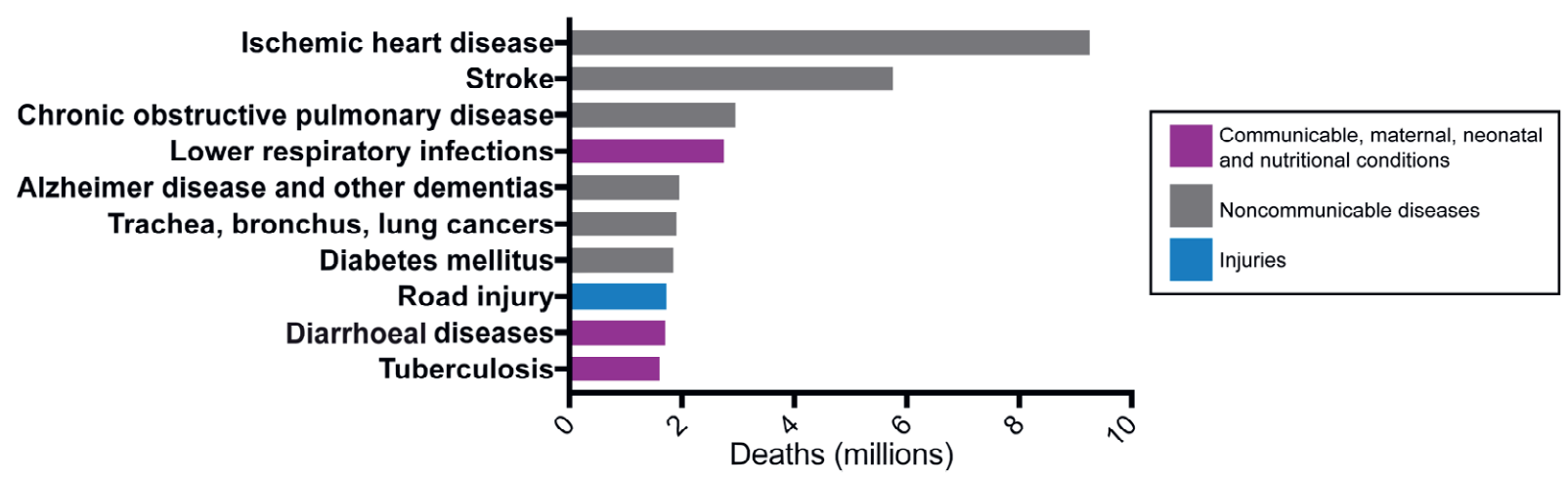

Figure 1 - Top 10 global causes of deaths, 2016

(Adapted from Global Health Estimates 2016: Deaths by Cause, Age, Sex, by Country and by Region, 2000-2016. Geneva, World Health Organization; 2018)

Currently, heart failure drugs and interventions target disease progression and, thus, the only efficient treatment to re-establish the injured muscle is whole-organ transplantation, which is limited by the shortage of donors, organ rejection/failure and side effects related to immunosuppression (4).

The scarcity of alternative therapies prompted the cardiovascular community to search for novel regenerative mechanisms by dissecting the molecular machineries regulating heart development and response to injury. Because regeneration of the human adult myocardium is virtually inexistent, the heart has been classically considered a post-mitotic organ. By the turn of the century, evidence of cardiomyocyte renewal throughout human life (5) and after MI (6), questioned this paradigm. In fact, the prospect of cardiomyocyte proliferation after birth, even at rates incompatible with regeneration, sustained the hypothesis that the heart is endowed with intrinsic capacity for de novo muscle formation that could be exploited to promote a better outcome after injury. 


\section{Evolutionary perspective on cardiac development, physiology and injury- response}

The heart of vertebrates displays unique features distinguishable from invertebrate species as is the case of highly specialized chambers with internal endothelial lining (reviewed in (7)). However, substantial differences regarding cardiovascular anatomo-physiology are observed across vertebrates, most likely a result of independent processes of environmental adaptation.

The first evidence of adult heart regeneration in lower vertebrates was obtained in zebrafish after surgical resection of the apex (10-20\% of the ventricle). In this model, the cardiac tissue is progressively restored within 60 days (8).

Initially a blood clot is formed which is gradually replaced by newly formed cardiomyocytes, restoring organ contractility to original levels. Genetic tracing of cardiomyocytes further confirmed that de novo cardiomyocyte formation encompasses dedifferentiation and proliferation of pre-existing cardiomyocytes (9).

Recent evidences highlight similarities between cardiomyocytes of adult lower vertebrates and foetal mammalian cardiomyocytes, which are yet immature (Figure 2).

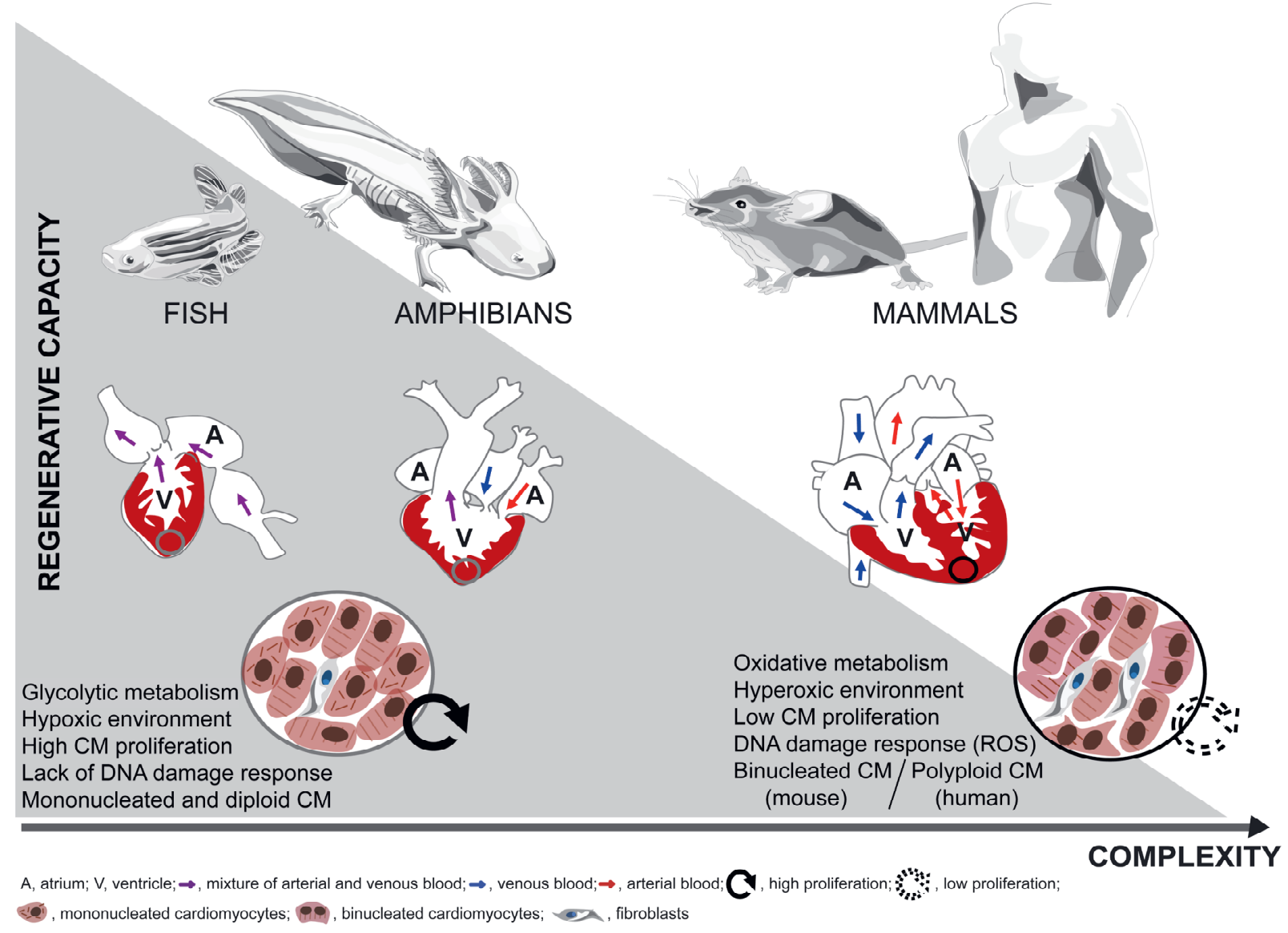

Figure 2 - The phylogenetic axis of cardiac complexity versus regenerative capacity. 
Whereas adult zebrafish and foetal mammalian cardiomyocytes display glucose-based metabolism and high proliferative capacity $(10,11)$, post-natal mammalian cardiomyocytes reside in an oxygen-rich environment, generate energy mainly through oxidative metabolism and have withdrawn from the cell-cycle $(10,12)$. Also, zebrafish cardiomyocytes alike foetal mammalian cardiomyocytes are mononucleated, diploid and lack markers of DNA damage response, which contrasts with the binucleation and polyploidy, high levels of mitochondrial reactive oxygen species and DNA damage response observed in adult mammalian cardiomyocytes $(10,13)$.

Globally, these comparative studies demonstrate that biochemical and anatomical complexity of the cardiovascular system across phylogeny is inversely correlated with the regenerative capacity of the heart (Figure 2).

\section{An ontogenic window for cardiac regeneration in mammals}

The first reference to heart regeneration in mammals was provided by Mario Robledo in 1956. In this study, myocardial lesions by cauterization were shown to induce cardiomyocyte cell division (14). Remarkably, the idea of a developmental window for heart regeneration in mammals only became an established principle 55 years later, following the work by Sadek and colleagues (15). Hearts of 1-day old mice (P1) after surgical apex resection progressively re-established the amputated region with negligible fibrosis. However, when the same procedure was performed at P7, hearts failed to regenerate. Hence, while proliferation of pre-existing cardiomyocytes was the main regenerative mechanism at $\mathrm{P} 1$, response at $\mathrm{P} 7$ resembled that of the adult heart, characterized by fibrosis without evidence of cardiomyocyte proliferation (15).

In addition to apex resection, other models showed the regenerative capacity of neonatal mice at various extents (i.e. cryoinjury, genetic ablation of cardiomyocytes, ligation of left anterior descending (LAD) coronary artery, ischemia/reperfusion (I/R) and pressure overload by transverse aortic constriction (TAC)). Interestingly, cryoinjury at P1 was typically associated with incomplete regeneration, yet depending on injury severity.

In humans, evidence suggests that the heart of children and infants may exhibit regenerative capacity. In the first half of the last century, analysis of post-mortem histological heart sections of children with diphtheria highlighted the presence of split myofibers and mitotic cells in the myocardium which were interpreted as a result of cardiac regeneration $(16,17)$. Other studies showed that neonatal hearts were able to fully recover from spontaneous Ml occurring after birth $(18,19)$. These findings support that also human young hearts preserve the ability to regenerate.

The work by Sadek and colleagues (15), transformed the cardiovascular field and has been fully acknowledged by over 900 citations. However, in 2014, controversy was settled when Andersen and colleagues, while attempting to establish the neonatal apex resected model, reported a complete absence of cardiac regeneration (20). In opposition to the pioneer work, these authors documented a strong inflammatory response in the injury site, followed by massive collagen deposition. Additionally, resected hearts were shown to be 
smaller than the respective surgical controls, exhibiting impaired neovascularization and cardiomyocyte proliferation. On a later follow up study, the same authors showed that the scarring tissue resulting from the apex resection was permanently deposited in the myocardium and that these animals developed dilated cardiomyopathy (21).

The explanation for these disparate results is still not fully understood (22-24) but some progress was made in the subsequent years. Injury severity was shown to impact on the development of fibrosis both after apical resection (25) and cryoinjury (26). Moreover, difficulties associated with the assessment of fibrosis deposition and cardiomyocyte proliferation can preclude an accurate interpretation of the biological events occurring after injury.

\section{From regeneration to repair - ontogenic changes during neonatal life}

In mammals, the reactivation of neomyogenic mechanisms upon injury is quickly diminished after birth although the mechanisms that govern this shift of potential are still incompletely understood. During intra- to extra-uterine environmental transition, mammalian hearts undergo dramatic alterations that mark the end of cardiac morphogenesis and the beginning of myocardial maturation (Figure 3). A better understanding of tissue dynamics during this fundamental transition will certainly reveal cues for the development of innovative therapies.

\section{Cardiomyocytes}

The mammalian heart develops from a simple contractile tube to a four-chambered organ. At late developmental stages, the heart is still a hyperplastic organ, composed by a highly proliferative population of mononucleated cardiomyocytes with little organized sarcomeres that support the final stage of myocardium growth $(7,27)$ (Figure 2 and Figure 3).

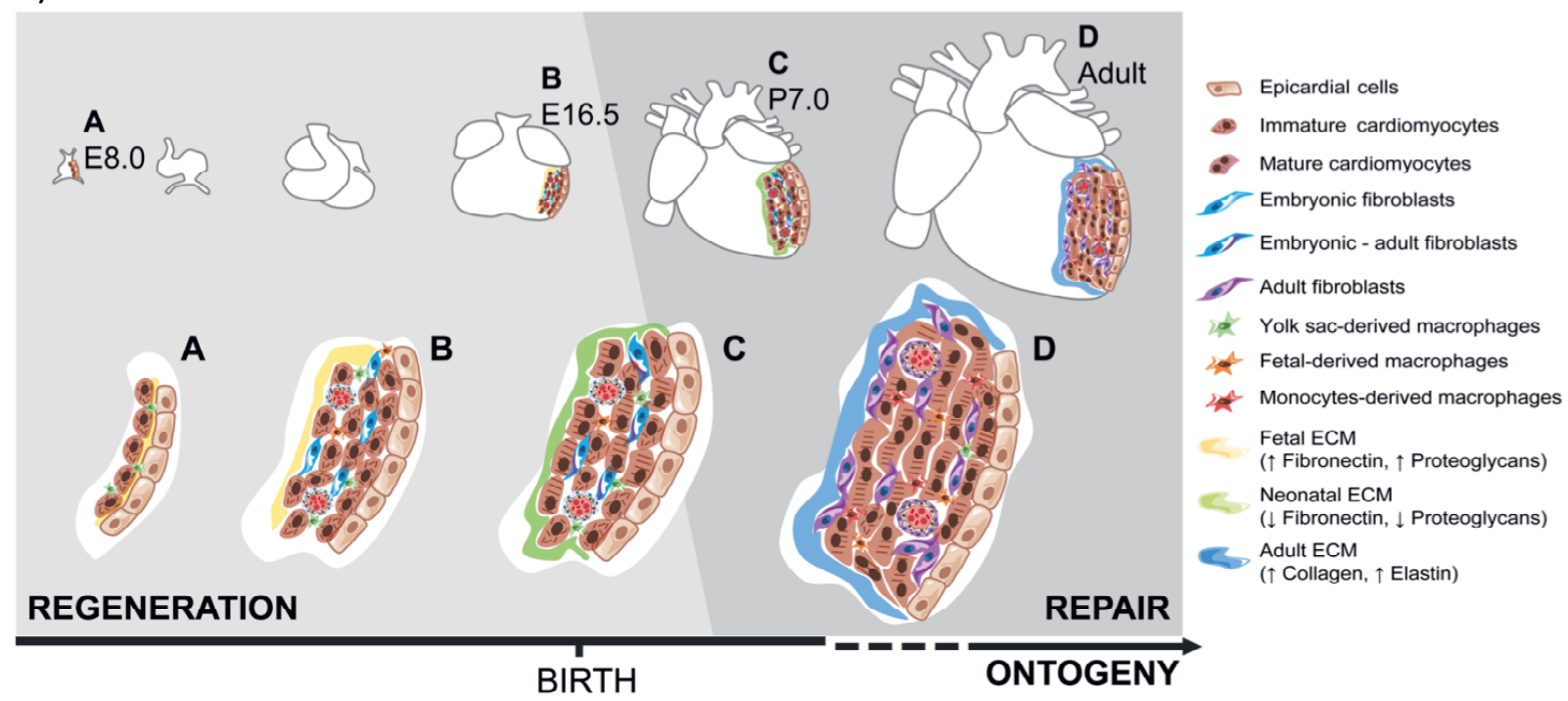

Figure 3 - Schematic perspective of cardiac tissue cellular and extracellular dynamics and regenerative capacity throughout ontogeny. 
At birth, in order to meet systemic requirements of extra-uterine life, the mammalian heart undergoes profound physiologic and metabolic remodelling. Blood oxygenation starts in the lungs and closure of the extra- and intra-cardiac shunts suddenly increases oxygen pressure and systemic vascular resistance (28).

At this stage, cardiomyocytes, that during development produce energy mainly by anaerobic glycolysis and lactate oxidation, undergo a permanent shift towards aerobic metabolism, while retaining for a short period the unique ability to resist to ischemia and hypoxia (29). More impressively, correlation between this metabolic change and arresting of cell cycle activity has been recently reported. Puente and colleagues precisely timed the metabolic switch in the mouse heart from anaerobic glycolysis to mitochondrial oxidative phosphorylation to the first week after birth. Indeed, reactive oxygen species (ROS), a byproduct of mitochondrial respiration formed during ATP synthesis, are responsible for the activation of DNA damage response pathways, triggering cardiomyocyte withdrawal from the cell cycle (10). In line with this, an elegant study by Nakada et al. showed that exposure to gradual systemic hypoxaemia rescued infarcted hearts of more severe remodelling while leading to new myocardium at expenses of pre-existing cardiomyocyte proliferation (30). Apart from upstream environmental factors aforementioned, several molecular regulators of postnatal cardiomyocytes cell cycle arrest have been identified and can fall within one of three levels of regulation: 1) epigenetic regulation involving pre-transcriptional heterochromatin-mediated gene silencing of positive cell cycle regulators; 2 ) transcriptional activation of negative cell cycle regulators and 3 ) post-transcriptional regulation through non-coding RNAs (31).

A cell intrinsic timer mechanism was shown to limit the proliferation of rat neonatal cardiomyocytes by the activation of cyclin-dependent kinases (CDK) inhibitors (32). In fact, the activity of cyclin $\mathrm{E}$ - and cyclin A-associated CDK complexes is severely reduced around P5 (33). Furthermore, knockdown of the CDK inhibitors p21 and p27 in adult cardiomyocytes induced cellular proliferation, highlighting a putative role for these elements in cell cycle exit (34). In addition, by overexpressing of CDK1, CDK4, cyclin B1 and cyclin D1, Mohamed and colleagues were able to induce stable cell division of post-mitotic mouse, rat and human cardiomyocytes (35).

After birth, cardiomyocytes silence cardiac development-related genes (i.e. Notch and Wnt/b-catenin pathways) and cell cycle progression genes via hypermethylation (36). In contrast, overall hypomethylation is detected in genes involved in heart contractility (i.e. myosin heavy-chain, titins and troponin I) and mitochondrial activity (37). Major epigenetic changes are set until P14 (36) although a partial reactivation of foetal development program was documented in response to pathological conditions $(37,38)$.

Identification of miRNAs involved in cardiac disease has led to the study of their involvement also in heart development and cardiac regeneration. Knock-out of miRNA133a induced proliferation of cardiomyocytes through de-repression of cyclin D2 (39). Similarly, miR-15 family was found to target checkpoint kinase 1 (Check1) and thus, this miRNA knockout increased cardiomyocyte proliferation (40,41). Inversely, miR-199a-3p and miR-590-3p induced cell cycle re-entry of cardiomyocytes by inhibiting Homer1 and 
Hopx, a regulator of calcium signalling in cardiac cells and a repressor of embryonic myocytes proliferation, respectively (42). In fact, overexpression of miR-199a in pig hearts subjected to $\mathrm{Ml}$ stimulated cardiomyocyte proliferation and improved cardiac repair (43). Moreover, knockdown of anti-mitotic genes meis1, miR-548c-3p, miR-509-3p and miR-23b$3 p$ promoted cardiomyocyte proliferation (44).

Recent reports also suggest that, besides miRNAs, other non-coding RNAs can regulate cardiomyocyte proliferation. The long non-coding RNA (InCRNA) CAREL was found to be upregulated in non-mitotic cardiomyocytes and its overexpression inhibited neonatal cardiac regeneration by supressing cardiomyocyte proliferation (45).

\section{Non-cardiomyocyte heart cell subsets}

The inflammatory response following injury, such as $\mathrm{MI}$, has been extensively characterized. Despite the well-recognized importance of macrophages on cardiac healing, the heterogeneity of subsets, developmental origin and activation upon injury was only recently scrutinized. Cardiac resident macrophages, mainly derived from the yolk sac (primitive haematopoiesis, E7.5-E11.5) and foetal liver (definitive haematopoiesis, E11.5E16.5), were shown to persist until adult life (Figure 3). After injury these embryonic macrophages are permanently replaced by infiltrating monocyte-derived macrophages (46). Importantly, clodronate-based depletion of macrophages during the neonatal period, abrogated heart regeneration and led to the development of fibrotic scars with impact on cardiac contractility and angiogenesis. An independent study, using an inducible transgenic mouse to enable cardiomyocyte depletion, explored further the role of distinct macrophage lineages in neonatal and adult response to injury. Whereas in the neonate following injury the number of resident cardiac macrophages increases with negligible monocyte recruitment, the adult heart engages on a vigorous immune response with higher levels of inflammatory cytokines and chemokines, strong monocyte recruitment and frequent monocyte-derived macrophages (47). Importantly, inhibition of monocyte recruitment showed to be sufficient to reduce inflammation, protect the pool of resident embryonicderived macrophages and, consequently, to improve repair of the heart (47).

In the past, fibroblasts have been mostly regarded as a cellular type with minor physiological and developmental importance which was readily activated in pathological conditions to orchestrate a vigorous reparative response culminating in the formation of fibrosis. Currently, fibroblasts are gathering attention as bi-directional cardiomyocytefibroblast interactions became extensively reported (48). leda et al. elegantly demonstrated that fibroblasts change with age, especially in the transition between intra- and extrauterine life, having thus distinctive roles over cardiomyocytes. Opposing to adult fibroblasts, embryonic fibroblasts secrete predominantly growth factors, such as, heparinbinding endothelial growth factor (HBEGF), an important trigger of cardiomyocyte proliferation, and ECM components crucial for cardiac development (i.e. fibronectin, tenascin-C, periostin, hyaluronan and collagen) (49). Adult fibroblasts exhibit a 58-fold 
increase in the expression of IL-6, a cytokine recognized to induce cardiomyocyte hypertrophy $(49,50)$ (Figure 3).

Endothelial cells were also shown to contribute to the early response of neonatal hearts to injury. Capillaries migrate into the apical thrombus as soon as 2 days after apical resection and undergo maturation into fully functional arteries within 5 days. This event precedes cardiomyocyte proliferation and is required for neomyogenesis (51). Moreover, arterial endothelial cells were shown to migrate along pre-existing capillaries and form collateral arteries following neonatal MI. Such event increases reperfusion of the ischemic myocardium and allows efficient cardiac regeneration (52).

In addition, resorting to the pharmacological, genetic or mechanical inhibition of cardiac innervation both in zebrafish and neonatal mouse, Mahmoud et al. proved that cardiac denervation impacts negatively on cardiomyocyte proliferation and inflammatory response hindering cardiac regeneration. Interestingly, administration of recombinant neuregulin 1 and Nerve growth factor partially rescued the regenerative capacity of the heart (53).

\section{$\underline{\text { Extracellular cardiac microenvironment }}$}

The cardiac ECM is a dynamic network composed by a diversity of molecules, namely collagens, non-collagenous glycoproteins (e.g. fibronectin and laminin) and proteoglycans that are essential for tissue integrity but also in coordinating biological processes during heart morphogenesis, homeostasis and pathology (54).

During heart development, ECM consists on a dynamic network enriched in fibronectin, proteoglycans and growth factors (55-57) (Figure 3). The early matrix influences the initial peristaltic pumping of the cardiac tube, the regulation of epithelial-mesenchymal transition (EMT), which is responsible for the formation of cardiac valves and atrioventricular/outflow septa $(58,59)$ and myocardial growth $(49)$. The composition of ECM molecules undergoes profound reduction postpartum, being progressively replaced by a collagen and elastin rich meshwork $(55,60,61)$. In fact, collagen deposition and crosslink during cardiac tissue maturation are essential for normal cardiac performance by supporting the alignment of cardiac fibres, cardiomyocyte-fibroblast bridging and electrical conductance $(62,63)$.

ECM derived from distinct ontogenic stages impact differently on cardiac cells behaviour. Through the use of ontogenic-tissue-specific ECM coatings, Williams and colleagues demonstrated that foetal cardiac ECM promotes cardiomyocytic expansion in vitro when compared with the adult counterpart (61). Subsequently, decellularized foetal ECM showed greater potential to support and instruct cardiac cells in comparison to adult-derived ECM (64).

\section{Past, present and future of therapeutic approaches}

Besides the deteriorating impact on life expectancy and quality, CVD also impose a heavy socio-economic burden. Encouragingly, the cardiovascular community is strongly determined in developing innovative and more effective therapeutic strategies (Figure 4). 


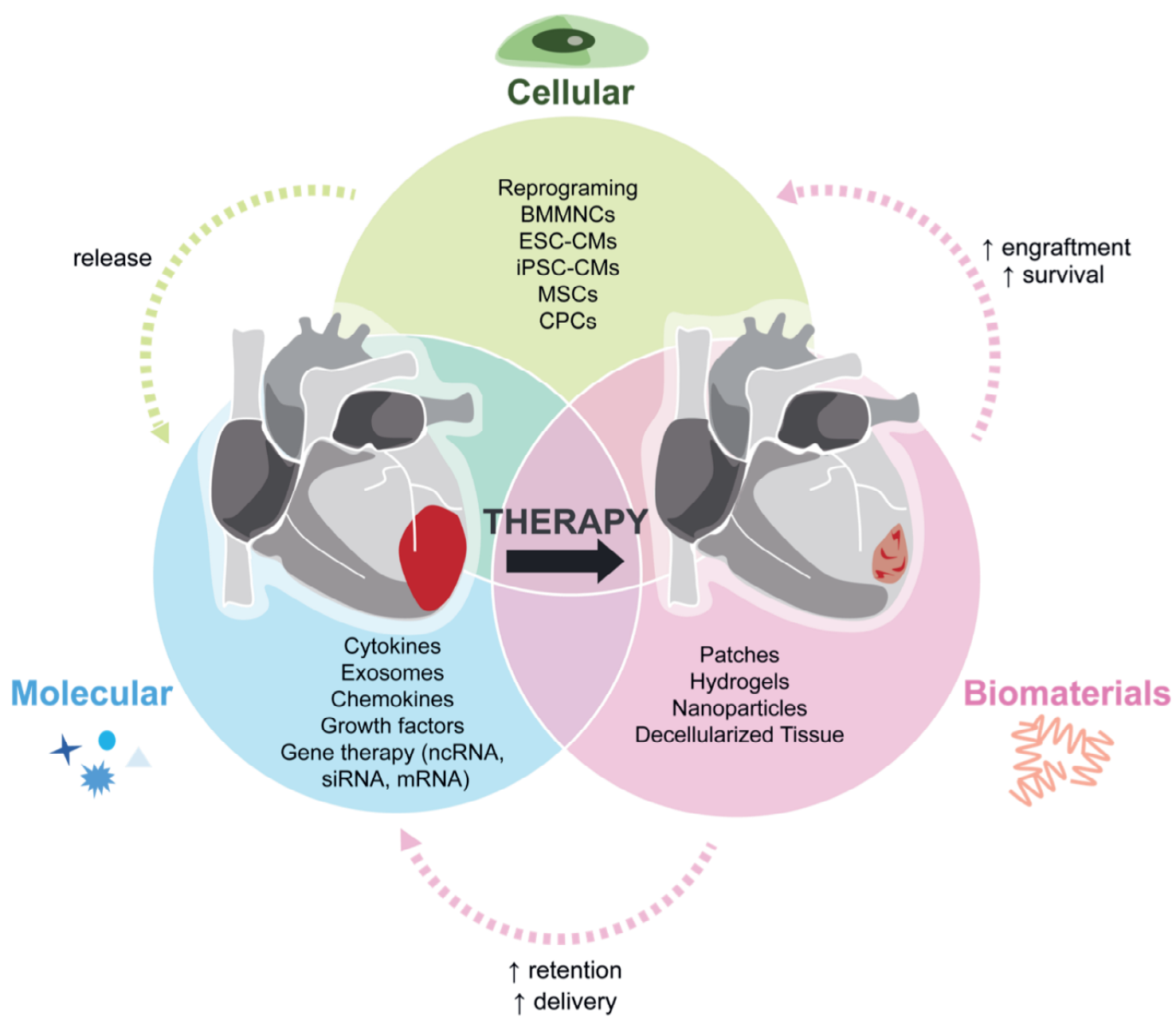

Figure 4 -Therapeutic approaches to treat myocardial infarction.

Neomyogenesis is a prime objective after MI. In an initial approach, the attempts resorted to skeletal myoblasts, however transplantation of autologous skeletal myoblasts significantly increased the risk of developing ventricular arrhythmias (65). Bone marrow mononuclear cells (BMMNCs) were also on the first-line for heart therapies (66-68). However, likely due to poor engraftment and differentiation after transplantation, clinical trials using bone marrow-derived cells to treat MI proved disappointing (69). In addition, mesenchymal stem cells (MSCs) isolated from the bone marrow and from sources such as adipose tissue and earlier ontogenic sources (e.g. Wharton's jelly), were pursued for the treatment of heart failure patients due to their potential to secrete growth factors and their immunomodulatory properties. These features hold therapeutic value and endorse mesenchymal cells for allogenic transplantation $(70,71)$. Of note, although clinical reports are promising this far (72), larger and controlled trials are required to validate further the efficiency of MSCs. Remarkably, the cardiac function improvement has been accredited to a paracrine action rather than to direct differentiation into cardiomyocytes.

By the turn of the century, cells exhibiting stem cell-like properties were identified in the adult heart of rodents and humans. These cells, frequently referred to as "cardiac stem cells" (CSCS) or "cardiac progenitor cells" (CPCS), were identified by the expression of 
distinct cell surface markers (i.e. proto-oncogene receptor tyrosine kinase (c-Kit), stem cell antigen 1 (Sca-1), ATP-binding cassette super family G (Abcg-2) and platelet-derived growth factor receptor $\alpha$ (PDGFR- $\alpha$ )) and transcription factors (e.g. Isl-1, Nkx2.5, Gata-4 and Wilms' tumour $1(\mathrm{Wt}-1))(73,74)$. However, the early excitement on the evidence for these cells to differentiate in cardiomyocytes and endothelial cells in vitro and in vivo was promptly challenged by a series of studies, some incorporating lineage tracing, who could neither replicate the findings nor show putative CSC/CPC contribution to de novo cardiomyocyte formation (75-77). Hence, whether or not renewal on the adult myocardium is based on stem cells became a contentious topic in the cardiovascular area. Meanwhile, CPCs had gathered the biomedical community interest and at least two trials addressing different subsets of progenitor cells (c-Kit ${ }^{+}$for SCIPIO (78) and Sca- $1^{+}$for CADUCEUS (79)) were launched to test the therapeutic potential on the patients' infarcted heart. Deplorably, ethical concerns on the research conducted by the main contributors to the endogenous c$\mathrm{Kit}^{+} \mathrm{CSCs}(80)$ were raised over the last years overshadowing science $(81,82)$ and bringing the concept in the twilight.

Pluripotent stem cells, i.e. embryonic stem cells (ESC) and iPSCs are, when stimulated by a defined cocktail of growth factors, the only cells that display robust differentiation into functional cardiomyocytes thus constituting the most promising cell source for cardiac regenerative therapy (Figure 4). Even so, ESC- and iPSC-derived cardiomyocytes display functional and morphological features of immature myocytes thereby resembling foetal cardiomyocytes (83-86). Promising increments emerge by the day as the efforts of the experimental cardiovascular community succeed on, for example, identifying transcriptional regulatory networks directing transition from the immature neonate to a mature cardiomyocyte stage (87). In addition, human ESC-derived cardiomyocytes implanted into non-human primates after Ml showed to increase muscle mass of the heart. Interestingly, autologous muscle coupled electrically with the graft that was also irrigated by the vasculature of the host (88). In a recent clinical case, ESC-derived cardiac progenitors, embedded in a fibrin scaffold, were transplanted into the infarcted area of a patient with advanced ischemic heart failure. Three months after administration of the cells the symptomatology of the patient improved while complications such as arrhythmias, tumour formation or immunosuppression-related adverse events were not described (89).

Nevertheless, despite the extensive enterprise to develop a cell-based therapy, myocardial cell delivery commonly results in poor engraftment, cell death or cell loss into the bloodstream and subsequent entrapment in the lungs and liver $(90,91)$. Several distinct strategies have been considered to improve cell retention, survival and coupling in the heart (92), including the use of decellularized cardiac ECM (64), injectable hydrogels (93), cardiac patches (94) and piezoelectric biomaterials that promote the alignment and stimulation of cardiomyocytes (95) (Figure 4).

Because functional improvement of cell therapy appears to rely on paracrine mechanisms, new strategies are focusing on replacing cells by soluble factors (e.g. chemokines, cytokines and growth factors). However, the administration of molecules with putative beneficial effects (e.g. vascular endothelial growth factor (VEGF), granulocyte-colony stimulating 
factor (G-CSF) and erythropoietin (Epo) produced none or small functional increment after MI (96-98), which may be related with inappropriate dosage or organ selectivity.

Owing to their ability to regulate gene expression, miRNAs and IncRNAs are also contributing to a new pipeline of gene therapies for CVD. Strategies involve the inhibition (e.g. via injection of antagomirs, locked nucleic acid (LNA) anti-miRs, small interfering RNAs (siRNAs) or short hairpin RNAs (shRNAs)) of ncRNAs promoting reparative mechanisms and/or upregulation (e.g. via adeno-associated virus or miRNA precursors) of ncRNAs supporting cardiac regeneration/CM proliferation (99) (Figure 4).

Alternatively, to replenish the myocardium with new cardiomyocytes, direct reprogramming of other cardiac cells into myocytes has been attempted (Figure 4). Due to their frequency and role in scar formation upon injury, fibroblasts are an obvious target. Initial reprogramming strategies were based on the use of cocktails of transcription factors and lately incorporated the use of miRNAs important for muscle development and differentiation (100). In 2013, Nam and colleagues used a combination of four transcription factors (i.e. GATA binding protein 4, Hand2, T-box5 and myocardin) and two miRNAs (i.e. miR-1 and miR-133) to reprogram neonatal and adult human fibroblasts. After 4-11 weeks in culture, fibroblasts revealed transcriptomic alterations, with the silencing of nonmyocytic genes and upregulation of cardiac genes and more importantly, phenotypic changes, exhibiting sarcomere-like structures, calcium transients and contractility (101). Despite recent advances in this area, growing evidence suggests that cardiac fibroblasts, besides producing fibrotic tissue, attain other roles which would be lost upon reprogramming into cardiomyocytes. Furthermore, a recent study combining robust genetic models and cellular markers, reported that cardiac fibroblasts compose a minor population in the murine heart (102). Hence, direct reprogramming of fibroblasts into cardiomyocytes would still have the drawback of not generating enough cardiomyocytes to restore significantly heart function.

In the last decade, considerable attention was given to extracellular vesicles (EVs) and their role in promoting intercellular crosstalk (103). All cardiac subsets release EVs and might interact with target cells in a non-selective or specific way (104). In peripartum cardiomyopathy, upregulation of miR-146a in endothelial cells directs autocrine inhibition of angiogenesis and miR-146a-loaded EVs released by endothelial cells lead to decreased metabolic activity and reduced expression of Erbb4, Notch1 and Irak1 in cardiomyocytes. Indeed, preventing paracrine signalling of miR-146a attenuated peripartum cardiomyopathy (105). This biological process may therefore constitute a mean to modulate cell behaviour. Interestingly, EVs can be loaded with several different components (e.g. ncRNAs, siRNAs, mRNAs and/or proteins). Nevertheless, despite important advances in this field, a better understanding of EV biology is necessary for the development of new therapeutic approaches, including more efficient isolation methods and limiting potential off-targets.

Noteworthy, combination of several of the previous strategies are at present contemplated towards a better therapeutic outcome, yet further investigation is required to understand possible synergistic or antagonistic interactions. 


\section{Thesis Aims and Overview}

The work described in this thesis bridges the fields of cardiovascular research and regenerative medicine. With the aim of further understanding the mechanisms elicited by apex resection in the neonatal murine heart and determine their contribution to cardiac (patho)physiology, we aimed (Aim 1) to elucidate whether the neonatal apex resection injury model constitutes a valuable model to study regenerative mechanisms and to determine the cellular dynamics activated after injury (Aim 2). Furthermore, as most widely used techniques to determine cardiomyocyte proliferation do not distinguish neomyogenesis from binucleation, a process particularly common in the neonatal period, we aimed to develop an optimized protocol, based on stereology and 3D imaging, to estimate cardiomyocyte numbers in the ventricle, thus allowing the discrimination between hyperplasia and hypertrophy (Aim 3).

In addition to the general introduction on cardiac regeneration and novel therapeutic strategies for cardiovascular diseases, this thesis consists of other 4 chapters.

In chapter $\mathbf{2}$ we review the state of the art on how non-coding RNAs act as mediators in endothelial cell signalling and hypoxia during cardiac regeneration. We particularly emphasize on how these small molecules regulate two pivotal processes in cardiac regeneration: cardiomyocyte proliferation and neovascularization.

In chapter $\mathbf{3}$ we provide a thorough characterization of the neonatal heart response to apical resection. A longitudinal assessment of the mechanisms activated over 180 days after surgery, showed that this injury model elicits the co-activation of regenerative and reparative processes. These results are not compatible with full regeneration or repair but rather reflect an intermediate response, endorsing the use of this model in studies focusing on the identification of pro-regenerative and/or anti-fibrotic players.

In chapter 4, we detail a protocol that allows a precise estimation of the number of cardiomyocytes in the heart. This protocol tackles an unmet need in the cardiovascular research as the currently available methodologies are either technically challenging or do not distinguish between cardiomyocyte proliferation and multinucleation. This protocol combines 3D imaging with stereological estimation of the number of cardiomyocytes and thus represents a powerful tool to study regenerative mechanisms or to evaluate the neomyogenic potential of candidate molecules.

In conclusion, chapter 5 summarizes the major findings of the reported experimental data and places this newly generated information in the context of the current state of knowledge in the field of cardiac regenerative medicine. 


\section{References}

1. Naghavi M, Wang H, Lozano R, Davis A, Liang X, Zhou M, Vollset SE, Ozgoren AA, Abdalla S, Abdallah F, Abdel Aziz MI, et al. Global, regional, and national age-sex specific all-cause and cause-specific mortality for 240 causes of death, 1990-2013: a systematic analysis for the Global Burden of Disease Study 2013. Lancet. 2015;385(9963):117-71.

2. Townsend N, Wilson L, Bhatnagar P, Wickramasinghe K, Rayner M, Nichols M. Cardiovascular disease in Europe: epidemiological update 2016. European heart journal. 2016;37(42):3232-45.

3. Yusuf S, Hawken S, Ounpuu S, Dans T, Avezum A, Lanas F, et al. Effect of potentially modifiable risk factors associated with myocardial infarction in 52 countries (the INTERHEART study): case-control study. Lancet (London, England). 2004;364(9438):937-52.

4. John R, Rajasinghe HA, Chen JM, Weinberg AD, Sinha P, Mancini DM, et al. Long-term outcomes after cardiac transplantation: an experience based on different eras of immunosuppressive therapy. The Annals of thoracic surgery. 2001;72(2):440-9.

5. Bergmann O, Bhardwaj RD, Bernard S, Zdunek S, Barnabe-Heider F, Walsh S, et al. Evidence for cardiomyocyte renewal in humans. Science (New York, NY). 2009;324(5923):98-102.

6. Beltrami AP, Urbanek K, Kajstura J, Yan SM, Finato N, Bussani R, et al. Evidence that human cardiac myocytes divide after myocardial infarction. The New England journal of medicine. 2001;344(23):1750-7.

7. Perez-Pomares JM, Gonzalez-Rosa JM, Munoz-Chapuli R. Building the vertebrate heart - an evolutionary approach to cardiac development. Int J Dev Biol. 2009;53(8-10):1427-43.

8. Poss KD, Wilson LG, Keating MT. Heart regeneration in zebrafish. Science (New York, NY). 2002;298(5601):218890.

9. Jopling C, Sleep E, Raya M, Marti M, Raya A, Izpisua Belmonte JC. Zebrafish heart regeneration occurs by cardiomyocyte dedifferentiation and proliferation. Nature. 2010;464(7288):606-9.

10. Puente BN, Kimura W, Muralidhar SA, Moon J, Amatruda JF, Phelps KL, et al. The oxygen-rich postnatal environment induces cardiomyocyte cell-cycle arrest through DNA damage response. Cell. 2014;157(3):565-79.

11. Sander V, Sune G, Jopling C, Morera C, Izpisua Belmonte JC. Isolation and in vitro culture of primary cardiomyocytes from adult zebrafish hearts. Nat Protoc. 2013;8(4):800-9.

12. Paradis AN, Gay MS, Zhang L. Binucleation of cardiomyocytes: the transition from a proliferative to a terminally differentiated state. Drug Discov Today. 2014;19(5):602-9.

13. Porrello ER, Olson EN. A neonatal blueprint for cardiac regeneration. Stem Cell Research. (0).

14. Robledo M. Myocardial regeneration in young rats. The American journal of pathology. 1956;32(6):1215-39.

15. Porrello ER, Mahmoud Al, Simpson E, Hill JA, Richardson JA, Olson EN, et al. Transient Regenerative Potential of the Neonatal Mouse Heart. Science. 2011;331(6020):1078-80.

16. Macmahon HE. Hyperplasia and Regeneration of the Myocardium in Infants and in Children. The American journal of pathology. 1937;13(5):845-54 5.

17. Warthin AS. The Myocardial Lesions of Diphtheria. The Journal of Infectious Diseases. 1924;35(1):32-66.

18. Haubner BJ, Schneider J, Schweigmann U, Schuetz T, Dichtl W, Velik-Salchner C, et al. Functional Recovery of a Human Neonatal Heart After Severe Myocardial Infarction. Circulation research. 2016;118(2):216-21.

19. Saker DM, Walsh-Sukys M, Spector M, Zahka KG. Cardiac recovery and survival after neonatal myocardial infarction. Pediatric cardiology. 1997;18(2):139-42.

20. Andersen DC, Ganesalingam S, Jensen CH, Sheikh SP. Do neonatal mouse hearts regenerate following heart apex resection? Stem cell reports. 2014;2(4):406-13.

21. Andersen DC, Jensen $\mathrm{CH}$, Baun C, Hvidsten S, Zebrowski DC, Engel FB, et al. Persistent scarring and dilated cardiomyopathy suggest incomplete regeneration of the apex resected neonatal mouse myocardium--A 180 days follow up study. Journal of molecular and cellular cardiology. 2016;90:47-52.

22. Andersen DC, Jensen CH, Sheikh SP. Response to Sadek et al. and Kotlikoff et al. Stem cell reports. 2014;3(1):34.

23. Kotlikoff MI, Hesse M, Fleischmann BK. Comment on "Do neonatal mouse hearts regenerate following heart apex resection"? Stem cell reports. 2014;3(1):2.

24. Sadek HA, Martin JF, Takeuchi JK, Leor J, Nie Y, Giacca M, et al. Multi-investigator letter on reproducibility of neonatal heart regeneration following apical resection. Stem cell reports. 2014;3(1):1.

25. Bryant DM, O'Meara CC, Ho NN, Gannon J, Cai L, Lee RT. A systematic analysis of neonatal mouse heart regeneration after apical resection. Journal of molecular and cellular cardiology. 2015;79:315-8.

26. Darehzereshki A, Rubin N, Gamba L, Kim J, Fraser J, Huang Y, et al. Differential regenerative capacity of neonatal mouse hearts after cryoinjury. Developmental biology. 2015;399(1):91-9.

27. Laflamme MA, Murry CE. Heart regeneration. Nature.473(7347):326-35. 
28. Blackburn S. Maternal, Fetal, \& Neonatal Physiology: Elsevier Health Sciences; 2014.

29. Lopaschuk GD, Collins-Nakai RL, Itoi T. Developmental changes in energy substrate use by the heart. Cardiovascular Research. 1992;26(12):1172-80.

30. Nakada Y, Canseco DC, Thet S, Abdisalaam S, Asaithamby A, Santos CX, et al. Hypoxia induces heart regeneration in adult mice. Nature. 2017;541(7636):222-7.

31. Locatelli P, Gimenez CS, Vega MU, Crottogini A, Belaich MN. Targeting the Cardiomyocyte Cell Cycle for Heart Regeneration. Current drug targets. 2019;20(2):241-54.

32. Burton PB, Raff MC, Kerr P, Yacoub MH, Barton PJ. An intrinsic timer that controls cell-cycle withdrawal in cultured cardiac myocytes. Developmental biology. 1999;216(2):659-70.

33. Ikenishi A, Okayama H, Iwamoto N, Yoshitome S, Tane S, Nakamura K, et al. Cell cycle regulation in mouse heart during embryonic and postnatal stages. Development, growth \& differentiation. 2012;54(8):731-8.

34. Tane S, Ikenishi A, Okayama H, Iwamoto N, Nakayama KI, Takeuchi T. CDK inhibitors, p21(Cip1) and p27(Kip1), participate in cell cycle exit of mammalian cardiomyocytes. Biochemical and biophysical research communications. 2014;443(3):1105-9.

35. Mohamed TMA, Ang YS, Radzinsky E, Zhou P, Huang Y, Elfenbein A, et al. Regulation of Cell Cycle to Stimulate Adult Cardiomyocyte Proliferation and Cardiac Regeneration. Cell. 2018;173(1):104-16 e12.

36. Sim CB, Ziemann M, Kaspi A, Harikrishnan KN, Ooi J, Khurana I, et al. Dynamic changes in the cardiac methylome during postnatal development. Faseb j. 2014.

37. Gilsbach R, Preissl S, Gruning BA, Schnick T, Burger L, Benes V, et al. Dynamic DNA methylation orchestrates cardiomyocyte development, maturation and disease. Nat Commun. 2014;5:5288.

38. Dirkx E, da Costa Martins PA, De Windt LJ. Regulation of fetal gene expression in heart failure. Biochim Biophys Acta. 2013;1832(12):2414-24.

39. Liu N, Bezprozvannaya S, Williams AH, Qi X, Richardson JA, Bassel-Duby R, et al. microRNA-133a regulates cardiomyocyte proliferation and suppresses smooth muscle gene expression in the heart. Genes \& development. 2008;22(23):3242-54.

40. Porrello ER, Johnson BA, Aurora AB, Simpson E, Nam YJ, Matkovich SJ, et al. MiR-15 family regulates postnatal mitotic arrest of cardiomyocytes. Circulation research. 2011;109(6):670-9.

41. Porrello ER, Mahmoud AI, Simpson E, Johnson BA, Grinsfelder D, Canseco D, et al. Regulation of neonatal and adult mammalian heart regeneration by the miR-15 family. Proceedings of the National Academy of Sciences of the United States of America. 2013;110(1):187-92.

42. Eulalio A, Mano M, Dal Ferro M, Zentilin L, Sinagra G, Zacchigna S, et al. Functional screening identifies miRNAs inducing cardiac regeneration. Nature. 2012;492(7429):376-81.

43. Gabisonia K, Prosdocimo G, Aquaro GD, Carlucci L, Zentilin L, Secco I, et al. MicroRNA therapy stimulates uncontrolled cardiac repair after myocardial infarction in pigs. Nature. 2019;569(7756):418-22.

44. Pandey R, Yang Y, Jackson L, Ahmed RP. MicroRNAs regulating meis1 expression and inducing cardiomyocyte proliferation. Cardiovascular regenerative medicine. 2016;3.

45. Cai B, Ma W, Ding F, Zhang L, Huang Q, Wang X, et al. The Long Noncoding RNA CAREL Controls Cardiac Regeneration. J Am Coll Cardiol. 2018;72(5):534-50.

46. Epelman S, Lavine Kory J, Beaudin Anna E, Sojka Dorothy K, Carrero Javier A, Calderon B, et al. Embryonic and Adult-Derived Resident Cardiac Macrophages Are Maintained through Distinct Mechanisms at Steady State and during Inflammation. Immunity. 2014;40(1):91-104.

47. Lavine KJ, Epelman S, Uchida K, Weber KJ, Nichols CG, Schilling JD, et al. Distinct macrophage lineages contribute to disparate patterns of cardiac recovery and remodeling in the neonatal and adult heart. Proc Natl Acad Sci U S A. 2014;111(45):16029-34.

48. Howard CM, Baudino TA. Dynamic cell-cell and cell-ECM interactions in the heart. J Mol Cell Cardiol. 2014;70:19-26.

49. leda M, Tsuchihashi T, Ivey KN, Ross RS, Hong TT, Shaw RM, et al. Cardiac fibroblasts regulate myocardial proliferation through beta1 integrin signaling. Developmental cell. 2009;16(2):233-44.

50. Fredj S, Bescond J, Louault C, Delwail A, Lecron JC, Potreau D. Role of interleukin-6 in cardiomyocyte/cardiac fibroblast interactions during myocyte hypertrophy and fibroblast proliferation. J Cell Physiol. 2005;204(2):42836.

51. Ingason AB, Goldstone AB, Paulsen MJ, Thakore AD, Truong VN, Edwards BB, et al. Angiogenesis precedes cardiomyocyte migration in regenerating mammalian hearts. The Journal of thoracic and cardiovascular surgery. 2018;155(3):1118-27 e1.

52. Das S, Goldstone AB, Wang H, Farry J, D'Amato G, Paulsen MJ, et al. A Unique Collateral Artery Development Program Promotes Neonatal Heart Regeneration. Cell. 2019;176(5):1128-42 e18.

53. Mahmoud Al, O'Meara CC, Gemberling M, Zhao L, Bryant DM, Zheng R, et al. Nerves Regulate Cardiomyocyte Proliferation and Heart Regeneration. Developmental cell. 2015;34(4):387-99. 
54. Goldsmith EC, Hoffman A, Morales MO, Potts JD, Price RL, McFadden A, et al. Organization of fibroblasts in the heart. Dev Dyn. 2004;230(4):787-94.

55. Hanson KP, Jung JP, Tran QA, Hsu SP, lida R, Ajeti V, et al. Spatial and Temporal Analysis of Extracellular Matrix Proteins in the Developing Murine Heart: A Blueprint for Regeneration. Tissue Eng Part A. 2013.

56. Hurle JM, Icardo JM, Ojeda JL. Compositional and structural heterogenicity of the cardiac jelly of the chick embryo tubular heart: a TEM, SEM and histochemical study. J Embryol Exp Morphol. 1980;56:211-23.

57. Kim H, Yoon CS, Kim H, Rah B. Expression of extracellular matrix components fibronectin and laminin in the human fetal heart. Cell Struct Funct. 1999;24(1):19-26.

58. Barry A. The functional significance of the cardiac jelly in the tubular heart of the chick embryo. Anat Rec. 1948;102(3):289-98.

59. Lockhart M, Wirrig E, Phelps A, Wessels A. Extracellular matrix and heart development. Birth Defects Res A Clin Mol Teratol. 2011;91(6):535-50.

60. Gazoti Debessa CR, Mesiano Maifrino LB, Rodrigues de Souza R. Age related changes of the collagen network of the human heart. Mech Ageing Dev. 2001;122(10):1049-58.

61. Williams C, Quinn KP, Georgakoudi I, Black LD, 3rd. Young developmental age cardiac extracellular matrix promotes the expansion of neonatal cardiomyocytes in vitro. Acta Biomater. 2014;10(1):194-204.

62. Bowers SL, Banerjee I, Baudino TA. The extracellular matrix: at the center of it all. J Mol Cell Cardiol. 2010;48(3):474-82.

63. Camelliti P, Borg TK, Kohl P. Structural and functional characterisation of cardiac fibroblasts. Cardiovascular Research. 2005;65(1):40-51.

64. Silva AC, Rodrigues SC, Caldeira J, Nunes AM, Sampaio-Pinto V, Resende TP, et al. Three-dimensional scaffolds of fetal decellularized hearts exhibit enhanced potential to support cardiac cells in comparison to the adult. Biomaterials. 2016;104:52-64.

65. Menasche P, Alfieri O, Janssens S, McKenna W, Reichenspurner H, Trinquart L, et al. The Myoblast Autologous Grafting in Ischemic Cardiomyopathy (MAGIC) trial: first randomized placebo-controlled study of myoblast transplantation. Circulation. 2008;117(9):1189-200.

66. Gnecchi M, Zhang Z, Ni A, Dzau VJ. Paracrine mechanisms in adult stem cell signaling and therapy. Circulation research. 2008;103(11):1204-19.

67. Murry CE, Soonpaa MH, Reinecke H, Nakajima H, Nakajima HO, Rubart M, et al. Haematopoietic stem cells do not transdifferentiate into cardiac myocytes in myocardial infarcts. Nature. 2004;428(6983):664-8.

68. Orlic D, Kajstura J, Chimenti S, Jakoniuk I, Anderson SM, Li B, et al. Bone marrow cells regenerate infarcted myocardium. Nature. 2001;410(6829):701-5.

69. Traverse JH, Henry TD, Pepine CJ, Willerson JT, Zhao DX, Ellis SG, et al. Effect of the use and timing of bone marrow mononuclear cell delivery on left ventricular function after acute myocardial infarction: the TIME randomized trial. Jama. 2012;308(22):2380-9.

70. Musialek P, Mazurek A, Jarocha D, Tekieli L, Szot W, Kostkiewicz M, et al. Myocardial regeneration strategy using Wharton's jelly mesenchymal stem cells as an off-the-shelf 'unlimited' therapeutic agent: results from the Acute Myocardial Infarction First-in-Man Study. Postepy w kardiologii interwencyjnej = Advances in interventional cardiology. 2015;11(2):100-7.

71. Santos Nascimento D, Mosqueira D, Sousa LM, Teixeira M, Filipe M, Resende TP, et al. Human umbilical cord tissue-derived mesenchymal stromal cells attenuate remodeling after myocardial infarction by proangiogenic, antiapoptotic, and endogenous cell-activation mechanisms. Stem cell research \& therapy. 2014;5(1):5.

72. Mathiasen AB, Qayyum AA, Jorgensen E, Helqvist S, Fischer-Nielsen A, Kofoed KF, et al. Bone marrow-derived mesenchymal stromal cell treatment in patients with severe ischaemic heart failure: a randomized placebocontrolled trial (MSC-HF trial). European heart journal. 2015;36(27):1744-53.

73. Uchida S, De Gaspari P, Kostin S, Jenniches K, Kilic A, Izumiya Y, et al. Sca1-derived cells are a source of myocardial renewal in the murine adult heart. Stem cell reports. 2013;1(5):397-410.

74. Valente M, Nascimento DS, Cumano A, Pinto-do OP. Sca-1+ cardiac progenitor cells and heart-making: a critical synopsis. Stem cells and development. 2014;23(19):2263-73.

75. Li Y, He L, Huang X, Bhaloo SI, Zhao H, Zhang S, et al. Genetic Lineage Tracing of Nonmyocyte Population by Dual Recombinases. Circulation. 2018;138(8):793-805.

76. Sultana N, Zhang L, Yan J, Chen J, Cai W, Razzaque S, et al. Resident c-kit(+) cells in the heart are not cardiac stem cells. Nature communications. 2015;6:8701.

77. van Berlo JH, Kanisicak O, Maillet M, Vagnozzi RJ, Karch J, Lin SC, et al. c-kit+ cells minimally contribute cardiomyocytes to the heart. Nature. 2014;509(7500):337-41.

78. Bolli R, Chugh AR, D'Amario D, Loughran JH, Stoddard MF, Ikram S, et al. Cardiac stem cells in patients with ischaemic cardiomyopathy (SCIPIO): initial results of a randomised phase 1 trial. Lancet (London, England). 2011;378(9806):1847-57. 
79. Makkar RR, Smith RR, Cheng K, Malliaras K, Thomson LE, Berman D, et al. Intracoronary cardiosphere-derived cells for heart regeneration after myocardial infarction (CADUCEUS): a prospective, randomised phase 1 trial. Lancet (London, England). 2012;379(9819):895-904.

80. Beltrami AP, Barlucchi L, Torella D, Baker M, Limana F, Chimenti S, et al. Adult cardiac stem cells are multipotent and support myocardial regeneration. Cell. 2003;114(6):763-76.

81. Dyer O. NEJM retracts article from former researcher once hailed as heart stem cell pioneer. BMJ. 2018;363:k4432.

82. The Lancet E. Expression of concern: the SCIPIO trial. Lancet (London, England). 2014;383(9925):1279.

83. Lundy SD, Gantz JA, Pagan CM, Filice D, Laflamme MA. Pluripotent stem cell derived cardiomyocytes for cardiac repair. Current treatment options in cardiovascular medicine. 2014;16(7):319.

84. Lundy SD, Zhu WZ, Regnier M, Laflamme MA. Structural and functional maturation of cardiomyocytes derived from human pluripotent stem cells. Stem cells and development. 2013;22(14):1991-2002.

85. Madonna R, Van Laake L, Davidson SM, Engel FB, Hausenloy DJ, Lecour S, et al. Position Paper of the European Society of Cardiology Working Group Cellular Biology of the Heart: cell-based therapies for myocardial repair and regeneration in ischemic heart disease and heart failure. European heart journal. 2016;37(23):1789-98d.

86. Tu C, Chao BS, Wu JC. Strategies for Improving the Maturity of Human Induced Pluripotent Stem Cell-Derived Cardiomyocytes. Circulation research. 2018;123(5):512-4.

87. Nicks Amy M, Humphreys David T, Holman Sara R, Chan Andrea Y, Djordjevic D, Naqvi N, et al. Abstract 15573 : Transcription Factors Driving Postnatal Cardiomyocyte Maturation. Circulation. 2018;138(Suppl_1):A15573-A.

88. Chong JJ, Yang X, Don CW, Minami E, Liu YW, Weyers JJ, et al. Human embryonic-stem-cell-derived cardiomyocytes regenerate non-human primate hearts. Nature. 2014;510(7504):273-7.

89. Menasche P, Vanneaux V, Hagege A, Bel A, Cholley B, Cacciapuoti I, et al. Human embryonic stem cell-derived cardiac progenitors for severe heart failure treatment: first clinical case report. European heart journal. 2015;36(30):2011-7.

90. Bui QT, Gertz ZM, Wilensky RL. Intracoronary delivery of bone-marrow-derived stem cells. Stem cell research \& therapy. 2010;1(4):29.

91. Muller-Ehmsen J, Krausgrill B, Burst V, Schenk K, Neisen UC, Fries JW, et al. Effective engraftment but poor mid-term persistence of mononuclear and mesenchymal bone marrow cells in acute and chronic rat myocardial infarction. Journal of molecular and cellular cardiology. 2006;41(5):876-84.

92. Cambria E, Pasqualini FS, Wolint P, Gunter J, Steiger J, Bopp A, et al. Translational cardiac stem cell therapy: advancing from first-generation to next-generation cell types. NPJ Regenerative medicine. 2017;2(1):17.

93. Song $\mathrm{Y}$, Zhang $\mathrm{C}$, Zhang J, Sun N, Huang K, Li H, et al. An injectable silk sericin hydrogel promotes cardiac functional recovery after ischemic myocardial infarction. Acta biomaterialia. 2016;41:210-23.

94. Georgiadis V, Knight RA, Jayasinghe SN, Stephanou A. Cardiac tissue engineering: renewing the arsenal for the battle against heart disease. Integrative biology : quantitative biosciences from nano to macro. 2014;6(2):111-26. 95. Gouveia PJ, Rosa S, Ricotti L, Abecasis B, Almeida HV, Monteiro L, et al. Flexible nanofilms coated with aligned piezoelectric microfibers preserve the contractility of cardiomyocytes. Biomaterials. 2017;139:213-28.

96. Stewart DJ, Kutryk MJ, Fitchett D, Freeman M, Camack N, Su Y, et al. VEGF gene therapy fails to improve perfusion of ischemic myocardium in patients with advanced coronary disease: results of the NORTHERN trial. Molecular therapy : the journal of the American Society of Gene Therapy. 2009;17(6):1109-15.

97. Ripa RS, Jorgensen E, Wang Y, Thune JJ, Nilsson JC, Sondergaard L, et al. Stem cell mobilization induced by subcutaneous granulocyte-colony stimulating factor to improve cardiac regeneration after acute ST-elevation myocardial infarction: result of the double-blind, randomized, placebo-controlled stem cells in myocardial infarction (STEMMI) trial. Circulation. 2006;113(16):1983-92.

98. Ott I, Schulz S, Mehilli J, Fichtner S, Hadamitzky M, Hoppe K, et al. Erythropoietin in patients with acute STsegment elevation myocardial infarction undergoing primary percutaneous coronary intervention: a randomized, double-blind trial. Circulation Cardiovascular interventions. 2010;3(5):408-13.

99. Lucas T, Bonauer A, Dimmeler S. RNA Therapeutics in Cardiovascular Disease. Circulation research. 2018;123(2):205-20.

100. Raso A, Dirkx E. Cardiac regenerative medicine: At the crossroad of microRNA function and biotechnology. Noncoding RNA Res. 2017;2(1):27-37.

101. Nam YJ, Song K, Luo X, Daniel E, Lambeth K, West K, et al. Reprogramming of human fibroblasts toward a cardiac fate. Proceedings of the National Academy of Sciences of the United States of America. 2013;110(14):558893.

102. Pinto AR, Ilinykh A, Ivey MJ, Kuwabara JT, D'Antoni ML, Debuque R, et al. Revisiting Cardiac Cellular Composition. Circulation research. 2016;118(3):400-9.

103. Ottaviani L, Sansonetti M, da Costa Martins PA. Myocardial cell-to-cell communication via microRNAs. Noncoding RNA Res. 2018;3(3):144-53. 
104. Corrado C, Raimondo S, Chiesi A, Ciccia F, De Leo G, Alessandro R. Exosomes as intercellular signaling organelles involved in health and disease: basic science and clinical applications. International journal of molecular sciences. 2013;14(3):5338-66.

105. Halkein J, Tabruyn SP, Ricke-Hoch M, Haghikia A, Nguyen NQ, Scherr M, et al. MicroRNA-146a is a therapeutic target and biomarker for peripartum cardiomyopathy. The Journal of clinical investigation. 2013;123(5):2143-54. 


\section{CHAPTER 2}

\section{Non-coding RNAs in endothelial cell signaling and hypoxia during cardiac regeneration}

Marijn M. C. Peters ${ }^{a}$, Vasco Sampaio-Pinto ${ }^{b, c, d, e, f}$, Paula A. da Costa Martins ${ }^{e, f, g}$

a Department of Cardiology, Laboratory of Experimental Cardiology, UMC Utrecht Regenerative Medicine Centre, University Medical Centre Utrecht, University Utrecht, Utrecht, The Netherlands;

${ }^{b}$ i3S - Instituto de Investigação e Inovação em Saúde, Universidade do Porto, Porto, Portugal;

c INEB - Instituto Nacional de Engenharia Biomédica, Universidade do Porto, Porto, Portugal;

d ICBAS - Instituto de Ciências Biomédicas Abel Salazar, Universidade do Porto, Portugal;

e CARIM School for Cardiovascular Diseases, Faculty of Health, Medicine and Life Sciences, Maastricht University, Maastricht, The Netherlands;

${ }^{f}$ iMAiA - Institute of Molecular Biology and RNA Technology, Faculty of Sciences and Engineering, Maastricht University, Maastricht, The Netherlands;

${ }^{g}$ Department of Physiology and Cardiothoracic Surgery, Faculty of Medicine, University of Porto, Porto, Portugal.

Biochim Biophys Acta Mol Cell Res. 2019 Jul 27. pii: S0167-4889(18)30513-5

doi: 10.1016/j.bbamcr.2019.07.010 


\begin{abstract}
Heart failure (HF) as a result of myocardial infarction (MI) is the leading cause of death worldwide. In contrast to the adult mammalian heart, which has low regenerative capacity, newborn mammalian and zebrafish hearts can completely regenerate after injury. Cardiac regeneration is considered to be mediated by proliferation of pre-existing cardiomyocytes (CMs) mainly located in a hypoxic niche. To find new therapies to treat HF, efforts are being made to understand the molecular pathways underlying the regenerative capacity of the heart. However, the multicellularity of the heart is important during cardiac regeneration as not only $\mathrm{CM}$ proliferation but also the restoration of the endothelium is imperative to prevent progression to HF. It has recently come to light that signalling from non-coding RNAs (ncRNAs) and extracellular vesicles (EVs) plays a role in the healthy and the diseased heart. Multiple studies identified differentially expressed non-coding RNAs after MI, making them potential therapeutic targets. In this review, we highlight the molecular interactions between endothelial cells (ECs) and CMs in cardiac regeneration and when the heart loses its regenerative capacity. We specifically emphasize the role of ncRNAs and cell-cell communication via EVs during cardiac regeneration and neovascularisation.
\end{abstract}




\section{Introduction}

The non-coding part of the genome has come to light as an essential regulator in physiology and disease [1]. Non-coding genes can be subdivided into different categories based on their length: short non-coding genes ( $<200$ nucleotides (nt)) and long non-coding genes (>200 nt) [2]. Within small non-coding RNAs (ncRNAs), microRNAs (miRNAs/miRs) received increasing attention as mediators of mRNA expression in multiple diseases. Less understood classes of ncRNAs including transcribed ultraconserved regions (T-UCRs), small nucleolar RNAs (snoRNAs), PIWI-interacting RNAs (piRNAs), large intergenic non-coding RNAs (lincRNAs) and long non-coding RNAs (IncRNAs) are also being associated with the development of human diseases [1]. Among other functions, ncRNAs are known for the inhibition of mRNAs, transposon repression, DNA methylation, chromatin modification and influencing mRNA stability. High conservation of non-coding regions across phylogeny denotates an important role of ncRNAs in regulating gene expression [3]. Single-nucleotide polymorphisms (SNPs) in non-coding regions or disruption of ncRNA expression have been associated with the progression of cardiovascular diseases [4]. Furthermore, paracrine signalling of ncRNAs via extracellular vesicles (EVs) has recently received increasing attention as a molecular mechanism influencing pathophysiology and as a potential therapeutic target for cardiovascular diseases $[5,6]$.

The human heart has limited regenerative capacity. As such, in response to myocardial infarction (MI), the injured myocardium is replaced by a fibrotic scar. Extensive remodelling of the myocardium after injury can limit the contractile capacity of the heart and lead to heart failure (HF). At steady state, cardiomyocyte (CM) renewal replaces apoptotic $\mathrm{CMs}$ at a rate of $0,5-1 \%$ per year [7]. Even though CM proliferation rate increases after injury, it remains insufficient to fully regenerate the lost myocardial tissue [8]. In contrast to the adult mammalian heart, neonatal mammal and zebrafish hearts display efficient myocardial regeneration after injury [9-11]. Evidence supports that the time window for mammalian heart regeneration is defined by a postnatal shift towards aerobic metabolism which promotes CM cell cycle arrest [12]. Hypoxia signalling is a key mechanism for cardiac regeneration in the zebrafish heart and is achieved by the absence of endothelial cells (ECS) adjacent to $\mathrm{CMs}[13,14]$. The main strategy aiming to improve the regenerative capacity of the heart has been to stimulate CM proliferation [15]. However, it is important to note that the heart is not solely composed of CMs and that myocardial function also depends on other cell types and their molecular interactions. The healthy human heart consists of an estimated 2-3 billion CMs, making up to a third of the total number of cells and $70 \%$ of the cardiac volume [16] whereas mesenchymal cells (e.g. cardiac fibroblasts (CFs)) were found to account to half of cardiac cells in the human heart [17]. The contribution of ECs and smooth muscle cells (SMCs) to the cardiac cellular composition has long been considered minor. However, a recent study combining robust genetic models and cellular markers showed that the non-myocyte population of the murine heart consists of $>60 \%$ ECs and $<20 \%$ CFs [18]. This revisited cardiac cell composition suggests that ECs are present in a much higher frequency to what was classically predicted, which might have direct 
implications on cardiac pathophysiology [19]. Hence, inducing only CM proliferation after $\mathrm{MI}$ may lead to defective heart regeneration as newly formed CMs are unlikely to survive the post-ischaemic microenvironment in the absence of oxygen-supplying ECs [20].

$\mathrm{CMs}$ interact with surrounding cells via physical contact and paracrine signalling. Upon $\mathrm{MI}$, CMs in the infarcted area undergo ischaemic cell death and apoptosis [21]. CMs disassemble their sarcomeres and mitochondria, condense their chromatin and lose their rod-shaped morphology [22]. The interaction between apoptotic CMs and the microvascular network of ECs post-MI has been studied extensively [23]. Both hypoxia and reperfusion disrupt the redox states in CMs leading to oxidative stress, impaired mitochondrial oxidative phosphorylation and eventually to apoptosis during the acute phase after $\mathrm{MI}$, in a process mediated by activation of caspase-3 [23]. Unexpectedly, it has been suggested that $\mathrm{CMs}$ with potential to re-enter the cell cycle proliferate due to extracellular signals present in the infarct border-zone [24], which correlates with a reduction in capillary density and decreased signalling from ECs. Hypoxia inducible factor $1 \alpha(H i f 1 \alpha)$ stabilization in CMs located in a hypoxic microenvironment was found to be a hallmark of CM proliferation [25]. Hif1-regulated genes are involved in $\mathrm{O}_{2}$ delivery and consumption, glycolysis, mitochondrial function, cell survival, antioxidant defence, and angiogenesis [26]. In fact, loss of hypoxia signalling prevented effective regeneration of the zebrafish heart [14] and the small subset of cycling CMs in the adult mammalian heart were located in a hypoxic niche [25]. Hif1 $\alpha$ is the main regulator of the hypoxia-induced stress response as its activity is regulated by post-translational ubiquitination and degradation under normoxic conditions $[25,27]$. Under low environmental oxygen, hydroxylation and subsequent ubiquitination of Hif $1 \alpha$ do not take place allowing its translocation to the nucleus and activation of gene expression.

Postnatal CMs lose their ability to proliferate when their metabolism changes from anaerobic glycolysis to oxidative phosphorylation [12]. ROS, oxidative DNA damage, and DNA damage response (DDR) markers significantly increase during the postnatal period, which correlates with a progressive loss of cardiac regeneration. Inversely, stimulation of postnatal hypoxemia increased the proliferative time window of CMs [12]. The important role of low environmental oxygen and Hif1 $\alpha$ stabilization in $\mathrm{CM}$ proliferation contradicts evidence that fast revascularisation is essential to support cardiac regeneration [28]. Yet, the neonatal murine heart is heavily vascularised after regeneration $[9,11]$ and without vascularisation of the injured area, regeneration ends prematurely [29, 30]. Taken together, CM proliferation, hypoxia and neovascularization seem to be pivotal processes for cardiac regeneration that exhibit a very precise spatiotemporal regulation (Fig. 1). Understanding the intrinsic molecular mechanisms underlying the role of hypoxia and vascularisation in CM proliferation may uncover the importance of EC-CM signalling in cardiac regeneration. Increasing evidence indicates that the molecular mechanisms involved in the heart response after $\mathrm{Ml}$ and cardiac regeneration are also under epigenetic control of ncRNAs (Fig. 1). 


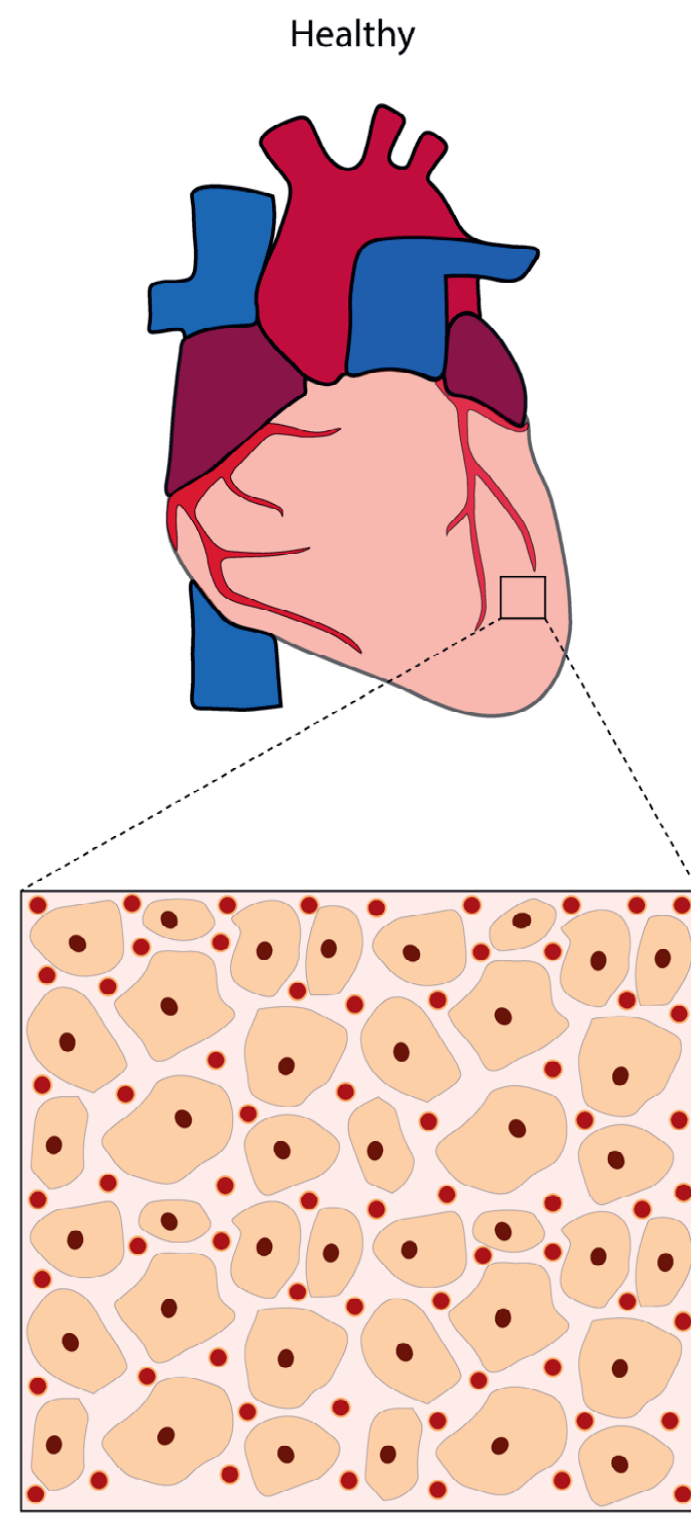

EC:CM Ratio (4:1)

CM Survival
Myocardial Infarction

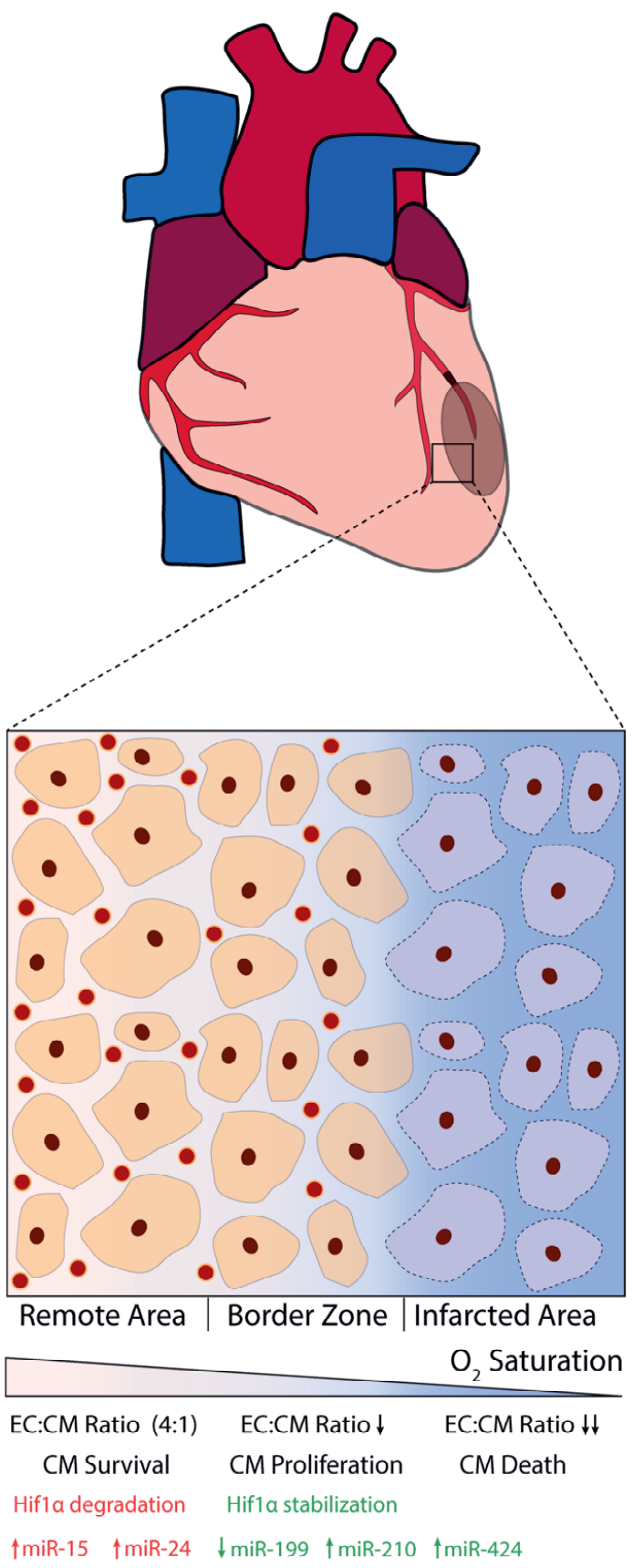

Fig.1 - Hypoxia Signalling is Essential for Cardiac Regeneration

Following an ischemic event, the myocardium is marked by extensive cell death (infarcted area). In the border zone, lower EC:CM ratio activates a hypoxic signalling cascade (e.g. Hif1 $\alpha$ stabilization) characterized by induction of EC and CM proliferation. Subsequent revascularization of the border-zone increases oxygen saturation, reducing hypoxia signalling (e.g. Hif1 $\alpha$ degradation) and limiting CM proliferation. Higher oxygen levels, also found in the remote myocardium, favour CM survival. Neovascularization and neomyogenesis are tightly regulated processes and modulated by ncRNAs. In response to hypoxia, miR-199 (downregulated in CMs), miR-210 (upregulated in CMs, ECs and SMCs) and miR424 (upregulated in ECs) favour Hif1 $\alpha$ stabilization. In contrast, miR-15a,b (upregulated in CMs) and miR-24 (upregulated in ECs) favour Hif1 $\alpha$ degradation. 


\section{Non-coding RNAs in endothelial cell signalling}

ncRNAs are well established mediators of EC proliferation and mediate the interaction between ECs and CMs as paracrine elements. Differential expression of ncRNAs in ECs and CMs after ischaemic injury indicates the potential of ncRNAs as regulators of regeneration and neovascularisation. IncRNAs and miRNAs have also been shown to be regulated by hypoxia [31-33] and have been proposed as potential therapeutic strategies to modulate tissue vascularisation [34]. Hence, stimulation or inhibition of ncRNA expression may be used as a therapeutic tool to induce cardiac regeneration.

\section{miRNAs}

miRNAs (miRs) are single stranded, small ncRNA molecules ( 22 nucleotides) encoded in intergenic regions of the genome that inhibit gene expression by binding to the three prime untranslated region ( $3^{\prime} \mathrm{UTR}$ ) of complementary target mRNAs [4]. Binding can block the translation of the target mRNA or cause mRNA degradation depending on the degree of complementarity between the miRNA and the target mRNA. miRNAs have been found to control many cellular processes including cell survival, differentiation and proliferation [35]. Furthermore, alterations in cardiac miRNA pathways can induce cardiomyopathies and HF $[36,37]$. The discovery that miRNAs are also important in angiogenesis came from a genetic knockout of the endoRNAse Dicer, an essential protein processing pre-miRNAs into mature miRNAs, as these animals displayed severely impaired angiogenesis [38, 39]. Multiple miRNAs have been found to play a pivotal role in regulating angiogenesis by impairing or inducing EC proliferation $[2,35,40]$. miRNAs that influence angiogenesis can influence the expression of coding genes in angiogenic signalling pathways (e.g. multiple miRNAs expressed during hypoxia were found to regulate vascular endothelial growth factor (Vegf) expression [41]) (Table 1). Inversely, the expression of miRNAs can be influenced by coding genes involved in angiogenesis. VEGF has been shown to induce the expression of miR-20a and miR-31, two miRNAs that can bind the 3'UTR of a negative regulator of angiogenesis, tumour necrosis factor superfamily-15 (TNFSF15) [42]. Furthermore, hypoxia was also shown to affect miRNA-mediated signalling between ECs and CMs [40, 43]. miRNAs that are involved in the hypoxia signalling were recently named hypoxamiRs and were identified as crucial components to regulate cellular and molecular responses to decreased oxygen tension [40, 44]. HIF1 $\alpha$ is modulated by several miRNAs in ischaemic heart disease $[44,45]$ and prevents hypoxia-induced mitochondrial damage [33]. Indeed, downregulation of hypoxamiR-199 in ischaemic CMs led to the stabilization and upregulation of hif1 $\alpha$ expression [33]. This occurs through de-repression of SIRTUIN-1, a class III histone deacetylase targeted by hypoxamiR-199 which, in turn, prevents PHD2-mediated destabilization of HIF1 $\alpha$ [33]. PHD2 downregulation by SIRTUIN-1 is mediated by NADdependent deacetylase activity of SIRTUIN-1. Sirtuin-1 is also targeted by the endothelial expressed miR-24, a miRNA involved in endothelial cell apoptosis through targeting of the endothelium-enriched transcription factor GATA2 and the p21-activated kinase PAK4 [31]. 
Silencing of endothelial miR-24 in mouse hearts after Ml limited the infarct size and increased cardiac function.

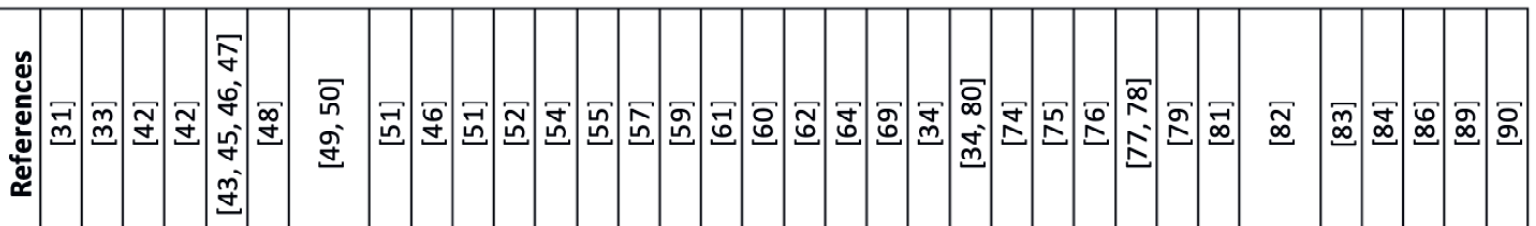

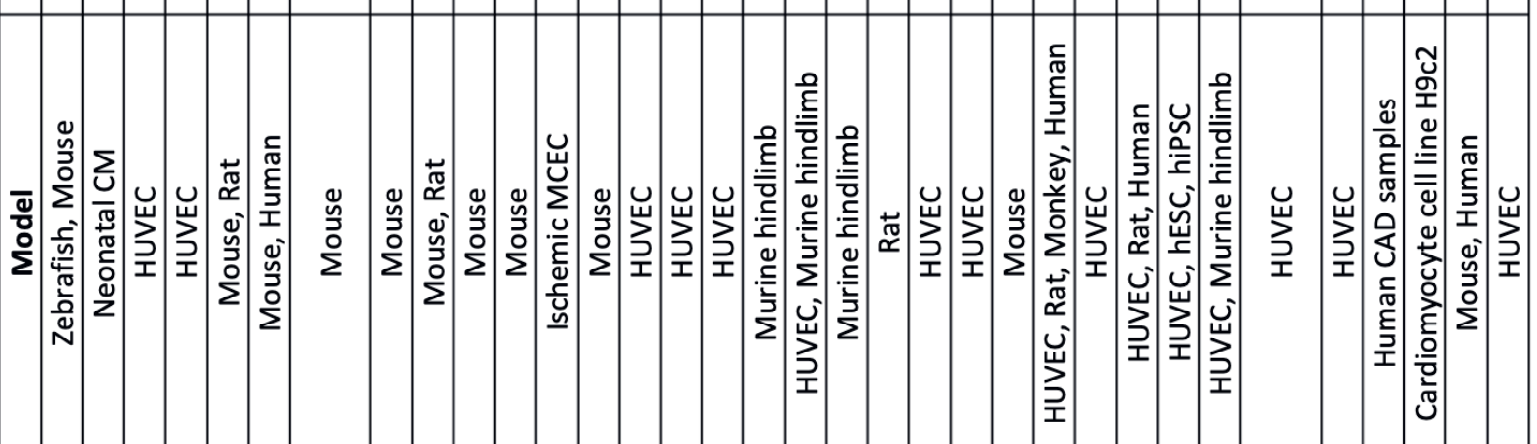

竞

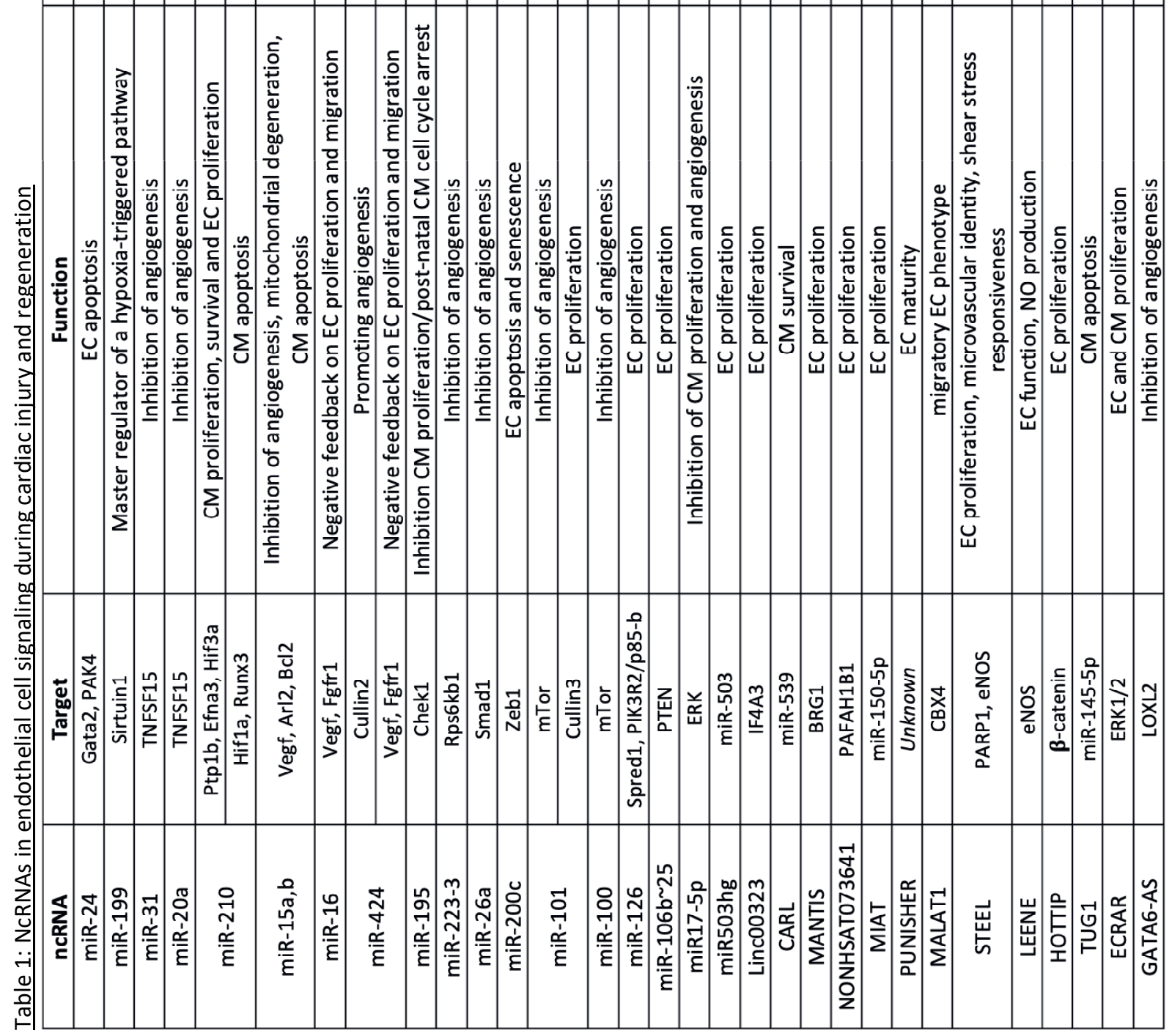


miR-210, another hypoxamiR, is ubiquitously upregulated in multiple cardiac cell types during ischaemic injury including ECs, SMCs and CMs [46]. During hif1 $\alpha$ stabilization, miR210 expression is increased in CMs repressing its targets protein tyrosine phosphatase $1 b$ (Ptp1b), ephrin A3 (Efna3) and Hif3a. Intramyocardial injections of miR-210 increased CM proliferation and survival and endothelial cell proliferation [43, 45, 47]. However, another study found detrimental effects of miR-210 in mouse hearts through repression of its target hif1 $\alpha$ in ECs and CMs [48]. The authors also suggest that miR-210 might play a different role in the mouse and human ischaemic myocardium. The exact molecular mechanisms of miR210 signalling in the post-ischaemic human heart remain to be investigated.

Members of the miR-15 family (miR-195, miR-15b, miR-16-1, miR-16-2, miR-424, and miR$497)$ are also increased during hypoxia $[40,49]$. miR-15b inhibits the translation of Vegf, therefore limiting neovascularisation of the ischaemic tissue. In addition, ADP ribosylation factor-like 2 ( $A r / 2$ ) targeting by miR-15 has been reported to be involved in mitochondrial degeneration and resulting cardiac dysfunction [49]. Moreover, miR-15 targets B-cell lymphoma 2 (BCl-2) mRNA promoting CM apoptosis during hypoxia [50]. miR-16 and miR424 target Vegf and fibroblast growth factor receptor 1 (Fgfr1) reducing proliferation and migration of ECs [51]. In contrast, hypoxia increased expression of miRNA-424 in cardiac ECs was reported to promote angiogenesis by repressing Cullin2 [46]. Cullin2 is a scaffolding protein required to assemble the ubiquitin ligase system involved in the degradation of HIF1A and inhibition of Cullin2 led to HIF1A stabilization [46]. Differential reported effects of the miR-424 might be caused by different transfection methods and efficiencies or cell autonomous/non-autonomous documented effects $[41,51]$. Furthermore, cardiac specific mechanisms might explain the reported diverging effects as cardiac ECs can have a different phenotype and response to miRNA signalling.

Recent studies have demonstrated the role of the miR-15 family in regulating the postnatal CM cell cycle arrest [52]. Transfecting mouse hearts with antimiR-195 inhibited the targetbinding function of miR-195 and improved cardiac function by stimulating CM proliferation [53]. From this perspective, the miR-15 family, in preventing both CM proliferation and angiogenesis, serves as a promising target for regenerative therapy.

Endogenous endothelial miRNAs (e.g. miR-26a, miR-24, miR-34c, miR-375, miR-223) are upregulated in the infarct area after $\mathrm{Ml}$ and inhibit angiogenesis [31, 54-56]. Furthermore, miRNA-200c is upregulated in ischaemic ECs and induces endothelial cell apoptosis by targeting Zeb1 [57]. In contrast, some miRNAs promote coronary circulation and cardiac microcirculation [58]. Other pro-angiogenic hypoxamiRs like miRNA-101 and miRNA-100 express their functional properties by targeting Heme oxygenase- 1 and Cullin-3 signalling and Rapamycin, respectively [59-61]. miR-126 has been found to be highly expressed in healthy ECs and decreased levels could predict impaired coronary vascularisation and coronary collaterals [62]. miR-126 is especially enriched in mouse embryos and regulates the response of ECS to VEGF through targeting of Sprouty-related protein (SPRED1) and phosphoinositol-3-kinase regulatory sub-unit 2 (PIK3R2/p85-b) [63]. Likewise, the miR$106 b^{\sim} 25$ cluster has been found to be essential for EC proliferation in hindlimb ischemia in mice [64]. Similar mechanisms are likely to play a role in cardiac revascularisation. 
miRNAs expressed by CMs can also contribute to the secretion of specific factors that influence endothelial cell behaviour and angiogenesis [65] or be transported themselves via exosomes to ECs and regulate angiogenesis $[66,67]$. This allows for tight regulation of neovascularisation in the adult heart and provides multiple entry-points for possible therapeutic targets to stimulate cardiac regeneration. A recent study performed a genomewide profiling of ncRNAs in the developing and maturing heart and identified differentially expressed miRNAs that could underlie the change in regenerative capacity including miR17-5p, miR-122-5p and miR-20a-5p [68]. Additionally, miR-17-5p was found to suppress the formation of blood vessels [69]. Furthermore, RNA sequencing data of murine hearts during early stages of postnatal life, at which the regenerative capacity of the heart is gradually lost, showed a marked change in expression of miRNAs both in ECs and CMs between P3 and P5 [70].

\section{LncRNAs}

LncRNAs (>200 nt) have been found to contribute to biological processes through regulating epigenetic gene silencing or by functioning as a protein scaffold [2]. Most IncRNAs show low sequence conservation between species and most have arisen only within the primate lineage [71]. Interactions between IncRNAs and miRNAs also play a role in vascularisation [72]. LncRNAs can bind to the nucleotide sequence targeted by miRNAs, circumventing their effect on mRNA targets [73]. In addition, miRNAs can bind IncRNAs and influence IncRNA stability and induce miRNA-mediated decay [72]. Differential regulation of IncRNAs in heart development, disease and also during regeneration and repair provided an indication of the potential to target IncRNAs therapeutically (Table 1). Cardiac apoptosis-related IncRNA (CARL) expression was found to be increased in hypoxic CMs. Furthermore, sequestering of miR-539 by CARL IncRNA prevented mitochondrial fission and apoptosis by downregulating the miR-539 mediated inhibition of PHB2 in a mouse model of ischemia/reperfusion injury [74]. Hypoxia-sensitive LncRNA miR503hg is located next to the encoding sequence of miR424 and is involved in the pro-angiogenic response during hypoxia through cis-regulatory action on miR-424 [34].

The pro-angiogenic property of ECs was recently described to be facilitated by the expression of the IncRNA MANTIS [75] and NONHSAT073641 [76]. Furthermore, analysis of the expression of IncRNAs in the heart identified myocardial infarction-associated transcript (MIAT) and PUNISHER to be connected to changes in cardiac microvasculature. The presence of IncRNA MIAT after a MI suggested a role of IncRNAs in the post-ischaemic heart [77] and further investigation identified its role in regulating endothelial cell function and pathological angiogenesis [78]. Through interaction with endogenous endothelial miR-150$5 p$, which represses Vegf translation, MIAT causes an abnormal upregulation of VEGF. The aberrant expression of VEGF leads to pathological angiogenesis and microvascular dysfunction [78]. PUNISHER acts in the development of the vasculature and is expressed in mature ECS [79]. Inhibition of PUNISHER is associated with severe defects in vascular branching and vessel formation [79]. Furthermore, other IncRNAs have been described to 
modulate endothelial cell proliferation and might also be involved in regulating cardiac angiogenesis. LncRNA Linc00323 has been reported to bind IF4A3 to mediate GATA2 expression maintaining vascular structural integrity and endothelial cell proliferation [34, 80]. Moreover, pro-angiogenic IncRNA metastasis-associated lung adenocarcinoma transcript 1 (MALAT1) is upregulated during hypoxia in ECs and is involved in the balance between a proliferative and a migratory EC phenotype [81].

Recently, spliced-transcript endothelial enriched IncRNA (STEEL) was discovered as an important IncRNA involved in the EC angiogenic potential, microvascular identity, and shear stress responsiveness [82]. Furthermore, enhancer-associated IncRNA (LEENE) was identified as a regulator in eNOS signalling and EC function [83]. HOTTIP expression was increased in ECs in coronary artery disease tissue and induced EC proliferation via Wnt/ $\beta$ catenin signalling [84]. Mitochondrial derived IncRNA LIPCAR was identified as a biomarker of cardiac remodelling with increased expression during post-MI remodelling and chronic $\mathrm{HF}$ and its expression has been suggested to be related to changes in oxidative phosphorylation in CMs [85]. However, much remains unknown on the role of IncRNAs in regulating cardiac angiogenesis and regeneration after $\mathrm{MI}$.

The communication between CMs and ECs can also be mediated by IncRNAs and can potentially influence neovascularisation and regeneration (Table 1). LncRNA TUG1 expression increased during hypoxia and promoted cell apoptosis by regulating the miR145-5p-Bnip3 axis [86]. miR-145 exerts a protective effect against oxidative stress in CMs by regulating Bnip3 expression, a mitochondrial sensory of oxidative stress. The IncRNA AZIN2sv was identified in the rat heart to inhibit CM proliferation and loss of AZIN2sv expression promoted cardiac regeneration and attenuated adverse ventricular remodelling post MI via PTEN/AKT signalling [86, 87].

Similarly, IncRNA CAREL was found to be significantly up-regulated in P7 CMs and was linked to reduced CM proliferation. CAREL acts as a competing endogenous ribonucleic acid for miR-296 and de-represses the expression of Trp53inp1 and Itm2a, both inhibitors of CM replication [88]. It is still unclear whether AZIN2sv or CAREL are also expressed by ECs to mediate cardiac regeneration after injury. Interestingly, the foetal LncRNA ECRAR was found to simultaneously promote cardiomyogenesis and angiogenesis post-MI [89]. Even though a paracrine mechanism for increased neovascularization is proposed by the authors, further investigation is required to confirm the therapeutic potential of ECRAR. Recently, the IncRNA GATA6-AS has been reported to silence the expression of GATA6 [90]. GATA6AS is upregulated in ECs during hypoxia inhibiting proliferation of ECs. GATA6 has previously been identified to be involved in CM progenitor cell proliferation [91]. Inhibition of GATA6 through miRNA-10 decreased CM progenitor cell proliferation [91]. The function of GATA6 and its tight regulation through ncRNAs in both ECs and CM progenitor cells indicates the close molecular connection between both cardiac cell types. Identifying key drivers of angiogenesis after $\mathrm{MI}$ can shed light on the way to regenerate the heart. 
Non-coding RNAs in endothelial cell signalling and hypoxia during cardiac regeneration

\section{$\underline{\text { T-UCRS }}$}

During diversion of the human and rodent genomes, 481 regions, ranging from 200 to 779 nts, remained completely conserved. Known as transcribed ultraconserved regions ( $T$ UCRs), these regions are ubiquitously expressed and contain different types of RNA species. T-UCRs are considered essential for gene regulation by acting as antisense transcriptional inhibitors of protein coding and other ncRNA genes in their proximity [3]. Some T-UCRs were described to be dysregulated in disease and, to some extent, have been associated with hypoxia, particularly in cancer, where the tumour microenvironment is typically hypoxic [92]. Moreover, in ischemic stroke, expression of noncoding ultraconserved RNAs seems to be temporally altered in spontaneously hypertensive rats, but the functional significance of these findings is yet to be evaluated [93]. Recently, uc.48+, a IncRNA from a T-UCR, was shown to be upregulated in high-fat diet induced ischemic/reperfused myocardium. Overexpression of uc.48+ enhanced CM apoptosis and myocardial ischemia/reperfusion (MI/R) injury while silencing it had a protective effect. Hence uc.48+ was appointed as a booster of CM apoptosis and MI/R vulnerability to high fat diet [94].

Although the precise functions of T-UCRs are not clear, some of them are known to be modulated by microRNAs in a cross-talk between different classes of ncRNAs [95]. How these new molecular interactions are involved in regulating endothelial and cardiac cell function in pathophysiological conditions remains to be investigated.

\section{Paracrine communication between ECs and CMs}

The intricate network of ECs surrounding CMs in the myocardium allows for molecular interactions to take place during development and disease. The release of ROS during ischaemic injury induces neuregulin-1ßprotein secretion from ECs that promotes CM survival via neuregulin1 $\beta$-erbB4-phosphatidylinositol-3-kinase-Akt pathway [96]. The induction of paracrine neuregulin1 $\beta$-erbB4 signalling between ECs and CMs can also induce $\mathrm{CM}$ proliferation via the activation of the co-receptor ERBB2 [8]. Also, the secretion of PDGF-B and angiopoietin-1 by ECS induces CM protection via the activation of the phosphatidylinositol-3-kinase-Akt pathway. Both factors stimulate EC proliferation and survival in the developing and adult heart [97-99]. Moreover, ECs promote synchronized contraction of CMs and synthesis of the gap junction protein connexin-43 necessary for the electrophysiological coupling of CMs [100].

Targeting the communication between CMs and ECs can be an effective therapeutic tool to prevent $\mathrm{HF}$ and mediate cardiac regeneration. In the last decade, considerable interest has been focused on EVs to explore their potential to promote intercellular crosstalk [101]. EVs are particles that are secreted from cells and contain proteins, mRNAs, and ncRNAs. The content of EVs is released when its membrane comes into contact with the recipient cell, thereby inducing intracellular molecular changes. EVs can be subdivided into categories based on their size and subcellular origin: microvesicles $(0.1-1 \mu \mathrm{m})$, exosomes $(20-100 \mathrm{~nm})$ and apoptotic bodies $(0.5-2 \mu \mathrm{m})$ [101]. All cardiac cells secrete EVs as a mechanism to 
communicate with recipient cells. Exosomal signalling from mesenchymal stem cells (MSCs) during hypoxia promotes EC proliferation and migration [102]. The close anatomical connection between ECs and CMs was a reason to investigate the mechanism of crosstalk in the healthy and diseased heart [103]. Exosomes from CMs were found to modulate the expression of pro-angiogenic genes in ECs. Ischaemic iPSC-derived CMs can produce exosomes that promote EC survival and angiogenesis via RNA-transfer of PIM-1 and VEGF$A$ [104]. HSP70 on the surface of exosomes from cardiac cells was found to induce activation of toll-like receptor- 4 and subsequent ERK1/2 and p38 MAPK signalling increasing CM survival [104].

Depending on the metabolic state of CMs, the miRNA content present in their exosomes is subjected to changes [103]. Consecutively, exosomes from ECs can affect CM contractile function. Exosomal delivery of miR-146a stimulated by an anti-angiogenic 16-kDa Nterminal prolactin fragment has been found to be associated with loss of cardiac function in patients with peripartum cardiomyopathy [105].

A recent study focusing on brain ischemia showed that the miRNA content in exosomes derived from ECs could induce angiogenesis and neurogenesis [106]. CD34 ${ }^{+}$cell-derived exosomes were found to induce angiogenesis and vascular protection through their ncRNA cargo especially miRNA-126 [107-110]. The effect could be enhanced by adding the proangiogenic factor Sonic Hedgehog (Shh) to the exosomes [111]. In the heart, exosomes from healthy CMs were found to contain miRNA-126 that induces proliferation of ECs whereas diabetic CMs contained less miRNA-126 and increased levels of miRNA-320 decreasing angiogenesis [112]. Moreover, ischaemic CM-derived exosomes were found to contain more pro-angiogenic miRNAs than exosomes released by normoxic CMs, suggesting a partial attempt to increase vascularisation in the infarct area [103]. A heterogeneous population of exosomes from CMs could affect the metabolism of recipient cells [113]. Influencing the content of exosomes or stimulating ECs to produce angiogenic, cardioprotective and regenerative factors can provide scope to regenerate the injured myocardium. This highlights the importance of EC-CM communication in multiple cardiovascular diseases and the role of exosomes.

Hence, several pieces of evidence suggest that ncRNAs play an epigenetic role in controlling heart response to hypoxia. Whereas positive regulators are determinant in promoting $\mathrm{CM}$ proliferation and/or neovascularization, negative regulators enhance the deleterious effects of hypoxia. This modulatory role is achieved by regulation of the expression of important hypoxia signalling pathways (e.g. Hif1 $\alpha$ ) and/or secreted molecules (e.g. VEGF). Exosome production and release added further complexity to this regulatory network. Through EVs the effects of hypoxia can be transmitted not only to neighbouring but also to more distant cells, influencing their gene expression. This knowledge was only possible due to technological advances that allowed the study of non-coding elements of the genome. Surely, a better understanding of these mechanisms and how they can be modulated to improve heart response to hypoxia is going to be pivotal for achieving heart regeneration. 


\section{Targeting ECs and extracellular vesicles as a therapeutic tool for heart failure}

Revascularization has been considered as the therapeutic gold standard to treat patients with $\mathrm{MI}$ as endothelial dysfunction is an important aspect towards progression to HF. The growing evidence of EC-CM communication creates a window of opportunity to promote neomyogenesis while targeting ECs. Supplementation with VEGF and angiopoietin-1 was found to concomitantly induce angiogenesis and CM proliferation in a porcine model of $\mathrm{MI}$ [114]. Similarly, injection of human endothelial progenitor cells in a rat model of MI was found to increase neovascularisation and limit infarct size [115].

Furthermore, administration of neuregulin-1 to the injured myocardium, to mimic endothelial cell signalling, has been tested and found beneficial in phase 2 clinical trials [116]. Neuregulin-1 activates endothelial NO synthase in CMs and the serine/threonine kinase Akt [117]. Re-establishing endothelium-CM signalling has also been tested by stimulating the NO-cGMP pathway [118]. NO supplementation was found to simulate the paracrine signalling of ECs and increase $\mathrm{CM}$ integrity and proliferation. Likewise, treatment with serelaxin, the human recombinant form of relaxin- 2 increased NO signalling from ECs [119], also abbreviating endothelial dysfunction after MI. Ultrasound-mediated miRNA126-3p delivery increased EC proliferation and has been since considered of therapeutic interest to treat MI [120]. Inhibition of miRNA-92a expression in a large animal model of ischemia/reperfusion injury induced EC proliferation and improved cardiac function [121]. In addition, modulation of EV content and/or release might be a potential tool to mediate cardiac regeneration. Vesicular communication between cardiac cell types during cardiovascular disease can have detrimental effects on cardiac function. CM-derived TNF $\alpha$ containing EVs induced inflammation and apoptosis of neighbouring CMs [122]. Furthermore, miR-146-loaded vesicles from ECs were found to inhibit angiogenesis of surrounding ECs and inhibit metabolic activity of CMs by targeting Erbb4, Notch1 and Irak1 in peripartum cardiomyopathy [105]. Preventing the paracrine signalling of miR-146 loaded EVs via antimiR-146 ameliorated disease progression. Blocking the delivery of EVs by targeting vesicle release, uptake, or formation has been considered for therapeutic purposes but might have considerable off-target effects (e.g. preventing the secretion of potentially beneficial vesicles) [101]. Specifically blocking the release of miR-21-containing EVs from CFs might be beneficial as these vesicles induce cardiac hypertrophy by downregulating Sorbin and SH3 domain containing 2 (SORBS2) or PDZ and LIM domain 5 (PDLIM5) [123].

Horizontal transfer of ncRNAs via EVs can be modulated to increase the therapeutic potential of ncRNAs and/or of injected cells. In fact, exosomal delivery of miRNA-214 secreted from ECs can induce angiogenesis of neighbouring ECs [124]. Moreover, treatment with vesicles isolated from $\mathrm{CD} 34^{+}$hematopoietic stem cells can mediate $\mathrm{EC}$ proliferation via the transfer of miRNA-126 [107, 108, 110, 125] and vesicles from cardiomyocyte progenitor cells prevented CM apoptosis and promoted EC proliferation [126]. Also, Shh-enriched vesicles were found to effectively induce EC proliferation via the PI3K and ERK-dependent increase of NO [127]. 
Overall these findings indicate that targeting EC signalling via ncRNAs and EVs may serve as a novel therapeutic strategy to treat $\mathrm{MI}$ and HF.

\section{Conclusions}

Ischemic heart disease continues to lead the causes of death despite the large effort of clinicians and academia on reverting this trend [128]. The discovery that the mammalian heart endows an intrinsic, yet transient, regenerative capacity propelled the cardiovascular community to explore the molecular mechanisms governing cardiac regeneration. In this quest, the role of ncRNAs in cardiovascular diseases and cardiac regeneration became evident [5]. Modulating endogenous ncRNAs and cell-to-cell transfer of ncRNAs in a cellspecific manner can serve as a therapeutic tool to induce cardiac regeneration/repair. For a long time, intergenic regions were considered disposable, however their recent discovered value generates new layers of complexity to molecular control of cardiac pathophysiology. Previous studies indicate that neovascularisation is essential for the successful regeneration of the heart. However, hypoxia signalling and hypoxia-activated ncRNAs play an important role in cardiac regeneration. The postnatal metabolic shift towards oxidative phosphorylation, generation of ROS as well as the closure of the ductus arteriosus, resulting in increased blood flow to the left ventricle correlate with the loss of cardiac regenerative capacity $[12,129]$. Nonetheless, hypoxia signalling without subsequent stimulation of angiogenesis does not lead to efficient cardiac regeneration as re-establishment of blood flow to the injured myocardium is required for supporting CMs. In fact, the adult, non-regenerating, mammalian heart is not able to restore the vasculature after MI which worsens myocardial damage and patients with a lower capillary density are more likely to progress to $\mathrm{HF}[130,131]$.

At present, studies on cardiac regeneration either focus on the regeneration of the myocardium or on the regeneration of the cardiac microvasculature. However, the use of single pro-angiogenic agents does not have a substantial effect on cardiac regeneration (reviewed in [2]). The solution might lie in combining pro-angiogenic and neomyogenic factors as well as improving the delivery method (e.g. specific cell targeting via EVs). In fact, multiple lines of evidence suggest that to effectively regenerate the heart, both neovascularisation and CM proliferation need to be promoted in a timely and spatiallydependent manner, as cardiac phenotypes observed in HF are not only attributed to failing CMs but to the whole cellular content.

\section{Future perspectives}

The strong specialization of the adult mammalian heart comes with the cost of low regenerative potential. This low regenerative capacity is on the basis of the heavy socioeconomic burden of cardiovascular diseases. Nevertheless, encouraging reports have found ncRNAs regulating $\mathrm{CM}$ proliferation and neovascularization. The communication between 
ECs and CMs occurs at several levels and these interactions were shown to be central for neonatal cardiac regeneration in mammals. Hence, this connection represents an important opportunity for modulating heart response to injury towards regeneration or improved healing. Future studies must analyse the effect of neovascularisation on CM proliferation and explore the connection between hypoxia, CM proliferation and the timing of the angiogenic response. Moreover, studying the precise changes in the microvasculature and the molecular interaction between the CMs and the ECs during the postnatal period, when the ability to regenerate the heart is lost, can highlight the limiting elements for cardiac regeneration. Knowledge derived from these studies will be of great relevance and will contribute to the development of improved therapeutic approaches.

\section{Acknowledgements}

MMCP was supported by a Netherlands CardioVascular Research Initiative (CVON) grant (REMAIN 2014B027). VSP was supported by a Foundation for Science and Technology of Portugal (FCT) grant (SFRH/BD/111799/2015). PDCM was supported by a Dutch Heart Foundation grant (NHS2015T066) and a Foundation for Science and Technology of Portugal (FCT) grant (PTDC/BIM-MEC/4578/2014).

\section{References}

[1] T.R. Mercer, M.E. Dinger, J.S. Mattick, Long non-coding RNAs: insights into functions, Nature reviews. Genetics 10 (3), 2009, 155-159, https://doi.org/10.1038/nrg2521

[2] R.P. Juni, R.C. Abreu, P.A. da Costa Martins, Regulation of microvascularization in heart failure - an endothelial cell, non-coding RNAs and exosome liaison, Noncoding RNA Res 2 (1), 2017, 45-55, https://doi.org/10.1016/j.ncrna.2017.01.001

[3] G. Bejerano, M. Pheasant, I. Makunin, S. Stephen, W.J. Kent, J.S. Mattick, D. Haussler, Ultraconserved elements in the human genome, Science (New York, N.Y.) 304 (5675), 2004, 1321-1325, https://doi.org/10.1126/science.1098119

[4] N. Papageorgiou, S. Tslamandris, A. Giolis, D. Tousoulis, MicroRNAs in Cardiovascular Disease: Perspectives and Reality, Cardiology in review 24 (3), 2016, 110-118, https://doi.org/10.1097/CRD.0000000000000078

[5] R. Kumarswamy, T. Thum, Non-coding RNAs in cardiac remodeling and heart failure, Circulation research 113 (6), 2013, 676-689, https://doi.org/10.1161/CIRCRESAHA.113.300226

[6] M.Z. Ratajczak, J. Ratajczak, Horizontal transfer of RNA and proteins between cells by extracellular microvesicles: 14 years later, Clinical and translational medicine 5 (1), 2016, 7, https://doi.org/10.1186/s40169016-0087-4

[7] O. Bergmann, R.D. Bhardwaj, S. Bernard, S. Zdunek, F. Barnabe-Heider, S. Walsh, J. Zupicich, K. Alkass, B.A. Buchholz, H. Druid, S. Jovinge, J. Frisen, Evidence for cardiomyocyte renewal in humans, Science (New York, N.Y.) 324 (5923), 2009, 98-102, https://doi.org/10.1126/science.1164680

[8] G. D'Uva, A. Aharonov, M. Lauriola, D. Kain, Y. Yahalom-Ronen, S. Carvalho, K. Weisinger, E. Bassat, D. Rajchman, O. Yifa, M. Lysenko, T. Konfino, J. Hegesh, O. Brenner, M. Neeman, Y. Yarden, J. Leor, R. Sarig, R.P. Harvey, E. Tzahor, ERBB2 triggers mammalian heart regeneration by promoting cardiomyocyte dedifferentiation and proliferation, Nature cell biology 17 (5), 2015, 627-638, https://doi.org/10.1038/ncb3149

[9] E.R. Porrello, A.I. Mahmoud, E. Simpson, J.A. Hill, J.A. Richardson, E.N. Olson, H.A. Sadek, Transient regenerative potential of the neonatal mouse heart, Science (New York, N.Y.) 331 (6020), 2011, 1078-1080, https://doi.org/10.1126/science.1200708

[10] K.D. Poss, L.G. Wilson, M.T. Keating, Heart regeneration in zebrafish, Science (New York, N.Y.) 298 (5601), 2002, 2188-2190, https://doi.org/10.1126/science.1077857 
[11] V. Sampaio-Pinto, S.C. Rodrigues, T.L. Laundos, E.D. Silva, F. Vasques-Novoa, A.C. Silva, R.J. Cerqueira, T.P. Resende, N. Pianca, A. Leite-Moreira, G. D'Uva, S. Thorsteinsdottir, O.P. Pinto-do, D.S. Nascimento, Neonatal Apex Resection Triggers Cardiomyocyte Proliferation, Neovascularization and Functional Recovery Despite Local Fibrosis, Stem cell reports 10 (3), 2018, 860-874, https://doi.org/10.1016/j.stemcr.2018.01.042

[12] B.N. Puente, W. Kimura, S.A. Muralidhar, J. Moon, J.F. Amatruda, K.L. Phelps, D. Grinsfelder, B.A. Rothermel, R. Chen, J.A. Garcia, C.X. Santos, S. Thet, E. Mori, M.T. Kinter, P.M. Rindler, S. Zacchigna, S. Mukherjee, D.J. Chen, A.I. Mahmoud, M. Giacca, P.S. Rabinovitch, A. Aroumougame, A.M. Shah, L.I. Szweda, H.A. Sadek, The oxygen-rich postnatal environment induces cardiomyocyte cell-cycle arrest through DNA damage response, Cell 157 (3), 2014 , 565-579, https://doi.org/10.1016/j.cell.2014.03.032

[13] C. Jopling, E. Sleep, M. Raya, M. Marti, A. Raya, J.C. Izpisua Belmonte, Zebrafish heart regeneration occurs by cardiomyocyte dedifferentiation and proliferation, Nature 464 (7288), 2010, 606-609, https://doi.org/10.1038/nature08899

[14] C. Jopling, G. Sune, A. Faucherre, C. Fabregat, J.C. Izpisua Belmonte, Hypoxia induces myocardial regeneration in zebrafish, Circulation 126 (25), 2012, 3017-3027, https://doi.org/10.1161/CIRCULATIONAHA.112.107888

[15] M.A. Laflamme, C.E. Murry, Heart regeneration, Nature 473 (7347), 2011, 326-335, https://doi.org/10.1038/nature10147

[16] D. Tirziu, F.J. Giordano, M. Simons, Cell communications in the heart, Circulation 122 (9), 2010, 928-937, https://doi.org/10.1161/CIRCULATIONAHA.108.847731

[17] O. Bergmann, S. Zdunek, A. Felker, M. Salehpour, K. Alkass, S. Bernard, S.L. Sjostrom, M. Szewczykowska, T. Jackowska, C. Dos Remedios, T. Malm, M. Andra, R. Jashari, J.R. Nyengaard, G. Possnert, S. Jovinge, H. Druid, J. Frisen, Dynamics of Cell Generation and Turnover in the Human Heart, Cell 161 (7), 2015, 1566-1575, https://doi.org/10.1016/j.cell.2015.05.026

[18] A.R. Pinto, A. Ilinykh, M.J. Ivey, J.T. Kuwabara, M.L. D'Antoni, R. Debuque, A. Chandran, L. Wang, K. Arora, N.A. Rosenthal, M.D. Tallquist, Revisiting Cardiac Cellular Composition, Circulation research 118 (3), 2016, 400-409, https://doi.org/10.1161/CIRCRESAHA.115.307778

[19] S. Doll, M. Dressen, P.E. Geyer, D.N. Itzhak, C. Braun, S.A. Doppler, F. Meier, M.A. Deutsch, H. Lahm, R. Lange, M. Krane, M. Mann, Region and cell-type resolved quantitative proteomic map of the human heart, Nature communications 8 (1), 2017, 1469, https://doi.org/10.1038/s41467-017-01747-2

[20] N. Smart, Prospects for improving neovascularization of the ischemic heart: Lessons from development, Microcirculation (New York, N.Y. : 1994) 24 (1), 2017, https://doi.org/10.1111/micc.12335

[21] Y. Hojo, T. Saito, H. Kondo, Role of apoptosis in left ventricular remodeling after acute myocardial infarction, Journal of cardiology 60 (2), 2012, 91-92, https://doi.org/10.1016/j.jjcc.2012.05.014

[22] P.M. Kang, A. Haunstetter, H. Aoki, A. Usheva, S. Izumo, Morphological and molecular characterization of adult cardiomyocyte apoptosis during hypoxia and reoxygenation, Circulation research 87 (2), 2000, 118-125, https://doi.org/10.1161/01.res.87.2.118

[23] M. Prech, A. Marszalek, J. Schroder, V. Filas, M. Lesiak, M. Jemielity, A. Araszkiewicz, S. Grajek, Apoptosis as a mechanism for the elimination of cardiomyocytes after acute myocardial infarction, The American journal of cardiology 105 (9), 2010, 1240-1245, https://doi.org/10.1016/j.amjcard.2009.12.039

[24] B. Kuhn, F. del Monte, R.J. Hajjar, Y.S. Chang, D. Lebeche, S. Arab, M.T. Keating, Periostin induces proliferation of differentiated cardiomyocytes and promotes cardiac repair, Nature medicine 13 (8), 2007, 962-969, https://doi.org/10.1038/nm1619

[25] W. Kimura, F. Xiao, D.C. Canseco, S. Muralidhar, S. Thet, H.M. Zhang, Y. Abderrahman, R. Chen, J.A. Garcia, J.M. Shelton, J.A. Richardson, A.M. Ashour, A. Asaithamby, H. Liang, C. Xing, Z. Lu, C.C. Zhang, H.A. Sadek, Hypoxia fate mapping identifies cycling cardiomyocytes in the adult heart, Nature 523 (7559), 2015, 226-230, https://doi.org/10.1038/nature14582

[26] C.X. Santos, N. Anilkumar, M. Zhang, A.C. Brewer, A.M. Shah, Redox signaling in cardiac myocytes, Free radical biology \& medicine 50 (7), 2011, 777-793, https://doi.org/10.1016/j.freeradbiomed.2011.01.003

[27] M. Ivan, K. Kondo, H. Yang, W. Kim, J. Valiando, M. Ohh, A. Salic, J.M. Asara, W.S. Lane, W.G. Kaelin, Jr., HIFalpha targeted for VHL-mediated destruction by proline hydroxylation: implications for 02 sensing, Science (New York, N.Y.) 292 (5516), 2001, 464-468, https://doi.org/10.1126/science.1059817

[28] R. Marin-Juez, M. Marass, S. Gauvrit, A. Rossi, S.L. Lai, S.C. Materna, B.L. Black, D.Y. Stainier, Fast revascularization of the injured area is essential to support zebrafish heart regeneration, Proceedings of the National Academy of Sciences of the United States of America 113 (40), 2016, 11237-11242, https://doi.org/10.1073/pnas.1605431113

[29] S. Das, A.B. Goldstone, H. Wang, J. Farry, G. D'Amato, M.J. Paulsen, A. Eskandari, C.E. Hironaka, R. Phansalkar, B. Sharma, S. Rhee, E.A. Shamskhou, D. Agalliu, V. de Jesus Perez, Y.J. Woo, K. Red-Horse, A Unique Collateral Artery Development Program Promotes Neonatal Heart Regeneration, Cell 176 (5), 2019, 1128-1142 e1118, https://doi.org/10.1016/j.cell.2018.12.023 
[30] A. Lepilina, A.N. Coon, K. Kikuchi, J.E. Holdway, R.W. Roberts, C.G. Burns, K.D. Poss, A dynamic epicardial injury response supports progenitor cell activity during zebrafish heart regeneration, Cell 127 (3), 2006, 607-619, https://doi.org/10.1016/j.cell.2006.08.052

[31] J. Fiedler, V. Jazbutyte, B.C. Kirchmaier, S.K. Gupta, J. Lorenzen, D. Hartmann, P. Galuppo, S. Kneitz, J.T. Pena, C. Sohn-Lee, X. Loyer, J. Soutschek, T. Brand, T. Tuschl, J. Heineke, U. Martin, S. Schulte-Merker, G. Ertl, S. Engelhardt, J. Bauersachs, T. Thum, MicroRNA-24 regulates vascularity after myocardial infarction, Circulation 124 (6), 2011, 720-730, https://doi.org/10.1161/CIRCULATIONAHA.111.039008

[32] J.W. Kornfeld, J.C. Bruning, Regulation of metabolism by long, non-coding RNAs, Frontiers in genetics 5 2014, 57, https://doi.org/10.3389/fgene.2014.00057

[33] S. Rane, M. He, D. Sayed, H. Vashistha, A. Malhotra, J. Sadoshima, D.E. Vatner, S.F. Vatner, M. Abdellatif, Downregulation of miR-199a derepresses hypoxia-inducible factor-1alpha and Sirtuin 1 and recapitulates hypoxia preconditioning in cardiac myocytes, Circulation research 104 (7), 2009, 879-886, https://doi.org/10.1161/CIRCRESAHA.108.193102

[34] J. Fiedler, K. Breckwoldt, C.W. Remmele, D. Hartmann, M. Dittrich, A. Pfanne, A. Just, K. Xiao, M. Kunz, T. Muller, A. Hansen, R. Geffers, T. Dandekar, T. Eschenhagen, T. Thum, Development of Long Noncoding RNA-Based Strategies to Modulate Tissue Vascularization, J Am Coll Cardiol 66 (18), 2015, 2005-2015, https://doi.org/10.1016/j.jacc.2015.07.081

[35] A. Eulalio, M. Mano, M. Dal Ferro, L. Zentilin, G. Sinagra, S. Zacchigna, M. Giacca, Functional screening identifies miRNAs inducing cardiac regeneration, Nature 492 (7429), 2012, 376-381, https://doi.org/10.1038/nature11739

[36] J.F. Chen, E.P. Murchison, R. Tang, T.E. Callis, M. Tatsuguchi, Z. Deng, M. Rojas, S.M. Hammond, M.D. Schneider, C.H. Selzman, G. Meissner, C. Patterson, G.J. Hannon, D.Z. Wang, Targeted deletion of Dicer in the heart leads to dilated cardiomyopathy and heart failure, Proceedings of the National Academy of Sciences of the United States of America 105 (6), 2008, 2111-2116, https://doi.org/10.1073/pnas.0710228105

[37] P.K. Rao, Y. Toyama, H.R. Chiang, S. Gupta, M. Bauer, R. Medvid, F. Reinhardt, R. Liao, M. Krieger, R. Jaenisch, H.F. Lodish, R. Blelloch, Loss of cardiac microRNA-mediated regulation leads to dilated cardiomyopathy and heart failure, Circulation research 105 (6), 2009, 585-594, https://doi.org/10.1161/CIRCRESAHA.109.200451

[38] Y. Suarez, C. Fernandez-Hernando, J.S. Pober, W.C. Sessa, Dicer dependent microRNAs regulate gene expression and functions in human endothelial cells, Circulation research 100 (8), 2007, 1164-1173, https://doi.org/10.1161/01.RES.0000265065.26744.17

[39] W.J. Yang, D.D. Yang, S. Na, G.E. Sandusky, Q. Zhang, G. Zhao, Dicer is required for embryonic angiogenesis during mouse development, The Journal of biological chemistry 280 (10), 2005, 9330-9335, https://doi.org/10.1074/jbc.M413394200

[40] H.E. Azzouzi, S. Leptidis, P.A. Doevendans, L.J. De Windt, HypoxamiRs: regulators of cardiac hypoxia and energy metabolism, Trends in endocrinology and metabolism: TEM 26 (9), 2015, 502-508, https://doi.org/10.1016/j.tem.2015.06.008

[41] Z. Hua, Q. Lv, W. Ye, C.K. Wong, G. Cai, D. Gu, Y. Ji, C. Zhao, J. Wang, B.B. Yang, Y. Zhang, MiRNA-directed regulation of VEGF and other angiogenic factors under hypoxia, PloS one 1 2006, e116, https://doi.org/10.1371/journal.pone.0000116

[42] H.T. Deng, H.L. Liu, B.B. Zhai, K. Zhang, G.C. Xu, X.M. Peng, Q.Z. Zhang, L.Y. Li, Vascular endothelial growth factor suppresses TNFSF15 production in endothelial cells by stimulating miR-31 and miR-20a expression via activation of Akt and Erk signals, FEBS open bio 7 (1), 2017, 108-117, https://doi.org/10.1002/2211-5463.12171

[43] M. Arif, R. Pandey, P. Alam, S. Jiang, S. Sadayappan, A. Paul, R.P.H. Ahmed, MicroRNA-210-mediated proliferation, survival, and angiogenesis promote cardiac repair post myocardial infarction in rodents, Journal of molecular medicine (Berlin, Germany) 95 (12), 2017, 1369-1385, https://doi.org/10.1007/s00109-017-1591-8

[44] S. Greco, C. Gaetano, F. Martelli, HypoxamiR regulation and function in ischemic cardiovascular diseases, Antioxidants \& redox signaling 21 (8), 2014, 1202-1219, https://doi.org/10.1089/ars.2013.5403

[45] G. Zaccagnini, B. Maimone, V. Di Stefano, P. Fasanaro, S. Greco, A. Perfetti, M.C. Capogrossi, C. Gaetano, F. Martelli, Hypoxia-induced miR-210 modulates tissue response to acute peripheral ischemia, Antioxidants \& redox signaling 21 (8), 2014, 1177-1188, https://doi.org/10.1089/ars.2013.5206

[46] G. Ghosh, I.V. Subramanian, N. Adhikari, X. Zhang, H.P. Joshi, D. Basi, Y.S. Chandrashekhar, J.L. Hall, S. Roy, Y. Zeng, S. Ramakrishnan, Hypoxia-induced microRNA-424 expression in human endothelial cells regulates HIF-alpha isoforms and promotes angiogenesis, The Journal of clinical investigation 120 (11), 2010, 4141-4154, https://doi.org/10.1172/JCl42980

[47] S. Hu, M. Huang, Z. Li, F. Jia, Z. Ghosh, M.A. Lijkwan, P. Fasanaro, N. Sun, X. Wang, F. Martelli, R.C. Robbins, J.C. Wu, MicroRNA-210 as a novel therapy for treatment of ischemic heart disease, Circulation 122 (11 Suppl), 2010, S124-131, https://doi.org/10.1161/CIRCULATIONAHA.109.928424 
[48] Y. Wang, X. Pan, Y. Fan, X. Hu, X. Liu, M. Xiang, J. Wang, Dysregulated expression of microRNAs and mRNAs in myocardial infarction, American journal of translational research 7 (11), 2015, 2291-2304,

[49] H. Nishi, K. Ono, Y. Iwanaga, T. Horie, K. Nagao, G. Takemura, M. Kinoshita, Y. Kuwabara, R.T. Mori, K. Hasegawa, T. Kita, T. Kimura, MicroRNA-15b modulates cellular ATP levels and degenerates mitochondria via Arl2 in neonatal rat cardiac myocytes, The Journal of biological chemistry 285 (7), 2010, 4920-4930, https://doi.org/10.1074/jbc.M109.082610

[50] E.M. Small, R.J. Frost, E.N. Olson, MicroRNAs add a new dimension to cardiovascular disease, Circulation 121 (8), 2010, 1022-1032, https://doi.org/10.1161/CIRCULATIONAHA.109.889048

[51] A. Chamorro-Jorganes, E. Araldi, L.O. Penalva, D. Sandhu, C. Fernandez-Hernando, Y. Suarez, MicroRNA-16 and microRNA-424 regulate cell-autonomous angiogenic functions in endothelial cells via targeting vascular endothelial growth factor receptor-2 and fibroblast growth factor receptor-1, Arteriosclerosis, thrombosis, and vascular biology 31 (11), 2011, 2595-2606, https://doi.org/10.1161/ATVBAHA.111.236521

[52] E.R. Porrello, A.I. Mahmoud, E. Simpson, B.A. Johnson, D. Grinsfelder, D. Canseco, P.P. Mammen, B.A. Rothermel, E.N. Olson, H.A. Sadek, Regulation of neonatal and adult mammalian heart regeneration by the miR15 family, Proceedings of the National Academy of Sciences of the United States of America 110 (1), 2013, 187192, https://doi.org/10.1073/pnas.1208863110

[53] T.G. Hullinger, R.L. Montgomery, A.G. Seto, B.A. Dickinson, H.M. Semus, J.M. Lynch, C.M. Dalby, K. Robinson, C. Stack, P.A. Latimer, J.M. Hare, E.N. Olson, E. van Rooij, Inhibition of miR-15 protects against cardiac ischemic injury, Circulation research 110 (1), 2012, 71-81, https://doi.org/10.1161/CIRCRESAHA.111.244442

[54] G.H. Dai, P.Z. Ma, X.B. Song, N. Liu, T. Zhang, B. Wu, MicroRNA-223-3p inhibits the angiogenesis of ischemic cardiac microvascular endothelial cells via affecting RPS6KB1/hif-1a signal pathway, PloS one 9 (10), 2014, e108468, https://doi.org/10.1371/journal.pone.0108468

[55] B. Icli, A.K. Wara, J. Moslehi, X. Sun, E. Plovie, M. Cahill, J.F. Marchini, A. Schissler, R.F. Padera, J. Shi, H.W. Cheng, S. Raghuram, Z. Arany, R. Liao, K. Croce, C. MacRae, M.W. Feinberg, MicroRNA-26a regulates pathological and physiological angiogenesis by targeting BMP/SMAD1 signaling, Circulation research 113 (11), 2013, 1231-1241, https://doi.org/10.1161/CIRCRESAHA.113.301780

[56] M. Meloni, M. Marchetti, K. Garner, B. Littlejohns, G. Sala-Newby, N. Xenophontos, I. Floris, M.S. Suleiman, P. Madeddu, A. Caporali, C. Emanueli, Local inhibition of microRNA-24 improves reparative angiogenesis and left ventricle remodeling and function in mice with myocardial infarction, Molecular therapy : the journal of the American Society of Gene Therapy 21 (7), 2013, 1390-1402, https://doi.org/10.1038/mt.2013.89

[57] A. Magenta, C. Cencioni, P. Fasanaro, G. Zaccagnini, S. Greco, G. Sarra-Ferraris, A. Antonini, F. Martelli, M.C. Capogrossi, miR-200c is upregulated by oxidative stress and induces endothelial cell apoptosis and senescence via ZEB1 inhibition, Cell Death Differ 18 (10), 2011, 1628-1639, https://doi.org/10.1038/cdd.2011.42

[58] N. Papageorgiou, E. Zacharia, D. Tousoulis, Association between microRNAs and coronary collateral circulation: is there a new role for the small non-coding RNAs?, Annals of translational medicine 4 (11), 2016, 223, https://doi.org/10.21037/atm.2016.05.51

[59] K. Chen, W. Fan, X. Wang, X. Ke, G. Wu, C. Hu, MicroRNA-101 mediates the suppressive effect of laminar shear stress on mTOR expression in vascular endothelial cells, Biochemical and biophysical research communications 427 (1), 2012, 138-142, https://doi.org/10.1016/j.bbrc.2012.09.026

[60] S. Grundmann, F.P. Hans, S. Kinniry, J. Heinke, T. Helbing, F. Bluhm, J.P. Sluijter, I. Hoefer, G. Pasterkamp, C. Bode, M. Moser, MicroRNA-100 regulates neovascularization by suppression of mammalian target of rapamycin in endothelial and vascular smooth muscle cells, Circulation 123 (9), 2011, 999-1009, https://doi.org/10.1161/CIRCULATIONAHA.110.000323

[61] J.H. Kim, K.S. Lee, D.K. Lee, J. Kim, S.N. Kwak, K.S. Ha, J. Choe, M.H. Won, B.R. Cho, D. Jeoung, H. Lee, Y.G. Kwon, Y.M. Kim, Hypoxia-responsive microRNA-101 promotes angiogenesis via heme oxygenase-1/vascular endothelial growth factor axis by targeting cullin 3, Antioxidants \& redox signaling 21 (18), 2014, 2469-2482, https://doi.org/10.1089/ars.2014.5856

[62] S. Wang, A.B. Aurora, B.A. Johnson, X. Qi, J. McAnally, J.A. Hill, J.A. Richardson, R. Bassel-Duby, E.N. Olson, The endothelial-specific microRNA miR-126 governs vascular integrity and angiogenesis, Developmental cell 15 (2), 2008, 261-271, https://doi.org/10.1016/j.devcel.2008.07.002

[63] J.E. Fish, M.M. Santoro, S.U. Morton, S. Yu, R.F. Yeh, J.D. Wythe, K.N. Ivey, B.G. Bruneau, D.Y. Stainier, D. Srivastava, miR-126 regulates angiogenic signaling and vascular integrity, Developmental cell 15 (2), 2008, 272284, https://doi.org/10.1016/j.devcel.2008.07.008

[64] J. Semo, R. Sharir, A. Afek, C. Avivi, I. Barshack, S. Maysel-Auslender, Y. Krelin, D. Kain, M. Entin-Meer, G. Keren, J. George, The 106b approximately 25 microRNA cluster is essential for neovascularization after hindlimb ischaemia in mice, European heart journal 35 (45), 2014, 3212-3223, https://doi.org/10.1093/eurheartj/eht041 [65] A. Haghikia, E. Missol-Kolka, D. Tsikas, L. Venturini, S. Brundiers, M. Castoldi, M.U. Muckenthaler, M. Eder, B. Stapel, T. Thum, A. Haghikia, E. Petrasch-Parwez, H. Drexler, D. Hilfiker-Kleiner, M. Scherr, Signal transducer and 
activator of transcription 3-mediated regulation of miR-199a-5p links cardiomyocyte and endothelial cell function in the heart: a key role for ubiquitin-conjugating enzymes, European heart journal 32 (10), 2011, 1287-1297, https://doi.org/10.1093/eurheartj/ehq369

[66] L. Ottaviani, R.P. Juni, J. Halkein, H. el Azzouzi, S. Olieslagers, L.J. de Windt, P.A. da Costa Martins, Cardiomyocyte-derived exosomes mediate pathological cardiac microvascular remodeling, Journal of molecular and cellular cardiology 120 2018, 45, https://doi.org/10.1016/j.yjmcc.2018.05.134

[67] X. Wang, W. Huang, G. Liu, W. Cai, R.W. Millard, Y. Wang, J. Chang, T. Peng, G.C. Fan, Cardiomyocytes mediate anti-angiogenesis in type 2 diabetic rats through the exosomal transfer of miR-320 into endothelial cells, Journal of molecular and cellular cardiology 74 2014, 139-150, https://doi.org/10.1016/j.yjmcc.2014.05.001

[68] D. Sabour, R.S.R. Machado, J.P. Pinto, S. Rohani, R.G.A. Sahito, J. Hescheler, M.E. Futschik, A. Sachinidis, Parallel Genome-wide Profiling of Coding and Non-coding RNAs to Identify Novel Regulatory Elements in Embryonic and Maturated Heart, Molecular therapy. Nucleic acids 12 2018, 158-173, https://doi.org/10.1016/j.omtn.2018.04.018

[69] S. Yang, T. Fan, Q. Hu, W. Xu, J. Yang, C. Xu, B. Zhang, J. Chen, H. Jiang, Downregulation of microRNA-17-5p improves cardiac function after myocardial infarction via attenuation of apoptosis in endothelial cells, Molecular genetics and genomics : MGG 293 (4), 2018, 883-894, https://doi.org/10.1007/s00438-018-1426-5

[70] M. Adamowicz, C.C. Morgan, B.J. Haubner, M. Noseda, M.J. Collins, M.A. Paiva, P.K. Srivastava, P. Gellert, B. Razzaghi, P. O'Gara, P. Raina, L. Game, L. Bottolo, M.D. Schneider, S.E. Harding, J. Penninger, T.J. Aitman, Functionally Conserved Noncoding Regulators of Cardiomyocyte Proliferation and Regeneration in Mouse and Human, Circulation-Genomic and Precision Medicine 11 (2), 2018, e001805, https://doi.org/ARTN e001805

[71] J.C. Scheuermann, L.A. Boyer, Getting to the heart of the matter: long non-coding RNAs in cardiac development and disease, The EMBO journal 32 (13), 2013, 1805-1816, https://doi.org/10.1038/emboj.2013.134

[72] M.D. Ballantyne, R.A. McDonald, A.H. Baker, IncRNA/MicroRNA interactions in the vasculature, Clinical pharmacology and therapeutics 99 (5), 2016, 494-501, https://doi.org/10.1002/cpt.355

[73] M.S. Ebert, P.A. Sharp, Emerging roles for natural microRNA sponges, Current biology : CB 20 (19), 2010, R858861, https://doi.org/10.1016/j.cub.2010.08.052

[74] K. Wang, B. Long, L.Y. Zhou, F. Liu, Q.Y. Zhou, C.Y. Liu, Y.Y. Fan, P.F. Li, CARL IncRNA inhibits anoxia-induced mitochondrial fission and apoptosis in cardiomyocytes by impairing miR-539-dependent PHB2 downregulation, Nature communications 5 2014, 3596, https://doi.org/10.1038/ncomms4596

[75] M.S. Leisegang, C. Fork, I. Josipovic, F.M. Richter, J. Preussner, J. Hu, M.J. Miller, J. Epah, P. Hofmann, S. Gunther, F. Moll, C. Valasarajan, J. Heidler, Y. Ponomareva, T.M. Freiman, L. Maegdefessel, K.H. Plate, M. Mittelbronn, S. Uchida, C. Kunne, K. Stellos, R.T. Schermuly, N. Weissmann, K. Devraj, I. Wittig, R.A. Boon, S. Dimmeler, S.S. Pullamsetti, M. Looso, F.J. Miller, Jr., R.P. Brandes, Long Noncoding RNA MANTIS Facilitates $\begin{array}{llllll}\text { Endothelial Angiogenic } \quad \text { Function, } & \text { Circulation } & \text { (1), 2017, }\end{array}$ https://doi.org/10.1161/CIRCULATIONAHA.116.026991

[76] I. Josipovic, C. Fork, J. Preussner, K.K. Prior, D. Iloska, A.E. Vasconez, S. Labocha, C. Angioni, D. Thomas, N. Ferreiros, M. Looso, S.S. Pullamsetti, G. Geisslinger, D. Steinhilber, R.P. Brandes, M.S. Leisegang, PAFAH1B1 and the IncRNA NONHSAT073641 maintain an angiogenic phenotype in human endothelial cells, Acta physiologica (Oxford, England) 218 (1), 2016, 13-27, https://doi.org/10.1111/apha.12700

[77] N. Ishii, K. Ozaki, H. Sato, H. Mizuno, S. Saito, A. Takahashi, Y. Miyamoto, S. Ikegawa, N. Kamatani, M. Hori, S. Saito, Y. Nakamura, T. Tanaka, Identification of a novel non-coding RNA, MIAT, that confers risk of myocardial infarction, Journal of human genetics 51 (12), 2006, 1087-1099, https://doi.org/10.1007/s10038-006-0070-9

[78] B. Yan, J. Yao, J.Y. Liu, X.M. Li, X.Q. Wang, Y.J. Li, Z.F. Tao, Y.C. Song, Q. Chen, Q. Jiang, IncRNA-MIAT regulates microvascular dysfunction by functioning as a competing endogenous RNA, Circulation research 116 (7), 2015, 1143-1156, https://doi.org/10.1161/CIRCRESAHA.116.305510

[79] L. Kurian, A. Aguirre, I. Sancho-Martinez, C. Benner, T. Hishida, T.B. Nguyen, P. Reddy, E. Nivet, M.N. Krause, D.A. Nelles, C.R. Esteban, J.M. Campistol, G.W. Yeo, J.C.I. Belmonte, Identification of novel long noncoding RNAs underlying vertebrate cardiovascular development, Circulation 131 (14), 2015, 1278-1290, https://doi.org/10.1161/CIRCULATIONAHA.114.013303

[80] J. Hou, C. Zhou, H. Long, S. Zheng, T. Guo, Q. Wu, H. Wu, T. Zhong, T. Wang, Long noncoding RNAs: Novel molecules in cardiovascular biology, disease and regeneration, Experimental and molecular pathology 100 (3), 2016, 493-501, https://doi.org/10.1016/j.yexmp.2016.05.006

[81] K.M. Michalik, X. You, Y. Manavski, A. Doddaballapur, M. Zornig, T. Braun, D. John, Y. Ponomareva, W. Chen, S. Uchida, R.A. Boon, S. Dimmeler, Long noncoding RNA MALAT1 regulates endothelial cell function and vessel growth, Circulation research 114 (9), 2014, 1389-1397, https://doi.org/10.1161/CIRCRESAHA.114.303265

[82] H.S.J. Man, A.N. Sukumar, G.C. Lam, P.J. Turgeon, M.S. Yan, K.H. Ku, M.K. Dubinsky, J.J.D. Ho, J.J. Wang, S. Das, N. Mitchell, P. Oettgen, M.V. Sefton, P.A. Marsden, Angiogenic patterning by STEEL, an endothelial-enriched long 
noncoding RNA, Proceedings of the National Academy of Sciences of the United States of America 115 (10), 2018, 2401-2406, https://doi.org/10.1073/pnas.1715182115

[83] Y. Miao, N.E. Ajami, T.S. Huang, F.M. Lin, C.H. Lou, Y.T. Wang, S. Li, J. Kang, H. Munkacsi, M.R. Maurya, S. Gupta, S. Chien, S. Subramaniam, Z. Chen, Enhancer-associated Iong non-coding RNA LEENE regulates endothelial nitric oxide synthase and endothelial function, Nature communications 9 (1), 2018, 292, https://doi.org/10.1038/s41467-017-02113-y

[84] B. Liao, R. Chen, F. Lin, A. Mai, J. Chen, H. Li, Z. Xu, S. Dong, Long noncoding RNA HOTTIP promotes endothelial cell proliferation and migration via activation of the $\mathrm{Wnt} /$ beta-catenin pathway, Journal of cellular biochemistry 119 (3), 2018, 2797-2805, https://doi.org/10.1002/jcb.26448

[85] R. Kumarswamy, C. Bauters, I. Volkmann, F. Maury, J. Fetisch, A. Holzmann, G. Lemesle, P. de Groote, F. Pinet, T. Thum, Circulating long noncoding RNA, LIPCAR, predicts survival in patients with heart failure, Circulation research 114 (10), 2014, 1569-1575, https://doi.org/10.1161/CIRCRESAHA.114.303915

[86] Z. Wu, S. Zhao, C. Li, C. Liu, LncRNA TUG1 serves an important role in hypoxia-induced myocardial cell injury by regulating the miR1455pBinp3 axis, Molecular medicine reports 17 (2), 2018, 2422-2430, https://doi.org/10.3892/mmr.2017.8116

[87] X. Li, X. He, H. Wang, M. Li, S. Huang, G. Chen, Y. Jing, S. Wang, Y. Chen, W. Liao, Y. Liao, J. Bin, Loss of AZIN2 splice variant facilitates endogenous cardiac regeneration, Cardiovascular research 114 (12), 2018, 1642-1655, https://doi.org/10.1093/cvr/cvy075

[88] B. Cai, W. Ma, F. Ding, L. Zhang, Q. Huang, X. Wang, B. Hua, J. Xu, J. Li, C. Bi, S. Guo, F. Yang, Z. Han, Y. Li, G. Yan, Y. Yu, Z. Bao, M. Yu, F. Li, Y. Tian, Z. Pan, B. Yang, The Long Noncoding RNA CAREL Controls Cardiac Regeneration, J Am Coll Cardiol 72 (5), 2018, 534-550, https://doi.org/10.1016/j.jacc.2018.04.085

[89] Y. Chen, X. Li, B. Li, H. Wang, M. Li, S. Huang, Y. Sun, G. Chen, X. Si, C. Huang, W. Liao, Y. Liao, J. Bin, Long Noncoding RNA ECRAR Triggers Post-natal Myocardial Regeneration by Activating ERK1/2 Signaling, Molecular therapy : the journal of the American Society of Gene Therapy 27 (1), 2019, 29-45, https://doi.org/10.1016/j.ymthe.2018.10.021

[90] P. Neumann, N. Jae, A. Knau, S.F. Glaser, Y. Fouani, O. Rossbach, M. Kruger, D. John, A. Bindereif, P. Grote, R.A. Boon, S. Dimmeler, The IncRNA GATA6-AS epigenetically regulates endothelial gene expression via interaction with LOXL2, Nature communications 9 (1), 2018, 237, https://doi.org/10.1038/s41467-017-02431-1

[91] D. Liang, L. Zhen, T. Yuan, J. Huang, F. Deng, Wuyahan, H. Zhang, L. Pan, Y. Liu, E. The, Z. Yu, W. Zhu, Y. Zhang, L. Li, L. Peng, J. Li, Y.H. Chen, miR-10a regulates proliferation of human cardiomyocyte progenitor cells by targeting GATA6, PloS one 9 (7), 2014, e103097, https://doi.org/10.1371/journal.pone.0103097

[92] J. Ferdin, N. Nishida, X. Wu, M.S. Nicoloso, M.Y. Shah, C. Devlin, H. Ling, M. Shimizu, K. Kumar, M.A. Cortez, M. Ferracin, Y. Bi, D. Yang, B. Czerniak, W. Zhang, T.D. Schmittgen, M.P. Voorhoeve, M.J. Reginato, M. Negrini, R.V. Davuluri, T. Kunej, M. Ivan, G.A. Calin, HINCUTs in cancer: hypoxia-induced noncoding ultraconserved transcripts, Cell Death Differ 20 (12), 2013, 1675-1687, https://doi.org/10.1038/cdd.2013.119

[93] S.L. Mehta, R. Vemuganti, Ischemic Stroke Alters the Expression of the Transcribed Ultraconserved Regions of the Genome in Rat Brain, Stroke 49 (4), 2018, 1024-1028, https://doi.org/10.1161/STROKEAHA.118.020663

[94] L. Ding, C. Gong, J. Zhao, X. Liu, T. Li, S. Rao, S. Wang, Y. Liu, S. Peng, W. Xiao, C. Xiong, R. Wang, S. Liang, H. $\mathrm{Xu}$, Noncoding transcribed ultraconserved region (T-UCR) UC.48+ is a novel regulator of high-fat diet induced myocardial ischemia/reperfusion injury, Journal of cellular physiology 234 (6), 2019, 9849-9861, https://doi.org/10.1002/jcp.27674

[95] S. Terreri, M. Durso, V. Colonna, A. Romanelli, D. Terracciano, M. Ferro, S. Perdona, L. Castaldo, F. Febbraio, F. de Nigris, A. Cimmino, New Cross-Talk Layer between Ultraconserved Non-Coding RNAs, MicroRNAs and Polycomb Protein YY1 in Bladder Cancer, Genes 7 (12), 2016, https://doi.org/10.3390/genes7120127

[96] Y. Kuramochi, G.M. Cote, X. Guo, N.K. Lebrasseur, L. Cui, R. Liao, D.B. Sawyer, Cardiac endothelial cells regulate reactive oxygen species-induced cardiomyocyte apoptosis through neuregulin-1beta/erbB4 signaling, The Journal of biological chemistry 279 (49), 2004, 51141-51147, https://doi.org/10.1074/jbc.M408662200

[97] S.M. Dallabrida, N. Ismail, J.R. Oberle, B.E. Himes, M.A. Rupnick, Angiopoietin-1 promotes cardiac and skeletal myocyte survival through integrins, Circulation research 96 (4), 2005, e8-24, https://doi.org/10.1161/01.RES.0000158285.57191.60

[98] J.M. Edelberg, W.C. Aird, W. Wu, H. Rayburn, W.S. Mamuya, M. Mercola, R.D. Rosenberg, PDGF mediates cardiac microvascular communication, The Journal of clinical investigation 102 (4), 1998, 837-843, https://doi.org/10.1172/JCl3058

[99] J.M. Edelberg, S.H. Lee, M. Kaur, L. Tang, N.M. Feirt, S. McCabe, O. Bramwell, S.C. Wong, M.K. Hong, Plateletderived growth factor- $A B$ limits the extent of myocardial infarction in a rat model: feasibility of restoring impaired angiogenic capacity in the aging heart, Circulation 105 (5), 2002, 608-613, https://doi.org/10.1161/hc0502.103672 
[100] D.A. Narmoneva, R. Vukmirovic, M.E. Davis, R.D. Kamm, R.T. Lee, Endothelial cells promote cardiac myocyte survival and spatial reorganization: implications for cardiac regeneration, Circulation 110 (8), 2004, 962-968, https://doi.org/10.1161/01.CIR.0000140667.37070.07

[101] E.L.A. S, I. Mager, X.O. Breakefield, M.J. Wood, Extracellular vesicles: biology and emerging therapeutic opportunities, Nature reviews. Drug discovery 12 (5), 2013, 347-357, https://doi.org/10.1038/nrd3978

[102] C. Salomon, J. Ryan, L. Sobrevia, M. Kobayashi, K. Ashman, M. Mitchell, G.E. Rice, Exosomal signaling during hypoxia mediates microvascular endothelial cell migration and vasculogenesis, Plos one 8 (7), 2013, e68451, https://doi.org/10.1371/journal.pone.0068451

[103] N.A. Garcia, I. Ontoria-Oviedo, H. Gonzalez-King, A. Diez-Juan, P. Sepulveda, Glucose Starvation in Cardiomyocytes Enhances Exosome Secretion and Promotes Angiogenesis in Endothelial Cells, PloS one 10 (9), 2015, e0138849, https://doi.org/10.1371/journal.pone.0138849

[104] S. Malepu, H. Renikunta, N. Kraenkel, A. Kratzer, H. Giral, G. Hasenfuss, H. Stachelscheid, K. StreckfussBoemeke, U. Landmesser, P. Jakob, Ischemia-driven exosome release of human iPSC-CM-derived cardiomyocytes increase viability of endothelial cells via pro-survival factors, European heart journal 38 (suppl_1), 2017, https://doi.org/10.1093/eurheartj/ehx493.5918

[105] J. Halkein, S.P. Tabruyn, M. Ricke-Hoch, A. Haghikia, N.Q. Nguyen, M. Scherr, K. Castermans, L. Malvaux, V. Lambert, M. Thiry, K. Sliwa, A. Noel, J.A. Martial, D. Hilfiker-Kleiner, I. Struman, MicroRNA-146a is a therapeutic target and biomarker for peripartum cardiomyopathy, The Journal of clinical investigation 123 (5), 2013, 21432154, https://doi.org/10.1172/JCl64365

[106] X. Liu, W. Pan, C. Li, R. Zhang, B. Fan, X. Wang, X. Zhang, J.N. Hu, M. Chopp, Z.G. Zhang, Abstract 177: Exosomal Micrornas From Ischemic Cerebral Endothelial Cells and Neural Stem Cells Regulate Coupling of Neurogenesis and Angiogenesis, Stroke 49 (Suppl_1), 2018, A177-A177, https://doi.org/10.1161/str.49.suppl_1.177

[107] L. Chen, J. Wang, B. Wang, J. Yang, Z. Gong, X. Zhao, C. Zhang, K. Du, MiR-126 inhibits vascular endothelial cell apoptosis through targeting PI3K/Akt signaling, Annals of hematology 95 (3), 2016, 365-374, https://doi.org/10.1007/s00277-015-2567-9

[108] P. Mathiyalagan, Y. Liang, D. Kim, S. Misener, T. Thorne, C.E. Kamide, E. Klyachko, D.W. Losordo, R.J. Hajjar, S. Sahoo, Angiogenic Mechanisms of Human CD34(+) Stem Cell Exosomes in the Repair of Ischemic Hindlimb, Circulation research 120 (9), 2017, 1466-1476, https://doi.org/10.1161/CIRCRESAHA.116.310557

[109] S. Sahoo, E. Klychko, T. Thorne, S. Misener, K.M. Schultz, M. Millay, A. Ito, T. Liu, C. Kamide, H. Agrawal, H. Perlman, G. Qin, R. Kishore, D.W. Losordo, Exosomes from human CD34(+) stem cells mediate their proangiogenic paracrine activity, Circulation research 109 (7), 2011, 724-728, https://doi.org/10.1161/CIRCRESAHA.111.253286 [110] A. Zernecke, K. Bidzhekov, H. Noels, E. Shagdarsuren, L. Gan, B. Denecke, M. Hristov, T. Koppel, M.N. Jahantigh, E. Lutgens, S. Wang, E.N. Olson, A. Schober, C. Weber, Delivery of microRNA-126 by apoptotic bodies induces CXCL12-dependent vascular protection, Science signaling 2 (100), 2009, ra81, https://doi.org/10.1126/scisignal.2000610

[111] A.R. Mackie, E. Klyachko, T. Thorne, K.M. Schultz, M. Millay, A. Ito, C.E. Kamide, T. Liu, R. Gupta, S. Sahoo, S. Misener, R. Kishore, D.W. Losordo, Sonic hedgehog-modified human CD34+ cells preserve cardiac function after acute myocardial infarction, Circulation research 111 (3), 2012, 312-321, https://doi.org/10.1161/CIRCRESAHA.112.266015

[112] E.S.B. Salem, G.C. Fan, Pathological Effects of Exosomes in Mediating Diabetic Cardiomyopathy, Advances in experimental medicine and biology 998 2017, 113-138, https://doi.org/10.1007/978-981-10-4397-0_8

[113] A. Waldenstrom, N. Genneback, U. Hellman, G. Ronquist, Cardiomyocyte microvesicles contain DNA/RNA and convey biological messages to target cells, PloS one 7 (4), 2012, e34653, https://doi.org/10.1371/journal.pone.0034653

[114] Z. Tao, B. Chen, X. Tan, Y. Zhao, L. Wang, T. Zhu, K. Cao, Z. Yang, Y.W. Kan, H. Su, Coexpression of VEGF and angiopoietin-1 promotes angiogenesis and cardiomyocyte proliferation reduces apoptosis in porcine myocardial infarction (MI) heart, Proceedings of the National Academy of Sciences of the United States of America 108 (5), 2011, 2064-2069, https://doi.org/10.1073/pnas.1018925108

[115] A. Kawamoto, H.C. Gwon, H. Iwaguro, J.I. Yamaguchi, S. Uchida, H. Masuda, M. Silver, H. Ma, M. Kearney, J.M. Isner, T. Asahara, Therapeutic potential of ex vivo expanded endothelial progenitor cells for myocardial ischemia, Circulation 103 (5), 2001, 634-637, https://doi.org/10.1161/01.cir.103.5.634

[116] R. Gao, J. Zhang, L. Cheng, X. Wu, W. Dong, X. Yang, T. Li, X. Liu, Y. Xu, X. Li, M. Zhou, A Phase II, randomized, double-blind, multicenter, based on standard therapy, placebo-controlled study of the efficacy and safety of recombinant human neuregulin-1 in patients with chronic heart failure, J Am Coll Cardiol 55 (18), 2010, 1907-1914, https://doi.org/10.1016/j.jacc.2009.12.044 
[117] K. Lemmens, P. Fransen, S.U. Sys, D.L. Brutsaert, G.W. De Keulenaer, Neuregulin-1 induces a negative inotropic effect in cardiac muscle: role of nitric oxide synthase, Circulation 109 (3), 2004, 324-326, https://doi.org/10.1161/01.CIR.0000114521.88547.5E

[118] E. Lepic, D. Burger, X. Lu, W. Song, Q. Feng, Lack of endothelial nitric oxide synthase decreases cardiomyocyte proliferation and delays cardiac maturation, American journal of physiology. Cell physiology 291 (6), 2006, C12401246, https://doi.org/10.1152/ajpcell.00092.2006

[119] T. Dschietzig, A. Brecht, C. Bartsch, G. Baumann, K. Stangl, K. Alexiou, Relaxin improves TNF-alpha-induced endothelial dysfunction: the role of glucocorticoid receptor and phosphatidylinositol 3-kinase signalling, Cardiovascular research 95 (1), 2012, 97-107, https://doi.org/10.1093/cvr/cvs149

[120] W.J. Cao, J.D. Rosenblat, N.C. Roth, M.A. Kuliszewski, P.N. Matkar, D. Rudenko, C. Liao, P.J. Lee, H. LeongPoi, Therapeutic Angiogenesis by Ultrasound-Mediated MicroRNA-126-3p Delivery, Arteriosclerosis, thrombosis, and vascular biology 35 (11), 2015, 2401-2411, https://doi.org/10.1161/ATVBAHA.115.306506

[121] R. Hinkel, D. Penzkofer, S. Zuhlke, A. Fischer, W. Husada, Q.F. Xu, E. Baloch, E. van Rooij, A.M. Zeiher, C. Kupatt, S. Dimmeler, Inhibition of microRNA-92a protects against ischemia/reperfusion injury in a large-animal model, Circulation 128 (10), 2013, 1066-1075, https://doi.org/10.1161/CIRCULATIONAHA.113.001904

[122] X. Yu, L. Deng, D. Wang, N. Li, X. Chen, X. Cheng, J. Yuan, X. Gao, M. Liao, M. Wang, Y. Liao, Mechanism of TNF-alpha autocrine effects in hypoxic cardiomyocytes: initiated by hypoxia inducible factor 1alpha, presented by exosomes, Journal of molecular and cellular cardiology 53 (6), 2012, 848-857, https://doi.org/10.1016/j.yjmcc.2012.10.002

[123] C. Bang, S. Batkai, S. Dangwal, S.K. Gupta, A. Foinquinos, A. Holzmann, A. Just, J. Remke, K. Zimmer, A. Zeug, E. Ponimaskin, A. Schmiedl, X. Yin, M. Mayr, R. Halder, A. Fischer, S. Engelhardt, Y. Wei, A. Schober, J. Fiedler, T. Thum, Cardiac fibroblast-derived microRNA passenger strand-enriched exosomes mediate cardiomyocyte hypertrophy, The Journal of clinical investigation 124 (5), 2014, 2136-2146, https://doi.org/10.1172/JCI70577

[124] B.W. van Balkom, O.G. de Jong, M. Smits, J. Brummelman, K. den Ouden, P.M. de Bree, M.A. van Eijndhoven, D.M. Pegtel, W. Stoorvogel, T. Wurdinger, M.C. Verhaar, Endothelial cells require miR-214 to secrete exosomes that suppress senescence and induce angiogenesis in human and mouse endothelial cells, Blood 121 (19), 2013, 3997-4006, S3991-3915, https://doi.org/10.1182/blood-2013-02-478925

[125] F. Jansen, X. Yang, M. Hoelscher, A. Cattelan, T. Schmitz, S. Proebsting, D. Wenzel, S. Vosen, B.S. Franklin, B.K. Fleischmann, G. Nickenig, N. Werner, Endothelial microparticle-mediated transfer of MicroRNA-126 promotes vascular endothelial cell repair via SPRED1 and is abrogated in glucose-damaged endothelial microparticles, Circulation 128 (18), 2013, 2026-2038, https://doi.org/10.1161/CIRCULATIONAHA.113.001720

[126] L. Barile, V. Lionetti, E. Cervio, M. Matteucci, M. Gherghiceanu, L.M. Popescu, T. Torre, F. Siclari, T. Moccetti, G. Vassalli, Extracellular vesicles from human cardiac progenitor cells inhibit cardiomyocyte apoptosis and improve cardiac function after myocardial infarction, Cardiovascular research 103 (4), 2014, 530-541, https://doi.org/10.1093/cvr/cvu167

[127] A. Fleury, M.C. Martinez, S. Le Lay, Extracellular vesicles as therapeutic tools in cardiovascular diseases, Frontiers in immunology 5 2014, 370, https://doi.org/10.3389/fimmu.2014.00370

[128] D. Mozaffarian, E.J. Benjamin, A.S. Go, D.K. Arnett, M.J. Blaha, M. Cushman, S.R. Das, S. de Ferranti, J.P. Despres, H.J. Fullerton, V.J. Howard, M.D. Huffman, C.R. Isasi, M.C. Jimenez, S.E. Judd, B.M. Kissela, J.H. Lichtman, L.D. Lisabeth, S. Liu, R.H. Mackey, D.J. Magid, D.K. McGuire, E.R. Mohler, 3rd, C.S. Moy, P. Muntner, M.E. Mussolino, K. Nasir, R.W. Neumar, G. Nichol, L. Palaniappan, D.K. Pandey, M.J. Reeves, C.J. Rodriguez, W. Rosamond, P.D. Sorlie, J. Stein, A. Towfighi, T.N. Turan, S.S. Virani, D. Woo, R.W. Yeh, M.B. Turner, C. American Heart Association Statistics, S. Stroke Statistics, Heart Disease and Stroke Statistics-2016 Update: A Report From the American Heart Association, Circulation 133 (4), 2016, e38-360, https://doi.org/10.1161/CIR.0000000000000350

[129] I. Banerjee, J.W. Fuseler, R.L. Price, T.K. Borg, T.A. Baudino, Determination of cell types and numbers during cardiac development in the neonatal and adult rat and mouse, American journal of physiology. Heart and circulatory physiology 293 (3), 2007, H1883-1891, https://doi.org/10.1152/ajpheart.00514.2007

[130] I. Coma-Canella, [Changes in myocardial function and perfusion after acute myocardial infarction], Revista espanola de cardiologia 56 (5), 2003, 433-435,

[131] S.F. Mohammed, D.T. Majure, M.M. Redfield, Zooming in on the Microvasculature in Heart Failure With Preserved Ejection Fraction, Circulation. Heart failure 9 (7), 2016, https://doi.org/10.1161/CIRCHEARTFAILURE.116.003272 


\title{
CHAPTER 3
}

\section{Neonatal apex resection triggers cardiomyocyte proliferation, neovascularization and functional recovery despite local fibrosis}

\begin{abstract}
Vasco Sampaio-Pinto ${ }^{1,2,3}$, Sílvia C. Rodrigues ${ }^{1,2}$, Tiago L. Laundos ${ }^{1,2,3}$, Elsa D. Silva ${ }^{1,2}$, Francisco Vasques-Nóvoa ${ }^{1,2,4}$, Ana C. Silva ${ }^{1,2,3,5}$, Rui J. Cerqueira ${ }^{4}$, Tatiana P. Resende ${ }^{1,2}$, Nicola Pianca ${ }^{6}$, Adelino Leite-Moreira ${ }^{4}$, Gabriele D'Uva ${ }^{6}$, Sólveig Thorsteinsdóttir ${ }^{7}$, Perpétua Pinto-do-Ó ${ }^{1,2,3, *}$ and Diana S. Nascimento ${ }^{1,2, *}$
\end{abstract}

${ }^{1}$ i3S - Instituto de Investigação e Inovação em Saúde, Universidade do Porto, Porto, Portugal;

2 INEB - Instituto Nacional de Engenharia Biomédica, Universidade do Porto, Porto, Portugal;

${ }^{3}$ ICBAS - Instituto de Ciências Biomédicas Abel Salazar, Universidade do Porto, Portugal;

${ }^{4}$ Departamento de Fisiologia e Cirurgia Cardiotorácica, Faculdade de Medicina da Universidade do Porto, Portugal;

${ }^{5}$ Gladstone Institutes, University of California San Francisco, San Francisco, USA;

${ }^{6}$ Scientific and Technological Pole, IRCCS MultiMedica, Milan, Italy;

${ }^{7}$ Departamento de Biologia Animal, cE3c - Centro de Ecologia, Evolução e Alterações Ambientais, Faculdade de Ciências, Universidade de Lisboa, Lisboa, Portugal.

* These authors contributed equally to this work.

Stem Cell Reports. 2018 Mar 13;10(3):860-874

doi: 10.1016/j.stemcr.2018.01.042 


\begin{abstract}
So far, opposing outcomes have been reported following neonatal apex resection in mice, questioning the validity of this injury model to investigate regenerative mechanisms. We performed a systematic evaluation, up to 180 days after surgery, of the pathophysiological events activated upon apex resection. In response to cardiac injury, we observed increased cardiomyocyte proliferation in remote and apex regions, neovascularization, and local fibrosis. In adulthood, resected hearts remain consistently shorter and display permanent fibrotic tissue deposition in the center of the resection plane, indicating limited apex regrowth. However, thickening of the left ventricle wall, explained by an upsurge in cardiomyocyte proliferation during the initial response to injury, compensated cardiomyocyte loss and supported normal systolic function. Thus, apex resection triggers both regenerative and reparative mechanisms, endorsing this injury model for studies aimed at promoting cardiomyocyte proliferation and/or downplaying fibrosis.
\end{abstract}




\section{Introduction}

Cardiovascular diseases are the leading cause of death worldwide (Mozaffarian et al., 2016), which is largely attributed to the limited regenerative capacity of the mammalian heart. However, this paradigm has been challenged by studies describing a developmental window for heart regeneration in mammals. Although the first reference to mammalian heart regeneration was provided by Mario Robledo in 1956 (Robledo, 1956), this concept only became robustly supported in 2011 after Sadek and colleagues demonstrated that murine hearts, when subjected to apex resection during the first day of life, were able to reestablish the myocardial tissue through activation of cardiomyocyte (CM) proliferation (Porrello et al., 2011). This report proposed that, like lower vertebrates (Flink, 2002; Poss et al., 2002), the mammalian heart holds an intrinsic ability to regenerate from injury, which could be exploited for therapeutic purposes. This work also set the stage for several studies identifying regulators of CM proliferation (Aurora et al., 2014; Bassat et al., 2017; D'Uva et al., 2015; Heallen et al., 2013; Mahmoud et al., 2013) or describing distinct injury models (Darehzereshki et al., 2015; Haubner et al., 2012; Jesty et al., 2012; Lavine et al., 2014; Porrello et al., 2013; Uygur and Lee, 2016).

In 2014, controversy arose when another laboratory, using the same injury model, reported substantial scarring in the resected apex region, which was accompanied by impaired neovascularization and CM proliferation (Andersen et al., 2014a). Further work by this group showed that fibrotic tissue deposition in the resected region was definitive, and that cardiac function was permanently reduced as animals developed dilated cardiomyopathy, with left ventricle (LV) chamber dilation and wall thinning (Andersen et al., 2016).

The reason for these contrasting findings is still unclear (Andersen et al., 2014b; Kotlikoff et al., 2014; Sadek et al., 2014), although technical variations such as injury severity were shown to impact the formation of scar tissue upon apex resection (Bryant et al., 2015) and cryoinjury (Darehzereshki et al., 2015). Thus, the ability of neonatal hearts to regenerate after apex resection seems to be dependent on small technical variations which at present hampers progress in the field.

Additional difficulties are related to the evaluation of the fibrotic scar (Andersen et al., 2014a; Bryant et al., 2015) and the fact that the assessment of CM proliferation, the most important hallmark for cardiac regeneration, is technically challenging. In particular, CM proliferation in mammals can be easily mistaken for $\mathrm{CM}$ binucleation that initiates around birth (Zebrowski et al., 2016), an event characterized by karyokinesis without cytokinesis, leading to CM terminal differentiation (Soonpaa et al., 1996). Of note, studies addressing the response to apex resection in neonatal mice have either focused on functional assessment or histopathological alterations (Andersen et al., 2014a), but longitudinal studies examining cardiac remodeling, function, and the precise tissue response to injury have not yet been performed.

Since there are antagonizing reports and because tissue regeneration and repair often activate overlapping mechanisms (Aurora et al., 2014; Tidball, 2011), we evaluated both tissue responses following neonatal apex resection. Hence, a systematic assessment of the 
pathophysiological events occurring over 180 days after surgery was performed. We found that apex resection triggers both regenerative (e.g. neovascularization and $\mathrm{CM}$ proliferation) and reparative (e.g. cardiac fibrosis) mechanisms which result in an adult heart with more $\mathrm{CMs}$, benign adaptive cardiac remodeling and restored systolic function.

\section{Results}

\section{Establishment of Neonatal Apex Resection Injury Model}

One-day-old (P1) C57BL/6 mice were subjected to apical resection by thoracotomy using the exposure of the LV lumen as a standardized method for resection size (Mahmoud et al., 2014; Porrello et al., 2011). Sham controls underwent the same surgical procedure without resection of the apex (no cardiac manipulation of any kind).

Immediately after the surgical procedure, the survival rate of apex-resected animals was equivalent to sham controls ( $\sim 87 \%)$. However, maternal cannibalization reduced survival at $24 \mathrm{~h}$ post-surgery to $61 \%$ and $82 \%$ for injury and sham groups, respectively.

\section{Hearts Are Not Fully Restored at the Histological Level}

The ventricular surface area and heart length/body weight ratio were significantly reduced upon apical resection by $\sim 11 \%$ and $\sim 14 \%$, respectively (Figures $1 \mathrm{~A}-1 \mathrm{C}$ ), similar to other reports (Andersen et al., 2014a; Porrello et al., 2011). Representative sampling of the heart (Figure S1A) and histological characterization by Masson's trichrome (MT) staining (Figures $1 \mathrm{~A}$ and $\mathrm{S} 1 \mathrm{~B}$ ) revealed that apex resection induced noticeable cardiac remodeling, with impact on heart morphology and local deposition of fibrotic tissue (Figures 1B-1D). From 7 to 21 days after surgery, apex regrowth was evident as myocardial tissue was observed below the resection site. Alongside, the myocardial tissue in the innermost part of the apex where the lumen was exposed, was surrounded by a subepicardial scar (Figures $1 \mathrm{~A}$ and S1B). Higher magnification revealed that the scar undergoes a maturation process, which starts with a scattered deposition of collagenous material and matures to a fibrillar appearance, occupying most of the central apex wall from 60 days after resection onward (Figures 1A1-1A4, S1B1, S1B2, and S1C). In contrast, areas surrounding the central apex region displayed myocardial integrity.

Ventricular surface area was restored at 7 days after injury (Figure 1B), and resected hearts weighed the same or were even heavier than intact hearts (Figures S1D and S1E). However, injured hearts were consistently shorter throughout the study, indicating that heart growth was mainly achieved through short-axis expansion (Figure 1C). The extent of injury, estimated by determining the percentage of sections with cardiac damage (i.e., myocardial disruption or cardiac fibrosis), decreased over time, which correlates with an actual reduction in scar volume (Figures $1 \mathrm{D}$ and $1 \mathrm{E}$ ). Overall, these results indicate that resected hearts remain shorter, have permanent scarring, and do not fully regenerate. 
A

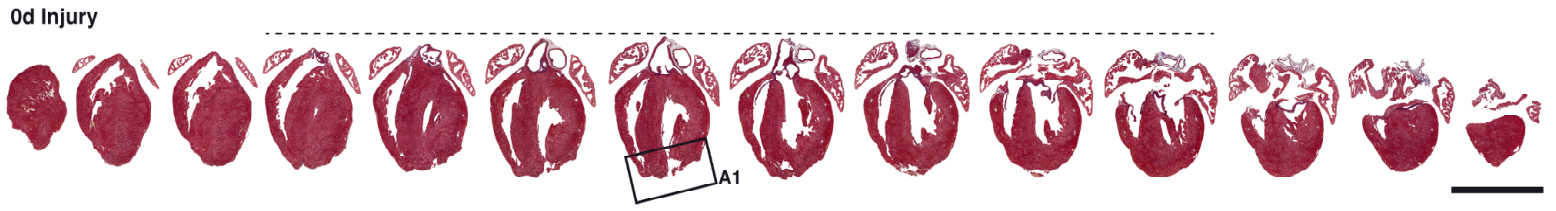

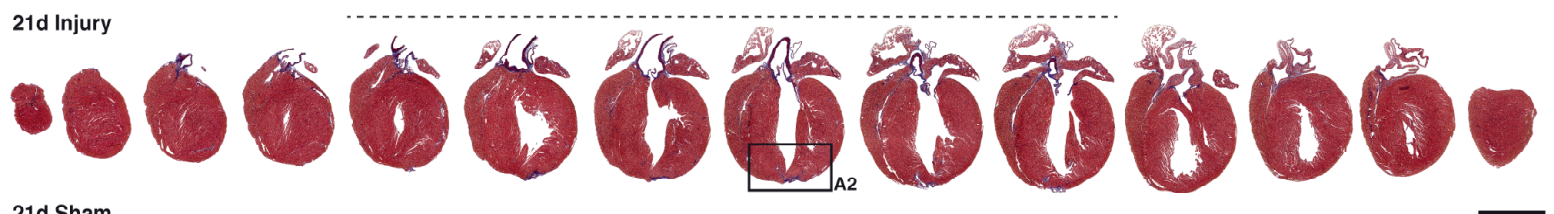

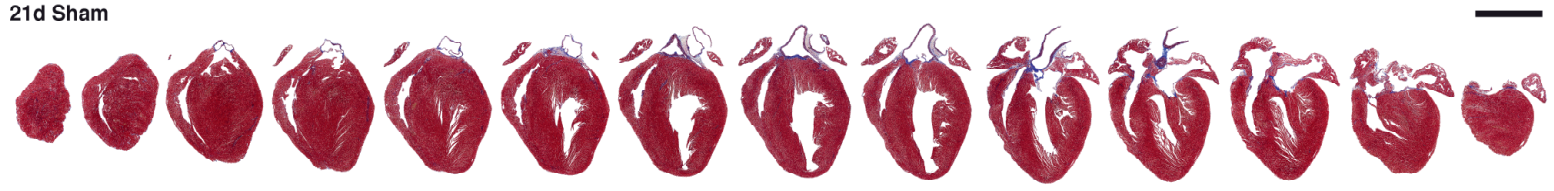

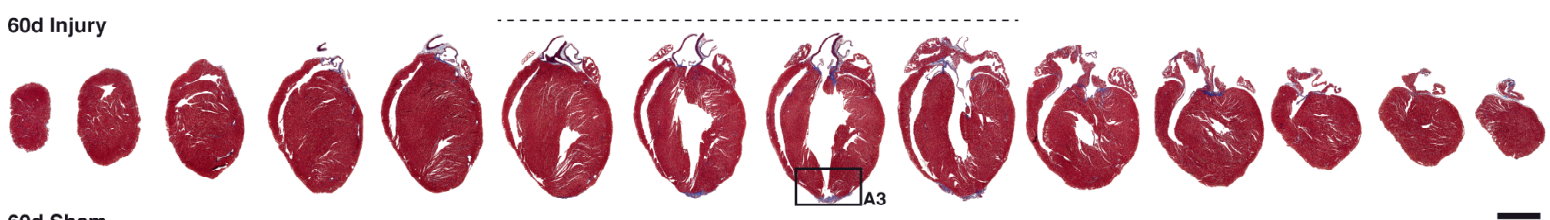

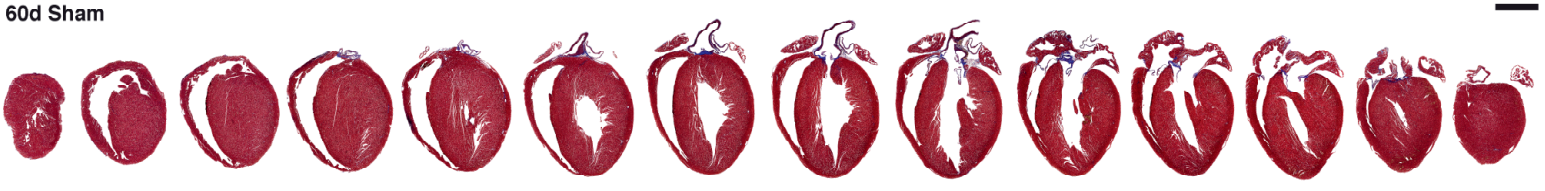
180d Injury

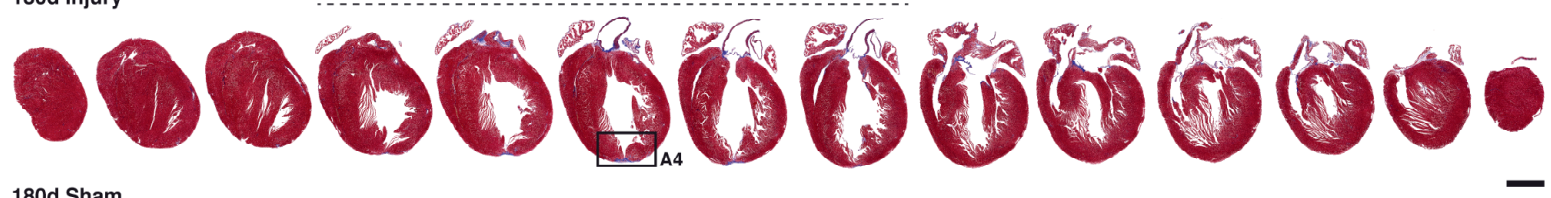

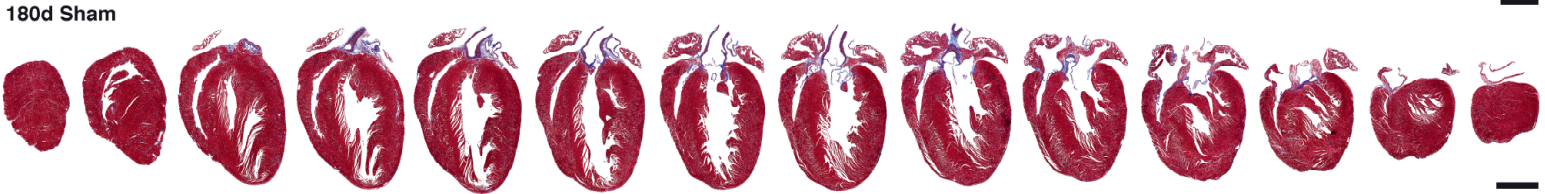
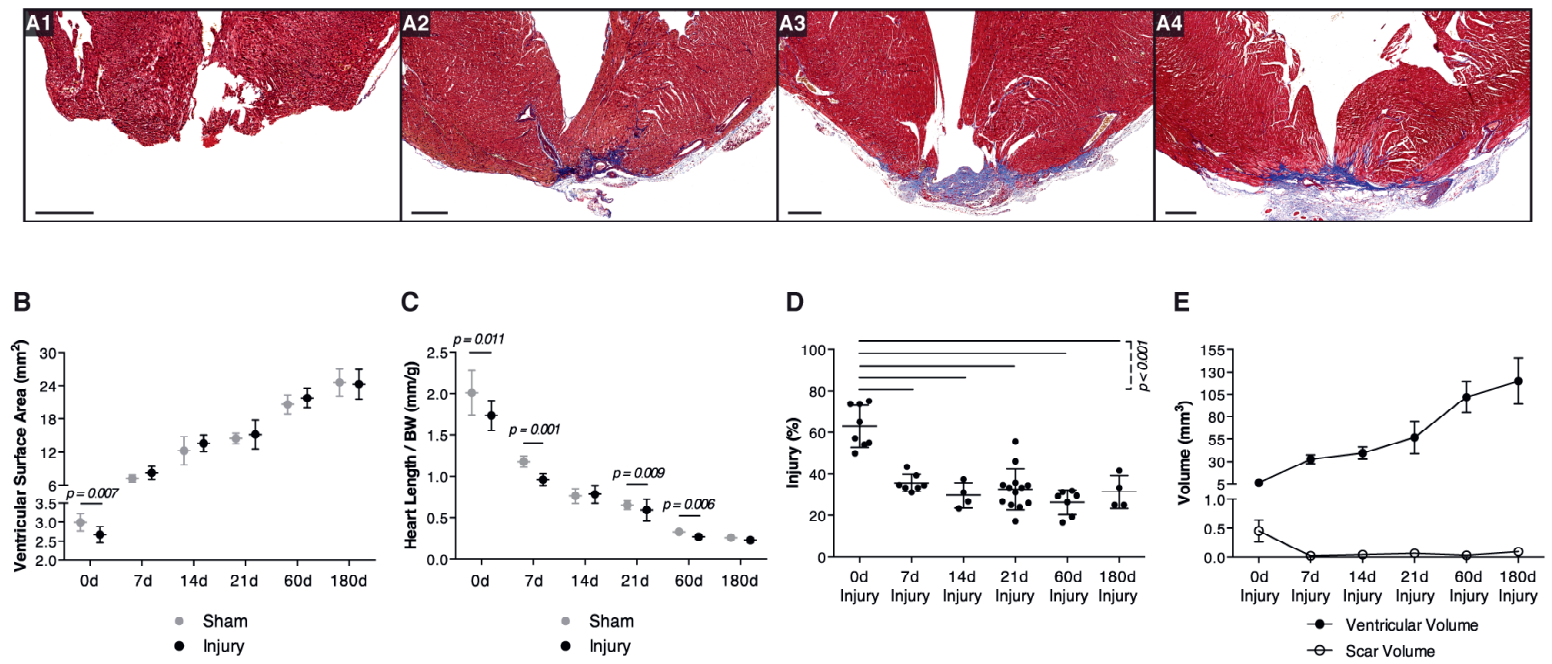

(legend on next page) 
Figure 1. Hearts Do Not Fully Regenerate the Resected Apex

(A) Representative MT-stained sections of apex-resected and sham controls following surgery. Sections exhibiting myocardial disruption and/or cardiac fibrosis are highlighted by a dashed line. Scale bars, $2 \mathrm{~mm}$. Highmagnification images of the injury site (A1, 0 day; A2, 21 days; A3, 60 days; and A4, 180 days) show collagen (blue staining) from 21 days onward. Scale bars, $250 \mu \mathrm{m}$.

( $B$ and C) Ventricular surface area (from left to right, $n=8,10,8,14,3,7,4,4$ ) (B) and heart length/ body weight (BW) (from left to right, $n=11,11,8,10,4,7,4,4)$ (C) of resected and sham-operated hearts were determined in MT-stained paraffin sections at $0,7,14,21,60$, and 180 days post-surgery.

( $D$ and $E$ ) Injury extension (from left to right, $n=8,12,7,4$ ) (D) and ventricular and scar volume (from left to right, $n=19,14,7,4)(E)$ were calculated on MT-stained paraffin sections. All values are presented as means \pm SD. See also Figure $\mathrm{S} 1$ for representative MT sections at 7 and 14 days after surgery, data on heart weight, heart to BW ratio, and percentage of fibrosis in the apex.

\section{Resected Hearts Are Functionally Competent}

Echocardiograms at $21,60,120$, and 180 days following surgery confirmed that resected ventricles underwent severe morphological alterations (i.e., long-axis shortening and shortaxis widening) (Figure 2A). However, in contrast to previous reports (Andersen et al., 2016), our data showed that short-axis expansion occurred due to a thickening of the anterior and posterior wall of the LV (Figures $2 \mathrm{~B}$ and $2 \mathrm{C}$ ) and left ventricular chamber dilation (Figure 2D). Because LV enlargement is concurrent to a reduction in heart length (Figure 2E), the eccentricity index of injured hearts was significantly increased (Figure 2F). End-diastolic volume of injured hearts was increased at 21 days, but normalized from 60 days onward (Figure 2G). Importantly, no differences were detected in the ejection fraction (Figure 2H), stroke volume (Figure 2I) or cardiac output (Figure 2J) across groups, thus showing that the systolic function of injured hearts was not significantly impaired. Regarding diastolic function, the ratio between LV early (E) to late (A) filling velocities was marginally decreased at 180 days, suggesting that incomplete relaxation and increased filling pressures might be present in resected hearts (Figure 2K). This can be explained by increased myocardial stiffness caused by the scarring, wall thickening, and/or alterations in ventricular chamber geometry. Accordingly, myocardial performance index (also known as Tei index), which comprises both systolic and diastolic parameters, showed a tendency to be increased in resected hearts (Figure $2 \mathrm{~L}$ ) at the expense of longer relaxation times (data not shown). Electrocardiogram (ECG) tracing of injured hearts was similar to control animals (Figure $2 \mathrm{M}$ ), which was confirmed by the absence of any significant modification in the evaluated ECG parameters (Table S1).

These findings demonstrate that, despite consistent LV chamber enlargement and wall thickening, accompanied by a reduced heart length, resected hearts are functionally competent. 
A

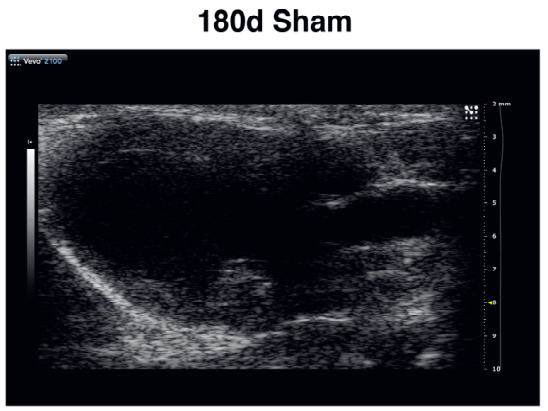

B

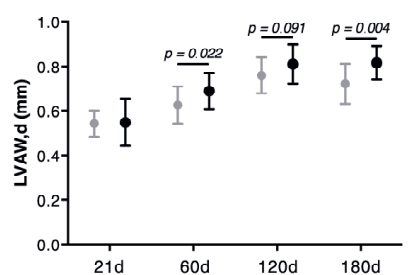

E

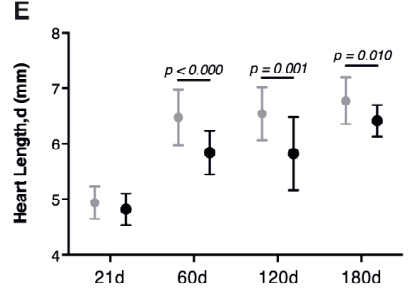

H

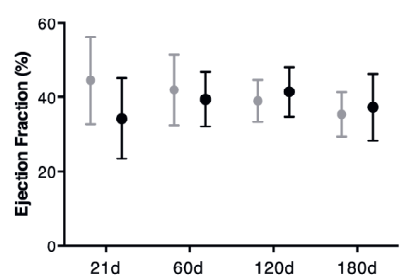

K

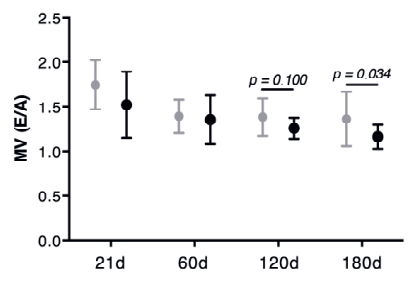

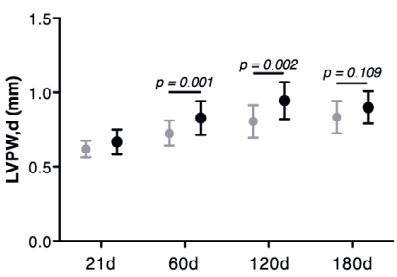

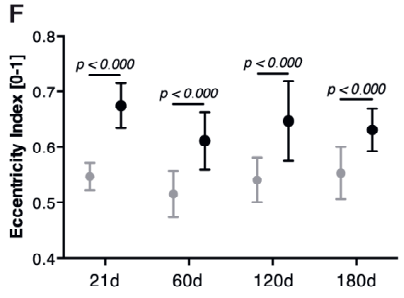

I

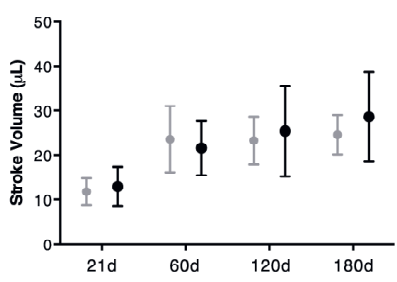

L

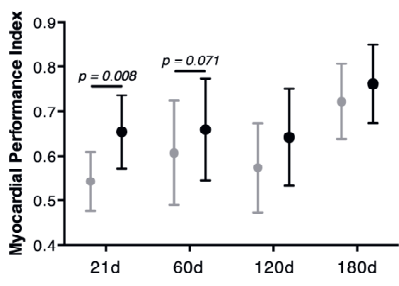

180d Injury

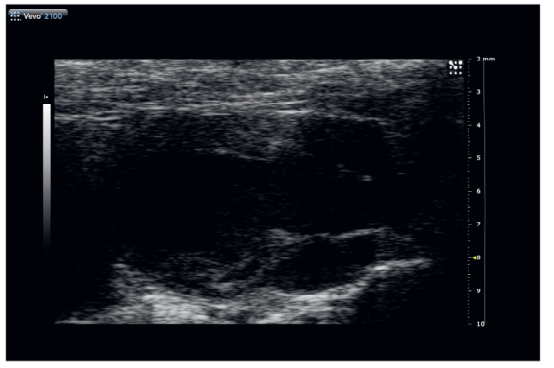

D

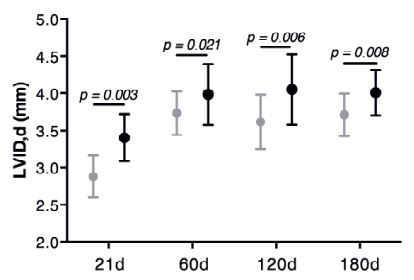

G
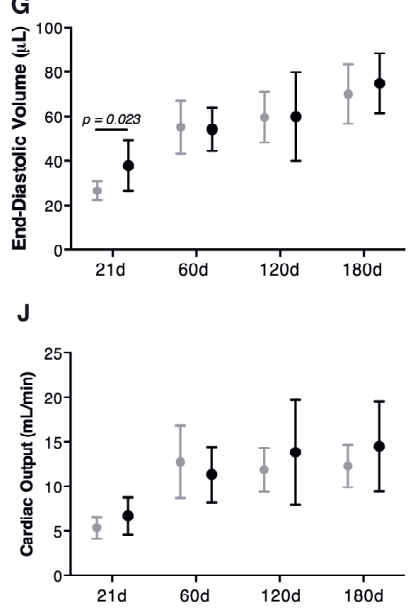

- Sham

- Injury

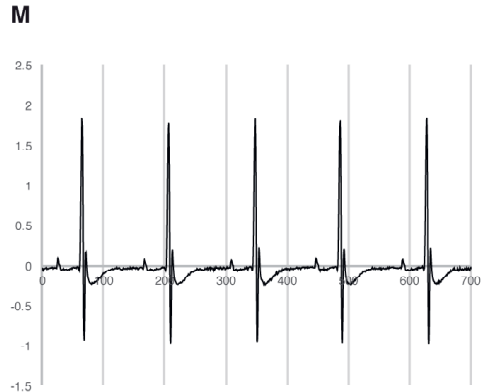

180d Sham

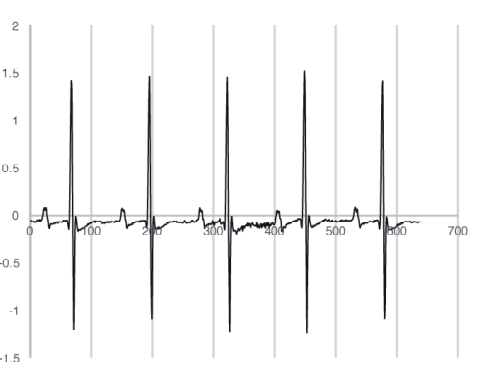

180d Injury

(legend on next page) 
Figure 2. Injured Hearts Display Morphological Alterations but Exhibit Normal Systolic Function

(A) Representative echocardiographic parasternal long-axis view 180 days post-surgery.

(B-G) End-diastolic left ventricle anterior wall thickness (LVAW), left ventricle posterior wall thickness (LVPW), left ventricle internal diameter (LVID), heart length, eccentricity index, and heart volume.

$(\mathrm{H}-\mathrm{J})$ Ejection fraction (EF), stroke volume and cardiac output determined by Simpson's method.

$(\mathrm{K}$ and $\mathrm{L}$ ) Ratio between left ventricular early $(\mathrm{E})$ to late $(\mathrm{A})$ filling velocities and myocardial performance index (also known as Tei index)

(from left to right, $n=7,9,22,24,18,15,18,14$ ).

(M) Representative ECG signal traces at 180 days post-surgery $(n=10,6 ; 180$ days sham, 180 days injury). See also Table S1 for detailed quantification of ECG parameters. All values are presented as means \pm SD.

Short-Term Response to Cardiac Injury Involves Extracellular Matrix Remodeling and Fibroblast Activation

Apex amputation was evident by the disruption of the expression pattern of sarcomeric- $\alpha$ actinin (s- $\alpha$-Actinin) and laminin (LN) (Figure S2). Forty-eight hours after resection, the apex region was marked by the deposition of fibronectin (FN) and tenascin-C (TN-C), and by an abundant $\mathrm{CD} 45^{+}$immune cell infiltrate (Figures $3 \mathrm{~A}$ and $\mathrm{S} 2$, arrowheads). Myofibroblasts ( $\alpha$ $\mathrm{SMA}^{+}$cells) also started to appear at the injury site at this point (Figure S2, arrows). Resolution of the inflammatory phase was evident at 7 days post-injury, whereas myofibroblasts became more frequent (Figures $3 \mathrm{~A}$ and S2). At 21 days post-resection, $\alpha-$ SMA was restricted to smooth muscle cells in vessels, as observed in sham hearts. Of note, TN-C remained elevated in the border between the resection and the adjacent myocardium (Figure S2). Confocal microscopy analysis further showed that FN and TN-C were produced by $\mathrm{CD}_{4} 5^{+}$hematopoietic cells during the inflammatory phase ( $48 \mathrm{~h}$ post-surgery) and by $\alpha$ $\mathrm{SMA}^{+}$myofibroblasts during inflammatory resolution (7 days post-surgery) (Figure $3 \mathrm{~A}$ ). Although largely overlapping, $\mathrm{TN}-\mathrm{C}$ expression was restricted to the interface between the healthy and damaged myocardium, whereas FN displayed a more ubiquitous deposition, suggesting different biological roles (Figure $3 \mathrm{~A}$ ).

We profiled hematopoietic cells $\left(C D 45^{+}\right)$infiltrating the apex and remote regions by flow cytometry at 2, 5, and 7 days after surgery. Single-cell suspensions were stained with antibodies to distinguish total myeloid cells $\left(C D 11 b^{+}\right)$, macrophages $\left(C D 11 b^{+} F 4 / 80^{+}\right)$, monocytes $\left(C D 11 b^{+} F 4 / 80^{-}\right)$, neutrophils $\left(C D 11 b^{+} G r-1^{+}\right)$, $T$ lymphocytes $\left(C D 3^{+}\right)$, and $B$ lymphocytes $\left(C D 45 R^{+}\right)$(Figure S3A). At 2 days after surgery, an influx of $\mathrm{CD}^{+} 5^{+}$cells was observed in the apex, paralleled by a reduction in the remote LV (Figures S3B and S3C). The rate of hematopoietic cells in the myocardium of apex-resected hearts was comparable with those observed in sham-operated controls at 7 days post-surgery, corroborating our immunofluorescence data. The immune infiltrate was mainly composed of myeloid cells. At the initial stages of injury-response (i.e., 2 days) neutrophils, monocytes, and macrophages infiltrated the myocardium at the apex, although only macrophages remained elevated up to 5 days post-surgery. No differences were observed regarding the percentage of $T$ lymphocytes between groups, whereas $B$ lymphocytes were significantly decreased 2 days post-injury (Figures S3B and S3C). Additional staining of myeloid populations allowed discrimination of $C D 11 b^{+} F 4 / 80^{+} L y 6 C^{10}$ macrophages, $C D 11 b^{+} F 4 / 80^{+} L y 6 C^{h i}$ macrophages, 
CD $11 b^{+} F 4 / 80^{-}$Ly6C $^{10}$ monocytes, and CD11 ${ }^{+} F 4 / 80^{-}{\text {Ly } 6 C^{\text {hi }}}^{-}$inflammatory monocytes, as reported previously (Aurora et al., 2014). Of note, CD11 b ${ }^{+} \mathrm{F} 4 / 80^{+} \mathrm{Ly} 6 \mathrm{C}^{\mathrm{hi}}$ macrophages and $\mathrm{CD} 11 \mathrm{~b}^{+} \mathrm{F} 4 / 80^{-}{ }^{-} \mathrm{L} 6 \mathrm{C}^{\text {hi }}$ monocytes were predominantly increased at the injury site, but not in the remote myocardium, during early inflammatory response (Figure S3D). In turn, $\mathrm{CD} 11 \mathrm{~b}^{+} \mathrm{F} 4 / 80^{+} \mathrm{Ly} 6 \mathrm{C}^{\mathrm{lo}}$ macrophages were increased at 5 days after injury, similar to what was reported following myocardial infarction at P1 (Aurora et al., 2014).

Cardiac fibroblasts are master mediators of myocardial remodeling following injury (reviewed in (Souders et al., 2009)). However, studies determining the cardiac fibroblast surface signature during the neonatal period are lacking. Considering the heterogeneity of this cell type, we discriminated fibroblast populations, from embryonic day 17 to adulthood (Figure S4), through the expression of the three well-reported markers of cardiac fibroblasts, i.e., CD90 (Thy-1), CD140a (PDGFRa), and SCA-1 (Furtado et al., 2014; HudonDavid et al., 2007; McQualter et al., 2009; Smith et al., 2011), following exclusion of nonviable cells $\left(\mathrm{PI}^{+}\right)$, endothelial cells $\left(\mathrm{CD} 31^{+}\right)$, hematopoietic cells $\left(\mathrm{CD} 45^{+}\right)$, and erythrocytes $\left(\right.$ TER-119 ${ }^{+}$. These results demonstrated that fibroblasts express the mesenchymal marker CD140a ( $\geq 85 \%$ ) at all stages analyzed, either in the presence or absence of CD90 (Figure S4). Throughout time these fibroblasts also acquire the expression of SCA- 1 and, thus, in the adult, the most abundant subsets are CD140a ${ }^{+} \mathrm{CD} 90-\mathrm{SCA}-1^{+}$(Figure S4, green) and CD140a ${ }^{+}$CD90+SCA-1+ (Figure S5, gray), as reported previously (Furtado et al., 2014).

We applied the same strategy to injured and sham-operated animals 7 days post-surgery (Figure 3B). Three main populations were identified: CD140a+CD90-SCA-1- (blue; injury: $34.71 \% \pm 2.24 \%$; sham: $32.50 \% \pm 2.72 \%$ ), $C D 140 a^{+}{ }^{+}$CD90 ${ }^{+} S C A-1^{-}$(purple; injury: $36.53 \% \pm$ 0.95\%; sham: $40.29 \% \pm 1.85 \%$ ), and $\mathrm{CD} 140 \mathrm{a}^{+} \mathrm{CD} 90^{+} \mathrm{SCA}-1^{+}$(gray; injury: $21.26 \% \pm 1.15 \%$; sham: $17.77 \% \pm 1.40 \%$ ). A minor population was also found expressing CD90 only (red; injury: $3.79 \% \pm 0.94 \%$; sham: $6.34 \% \pm 1.17 \%$ ), whereas SCA-1 was mainly confined to cells also expressing the other surface markers (Figure 3B). No statistically significant differences were detected in the relative percentages of each population between injured animals and sham-operated controls (Figure 3B). This might be explained by the use of the whole heart and/or by variability created during the isolation procedure, which may mask small differences in cell numbers in the apex. Indeed, immunofluorescence results showed a fibroblast-mediated response to injury largely confined to the lesion site (Figures $3 \mathrm{~A}$ and S2).

The activation of most abundant populations was assessed by real-time qPCR (sorting strategy detailed in Figure S5). The $\mathrm{CD} 140 \mathrm{a}^{+} \mathrm{CD} 90^{+} \mathrm{SCA}-1^{-}$(purple, SP1) subpopulation, which is particularly frequent in earlier developmental periods (Figure S4), responded to apex resection by upregulating genes associated with scarring (Col1a1, Col3a1, Tgfb1, and Tgfb3), CM proliferation ( $F n 1, T b \times 20, \lg f 1, \lg f 2$, and $F s t / 1)$, and neovascularization (Vegfa) (Figure $3 C$ ). In contrast, $\mathrm{CD} 90^{+} \mathrm{CD} 140 \mathrm{a}^{+} \mathrm{SCA}-1^{+}$(gray, SP3) cells, a subpopulation upsurging after birth (Figure $\mathrm{S4}$ ), shows a repair-associated phenotype, with increased expression of Col1a1, Col3a1, Tgfb1, and Tgfb3 (Figure 3C). CD140a ${ }^{+}$CD90-SCA-1- (blue, SP2) cells, more frequent in earlier developmental periods (Figure S4), were not responsive to injury, although they exhibited high expression levels of most evaluated transcripts (Figure $3 \mathrm{C}$ ). 
These findings indicate that development-associated fibroblasts support the regenerative response, whereas fibroblast subsets abundant in adulthood signal mostly toward scar formation.
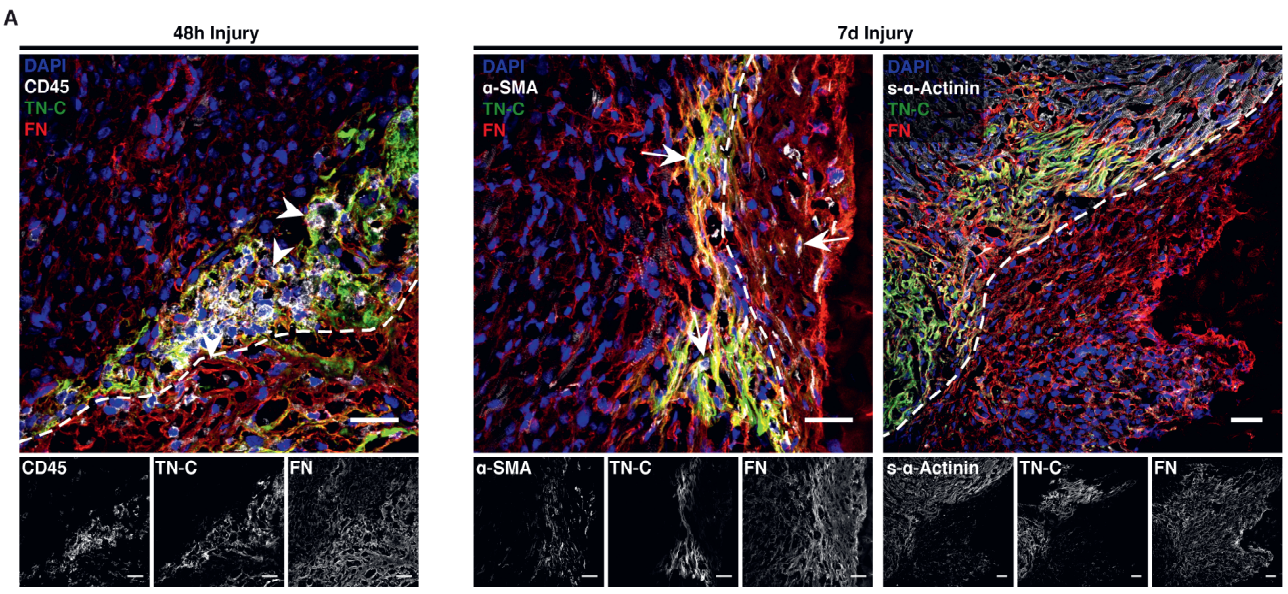

B

7d Sham

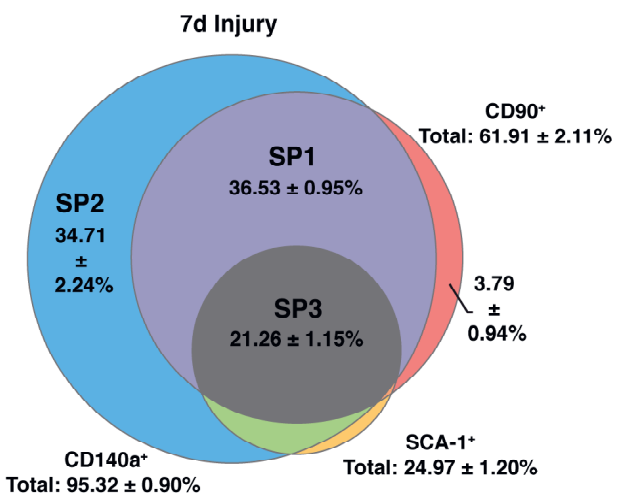

Total: $92.96 \pm 2.92 \%$

$20.83 \pm 1.87 \%$

CD140a $a^{+}$

Total: $95.32 \pm 0.90 \%$
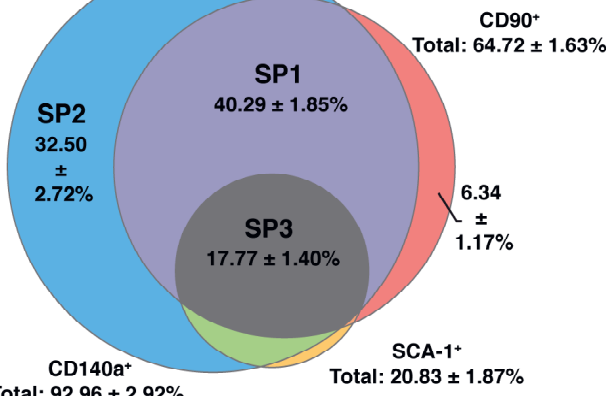

$\mathrm{CD} 90^{+}$

(C) SCA-1+
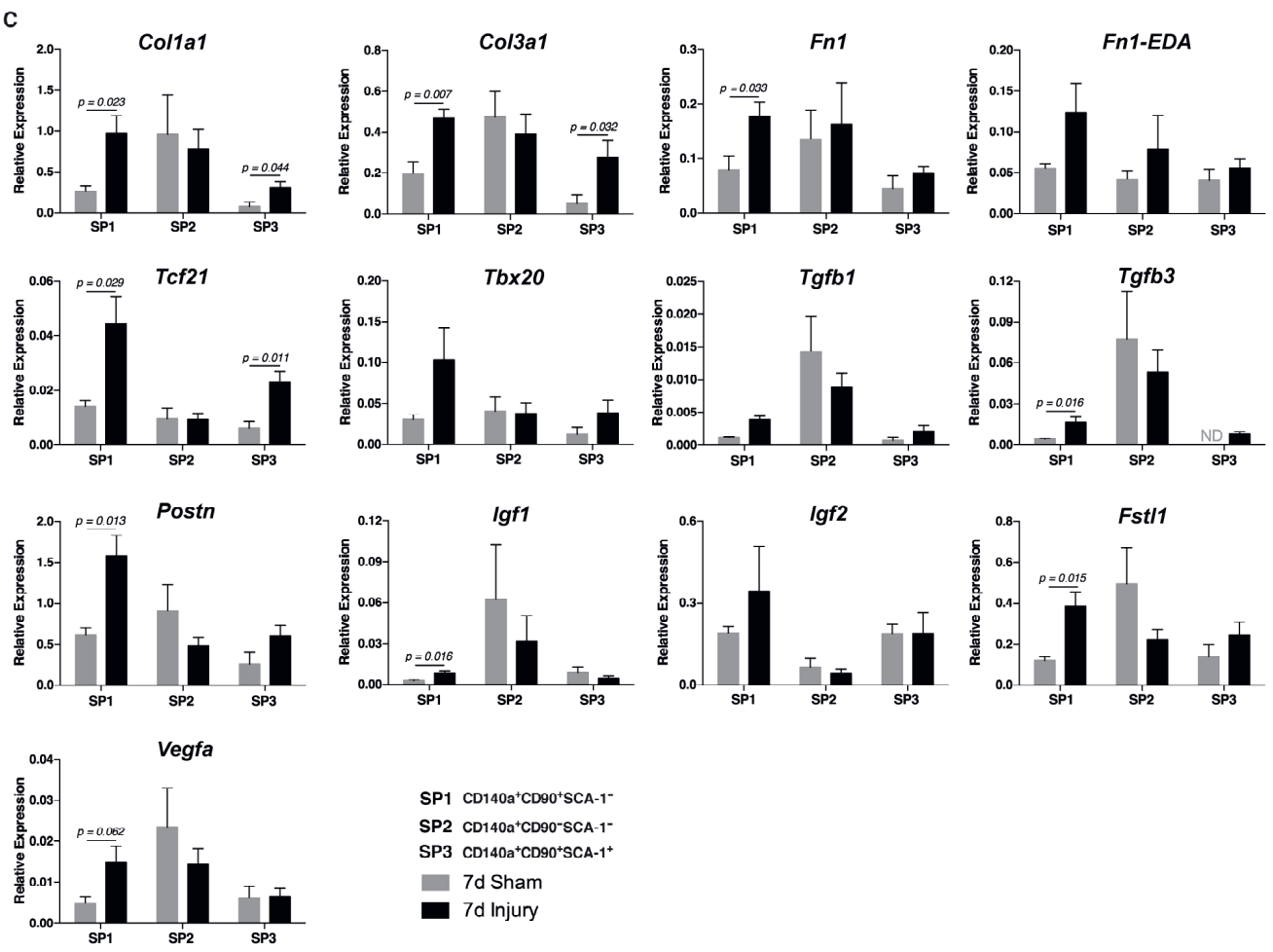

SP1 CD140a ${ }^{+}$CD90+SCA-

SP2 $\mathrm{CD}^{2} 40 \mathrm{a}^{\mathrm{C}} \mathrm{CD} 90^{-} \mathrm{SCA}-1$

SP3 $\mathrm{CD} 140 \mathrm{a}^{+} \mathrm{CD} 90^{+} \mathrm{SCA}-1^{+}$

7d Sham

- 7d Injury

(legend on next page) 
Figure 3. Apex Resection Triggers Local ECM Remodeling and Fibroblast Activation

(A) Representative confocal images of CD45/TN-C/FN, $\alpha$-SMA/TN-C/FN, and s- $\alpha$-Actinin/TN-C/FN immunolabelling at the injury site $48 \mathrm{~h}(n=4)$ and 7 days post-surgery $(n=4)$. Arrowhead, $C D 45^{+}$hematopoietic cells; arrows, $\alpha$ $\mathrm{SMA}^{+}$myofibroblasts; dashed segment, resection line. Scale bars, $30 \mu \mathrm{m}$. See also Figure $\mathrm{S} 2$ for detailed analysis of cellular and extracellular matrix (ECM) remodeling following injury.

(B) Cardiac fibroblasts were isolated and prospectively identified by CD140a, CD90, and SCA-1 expression throughout ontogeny (see Figure $S 4), 7$ days post-resection $(n=3)$ and sham surgery $(n=6) . C D 140 a^{+} C D 90^{+} S C A-1^{-}$ (purple/SP1), CD140a+CD90-SCA-1- (blue/SP2), and CD140a ${ }^{+} C D 90^{+} S C A-1^{+}($gray/SP3) fibroblasts were FACS-sorted (see Figure $\mathrm{S} 5$ for sorting strategy).

(C) Transcriptomic profiling of SP1 to SP3 subpopulations isolated from injured $(n=5)$ and sham hearts $(n=4)$ was performed by real-time qPCR. Expression levels were normalized against Gapdh. All values are presented as means \pm SEM. See also Figures S4 and S5 for detailed phenotypic characterization of cardiac fibroblasts throughout ontogeny and sorting strategy, respectively.

\section{Proliferation Is Induced upon Cardiac Injury and Leads to Newly Formed CMs}

The activation of $\mathrm{CM}$ proliferation after apex resection has been a matter of intense debate (Zebrowski et al., 2016). At 7 days post-injury, immunostaining for histone H3 phosphorylated at serine 10 (PH3) and s- $\alpha$-Actinin highlighted cycling $\mathrm{CMs}$ that were distributed throughout the myocardium. Quantification of $\mathrm{PH}^{+} \mathrm{CMs}$ in the myocardium below the papillary muscle and in isolated CMs from the same region showed that injured hearts displayed increased rates of mitotic $\mathrm{CMs}\left(\mathrm{PH}^{+}\right)$in comparison with sham-operated hearts (Figures $4 \mathrm{~A}$ and $4 \mathrm{~B}$ ). Moreover, CMs undergoing mitosis show sarcomere disruption, which is essential for effective cell division (Figures 4 C and S6A; Movies S1, S2, and S3). These findings were further supported by a significant increase in CMs displaying Aurora $B$ kinase in the nucleus and in the cleavage furrow, of the border zone and remote myocardium, of resected hearts, indicating efficient cytokinesis (Figures $4 \mathrm{D}$ and $4 \mathrm{E}$ ). Close to the injury, mitotic $\mathrm{CMs}$ were found predominantly near $\mathrm{FN}$, but not TN-C. In accordance, $\mathrm{CD} 29$ ( $\beta 1$ integrin), which forms an integral part of several integrins involved in cell-matrix interactions, was found in most CMs (leda et al., 2009) (Figure S6).

The loss of CM proliferative potential is commonly attributed to the progression to a terminally differentiated phenotype characterized by the presence of a mature sarcomeric apparatus and binucleation (Collesi et al., 2008). To assess whether increased rates of mitosis correlated with higher rates of $\mathrm{CM}$ proliferation rather than multinucleation, we assessed the number of nuclei and morphometric parameters of CMs isolated from the apex by imaging flow cytometry. Injured hearts showed less multinucleation and a significant increase of mononucleated and round-shaped CMs, attesting successful cellcycle progression and cell division (Figures $4 \mathrm{~F}$ and $4 \mathrm{G}$ ).

Next, we applied stereology to establish whether the proliferative upsurge observed upon apex resection impacts on total $\mathrm{CM}$ numbers. The mice were treated with the thymidine analog 5-ethynyl-2'-deoxyuridine (EdU), and the frequency of EdU incorporation in thick tissue sections was determined by co-labeling with PCM-1, a marker of CM nuclei (Figure $4 \mathrm{H})$. The number of $\mathrm{EdU}^{+} \mathrm{CMs}$ was greater in resected hearts relative to sham-operated controls (Figures $4 \mathrm{H}-4 \mathrm{~J}$ ), further supporting an increment in $\mathrm{CM}$ proliferation after injury. 
Although CM proliferation was observed throughout the $\mathrm{LV}$, the frequency of $\mathrm{EdU}^{+} \mathrm{CMs}$ was more pronounced in regions proximal to the apex, compared with remote regions (Figure $4 \mathrm{~J})$. In accordance, the density of myocyte nuclei was found to be significantly increased in the apex regions (Figure 4K). Combination of collagen type IV (COLL IV), to delineate cell boundaries, with PCM-1 immunolabelling, showed that CM binucleation in injured $195.7 \%$ $\pm 0.93 \%)$ and sham-operated hearts $(95.98 \% \pm 0.86 \%)$ was similar at 14 days after surgery (Figure 4L).

Taking multinucleation into account, we determined the density of CMs in the tissue. The volume of the LV was calculated based on morphometric analysis of longitudinal tissue sections (Figure S1A) and corrected for tissue shrinkage during fixation and processing for paraffin embedding. The total number of LV CMs in resected hearts, calculated based on $\mathrm{CM}$ density in the remote region, was significantly higher relative to sham-operated controls (Figure 4M). These findings show that the injury-activated CM proliferation burst, although more abundant in the apex, is also extended to remote regions, overcompensating the amount of $\mathrm{CM}$ loss after resection.

Resected Hearts Display Increased Capillary Density and No Signs of CM Hypertrophy or Edema

To evaluate the status of the capillary network, endothelial cells $\left(\mathrm{CD} 31^{+}\right)$were quantified 60 days after surgery. The number of $\mathrm{CD} 31^{+}$endothelial cells was significantly increased in injured hearts in comparison with sham-operated animals both in the apex and in the remote myocardium (Figures $5 \mathrm{~A}$ and $5 \mathrm{~B}$ ).

To further establish whether hypertrophy was also contributing for LV wall expansion, CM size was assessed in tissue sections and isolated CMs. No significant alterations were detected in the cross-sectional area of CMs over time and in different ventricular regions, apart from the vicinity of fibrotic tissue, where CMs had a larger cross-sectional area at 180 days post-surgery (Figures $5 \mathrm{C}$ and $5 \mathrm{D}$ ). Corroborating these findings, no significant differences were found in the area and volume of isolated CMs, suggesting that this hypertrophic behavior is residual and mainly localized in the apex (Figures $5 \mathrm{E}-5 \mathrm{H}$ ). Furthermore, as edema could also explain the increase in myocardial volume, through the accumulation of fluid in the extracellular interstitial space, we examined the myocardial water content at 180 days post-surgery, but no differences were detected between the two groups (Figure 5I). Overall, these results further support that the LV wall thickening in resected hearts results from an increase in the number of CMs and is thus a consequence of injury-activated proliferation. 
Neonatal apex resection triggers cardiomyocyte proliferation, neovascularization and functional recovery despite local fibrosis
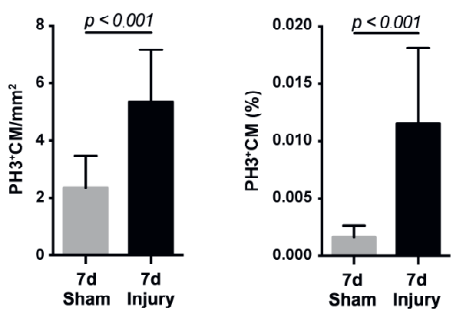

D

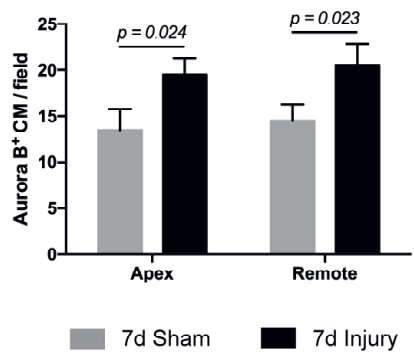

F

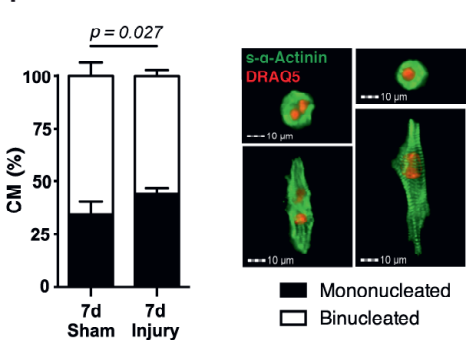

H
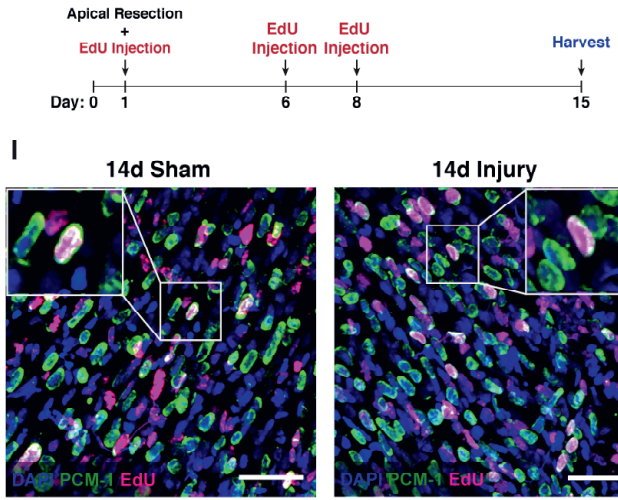

K

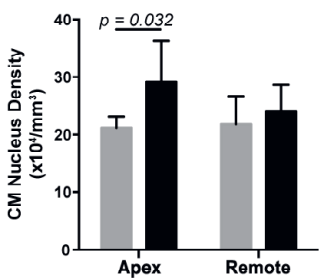

- $14 \mathrm{~d}$ Sham

- 14d Injury

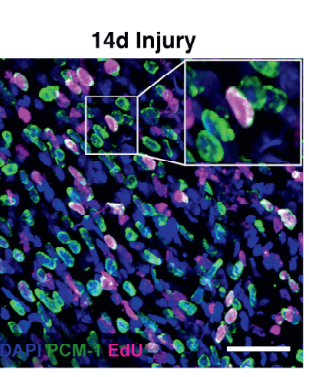

L

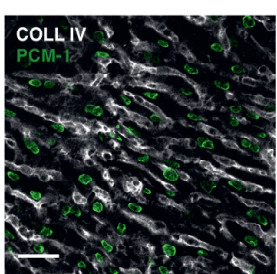

C

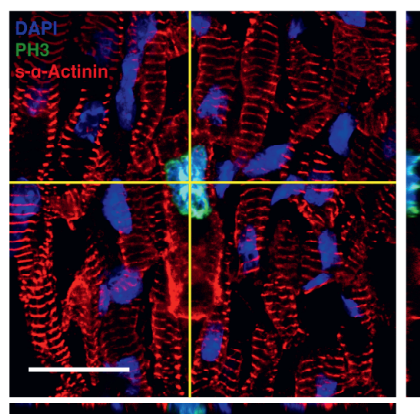

E

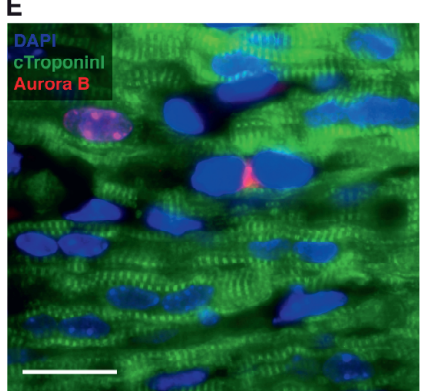

G

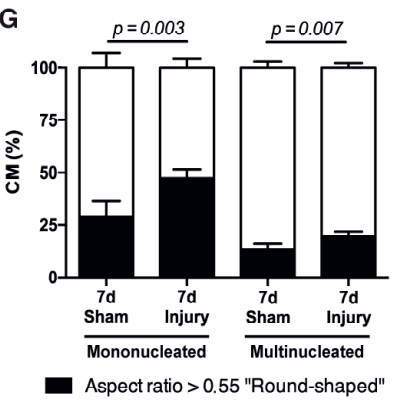

$\square$ Aspect ratio < 0.55 "Rod-shaped"

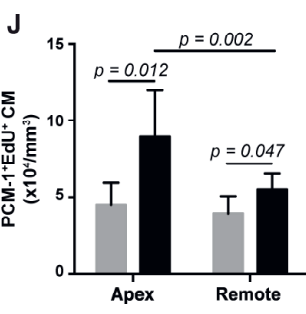

14d Sham

M

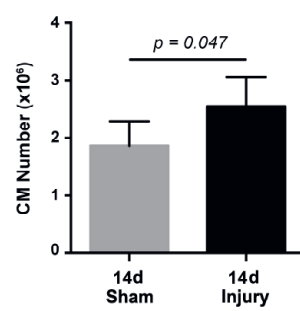

(legend on next page) 
Figure 4. Activation of CM Proliferation and Not Binucleation Generates a Heart with Increased CM Number upon Apex Resection

(A and B) Quantification of PH3 expression by CMs in the area between papillary muscles and the apex (A) $(n=10$, 10; 7 days sham, 7 days injury) and of isolated CMs from the same region (B) $(n=12,11 ; 7$ days sham, 7 days injury).

(C) Representative confocal image and respective orthogonal views of a CM expressing PH3 and clearly displaying sarcomere disassembly. Scale bar, $20 \mu \mathrm{m}$. See also Movies S1, S2, and S3.

(D) Quantification of Aurora B expression in CMs in regions close to the apex and remote myocardium $(n=3,3 ; 7$ days sham, 7 days injury).

(E) Representative image of Aurora B expression in proliferating CMs at 7 days post-surgery $(n=3)$. Scale bar, 20 $\mu \mathrm{m}$.

(F) Assessment of CM nucleation in the apex region at 7 days post-surgery and representative images $(n=5,4 ; 7$ days sham, 7 days injury).

(G) Ratio between round-shaped (immature) and rod-shaped (mature) CMs in mononucleated and multinucleated subpopulations of CMs isolated from the apex ( $n=6,5 ; 7$ days sham, 7 days injury).

(H) Three EdU pulses (days 1, 6, and 8) were given to trace proliferating CMs. Hearts were harvested 14 days postsurgery.

(I) Representative confocal images of PCM-1 $1^{+} \mathrm{EdU}+\mathrm{CM} 14$ days post-surgery. Scale bars, $40 \mu \mathrm{m}$.

(J) Quantification of EdU incorporation in PCM-1+ CMs in the apical and remote myocardium ( $n=4,7 ; 14$ days sham, 14 days injury).

(K) CM nucleus density at 14 days post-surgery ( $n=4,7 ; 14$ days sham, 14 days injury).

(L) Representative confocal image of COLL IV expression in the myocardium, which allowed the delineation of CM cell boundaries and nucleation evaluation ( $n=4,7 ; 14$ days sham, 14 days injury). Scale bar, $30 \mu \mathrm{m}$.

(M) Stereological estimate of the total number of CMs in the myocardium ( $n=4,7 ; 14$ days sham, 14 days injury). All values are presented as means \pm SD.

\section{Discussion}

Sadek and colleagues reported that apex-resected murine hearts at P1 displayed regenerative capacity (Porrello et al., 2011). Others have thereafter described a lack of histological restoration, with formation of a fibrotic scar, ultimately leading to LV dilation and reduced systolic function (Andersen et al., 2014a; Andersen et al., 2016). We performed a detailed evaluation of cellular and histo-functional alterations up to 180 days after resection and showed a well-orchestrated response in which a combined action of regenerative and reparative mechanisms is triggered, culminating in myocardial growth alongside fibrosis deposition in the innermost part of the apex. Therefore, our results are neither compatible with full regeneration (Porrello et al., 2011) nor exclusively with repair (Andersen et al., 2014a; Andersen et al., 2016), rather showing an intermediate response which likely explains the opposing reports in the field.

As previously debated, the methodology employed during surgery and in the assessment of the extent of regeneration is determinant for obtaining reliable results and outcomes (Andersen et al., 2014b; Sadek et al., 2014). Similar to other authors (Mahmoud et al., 2014; Porrello et al., 2011), we used LV cavity exposure as a standardized method for the resection. The resultant injury, assessed by measuring the ventricular surface area, as in Porrello et al., was $\sim 11 \%$ of the LV and therefore less severe than the $15 \%$ reported in their study. 
A

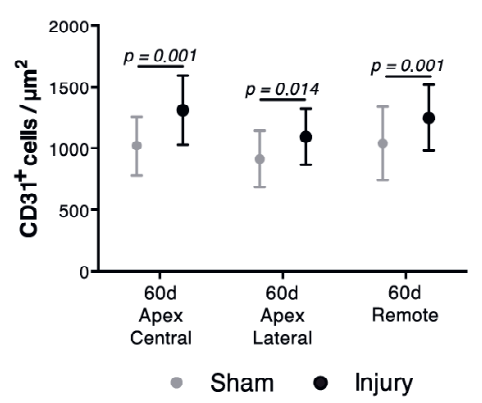

C

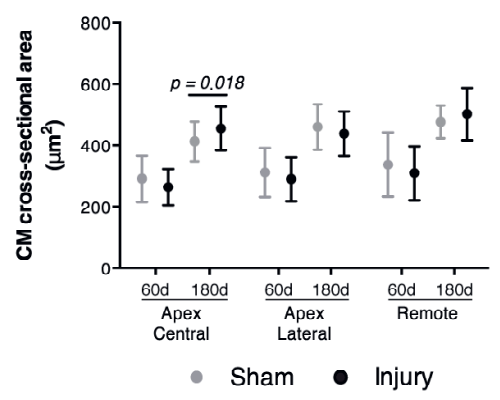

E

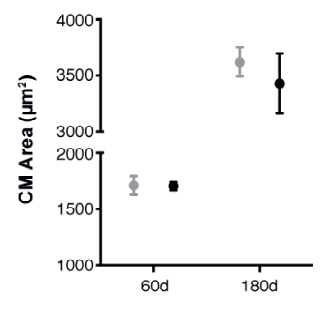

- Sham • Injury

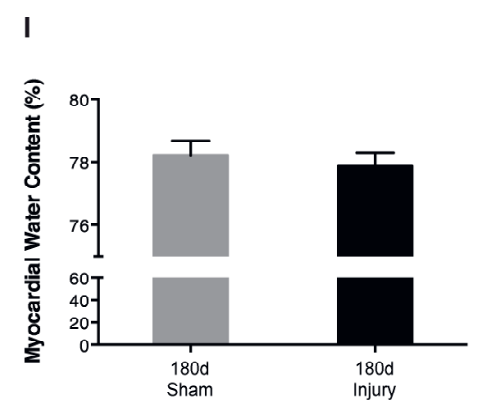

60d Sham
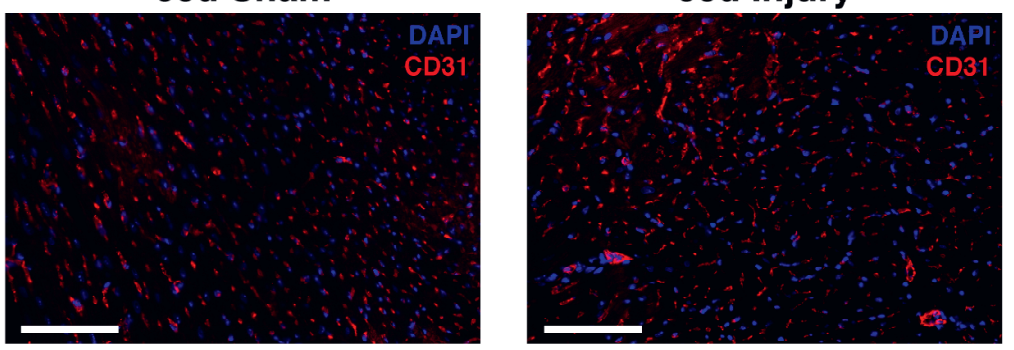

D

180d Sham
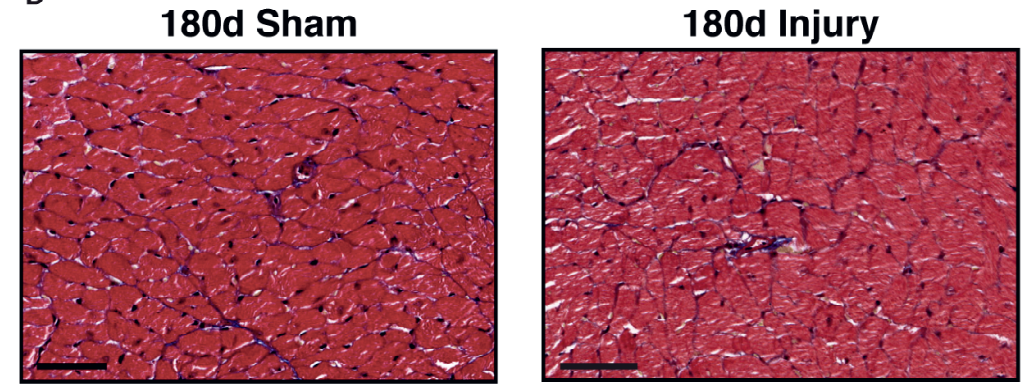

G

H
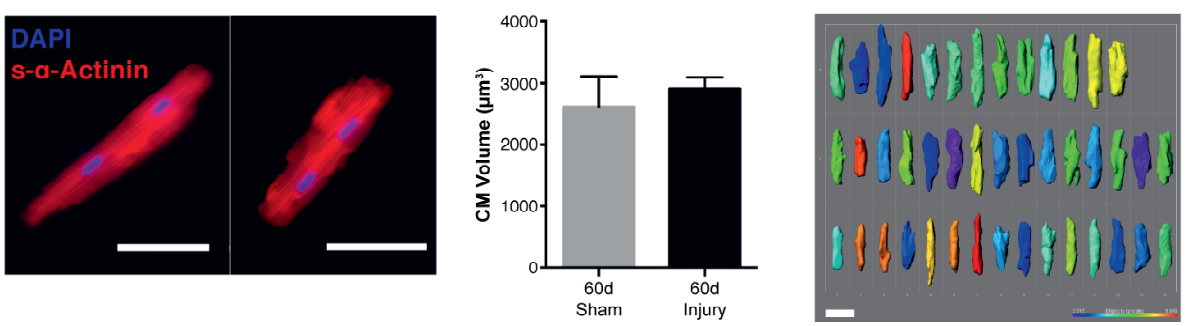

Figure 5. Resected Hearts Display Increased Coronary Vasculature but Not Hypertrophy or Edema

( $A$ and $B$ ) Capillary density at 60 days post-surgery (i.e., number of $C D 31^{+}$endothelial cells per unit area) $(n=6,7$; 60 days sham, 60 days injury). Scale bars, $10 \mu \mathrm{m}$.

(C and D) CM cross-sectional area at 60 and 180 days post-surgery $(n=6,6,4,4 ; 60$ days sham, 60 days injury, 180 days sham, 180 days injury). Scale bars, $25 \mu \mathrm{m}$.

(E and F) Area of isolated CMs at 60 and 180 days post-surgery $(n=3,5,4,4 ; 60$ days sham, 60 days injury, 180 days sham, 180 days injury). Scale bar, $100 \mu \mathrm{m}$.

(G) Volume of isolated CMs at 60 days post-surgery ( $n=4,6$ sham, injury).

(H) Representative images of 3D segmentation of CMs. Scale bars, $50 \mu \mathrm{m}$.

(I) Myocardial edema was assessed by myocardial water content quantification (i.e., percentage of myocardial weight that is lost after desiccation) 180 days post-surgery ( $n=10,6 ; 180$ days sham, 180 days injury). All values are presented as means \pm SD. 
By quantifying (1) the incidence of sarcomere-disassembly associated with PH3, (2) EdU $\mathrm{CMs}$ in a pulse-chase experiment, and (3) Aurora B kinase in the nuclei and cleavage furrow of CMs, we showed that $\mathrm{CMs}$ of resected hearts exhibit increased mitotic rates, accompanied by cytokinesis. During the first week of post-natal life in mice the majority of CMs binucleate, exit from the cell cycle, and continue to grow in size (Soonpaa et al., 1996). Several lines of evidence support that proliferating $\mathrm{CMs}$ are mononucleated and that binucleation marks an end to cell-cycle activity during development (Bersell et al., 2009; Paradis et al., 2014). By analyzing isolated CMs by imaging flow cytometry we found that, 7 days following apex resection, CM binucleation is reduced and accompanied by an increase in the pool of mononucleated round-shaped CMs, further supporting that, in response to injury, CMs undergo karyokinesis and cytokinesis. At 14 days post-resection the levels of binucleation were already comparable with sham-operated animals, meaning that newly formed CMs follow a normal maturation progression. These results contrast with a decrease in CM mitotic activity after injury observed by Andersen et al., in which a single EdU injection was administered at the onset of surgery, most likely missing the CM proliferative burst. In a recent publication, apex resection was shown to promote a transient acceleration in binucleation, an event no longer noticeable at P8 (Zebrowski et al., 2017). According to this study, a lower number of CMs would be expected in hearts subjected to apex amputation, which was not addressed by the authors. Herein, total CM numbers, as estimated by stereology, were increased in resected hearts compared with sham controls, indicating that the majority of injury-induced CM cell-cycle activity results in proliferation, not binucleation. In the work by Zebrowski and colleagues, isolated CMs were identified by sarcomeres in phase contrast, an approach that may be adequate to identify mature (rod-shaped) CMs, but is inefficient for the identification of immature (roundshaped) CMs with less-developed sarcomeres. By bypassing the use of a CM-specific marker, the authors most likely overestimated binucleation, particularly in resected hearts, which display, according to our results, higher rates of round-shaped mononucleated CMs. Cardiac fibroblasts are typically activated following injury, and their role in directing pathological disease remodeling is well recognized. Nonetheless, while adult fibroblasts induce hypertrophy, fetal fibroblasts promote CM proliferation and thus contribute to ventricular chamber enlargement in late development (leda et al., 2009). By combining the expression of three cell surface markers, we discriminated the main fibroblast subsets at different developmental stages and after cardiac injury. In response to injury, the fibroblast population, abundant at younger ages $\left(\mathrm{CD} 140 \mathrm{a}^{+} \mathrm{CD} 90^{+} \mathrm{SCA}-1^{-}\right)$, upregulated genes associated with the production of extracellular matrix, neovascularization, and secreted factors known to activate $\mathrm{CM}$ proliferation. In contrast, the fibroblast subset that is more frequent during adulthood $\left(\mathrm{CD} 140 \mathrm{a}^{+} \mathrm{CD} 90^{+} \mathrm{SCA}-1^{+}\right)$mainly upregulated transcripts associated with fibrotic mechanisms. Altogether, these findings suggest that neonatal fibroblasts may display an intermediate phenotype between fetal and adult fibroblasts, potentially able to support both fibrogenesis and regeneration.

Earlier studies on neonatal apex resection lacked functional longitudinal studies in which the same heart is analyzed over time. Herein we paralleled the histological analysis with 
detailed functional evaluation by echocardiography. In contrast to (Porrello et al., 2011), we observed the formation of fibrotic tissue in all analyzed hearts, which, in most cases, matured to a full-thickness scar as reported by others (Andersen et al., 2014a; Bryant et al., 2015). These contrasting observations are most likely a result of incomplete histological sampling in the former study, as shown previously (Andersen et al., 2014b). In our work, we increased sampling robustness by analyzing serial sections of the whole heart. Interestingly, while the heart weight and volume increased over time, as during normal heart growth, the scar volume diminished, indicating that the affected region was at least partially replaced by new myocardium. However, injured hearts remained shorter throughout the study, and thus the removed apex was not fully restored.

(Andersen et al., 2016) performed a long-term analysis of ventricular function, using F-18fluorodeoxyglucose positron emission tomography (FDG-PET). In this study, abnormal systolic function, short-axis enlargement and local wall thinning were demonstrated in resected hearts 180 days post-surgery, leading to the conclusion that animals developed dilated cardiomyopathy. Herein, although a tendency for reduced systolic function was evident 21 days post-surgery, no statistical differences were observed between groups over 180 days, indicating that injured hearts became functionally competent at the systolic level. Widening of resected hearts occurred through chamber expansion and LV wall thickening, which is not compatible with dilated cardiomyopathy, rather resembling an eccentric hypertrophy phenotype. The distinct results obtained from our work and (Andersen et al., 2016) may reflect differences in the methodology used. While FDG-PET is particularly valuable for assessing metabolic activity, the spatial resolution of the equipment $(\sim 1 \mathrm{~mm})$ falls short to precisely quantify LV wall thickness compared with the high-resolution echocardiography $(\sim 50 \mu \mathrm{m})$ (Gargiulo et al., 2012) used in our study. Moreover, a reduction in wall thickness was only significantly different in 1 of the 20 analyzed ventricular segments, which hardly explains a dilated cardiomyopathy phenotype. The authors also missed to demonstrate progressive loss of function, typically found in small animal models of this disease (Camacho et al., 2016).

Pertinent questions have been recently raised regarding the value of neonatal apex resection to identify factors important for adult heart regeneration/repair. Although we cannot put forward conclusions on the response elicited by other injury models, our results indicate that P1 hearts subjected to mechanical trauma are capable of undergoing neomyogenesis and neovascularization, despite local fibrosis (Figure 6). In our view, these findings endorse this injury model as particularly valuable for studies aiming to block fibrosis, in favor of conditions stimulating CM proliferation and functional cardiac regeneration. 


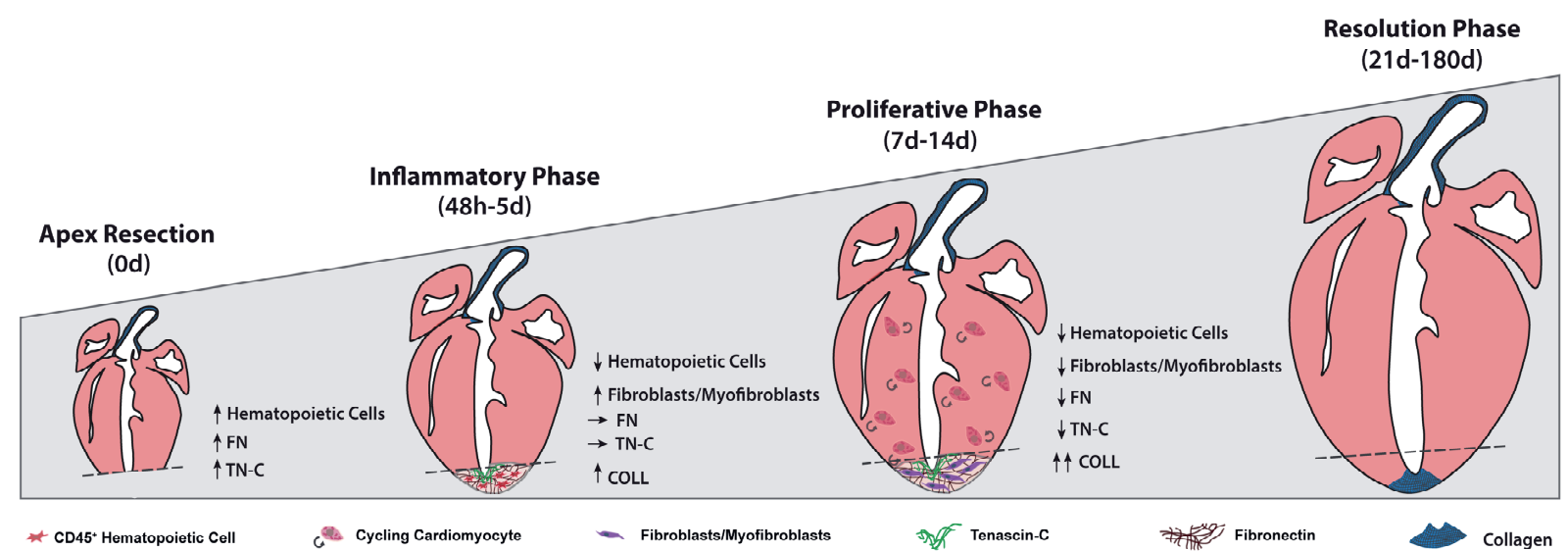

Figure 6. Proposed Model of the Biological Response Elicited by Neonatal Apex Resection

Apex resection promotes local infiltration of inflammatory cells in the first $48 \mathrm{~h}$, which leads to the deposition of a transient FN and TN-C-rich ECM. At 7 days post-injury, rates of CM proliferation are increased throughout the left ventricular myocardium and cardiac fibroblasts are activated at the injury site. These cellular dynamics result in a thickening of left ventricle walls, de novo vessel formation and deposition of a permanent fibrotic scar at the midpoint of the injured area. Long-term evaluation showed preserved systolic function, shortened long-axis and thicker left ventricle, without hypertrophy and edema.

\section{Experimental Procedures}

\section{Animals and Apex Resection Injury Model}

All animal work was approved by the IBMC-INEB (Instituto de Biologia Molecular e CelularInstituto de Engenharia Biomédica) Animal Ethics Committee, and by the Direcção Geral de Veterinária (permit 022793), and is in conformity with Directive 2010/63/EU. Humane endpoints were followed in accordance with the OECD Guidance Document on the Recognition, Assessment, and Use of Clinical Signs as Humane Endpoints for Experimental Animals Used in Safety Evaluation.

$\mathrm{P} 1 \mathrm{C} 57 \mathrm{BL} / 6$ mice were anesthetized by hypothermia for $\sim 3 \mathrm{~min}$. Animals were laid in lateral decubitus position, the skin cut, the thorax was opened in the fourth intercostal space and the ventricular apex was resected (removing a minimal amount of tissue but ensuring lumen exposure). Subsequently, the ribs and skin were sutured and animals were exposed to heat produced by an infrared lamp to revert anesthesia and returned the progenitor. Sham-operated animals underwent the same procedure with the exception of apex resection.

\section{$\underline{\text { Histological Characterization }}$}

At $0,7,14,21,60,120$, and 180 days after surgery, apex-resected and sham-operated hearts were harvested and processed for paraffin-embedding (see Supplemental Experimental Procedures for detailed information). Hearts were sectioned longitudinally in 
$3 \mu \mathrm{m}$ sections and sampled according to Figure S1A. Subsequent histological MT staining was used to assess apex re-growth, fibrosis deposition, and injury extension.

\section{$\underline{\text { Immunofluorescence }}$}

Immunofluorescence was performed in cryosections ( 0 day, 48 h, 5 days, 7 days, 14 days, and 21 days after surgery), in histological sections ( 60 days post-surgery), and in cytospins of isolated CMs; as detailed in the Supplemental Experimental Procedures. Assembly, merging, and contrast/brightness adjustments were performed in Fiji v2.0.0-rc-54/.51h. software.

\section{Flow Cytometry and Fluorescence-Activated Cell Sorting}

Single-cell suspensions were prepared following digestion of cardiac tissue with crude collagenase (C2139, Sigma-Aldrich). Cells were incubated with fluorescence-conjugated antibodies or with the respective isotype controls for $30 \mathrm{~min}$ on ice and protected from light. Nonviable cells were excluded by adding $0.5 \%$ propidium iodide (P4170, SigmaAldrich) to cell suspension prior to analysis in a FACSCanto II cytometer (BD Biosciences). Data analysis was performed in FlowJo VX software. The three most frequent fibroblast subsets were isolated by FACSAria (BD Biosciences), as described in Supplemental Experimental Procedures and in Figure S5. See also Figure S3 for detailed characterization of the inflammatory infiltrate.

\section{$\underline{\text { Real-Time } \mathrm{gPCR}}$}

Total RNA was isolated from FACS-sorted cells using the RNeasy Mini Kit (QIAGEN). cDNA was obtained resorting to PrimeScript RT reagent Kit (Takara) and pre-amplified using SsoAdvanced PreAmp Supermix (Bio-Rad). Real-time qPCR were performed by using iQ SYBR Green Supermix (Bio-Rad) and according to the iQ5 Real-Time PCR Detection System (Bio-Rad). Primer sequences and temperature cycles are described in Supplemental Experimental Procedures. mRNA expression was defined as primer efficiency to the power of the difference in threshold cycle values between the reference (Gapdh) and the genes of interest.

\section{EdU Pulse-Chase Assay}

Neonates were subcutaneously injected with $25 \mu \mathrm{g} / \mathrm{g}$ of animal of EdU (C10640, SigmaAldrich) immediately after surgery and on days 5 and 7 after surgery. Hearts were harvested at 14 days post-surgery and LV subdivided in apex and remote myocardium, each sampled in $2 \mathrm{~mm}$ fragments before cryosectioning $(40 \mu \mathrm{m})$. EdU incorporation was detected using the Click-iT reaction cocktail in accordance with manufacturer instructions (C10640, Sigma- 
Aldrich) followed by PCM-1 immunolabelling and DAPI counterstain. Images were acquired in a Leica TCS SP5 laser scanning confocal microscope. Tissue fragments (8-10) from both regions were quantified per animal with assistance of FIJI v2.0.0-rc-54/.51h and IMARIS 8.4.1. The total number of CMs was estimated by adapting the two-step $N_{V} \times V_{R E F}$ method (Alkass et al., 2015), where $\mathrm{N}_{\mathrm{V}}$ is an estimate of PCM-1 density and $\mathrm{V}_{\mathrm{REF}}$ is the volume of the myocardium at 14 days post-surgery. Myocardial volume was determined by multiplying the area of the myocardium in each histological longitudinal section by the distance separating adjacent sections in 14 days post-surgery hearts. This value was corrected for tissue shrinkage during fixation and processing for paraffin embedding.

\section{$\underline{\mathrm{CM} \text { Isolation }}$}

CMs were isolated at 7, 60, and 180 days post-surgery. Neonatal CMs were isolated as described previously (Mollova et al., 2013), with the following alterations: CMs were fixed in $4 \%$ paraformaldehyde at room temperature (RT) for $2 \mathrm{~h}$ and digested with $3 \mathrm{mg} / \mathrm{mL}$ collagenase type II (Worthington). Adult CMs were isolated by reperfusion in a Langendorff system ( $\sim 80 \mathrm{mmHg}$ and at $3 \mathrm{~mL} / \mathrm{min}$ for 25-30 min) with Liberase TM (05401127001, Roche; $13.3 \mu \mathrm{g} / \mathrm{mL}$ ) and Trypsin (27250018, Gibco; $13.8 \mu \mathrm{g} / \mathrm{mL}$ ), and fixed in $4 \%$ paraformaldehyde in PBS.

\section{Imaging Flow Cytometry}

Cells were permeabilized with $1 \times$ BD Perm/Wash buffer during $15 \mathrm{~min}$ at RT. Incubations with primary and secondary antibodies lasted 2 and $0.5 \mathrm{~h}$ on ice, respectively. Antibodies were diluted in $1 \times$ BD Perm/Wash. Immediately before acquisition in ImageStreamX cells were filtered and nuclei stained with $200 \mu \mathrm{M}$ DRAQ5 (BioStatus).

\section{Statistical Analysis}

IBM SPSS Statistics 24 was used for statistical analysis. Shapiro-Wilk test was used to evaluate normal distribution of data. Outliers were excluded from the statistical analysis. Homoscedasticity of the sample was tested by Levene's test, which defined the statistical test(s) applied. Normally distributed and homocedastic data were tested with independent sample Student's t test and one-way ANOVA for two or three or more groups, respectively. Tukey's post-hoc test for correction of multiple comparisons was performed whenever significant differences were found between three or more groups.

Non-normally distributed and/or heterocedastic data were tested with Mann-Whitney U test and Kruskal-Wallis one-way ANOVA for two or three or more groups, respectively. Again, multiple comparisons were performed when three or more groups were considered statistically different. The statistical significance level chosen for all statistical tests was $p<$ 0.05 . 


\section{Author Contributions}

V.S.-P. conceived, designed, and performed the experiments, analyzed and interpreted data, and wrote the manuscript. S.C.R., T.L.L., E.D.S., F.V.-N., A.C.S., R.J.C., N.P., T.P.R., and G.D. performed the experiments and analyzed and interpreted data. S.T. and A.L.-M. interpreted data and revised the manuscript. P.P.-Ó. conceived the study and interpreted data, revised the manuscript, and provided funding. D.S.N. conceived, designed, and performed the experiments, analyzed and interpreted data, revised the manuscript, and provided funding. All authors have read and approved the manuscript.

\section{Acknowledgments}

The authors acknowledge the support of i3S scientific platforms (animal facility, ALM, HEMS, BSU, b.IMAGE, CCGEN, TraCy). The authors are thankful to current and past members of Pinto-do-Ó laboratory for the critical discussion. This work was financed by Norte Portugal Regional Operational Programme (NORTE 2020), under the PORTUGAL 2020 Partnership Agreement, through the European Regional Development Fund (ERDF) (NORTE01-0145-FEDER-000012); by European Structural and Investment Funds (ESIF), under Lisbon Portugal Regional Operational Programme and National Funds through FCT (Fundação para a Ciência e Tecnologia [Foundation for Science and Technology] [POCl-01-0145-FEDER016385]); by INFARMED - Autoridade Nacional do Medicamento e Produtos de Saúde, I.P. (FIS-FIS-2015-01_CCV_20150630-157); and by FCT/Ministério da Ciência, Tecnologia e Inovação in the framework of the project "Institute for Research and Innovation in Health Sciences" (POCl-01-0145- FEDER-007274) and individual fellowships: SFRH/BD/111799/2015 to V.S.-P., PD/BD/127997/2016 to T.L.L., SFRH/BD/88780/2012 to A.C.S., and SFRH/BPD/80588/2011 to T.P.R.

\section{References}

Alkass, K., Panula, J., Westman, M., Wu, T.D., Guerquin-Kern, J.L., and Bergmann, O. (2015). No Evidence for Cardiomyocyte Number Expansion in Preadolescent Mice. Cell 163, 1026-1036.

Andersen, D.C., Ganesalingam, S., Jensen, C.H., and Sheikh, S.P. (2014a). Do neonatal mouse hearts regenerate following heart apex resection? Stem cell reports 2, 406-413.

Andersen, D.C., Jensen, C.H., Baun, C., Hvidsten, S., Zebrowski, D.C., Engel, F.B., and Sheikh, S.P. (2016). Persistent scarring and dilated cardiomyopathy suggest incomplete regeneration of the apex resected neonatal mouse myocardium--A 180 days follow up study. Journal of molecular and cellular cardiology 90, 47-52.

Andersen, D.C., Jensen, C.H., and Sheikh, S.P. (2014b). Response to Sadek et al. and Kotlikoff et al. Stem cell reports 3, 3-4.

Aurora, A.B., Porrello, E.R., Tan, W., Mahmoud, A.I., Hill, J.A., Bassel-Duby, R., Sadek, H.A., and Olson, E.N. (2014). Macrophages are required for neonatal heart regeneration. The Journal of clinical investigation 124, 1382-1392.

Bassat, E., Mutlak, Y.E., Genzelinakh, A., Shadrin, I.Y., Baruch Umansky, K., Yifa, O., Kain, D., Rajchman, D., Leach, J., Riabov Bassat, D., et al. (2017). The extracellular matrix protein agrin promotes heart regeneration in mice. Nature 547, 179-184.

Bersell, K., Arab, S., Haring, B., and Kuhn, B. (2009). Neuregulin1/ErbB4 signaling induces cardiomyocyte proliferation and repair of heart injury. Cell 138, 257-270. 
Bryant, D.M., O'Meara, C.C., Ho, N.N., Gannon, J., Cai, L., and Lee, R.T. (2015). A systematic analysis of neonatal mouse heart regeneration after apical resection. Journal of molecular and cellular cardiology 79, 315-318.

Camacho, P., Fan, H., Liu, Z., and He, J.Q. (2016). Small mammalian animal models of heart disease. American journal of cardiovascular disease 6, 70-80.

Collesi, C., Zentilin, L., Sinagra, G., and Giacca, M. (2008). Notch1 signaling stimulates proliferation of immature cardiomyocytes. The Journal of cell biology 183, 117-128.

D'Uva, G., Aharonov, A., Lauriola, M., Kain, D., Yahalom-Ronen, Y., Carvalho, S., Weisinger, K., Bassat, E., Rajchman, D., Yifa, O., et al. (2015). ERBB2 triggers mammalian heart regeneration by promoting cardiomyocyte dedifferentiation and proliferation. Nature cell biology 17, 627-638.

Darehzereshki, A., Rubin, N., Gamba, L., Kim, J., Fraser, J., Huang, Y., Billings, J., Mohammadzadeh, R., Wood, J., Warburton, D., et al. (2015). Differential regenerative capacity of neonatal mouse hearts after cryoinjury. Developmental biology 399, 91-99.

Flink, I. (2002). Cell cycle reentry of ventricular and atrial cardiomyocytes and cells within the epicardium following amputation of the ventricular apex in the axolotl, Amblystoma mexicanum : confocal microscopic immunofluorescent image analysis of bromodeoxyuridine-labeled nuclei. Anatomy and embryology 205, 235-244. Furtado, M.B., Costa, M.W., Pranoto, E.A., Salimova, E., Pinto, A.R., Lam, N.T., Park, A., Snider, P., Chandran, A., Harvey, R.P., et al. (2014). Cardiogenic genes expressed in cardiac fibroblasts contribute to heart development and repair. Circulation research 114, 1422-1434.

Gargiulo, S., Greco, A., Gramanzini, M., Petretta, M.P., Ferro, A., Larobina, M., Panico, M., Brunetti, A., and Cuocolo, A. (2012). PET/CT imaging in mouse models of myocardial ischemia. Journal of biomedicine \& biotechnology 2012, 541872.

Haubner, B.J., Adamowicz-Brice, M., Khadayate, S., Tiefenthaler, V., Metzler, B., Aitman, T., and Penninger, J.M. (2012). Complete cardiac regeneration in a mouse model of myocardial infarction. Aging 4, 966-977.

Heallen, T., Morikawa, Y., Leach, J., Tao, G., Willerson, J.T., Johnson, R.L., and Martin, J.F. (2013). Hippo signaling impedes adult heart regeneration. Development (Cambridge, England) 140, 4683-4690.

Hudon-David, F., Bouzeghrane, F., Couture, P., and Thibault, G. (2007). Thy-1 expression by cardiac fibroblasts: lack of association with myofibroblast contractile markers. Journal of molecular and cellular cardiology 42, 9911000 .

leda, M., Tsuchihashi, T., Ivey, K.N., Ross, R.S., Hong, T.T., Shaw, R.M., and Srivastava, D. (2009). Cardiac fibroblasts regulate myocardial proliferation through beta1 integrin signaling. Developmental cell 16, 233-244.

Jesty, S.A., Steffey, M.A., Lee, F.K., Breitbach, M., Hesse, M., Reining, S., Lee, J.C., Doran, R.M., Nikitin, A.Y., Fleischmann, B.K., et al. (2012). c-kit+ precursors support postinfarction myogenesis in the neonatal, but not adult, heart. Proceedings of the National Academy of Sciences of the United States of America 109, 13380-13385.

Kotlikoff, M.I., Hesse, M., and Fleischmann, B.K. (2014). Comment on "Do neonatal mouse hearts regenerate following heart apex resection"? Stem cell reports 3, 2.

Lavine, K.J., Epelman, S., Uchida, K., Weber, K.J., Nichols, C.G., Schilling, J.D., Ornitz, D.M., Randolph, G.J., and Mann, D.L. (2014). Distinct macrophage lineages contribute to disparate patterns of cardiac recovery and remodeling in the neonatal and adult heart. Proceedings of the National Academy of Sciences of the United States of America 111, 16029-16034.

Mahmoud, A.I., Kocabas, F., Muralidhar, S.A., Kimura, W., Koura, A.S., Thet, S., Porrello, E.R., and Sadek, H.A. (2013). Meis1 regulates postnatal cardiomyocyte cell cycle arrest. Nature 497, 249-253.

Mahmoud, A.I., Porrello, E.R., Kimura, W., Olson, E.N., and Sadek, H.A. (2014). Surgical models for cardiac regeneration in neonatal mice. Nat Protoc 9, 305-311.

McQualter, J.L., Brouard, N., Williams, B., Baird, B.N., Sims-Lucas, S., Yuen, K., Nilsson, S.K., Simmons, P.J., and Bertoncello, I. (2009). Endogenous fibroblastic progenitor cells in the adult mouse lung are highly enriched in the sca-1 positive cell fraction. Stem cells (Dayton, Ohio) 27, 623-633.

Mollova, M., Bersell, K., Walsh, S., Savla, J., Das, L.T., Park, S.Y., Silberstein, L.E., Dos Remedios, C.G., Graham, D., Colan, S., et al. (2013). Cardiomyocyte proliferation contributes to heart growth in young humans. Proceedings of the National Academy of Sciences of the United States of America 110,1446-1451.

Mozaffarian, D., Benjamin, E.J., Go, A.S., Arnett, D.K., Blaha, M.J., Cushman, M., Das, S.R., de Ferranti, S., Despres, J.P., Fullerton, H.J., et al. (2016). Heart Disease and Stroke Statistics-2016 Update: A Report From the American Heart Association. Circulation 133, e38-360. 
Paradis, A.N., Gay, M.S., and Zhang, L. (2014). Binucleation of cardiomyocytes: the transition from a proliferative to a terminally differentiated state. Drug Discov Today 19, 602-609.

Porrello, E.R., Mahmoud, A.I., Simpson, E., Hill, J.A., Richardson, J.A., Olson, E.N., and Sadek, H.A. (2011). Transient regenerative potential of the neonatal mouse heart. Science (New York, NY) 331, 1078-1080.

Porrello, E.R., Mahmoud, A.I., Simpson, E., Johnson, B.A., Grinsfelder, D., Canseco, D., Mammen, P.P., Rothermel, B.A., Olson, E.N., and Sadek, H.A. (2013). Regulation of neonatal and adult mammalian heart regeneration by the miR-15 family. Proceedings of the National Academy of Sciences of the United States of America 110, 187-192.

Poss, K.D., Wilson, L.G., and Keating, M.T. (2002). Heart regeneration in zebrafish. Science (New York, NY) 298, 2188-2190.

Robledo, M. (1956). Myocardial regeneration in young rats. The American journal of pathology 32, 1215-1239.

Sadek, H.A., Martin, J.F., Takeuchi, J.K., Leor, J., Nie, Y., Giacca, M., and Lee, R.T. (2014). Multi-investigator letter on reproducibility of neonatal heart regeneration following apical resection. Stem cell reports $3,1$.

Smith, C.L., Baek, S.T., Sung, C.Y., and Tallquist, M.D. (2011). Epicardial-derived cell epithelial-to-mesenchymal transition and fate specification require PDGF receptor signaling. Circulation research 108, e15-26.

Soonpaa, M.H., Kim, K.K., Pajak, L., Franklin, M., and Field, L.J. (1996). Cardiomyocyte DNA synthesis and binucleation during murine development. The American journal of physiology 271, H2183-2189.

Souders, C.A., Bowers, S.L., and Baudino, T.A. (2009). Cardiac fibroblast: the renaissance cell. Circulation research $105,1164-1176$.

Tidball, J.G. (2011). Mechanisms of muscle injury, repair, and regeneration. Comprehensive Physiology 1, 20292062.

Uygur, A., and Lee, R.T. (2016). Mechanisms of Cardiac Regeneration. Developmental cell 36, 362-374.

Zebrowski, D.C., Becker, R., and Engel, F.B. (2016). Towards regenerating the mammalian heart: challenges in evaluating experimentally induced adult mammalian cardiomyocyte proliferation. American journal of physiology Heart and circulatory physiology 310, H1045-1054.

Zebrowski, D.C., Jensen, C.H., Becker, R., Ferrazzi, F., Baun, C., Hvidsten, S., Sheikh, S.P., Polizzotti, B.D., Andersen, D.C., and Engel, F.B. (2017). Cardiac injury of the newborn mammalian heart accelerates cardiomyocyte terminal differentiation. Sci Rep 7, 8362. 


\section{Supplemental Material}

\section{Supplemental Figure 1}

A

$$
\begin{gathered}
\hookrightarrow \overleftrightarrow{40}(0 d) \\
50 \mu \mathrm{m}(7 d) \\
70 \mu \mathrm{m}(14 d) \\
90 \mu \mathrm{m}(21 d) \\
130 \mu \mathrm{m}(60 \mathrm{~d}, 180 \mathrm{~d})
\end{gathered}
$$

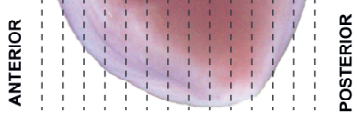

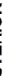

B

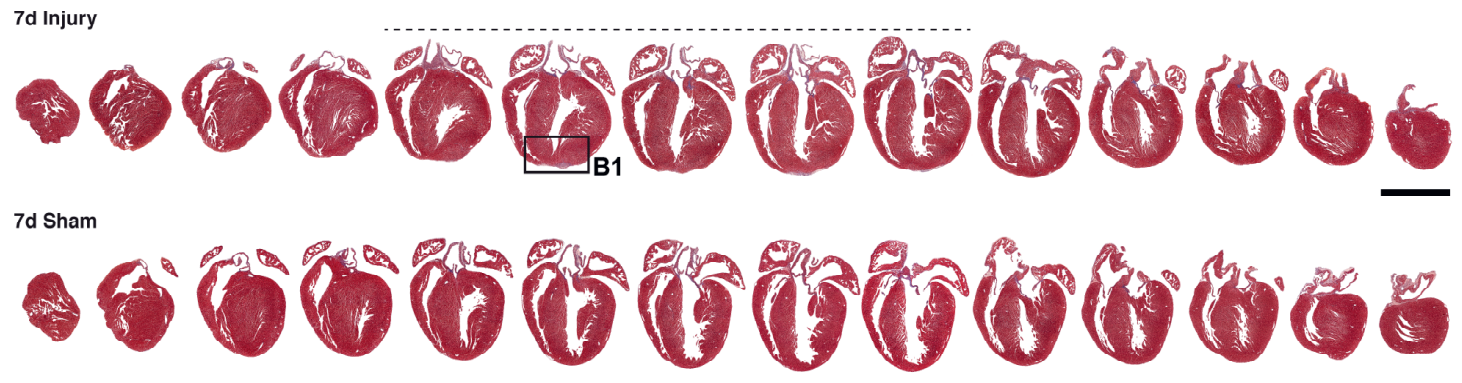

14d Injury

-

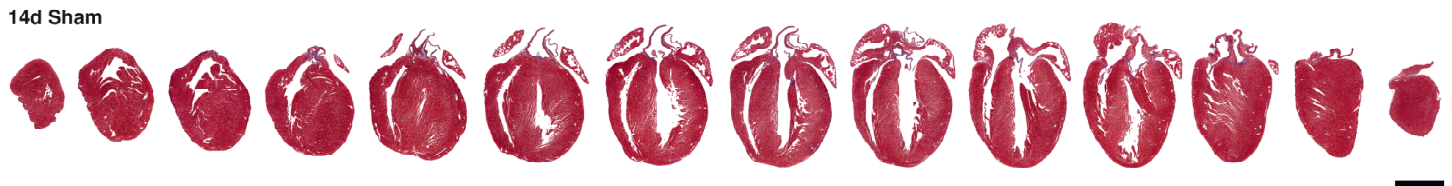
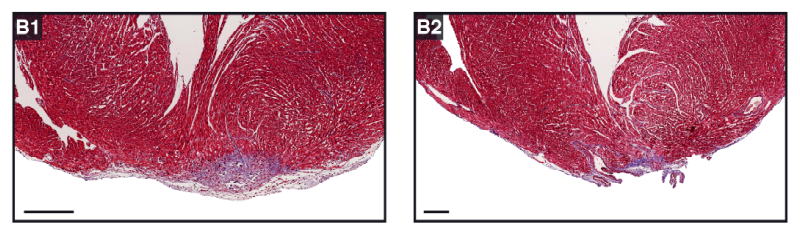

C
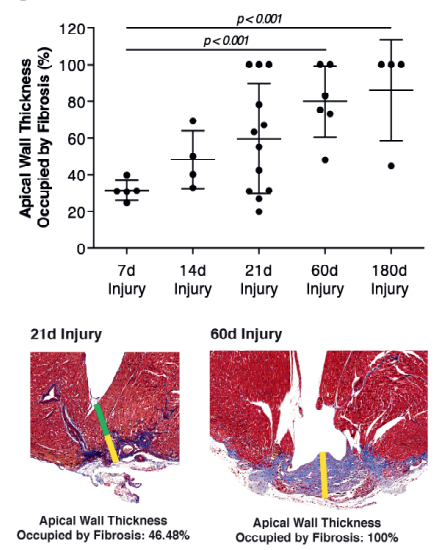

D

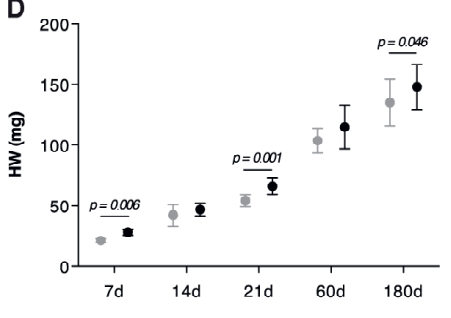

- Injury
E

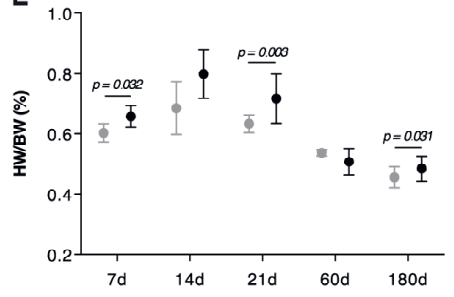


Neonatal apex resection triggers cardiomyocyte proliferation, neovascularization and functional recovery despite local fibrosis

$N$
0
$\frac{1}{3}$
$\frac{00}{20}$
$\frac{\pi}{0}$
$\stackrel{2}{0}$
$\frac{0}{0}$
$\frac{c}{0}$
$\frac{0}{2}$
$\frac{2}{3}$
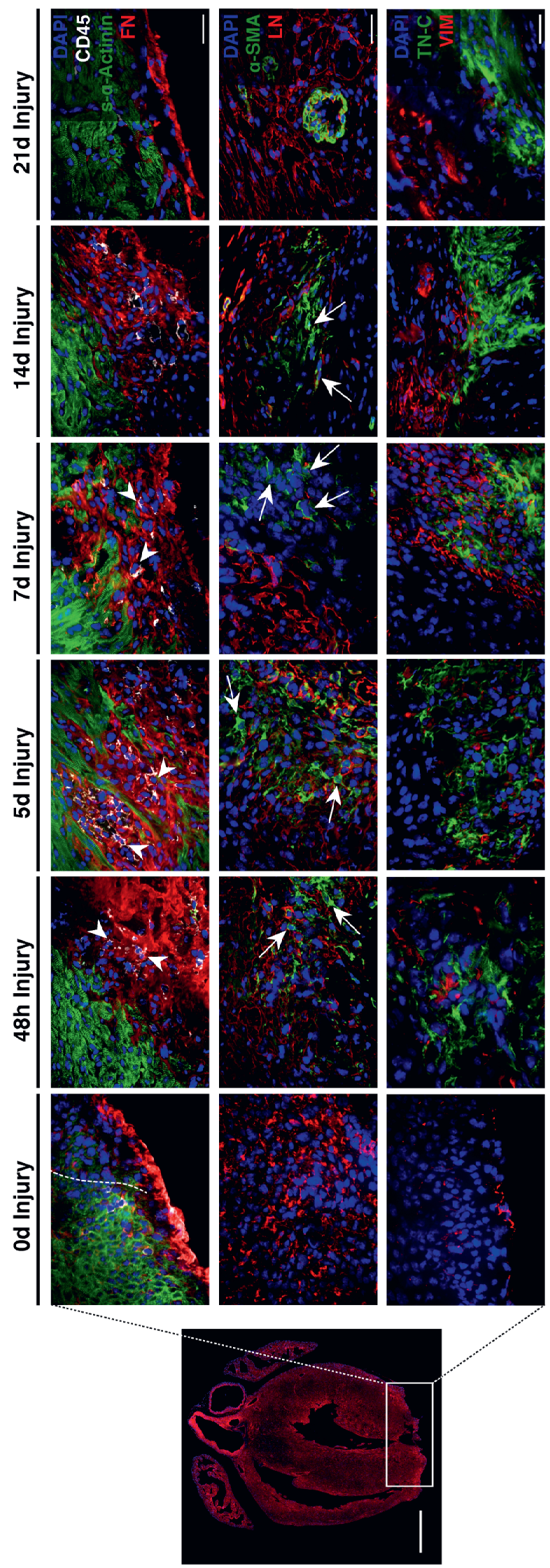


\section{Supplemental Figure 3}

A
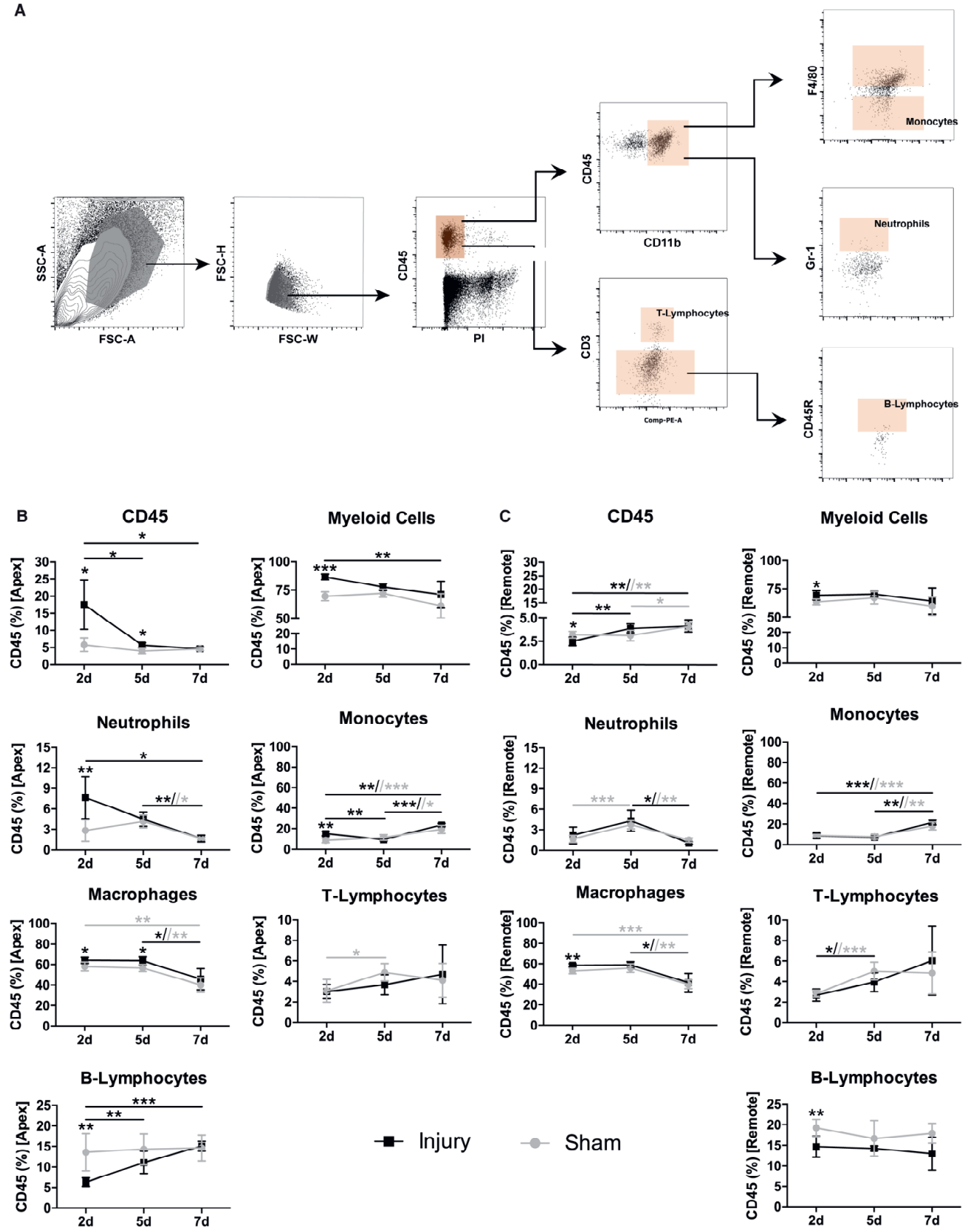

D Ly6C ${ }^{\text {hi Monocytes }}$
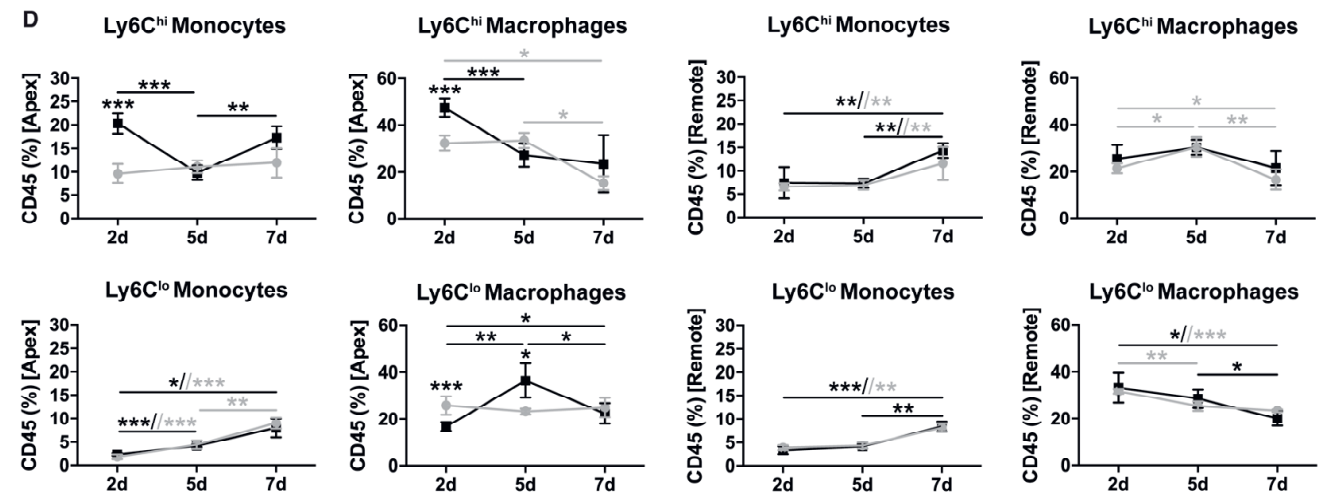
Supplemental Figure 4
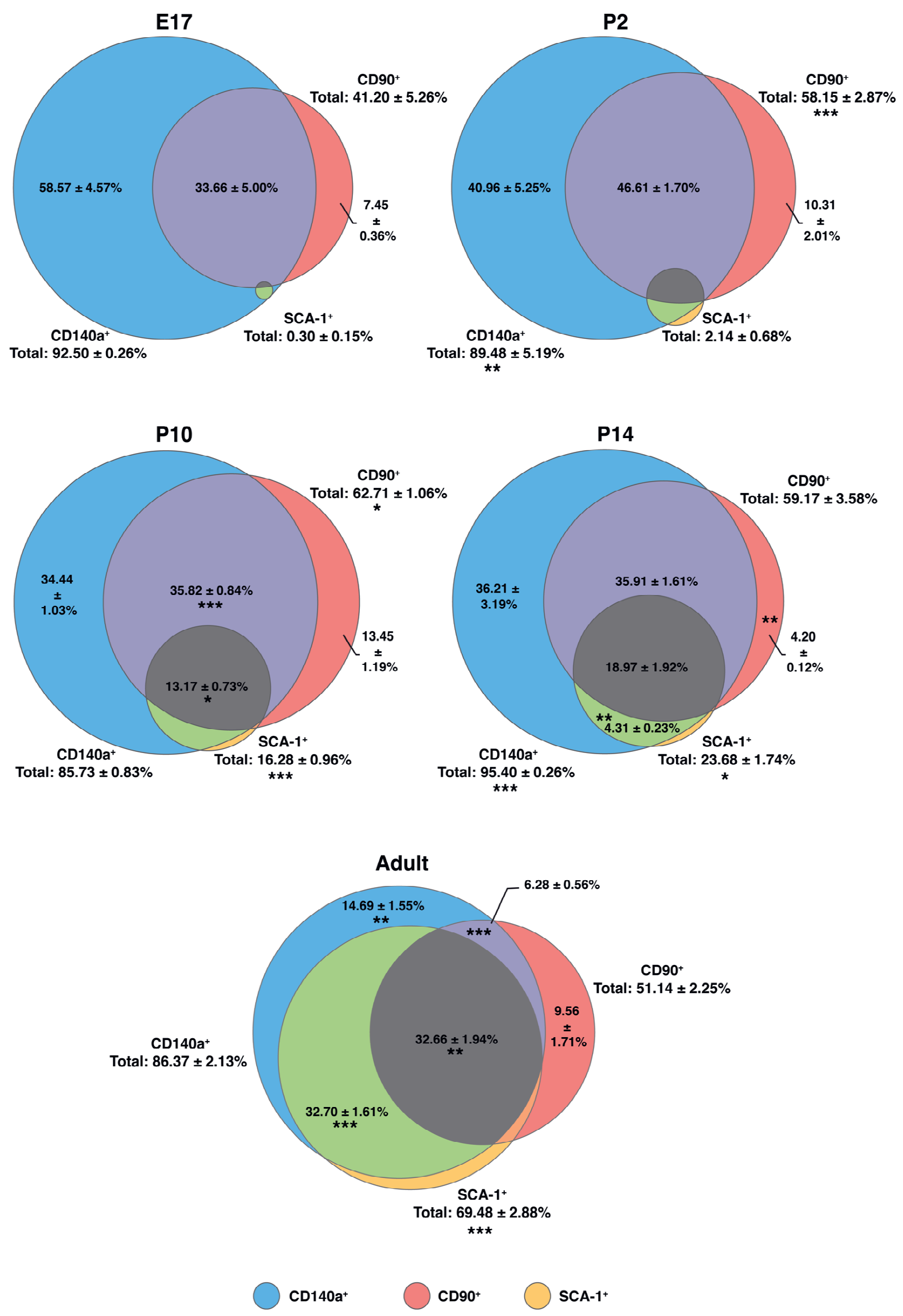
Supplemental Figure 5
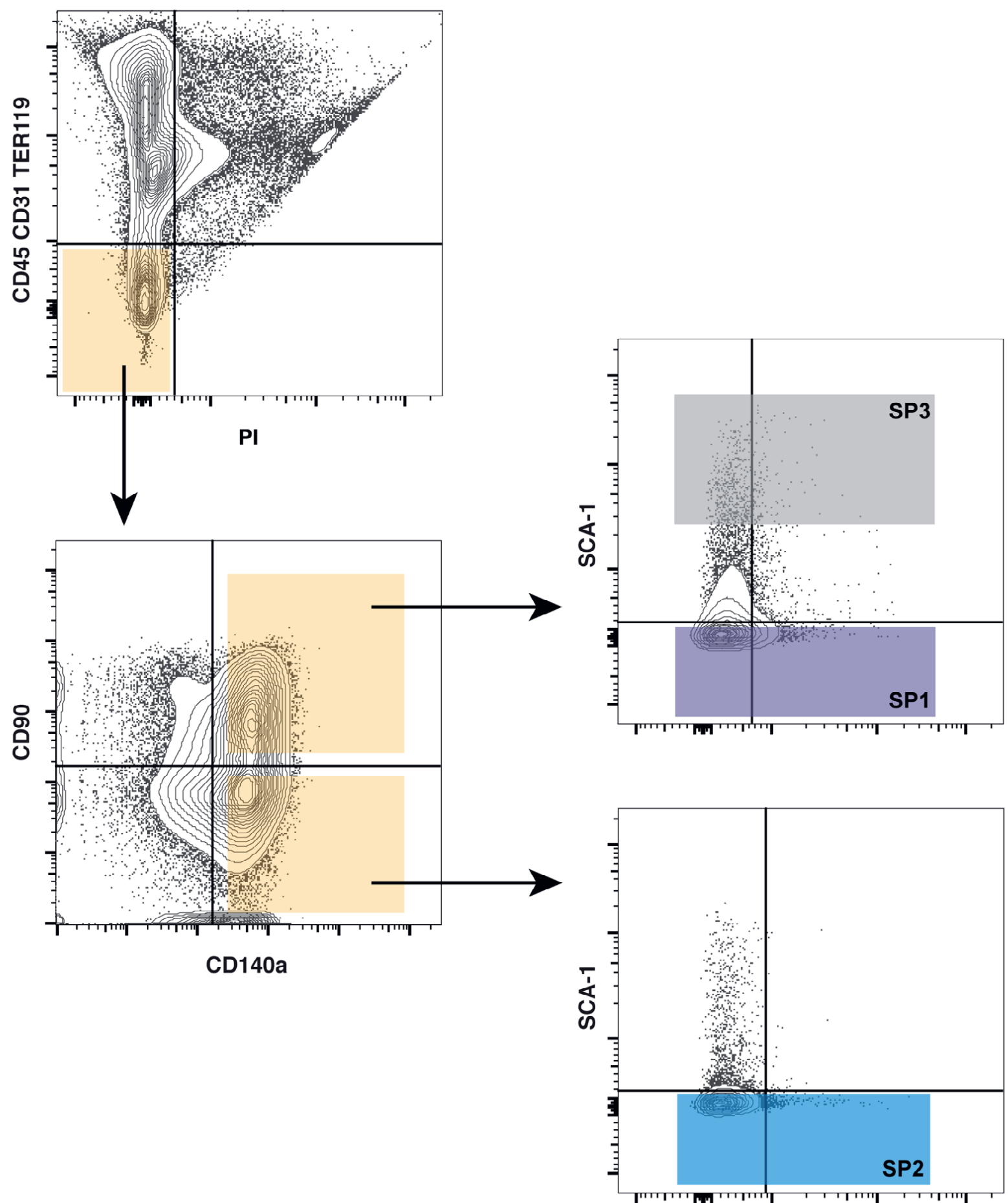
Neonatal apex resection triggers cardiomyocyte proliferation, neovascularization and functional recovery despite local fibrosis

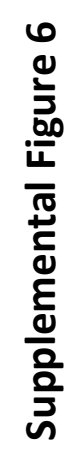

$\boldsymbol{m}$
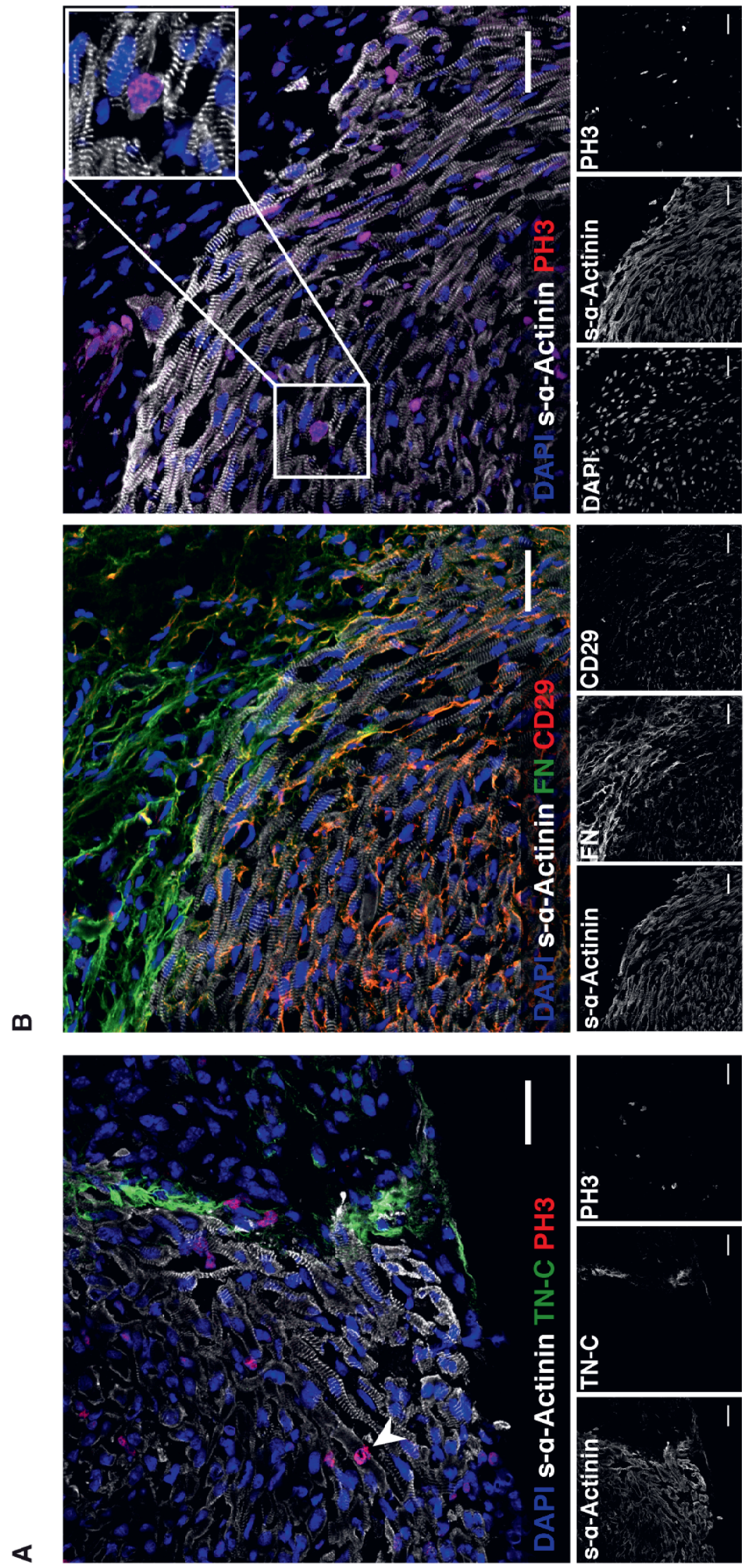
Supplemental Figure 1. Schematic Representation of Heart Sampling (Related to Experimental Procedures) \& Cardiac Remodeling After Neonatal Apex Resection (Related to Figure 1)

(A) Representative sectioning was adjusted to the heart size (i.e. $40 \mu \mathrm{m}, 50 \mu \mathrm{m}, 70 \mu \mathrm{m}, 90 \mu \mathrm{m}$ and $130 \mu \mathrm{m}$, between each section for $0 d, 7 d, 14 d, 21 d$ and $60 / 180 d$ post resection, respectively), yielding approximately the same number of sections. (B) Representative MT stained sections of apex resected and surgical controls 7 and $14 d$ following surgery. Sections exhibiting myocardial disruption and/or cardiac fibrosis are highlighted by a dashed line (scale bars, $2 \mathrm{~mm}$ ). High magnification images of the injury site (B1: 7d, B2: 14d) show collagen (blue staining) (scale bars, $250 \mu \mathrm{m})$. (C) Percentage of left ventricle wall occupied by fibrosis in apex region was determined at $7 \mathrm{~d}$ $(n=5), 14 d(n=4), 21 d(n=12), 60 d(n=6)$ and $180 d(n=4)$. (D and E) Heart weight (from left to right, $n=4,7,3,4$, $8,10,5,7,19,17$ ) and heart to body weight ratio (from left to right, $n=4,7,3,4,8,12,3,7,19,17$ ). All values are presented as means \pm SD.

Supplemental Figure 2. Time-specific Deposition of Extracellular Matrix Correlates with Cellular Dynamics (Related to Figure 2)

Arrowheads: $\mathrm{CD}^{+} 5^{+}$hematopoietic cells; arrows: $\alpha \mathrm{SMA}^{+}$myofibroblasts; Low magnification image (scale bar, $500 \mu \mathrm{m})$, High magnification images (scale bars, $30 \mu \mathrm{m})(\mathrm{n}=4$ for each time point).

Supplemental Figure 3. Characterization of the Inflammatory Infiltrate After Injury

(A) Representative plots showing the discrimination of hematopoietic (CD45 ) subsets after surgery. (B) Relative percentage of immune cell populations in the apex of apex-resected and sham-operated animals at $2 \mathrm{~d}, 5 \mathrm{~d}$ and $7 \mathrm{~d}$ post-surgery. (C) Relative percentage of immune cell populations in the remote myocardium of apex-resected and sham-operated animals at $2 d, 5 d$ and $7 d$ post-surgery. (D) Relative percentage of monocytes and macrophages according to Ly6C expression in the apex and remote myocardium at $2 d, 5 d$ and $7 d$ post-surgery. $(n=7,5,4,4,3$, 3; $2 d$ sham, $2 d$ injury, $5 d$ sham, $5 d$ injury, $7 d$ sham, $7 d$ injury). ( $\left.{ }^{*} p<0.05 ;{ }^{* *} p<0.01 ; * * p<0.001\right)$. All values are presented as means \pm SD.

Supplemental Figure 4. Phenotypic Characterization of Cardiac Fibroblasts Throughout Ontogeny (Related to Figure 3)

Discrimination of fibroblasts based on the combined expression of CD140a, CD90 and SCA-1 at E17 ( $\mathrm{n}=2$ [2 pools of 3 hearts]), P2 $(n=3)$, P10 $(n=3) ;$ P14 $(n=5)$ and adulthood $(n=4)$. Statistical differences $\left({ }^{*} p<0.05 ; * * p<0.01\right.$; $\left.{ }^{* * *} p<0.001\right)$ of each population between consecutive time points are shown. All values are presented as means \pm SEM.

Supplemental Figure 5. Sorting Strategy to Isolate Activated Cardiac Fibroblast Populations (Related to Figure 3 ) SP1, SP2 and SP3 subsets were sorted following removal of non-viable cells $\left(\mathrm{PI}^{+}\right)$, hematopoietic cells $\left(\mathrm{CD} 45^{+}\right)$, endothelial cells $\left(C D 31^{+}\right)$and erythrocytes $($TER119+) $(n=5,4 ; 7 d$ sham, $7 d$ injury).

Supplemental Figure 6. CM Proliferation and ECM Production at 7d After Apex Resection (Related to Figures 3 and 4) Representative images of consecutive sections stained for s-a-Actinin/FN/CD29 and s-a-Actinin/PH3 ( $n=10)$ at 7d post-surgery. High magnification image highlights a proliferating $\mathrm{CM}$ located in a region marked by the deposition of FN and by the expression of CD29 in CMs (Scale bars, 30 $\mu \mathrm{m}$ ). 


\section{Supplemental Table 1}

Lead-II Electrocardiographic parameters at 180d post-surgery (Related to Figure 2)

\begin{tabular}{|c|c|c|c|}
\hline Variables & $\begin{array}{c}\text { 180d Sham (Mean } \pm \\
\text { SEM) } \mathbf{n = 1 0}\end{array}$ & $\begin{array}{c}\text { 180d Injury (Mean } \pm \\
\text { SEM) } \mathbf{n = 6}\end{array}$ & P-Value \\
\hline RR Interval (s) & $0,1275 \pm 0,0031$ & $0,1265 \pm 0,0012$ & $\begin{array}{c}0,875 \text { (Mann- } \\
\text { Whitney U Test) }\end{array}$ \\
\hline Heart Rate (bpm) & $474,01 \pm 11,34$ & $475,02 \pm 4,67$ & $\begin{array}{c}1,000 \text { (Mann- } \\
\text { Whitney U Test) }\end{array}$ \\
\hline PR Interval (s) & $0,0407 \pm 0,0064$ & $0,0442 \pm 0,0020$ & 0,065 (T-Test) \\
\hline P Duration (s) & $0,0143 \pm 0,0008$ & $0,0153 \pm 0,0010$ & 0,447 (T-Test) \\
\hline QRS Interval (s) & $0,0095 \pm 0,0003$ & $0,0093 \pm 0,0034$ & 0,928 (T-Test) \\
\hline QT Interval (s) & $0,0131 \pm 0,0004$ & $0,0142 \pm 0,0006$ & 0,141 (T-Test) \\
\hline QTc (s) ${ }^{*}$ & $0,0367 \pm 0,0014$ & $0,0399 \pm 0,0018$ & 0,189 (T-Test) \\
\hline JT Interval (s) & $0,0033 \pm 0,0003$ & $0,0040 \pm 0,0006$ & 0,285 (T-Test) \\
\hline Tpeak Tend Interval (s) & $0,0021 \pm 0,0003$ & $0,0026 \pm 0,0005$ & 0,391 (T-Test) \\
\hline P Amplitude (mV) & $0,0697 \pm 0,2179$ & $0,0634 \pm 0,0263$ & 0,607 (Mann- \\
& & & Whitney U Test) \\
\hline Q Amplitude (mV) & $0,0078 \pm 0,0102$ & $-0,0552 \pm 0,0238$ & 0,056 (Mann- \\
& & & Whitney U Test) \\
\hline R Amplitude (mV) & $1,1664 \pm 0,1572$ & $1,1284 \pm 0,1848$ & 0,881 (T-Test) \\
\hline S Amplitude (mV) & $-0,8133 \pm 0,0700$ & $-0,7484 \pm 0,0863$ & 0,374 (Mann- \\
& & & Whitney U Test) \\
\hline ST Height (mV) & $-0,0363 \pm 0,0291$ & $-0,0390 \pm 0,0224$ & 0,950 (T-Test) \\
\hline T Amplitude (mV) & $0,1860 \pm 0,0327$ & $0,1100 \pm 0,0399$ & 0,179 (T-Test) \\
\hline
\end{tabular}

* QT interval was adjusted to heart rate (QTc) using Bazett's formula.

$s$ - seconds

bpm - beats per minute

$\mathrm{mV}-$ millivolt

\section{Supplemental Experimental Procedures}

\section{Neonatal Apex Resection and Sham-Surgery}

The neonatal injury model consists of the apex surgical resection of post-natal day $(P) 1$ C57BL/6 mice. Animals were anesthetized by hypothermia during three minutes ( $\mathrm{min}$ ) to cease cardiorespiratory movements. Animals were laid in lateral decubitus position, exposing the left side of the flank. After immobilization with sealing tape, the skin was cut and muscle fibers disrupted until the ribs were observed. The thoracic cavity was opened in the $4^{\text {th }}$ intercostal space and the left ventricle apex cut with fine scissors until chamber exposure. Thorax and skin were closed by 7-0 absorbable suture (Coated Vicryl Ethicon). 
Animals recovered from anesthesia under an infrared lamp and were subjected to stimulation by tightening the paw, when necessary. The surgical procedure was completed when animals regained regular breathing. Neonates were returned to the progenitor cage immediately after all the litter was intervened. During all the procedure, except throughout surgery, neonates were warmed by warming pads and infrared light. Sham mice underwent the exact same procedure with the exception of apex resection (any kind of heart manipulation whatsoever).

\section{$\underline{\text { Histological Assessment }}$}

Hearts were harvested at $0,7,14,21,60$ and 180 days post-surgery (d injury/sham). Hearts were submersed in phosphate buffer saline (PBS) and fixed in $10 \%$ formalin neutral buffer (VWR BDH \& Prolabo) during 16-24 hours at room temperature (RT). For paraffin embedding, hearts were processed during a total time of 12 hours in an automated system through successive PBS washes, increasing series of alcohols (Aga), Clear Rite $3^{\circledR}$ (RichardAllan Scientific) and Shandon Histoplast (Thermo Scientific) at $56^{\circ} \mathrm{C}$. Hearts were included in paraffin and sectioned (microtome RM2255, Leica) longitudinally (3 $\mu \mathrm{m}$ sections) according to Figure S1. Sections were dewaxed and rehydrated prior to modified Masson's Trichrome (MT) staining. The MT was performed as in the Trichrome (Masson) Stain kit (HT15-1KT, Sigma-Aldrich), with the following modifications: nuclei were prestained with Celestine Blue and with Gill's Hematoxylin, followed by an incubation in aqueous Bouin solution for 1 hour to promote uniform staining. Sections were diafanized in xylene and mounted in DPX Mountant for histology (06522, Sigma-Aldrich $\left.{ }^{\circledR}\right)$. Images were acquired in the Hamamatsu Nanozoomer 2.0-HT digital slide scanner and subsequent editing was performed in NDP view 2 software (Hamammatsu).

\section{Morphometric Analysis}

Resorting to MT stained sections and with assistance of FIJI v2.0.0-rc-54/.51h software, morphometric analysis was performed as follows:

1. Ventricular Surface Area: measurement of the whole ventricle cross-sectional area (i.e excluding great vessels, atria and tissue adherences in the apex region);

2. Heart Length/BW: the distance between the base of the heart (region corresponding to the insertion of the great vessels in the myocardium) and the apex was normalized by the body-weight.

3. Injury (\%): the percentage of sections collected from a single heart demonstrating myocardial disruption and/or fibrosis.

4. Ventricle/Scar Volume: ventricle and scarring area multiplied by the distance separating adjacent sections.

5. Apical Wall Thickness Occupied by Fibrosis (\%): percentage of the left ventricle wall thickness at the apical region occupied by fibrosis. 
6. Cardiomyocyte cross-sectional area: the area of transversally cut cardiomyocytes was determined in at least 30 cardiomyocytes of the apical and remote myocardium per section (at least 8 sections were analyzed per heart).

\section{Functional Characterization}

Animals at $21,60,120$ and $180 \mathrm{~d}$ post-surgery were subjected to echocardiography using the Vevo2100 system and a $40 \mathrm{MHz}$ probe (Visualsonics). Anesthesia was induced in a chamber filled with $5 \%$ isoflurane (IsoVet, Braun) and verified by loss of body posture and the paw withdrawal reflex. The animal was transferred to a heated support and the state of anesthesia was maintained through a face mask ( $1.5 \%$ isoflurane). Animal fur was shaved and mice paws were placed over sensors and in contact with an electric-conductive gel to monitor heart and respiratory rates. Body temperature was assessed using a rectal probe. Two-dimensional images of the heart were acquired focusing both short-axis (SAX) and parasternal long-axis (PSLAX) views. Motion-mode (M-Mode) was conducted in the SAX view to determine the thickness of LV walls as well as chamber diameter during systole and diastole. Ejection fraction (EF) was determined by Simpson's method: a longitudinal segment and three transverse segments were traced in the distal, medial and proximal region of the heart in PSLAX view, which assisted on the determination of heart volume during diastole and systole.

Vol d,s $=\frac{\left(\pi\left(\frac{\text { distal }, s}{2}\right)^{2}+\left(\pi\left(\frac{\text { medial }, s}{2}\right)^{2}+\left(\pi\left(\frac{\text { proximal }, s}{2}\right)^{2}\right) \times \text { Hearth Length }, s\right.\right.}{3}$

Stroke volume (SV) was determined by the difference between diastolic and systolic volume. Conversely, ejection fraction was determined as follows:

$$
E F=\frac{S V}{\operatorname{Vold}} \times 100
$$

Cardiac output (CO) was calculated by multiplying SV by heart rate (HR).

Eccentricity index was determined by dividing left ventricle diameter (at the level of papillary muscles) by the longitudinal heart length (distance from the apex to great vessel insertion in the ventricle), both obtained in PSLAX during diastole.

Diastolic function was evaluated by the ratio between early $(E)$ to late (A) filling velocities of the left ventricle.

Myocardial performance index (also known as Tei index) incorporates both systolic (IVCT isovolumetric contraction time) and diastolic (IVRT - isovolumetric relaxation time) time intervals:

$$
M P I=\frac{I V C T+I V R T}{\text { Aortic Ejection Time }}
$$


By comparing these factors on sham operated and injured hearts the degree of functional restoration was ascertained.

Electrophysiological parameters were also assessed at $180 \mathrm{~d}$ post-surgery. Anesthesia was induced and confirmed as described for echocardiographic evaluation. Animals were placed in supine position and intradermal electrodes were placed in a lead II-like configuration. ECG signals were obtained with a data acquisition hardware (PowerLab 8/35, ADInstruments) coupled to a signal amplifier (Animal Bio Amp, ADInstruments), with support of LabChart 8 software (ADInstruments).

Using an ECG analysis module RR Interval, HR, PR Interval, P Duration, QRS Interval, QT Interval, JT Interval, T-peak to T-end interval, the amplitudes of $P, Q, R, S$ and $T$ waves and the ST segment height were determined.

Furthermore, QT corrected for the HR (QTC) was calculated through the Bazett's formula:

$$
Q T c=\frac{Q T}{\sqrt{\text { RR interval }}}
$$

\section{$\underline{\text { Immunofluorescence }}$}

Both cryo and paraffin sections as well as cytospins were used to evaluate protein expression. Three $\mu \mathrm{m}$ paraffin sections were dewaxed and rehydrated by three changes in xylene, followed by sequential alcohol gradients and rinsing in deionized water. If antigen retrieval was necessary, heat-induced epitope retrieval (HIER) was applied. For HIER, sections were incubated for $35 \mathrm{~min}$ at $98^{\circ} \mathrm{C}$ in a water bath, in $10 \mathrm{mM}$ Tris $1 \mathrm{mM}$ EDTA (TrisEDTA), pH 9.0 and allowed to cool for $20 \mathrm{~min}$ at room temperature.

Tissue processing for cryosectioning involves 3 consecutive submersions (each during 24 hours at $4^{\circ} \mathrm{C}$ ) in different solutions: $0,2 \%$ Paraformaldehyde (PFA) in 0,12M phosphate buffer (PB); $4 \%$ sucrose in $0,12 \mathrm{M} \mathrm{PB}$ and $15 \%$ sucrose in $0,12 \mathrm{M} \mathrm{PB}$. A fourth incubation is performed in $15 \%$ sucrose and $7,5 \%$ gelatin in $0,12 \mathrm{M} \mathrm{PB}$ during 1 hour at $37^{\circ} \mathrm{C}$. Hearts were transferred to molds containing the last solution (warmed) and placed on top of dry-icechilled 2-methylbutane (GPR Rectapur, VWR). Frozen hearts were stored at $-80^{\circ} \mathrm{C}$ before being cut from one end to the other into $5 \mu \mathrm{m}$ sections using a cryomicrotome (Microtom HM 550, Thermo Scientific).

When targeting epitopes that required cell membrane permeabilization, sections were treated with $0.2 \%$ Triton X-100 (for intracytoplasmic motifs) or with $1 \%$ Triton X-100 (for nuclear motifs) in PBS. Tissue sections were blocked for 1 hour in $4 \%$ FBS and $1 \%$ bovine serum albumine (BSA) or in 5\% BSA in PBS. If the primary antibody was produced in mouse, the M.O.M.TM Immunodetection Kit (Vector Lab) was applied to enable blocking of endogenous Fc receptors that could be recognized by the secondary antibody. Incubation with primary antibody was performed overnight at $4^{\circ} \mathrm{C}$ in a humidified chamber. Following the primary antibody incubation, sections were washed in PBS and then incubated with the secondary antibody (RT) during 1 hour. In order to amplify the fluorescence intensity of several antibodies, two streptavidin conjugated fluorophores were used: Streptavidin 
conjugated with Alexa 555 (S32355, Invitrogen), at 1:500 dilution and Streptavidin conjugated with Allophycocyanin (APC) (SA1005, Life Technologies), at 1:100 dilution. After washing, sections were mounted with FluoroshieldTM (F6182, Sigma-Aldrich), Vectashield mounting media with DAPI (H-1200, Vector) and observed/photographed either in a Zeiss Axiovert 200M inverted fluorescence microscope, in a Nikon eclipse 90i fluorescence microscope, in a Leica TCS SP5 laser scanning confocal microscope or in GE IN Cell Analyzer 2000 High-Content Screening microscope.

Cytospin immunofluorescence was performed following heart digestion (see neonatal cardiomyocyte isolation section). Isolated cardiac cells were plated onto glass slides using cytospin (Shandon Cytospin ${ }^{\circledR} 4$, Thermo Scientific) and spun for $5 \mathrm{~min}$ at $130 \mathrm{~g}$. Cells were permeabilized with $0.5 \%$ Triton for $7 \mathrm{~min}$ and washed three times in PBS with $0.2 \%$ Tween. Incubation with the primary antibody was carried out for 2 hours and the secondary antibody incubation for 30min, in the dark. Finally, nuclei were stained with DAPI for $10 \mathrm{~min}$ and slides mounted in a PBS glycerol solution (1:9) with $2.5 \% \mathrm{n}$-propyl-gallate (SigmaAldrich) without DAPI.

List of primary antibodies used and specification of the working dilution

\begin{tabular}{|c|c|c|}
\hline Antibody & Dilution & Reference \\
\hline $\begin{array}{l}\text { Sarcomeric- } \alpha \text {-Actinin (Mouse } \\
\qquad \lg G)\end{array}$ & $1: 400$ & A7811, Sigma \\
\hline Vimentin (Mouse IgG1/K) & $1: 50$ & MS-190-P, Thermo \\
\hline CD31 (Goat IgG) & $\begin{array}{c}\text { 1:250 (requires HIER with Tris- } \\
\text { EDTA, pH 9.0) }\end{array}$ & $\begin{array}{l}\text { sc-1506, Santa Cruz } \\
\text { Biotechnology }\end{array}$ \\
\hline CD45 (Goat IgG) & $1: 100$ & AF114, R\&D \\
\hline $\begin{array}{l}\text { Alpha Smooth Muscle Actin } \\
\text { (Mouse } \lg \text { ) }\end{array}$ & $1: 400$ & A5228, Sigma \\
\hline PH3 (Rabbit lgG) & $1: 800$ & \#3377, Cell Signalling \\
\hline Fibronectin (Rabbit IgG) & $1: 400$ & F-3648, Sigma \\
\hline Laminin (Rabbit IgG) & $1: 400$ & L9393, Sigma \\
\hline Tenascin-C (Rat IgG) & $1: 100$ & LAT2, gift A. Sonnenberg \\
\hline CD29 (Rat IgG) & $1: 50$ & 14-0292, eBioscience \\
\hline Collagen IV (Goat IgG) & $1: 100$ & AB769, Chemicon \\
\hline Aurora-B (Mouse IgG1) & $\begin{array}{l}\text { 1:50 (requires HIER with } \\
\text { sodium citrate acid buffer) }\end{array}$ & $\begin{array}{c}\text { 611082, BD Transduction } \\
\text { Laboratories }\end{array}$ \\
\hline $\begin{array}{l}\text { Cardiac Troponin I (Rabbit } \\
\text { IgG) }\end{array}$ & $\begin{array}{l}\text { 1:200 (requires HIER with } \\
\text { sodium citrate acid buffer) }\end{array}$ & ab47003, Abcam \\
\hline PCM-1 (Rabbit Polyclonal) & $1: 500$ & HPA023370, Sigma \\
\hline
\end{tabular}


List of secondary antibodies used and specification of the working dilution

\begin{tabular}{|c|c|c|}
\hline Antibody & Dilution & Reference \\
\hline Alexa Fluor 488 Donkey anti Mouse IgG & $1: 1000$ & A-21202, Invitrogen \\
\hline Alexa Fluor 594 Donkey anti Mouse IgG & $1: 1000$ & A-21203, Invitrogen \\
\hline Alexa Fluor 488 Goat anti Mouse IgG & $1: 1000$ & A11017, Molecular Probes \\
\hline Cy3 Goat anti Mouse IgG & $1: 200$ & $115-165-003$, Jackson \\
\hline Alexa Fluor 488 Goat anti Rabbit & $1: 200$ & $111-545-003$, Jackson \\
\hline Alexa Fluor 488 Donkey anti Rabbit IgG & $1: 1000$ & A-11055, Invitrogen \\
\hline Alexa Fluor 568 Donkey anti Rabbit IgG & $1: 1000$ & A-10042, Invitrogen \\
\hline Alexa Fluor 633 Goat anti Rabbit IgG & $1: 1000$ & A21070, Molecular Probes \\
\hline Biotinylated Donkey anti Rabbit IgG & $1: 250$ & A16033, Life Technologies \\
\hline Alexa Fluor 568 Donkey anti Goat IgG & $1: 1000$ & A-11057, Invitrogen \\
\hline Biotinylated Donkey anti Goat IgG & $1: 250$ & A16009, Life Technologies \\
\hline Alexa Fluor 488 Donkey anti Rat IgG & $1: 1000$ & A-21208, Invitrogen \\
\hline Alexa Fluor 568 Goat anti Rat IgG & $1: 1000$ & A11077, Molecular Probes \\
\hline M.O.M. Biotinylated anti mouse Ig & $1: 250$ & MKB-2225, Vector Laboratories \\
\hline Biotinylated Goat anti Rat IgG & $1: 250$ & BA-9400, Vector Laboratories \\
\hline
\end{tabular}

\section{$\underline{\text { High Content Screening (HCS) }}$}

The proliferative response of neonatal murine hearts at $7 \mathrm{~d}$ injury and (neo)vascularization at 60d injury were evaluated using IN Cell Analyzer 2000 (GE Health Care Life Sciences) with assistance of IN Cell Developer software (GE Health Care Life Sciences). This microscope allowed a complete scan of the sample and its software was used to quantify, through overlapping regions of interest (ROIs), the total number of proliferating cardiomyocytes (colocalization of DAPI, PH3 and sarcomeric- $\alpha$-Actinin expression) or the total number of endothelial cells (colocalization of DAPI and CD31 expression) per area (quantified in advance by the assembly of individual images in FIJI v2.0.0-rc-54/.51h.).

Flow Cytometric profile of Cardiac Populations and Fluorescence Activated Cell Sorting (FACS)

Flow cytometry was performed to characterize cardiac stroma, namely fibroblast populations, from E17 to adulthood and inflammatory populations $2 \mathrm{~d}, 5 \mathrm{~d}$ and $7 \mathrm{~d}$ postsurgery. Cardiac cells were isolated by digestion of cardiac tissue fragments with crude collagenase (C2139, Sigma-Aldrich ${ }^{\circledR}$ ) at $200 \mu \mathrm{g} / \mathrm{ml}$ concentration and DNase (A3778, VWR) at $60 \mathrm{U} / \mathrm{ml}$ in Hank's balanced salt solution (HBSS) (H9269, Sigma-Aldrich ${ }^{\circledR}$ ). Collagenase digestions were performed during $15 \mathrm{~min}$ at $37^{\circ} \mathrm{C}$ until no tissue was observed by visual inspection. After each digestion, the suspension was decanted, the medium collected (cellular portion) and a new collagenase/DNase solution was added to the remaining tissue fragments. The collected cell suspension was mixed with HBSS with $10 \%$ FBS to neutralize enzymatic activity and was kept on ice. The collected cellular fraction of each digestion was 
combined and washed in FACS medium ( $0.01 \%$ sodium azide and $3 \%$ FBS in PBS). Cells were evenly distributed for each staining in a round bottom multiwell plate. Fc receptors were blocked using anti-mouse CD16/CD32 at $0.5 \mu \mathrm{g} / \mu \mathrm{l}$ in FACS medium during $20 \mathrm{~min}$. After two washes in FACS media, cells were incubated during $30 \mathrm{~min}$ with the antibody cocktail on ice and in the dark. Cells were washed twice in FACS media and transferred to FACS tubes. In order to exclude nonviable cells from the analysis, $0.5 \%$ of propidium iodide ( $\mathrm{PI}$ ) ( $\mathrm{P} 4170$, Sigma-Aldrich) was added to the cell suspension 1-2 min prior to analysis. Fifty thousand events (of appropriate size and complexity) per staining were acquired in the cytometer FACS Canto II (BD Biosciences). For characterization of inflammatory cells, after antibody incubation, cells were fixed in 1\% PFA in FACS medium during $15 \mathrm{~min}$ and acquired afterwards. Subsequent analysis and graphing were performed in FlowJo VX software.

List of antibodies and isotype controls used in flow cytometry and specifications of the working dilution

\begin{tabular}{|c|c|c|}
\hline Antibody & Dilution & Reference \\
\hline CD31-PeCy7 (Rat IgG2a) & $1: 100$ & 25-0311-81, eBioscience \\
\hline CD45-PeCy7 (Rat IgG2b) & $1: 100$ & $25-0451-81$, eBioscience \\
\hline TER119-PeCy7 (Rat IgG2b) & $1: 100$ & 116222, Biolegend \\
\hline CD90.2-FITC (Rat IgG2b) & $1: 100$ & 105306, Biolegend \\
\hline CD90.2 PE (Rat IgG2a) & $1: 100$ & 553930, BD Pharmingen \\
\hline CD140a-APC (FL4) (Rat IgG2a) & $1: 100$ & 135907, Biolegend \\
\hline SCA-1 FITC (Rat IgG2a) & $1: 100$ & $11-5981-81$, eBioscience \\
\hline SCA-1-PE (FL1) (Rat IgG2a) & $1: 100$ & $12-5981-81$, eBioscience \\
\hline CD44-APC/Cy7 (Rat IgG2b) & $1: 50$ & 103028, Biolegend \\
\hline CD45-PE (Rat IgG2b) & $1: 100$ & $12-0451-82$, eBioscience \\
\hline CD11b-APC (Rat / IgG2b) & $1: 100$ & $17-0112-82$, eBioscience \\
\hline F4/80-FITC (Rat / IgG2a) & $1: 100$ & $11-4801-82$, eBioscience \\
\hline Ly6C-PeCy7 (Rat / IgG2c) & $1: 100$ & $25-5932-82$, eBioscience \\
\hline Gr-1-FITC (Rat IgG2b) & $1: 100$ & $11-5931-82$, eBioscience \\
\hline CD3e-PacBlue (S. Hamster IgG2) & $1: 100$ & 558214, BD Biosciences \\
\hline CD45R-PeCy7 (Rat IgG2a) & $1: 100$ & $25-0452-82$, eBioscience \\
\hline Isotype Control PeCy7 (Rat IgG2a) & $1: 100$ & 400521, Biolegend \\
\hline Isotype Control PeCy7 (Rat IgG2b) & $1: 100$ & 400617, Biolegend \\
\hline Isotype Control FITC (Rat IgG2a) & $1: 100$ & 400505, Biolegend \\
\hline Isotype Control FITC (Rat IgG2b) & $1: 100$ & IC013F, R\&D \\
\hline Isotype Control PE (Rat IgG2a) & $1: 100$ & 400508, Biolegend \\
\hline Isotype Control APC (Rat IgG2a) & $1: 100$ & IC006A, R\&D \\
\hline Isotype Control APCCy7 (Rat IgG2b) & $1: 100$ & 400623, Biolegend \\
\hline & & \\
\hline
\end{tabular}


Real-time Polymerase Chain Reaction (qRT-PCR)

Primer sequences for qRT-PCR

\begin{tabular}{|c|c|c|c|}
\hline Gene & Primer Sequence (5' to 3') & PCR Product Length & $\begin{array}{c}\text { Annealing } \\
\text { Temperature ( }\end{array}$ \\
\hline Gapdh
\end{tabular}

\section{$\underline{\text { Neonatal Cardiomyocyte Isolation }}$}

Seven days post-surgery neonatal hearts were harvested and cut into small fragments (approximately $2 \mathrm{~mm}$ ), before being flash frozen in liquid nitrogen until further use. Then, fragments were thawed at RT and fixed with 4\% PFA in PBS for 2 hours with stirring (100 rpm). Following fixation, fragments were digested with collagenase type II (Worthington, CLS-2) ( $3 \mathrm{mg} / \mathrm{ml}$ in HBSS) overnight at $37^{\circ} \mathrm{C}$ (100 rpm) and mechanically dissociated until no fragments were detected (additional digestions were sometimes required, depending on the original size of the fragment). In order to inactivate collagenase, equal volume of HBSS 
with $10 \%$ FBS was added. At this stage, cells can be stored at $4^{\circ} \mathrm{C}$ or, alternatively, processed for cytospin immunofluorescence or for imaging flow cytometry (see Imaging Flow Cytometry). This methodology yields $\sim 70 \%$ purity of $\mathrm{CMs}$.

\section{Imaging Flow Cytometry}

Aiming at performing a morphometric characterization of cardiomyocytes, cell suspensions were centrifuged at $300 \mathrm{~g}$ for $10 \mathrm{~min}$ and the cellular portion was then resuspended in FACS medium $(0.01 \%$ sodium azide and $3 \% \mathrm{FBS}$ in PBS) and permeabilized with $1 \mathrm{X}$ BD Perm/Wash ${ }^{\mathrm{TM}}$ Buffer during $15 \mathrm{~min}$ at RT. Incubation with primary and secondary antibodies lasted for 2 hours and 30 min on ice, respectively. Both antibodies were diluted in $1 X$ BD Perm/Wash ${ }^{\mathrm{TM}}$ Buffer and incubations were separated by two washes with $1 \mathrm{X} B D$ Perm $/ \mathrm{Wash}^{\mathrm{TM}}$ Buffer at $800 \mathrm{~g}$. Finally, they were ressuspended in PBS and kept at $4^{\circ} \mathrm{C}$ until acquisition. Immediately before acquiring in Imagestream ${ }^{\circledR} X$, cells were filtered (100 $\mu \mathrm{m}$ cell strainer) and their nuclei were stained with $200 \mu \mathrm{M}$ of DRAQ5 (5 mM, Biostatus).

Cardiomyocyte morphometric analysis was performed using IDEAS ${ }^{\circledR}$ software. This software calculates several features of CMs (area, major axis, minor axis and aspect ratio) and allows mask creation (definition of a specific area), which allows the assessment of nuclear number. Unfocused cells were promptly removed by eliminating cells with Gradient RMS smaller than 40 and debris and duplets were excluded by removing objects with a small area and high aspect ratio. Whenever the aspect ratio was smaller than 0.55 , cardiomyocytes were classified as "Rod" and above this value were considered "Round".

\section{Adult Cardiomyocyte Isolation}

Adult cardiomyocytes were isolated by reperfusion of the heart in a Langendorff system with liberase TM (05401127001, Roche) at $13.3 \mu \mathrm{g} / \mathrm{ml}$ concentration and trypsin (27250018, Gibco) at $13.8 \mu \mathrm{g} / \mathrm{ml}$ concentration in a heated aqueous buffer containing: $\mathrm{NaCl}$ (113mM), $\mathrm{KCl}(4.7 \mathrm{mM}), \mathrm{MgSO} 4(1.2 \mathrm{mM}), \mathrm{Na} 2 \mathrm{HPO} 4$ (0.6mM), KH2PO4 (0.6mM), NaHCO3 $(12 \mathrm{mM}), \mathrm{KHCO} 3(10 \mathrm{mM})$ and Taurine $(30 \mathrm{mM})$. Perfusion was performed under physiological pressure $( \pm 80 \mathrm{mmHg}$ ) and rate $(3 \mathrm{ml} / \mathrm{min})$ and lasted for 25 to $30 \mathrm{~min}$. At this point the heart appears swollen, pale and flaccid and is cut into small pieces, which are further dissociated by a plastic pipette. Digestion is halted by adding ice-chilled FBS to the buffer (final concentration 10\%) and the cell suspension is filtered using a $300 \mu \mathrm{m}$ mesh. Then CMs are fixed for 30 min with $4 \%$ PFA in PBS and stored at $4^{\circ} \mathrm{C}$ in PBS with $0,1 \%$ sodium azide.

The area of adult CMs, isolated from $60 \mathrm{~d}$ and $180 \mathrm{~d}$ post-surgery hearts, was evaluated by immunofluorescence in cytospins.

The volume of adult CMs, isolated from $60 \mathrm{~d}$ post-surgery hearts, was evaluated with the assistance of IMARIS 8.4.1. The surface module allowed a semi-automatic segmentation of $\mathrm{CMs}$ and volume determination. 



\section{CHAPTER 4}

\section{Stereological estimation of cardiomyocyte number and proliferation}

Vasco Sampaio-Pinto ${ }^{a, b, c, d, e}$, Elsa D Silva ${ }^{a, b}$, Tiago L Laundos ${ }^{a, b, c}$, Paula da Costa Martins ${ }^{d, e, f}$, Perpétua Pinto-do-Óa,b,c, Diana S Nascimento ${ }^{a, b, c}$

a i3S - Instituto de Investigação e Inovação em Saúde, Universidade do Porto, Porto, Portugal;

b INEB - Instituto Nacional de Engenharia Biomédica, Universidade do Porto, Porto, Portugal;

' ICBAS - Instituto de Ciências Biomédicas Abel Salazar, Universidade do Porto, Portugal;

${ }^{d}$ Department of Cardiology, CARIM School for Cardiovascular Diseases, Faculty of Health, Medicine and Life Sciences, Maastricht University, Maastricht, The Netherlands;

e Department of Molecular Genetics, Faculty of Sciences and Engineering, Maastricht University, Maastricht, The Netherlands;

${ }^{f}$ Department of Physiology and Cardiothoracic Surgery, Faculty of Medicine, University of Porto, Porto, Portugal.

Methods. 2020; S1046-2023(19)30312-3

doi: 10.1016/j.ymeth.2020.06.002 


\begin{abstract}
Cardiovascular diseases remain the leading cause of death, largely due to the limited regenerative capacity of the adult mammalian heart. Yet, neonatal mammals were shown to regenerate the myocardium after injury by increasing the proliferation of pre-existing cardiomyocytes. Re-activation of cardiomyocyte proliferation in adulthood has been considered a promising strategy to improve cardiac response to injury. Notwithstanding, quantification of cardiomyocyte proliferation, which occurs at a very low rate, is hampered by inefficient or unreliable techniques.

Herein, we propose an optimized protocol to unequivocally assess cardiomyocyte proliferation and/or cardiomyocyte number in the myocardium. Resorting to a stereological approach we estimate the number of cardiomyocytes using representative thick sections of left ventricle fragments. This protocol overcomes the need for spatial-temporal capture of cardiomyocyte proliferation events by focusing instead on the quantification of the outcome of this process. In addition, assessment of cardiomyocyte nucleation avoids overestimation of cardiomyocyte proliferation due to increased binucleation.

By applying this protocol, we were able to previously show that apical resection triggers proliferation of pre-existing cardiomyocytes generating hearts with more cardiomyocytes. Likewise, the protocol will be useful for any study aiming at evaluating the impact of neomyogenic therapies.
\end{abstract}




\section{Introduction}

Cardiomyocytes are the contractile unit of the heart responsible for generating forces required to circulate the blood through the vascular system. Such strong specialization is often associated with low cellular turnover and, in fact, postnatal cardiomyocyte renewal is very scarce [1]. As a consequence, cardiomyocyte loss or dysfunction frequently leads to heart failure [2]. For this reason, several therapeutic strategies are currently under investigation to preserve and/or restore the myocardium, namely involving tissue engineering [3-5], cellular-based therapies [6, 7] and/or administration of factors or molecules promoting neomyogenesis $[8,9]$.

Mammals were shown to display transient cardiac regenerative capacity around birth [10, 11 ] by increasing proliferative rates of pre-existing cardiomyocytes [12, 13]. Significant effort has been applied to understand the molecular, cellular and microenvironmental factors regulating the proliferation of cardiomyocytes in the neonate, towards their activation in disease during adulthood.

Shortly after birth, mammalian hearts undergo maturation, characterized by a profound physiologic and metabolic remodeling (reviewed in [14]). During this process, most cardiomyocytes become multinucleated or polyploid due to incomplete karyokinesis or cytokinesis, respectively [15-17]. Overall, this maturation is thought to render adult cardiomyocytes to a post-mitotic arrest. As importantly discussed by Zebrowski and colleagues, ex vivo quantification of cardiomyocyte proliferation is technically challenging as both lineage tracing methods and expression of cell cycle markers do not accurately predict whether a cardiomyocyte will divide or binucleate [18]. Application of these strategies in thin sections can be even more misleading as cardiomyocytes are often sliced, hindering complete tridimensional imaging of the cell. Disregarding these limitations, cardiomyocyte proliferation is still typically addressed by detection of DNA synthesis (e.g. Bromodeoxyuridine (BrdU) or 5-Ethynyl-2'-deoxyuridine (EdU) incorporation) and/or expression of cell cycle markers (e.g. Ki67, pH3, Aurora-B) in cardiomyocytes on tissuesections.

Herein, we describe a protocol to quantify cardiomyocyte proliferation and total cardiomyocyte number in the heart by combining the power of 3D-imaging with stereology and surpassing the limitations of traditional methods in use.

\section{Rationale}

Stereology consists on the tridimensional interpretation of two-dimensional sections of tissues. In order to estimate the number of cardiomyocytes in the left ventricle (LV), we have to determine (1) the number of cardiomyocytes in a known myocardial volume, sampled from the LV and (2) the total LV myocardial volume. Because it is not possible to extrapolate the 2 factors in the same heart owing to technical variations, two groups of hearts from the same condition are used (Figure 1). 


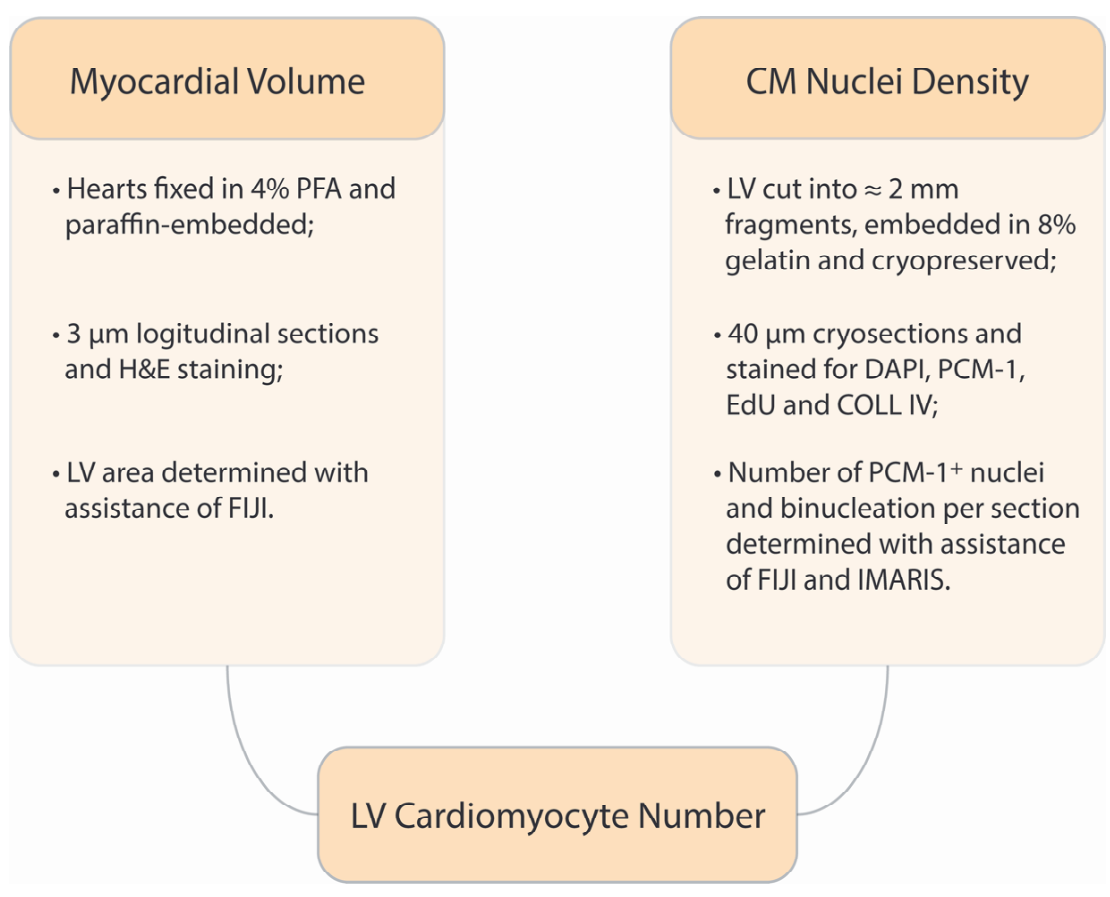

Figure 1 - Schematic diagram of heart processing for quantification of myocardial volume (left panel) and cardiomyocyte (CM) nuclei density (right panel).

\section{Reagents \& Equipment}

$\mathrm{KCl}$ - Sigma-Aldrich;

10\% Formalin neutral buffer - VWR BDH \& Prolabo;

Absolute, $96 \%$, 70\% ethanol - Aga;

Clear Rite - Richard-Allan Scientific;

Shandon Histoplast - Thermo Fisher Scientific;

Hematoxylin - Klinipath;

1\% Eosin Y (Aqueous) - Clintech;

Trichrome (Masson) Stain kit - Sigma-Aldrich;

Bouin's Fixative - VWR;

Xylene - VWR;

DPX mountant for histology - Sigma -Aldrich;

Gelatin - Merck;

Isopentane/2-Methylbutane - VWR;

$37 \%$ Formaldehyde - Sigma-Aldrich;

Click-iт ${ }^{\mathrm{TM}}$ Plus EdU Cell Proliferation Kit for Imaging, Alexa Fluor ${ }^{\mathrm{TM}} 647$ dye - Invitrogen (C10640);

Triton X-100 - Sigma-Aldrich;

Anti-PCM-1 Antibody (Rabbit polyclonal) - Sigma-Aldrich (HPA023370);

Anti-Collagen IV Antibody (Goat IgG) - Chemicon (AB769);

Alexa Fluor 488 Donkey anti Rabbit IgG - Invitrogen (A-11055); 
Alexa Fluor 568 Donkey anti Goat IgG - Invitrogen (A-11057);

DAPI - Sigma-Aldrich (D9542);

Fluoroshield histology mounting medium - Sigma-Aldrich (F6182);

Peel-a-way embedding molds - Sigma-Aldrich;

Superfrost Plus Microscope Slides - VWR;

Ultra-Low Temperature Freezer - Eppendorf;

Cryostat - Thermo Scientific (Microtom HM 550);

Microtome - Leica (RM2255);

Digital slide scanner - Hamamatsu (Nanozoomer 2.0-HT);

Confocal Microscope - Leica (TCS SP5).

Working solutions were prepared by dissolving powered reagents or diluting stock solutions in deionized water.

\section{Software}

FIJI - Open source imaging software: version 2.0.0-rc-69/1.52p [19];

IMARIS - Oxford Instruments plc, Oxon, UK. Software available at: https://imaris.oxinst.com;

Huygens - Scientific Volume Imaging B.V., Hilversum, The Netherlands. Software available at: http://svi.nl.

Note: DeconvolutionLab2 [20] can be used as an open-source alternative to Huygens for the deconvolution of images obtained with the confocal microscope.

\section{Experimental Procedures}

\section{Animals and Apical Resection Injury Model}

All animal work was approved by the IBMC-INEB (Instituto de Biologia Molecular e CelularInstituto de Engenharia Biomédica) Animal Ethics Committee, and by the Direcção Geral de Veterinária (permit 022793), and is in conformity with Directive 2010/63/EU. Humane endpoints were followed in accordance with the OECD Guidance Document on the Recognition, Assessment, and Use of Clinical Signs as Humane Endpoints for Experimental Animals Used in Safety Evaluation. Apical resection was performed as described in [11] in male and female littermates to minimize interindividual variation. Hence, all analysis conducted in our study were obtained in mixed groups. Importantly, sex-related differences can be scrutinized by separately analyzing male and female responses to a treatment (i.e. cardiac injury, neomyogenic therapy, etc.).

\section{EdU Pulse-Chase Assay}

To permanently label replicating cells, animals were injected subcutaneously (s.c.) with 25 $\mu \mathrm{g}$ of EdU per gram of body weight, using a $2.5 \mu \mathrm{g} / \mu \mathrm{l}$ working solution of EdU in sterile 
phosphate-buffered saline (PBS). As EdU labeling diminishes at each cell division, the concentration and frequency of administration depends on the expected replication rate and may require optimization (as described in [21] for the dentate gyrus). In our setting, EdU was administered several times, adjusting the volume injected to the current weight of the animals. This approach ensured an efficient labeling of newly dividing cells while preserving the EdU signal on cells that underwent replication at an earlier time point. As described previously by our group [11], for an in vivo study lasting for 15 days post-birth, animals received 3 s.c. injections of EdU (i.e. immediately after apical resection/shamsurgery at postnatal day 1 (P1), at P6 and P8). EdU incorporation was detected using the Click-iT EdU reaction cocktail, in accordance to manufacturer's instructions (see immunohistochemistry section for further details).

\section{Myocardial Volume Determination}

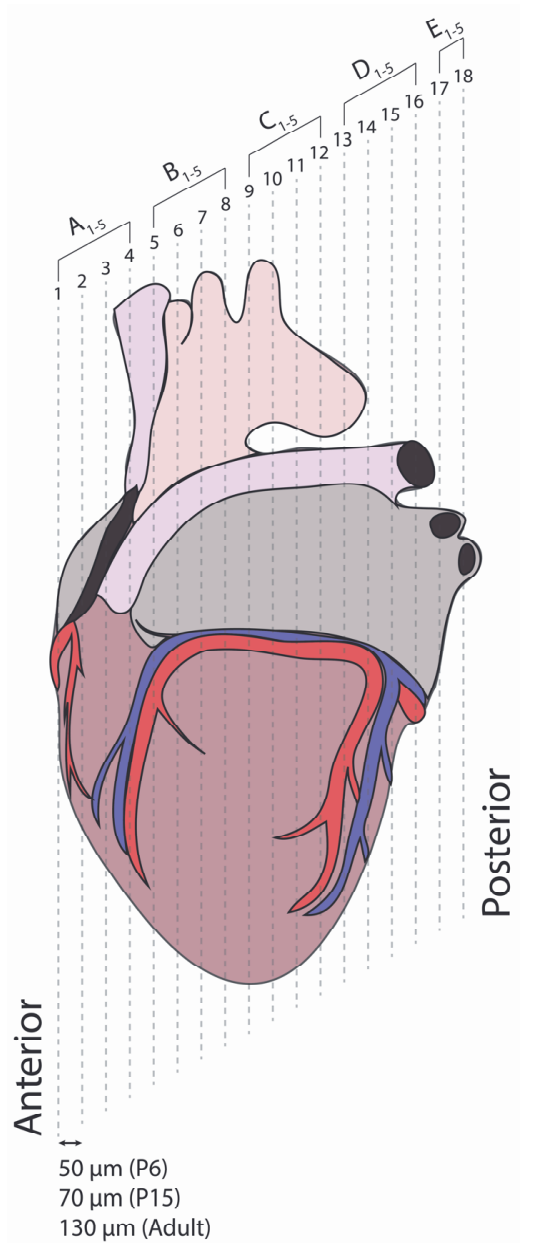

Figure 2 - Schematic representation of heart sectioning for assessment of myocardial volume. Trimming distances were adjusted to heart size (i.e. $50 \mu \mathrm{m}, 70 \mu \mathrm{m}$ and $130 \mu \mathrm{m}$ for P6, P15 and adult hearts respectively) yielding approximately the same number of sections.

\section{Sample preparation}

Immediately after necropsy, hearts were injected with $50-100 \mu$ of $4 \mathrm{M} \mathrm{KCl}$ in the LV for cardioplegia. Hearts were placed in a Petri dish containing PBS and associated vessels and 
fat were removed using a scalpel. Hearts were photographed and fixed in $4 \%$ formalin neutral buffer for $24 \mathrm{~h}$, at $4^{\circ} \mathrm{C}$. For paraffin embedding, hearts were processed during 12 hours through increasing series of ethanol concentrations, Clear Rite and paraffin at $56^{\circ} \mathrm{C}$. Hearts were then included in paraffin blocks and sectioned longitudinally into $3 \mu \mathrm{m}$ sections (Figure 2).

\section{Heart sampling - sectioning}

Paraffin-embedded hearts were sectioned into $3 \mu \mathrm{m}$ sections and 5 replicate slides were performed (Figure 2 and Figure 3 - A1-A5; B1-B5, etc.). In order to obtain representative samples of the hearts, consecutive sections were placed in the same position in different glass slides. After each series of 5 consecutive sections, the heart was trimmed (depending on the size of the heart/ontogenic time point; in our setting: $50 \mu \mathrm{m}$ for P6 hearts, $70 \mu \mathrm{m}$ for P15 hearts and $130 \mu \mathrm{m}$ for adult hearts). The same procedure was repeated until the heart was fully sectioned from anterior to posterior side (Figure 2). Then, sections were dried in an oven at $37^{\circ} \mathrm{C}$. In order to assess the LV volume, heart sections were stained with a modified Masson's Trichrome stain (Hematoxilin \& Eosin (H\&E) stain can be used in alternative).
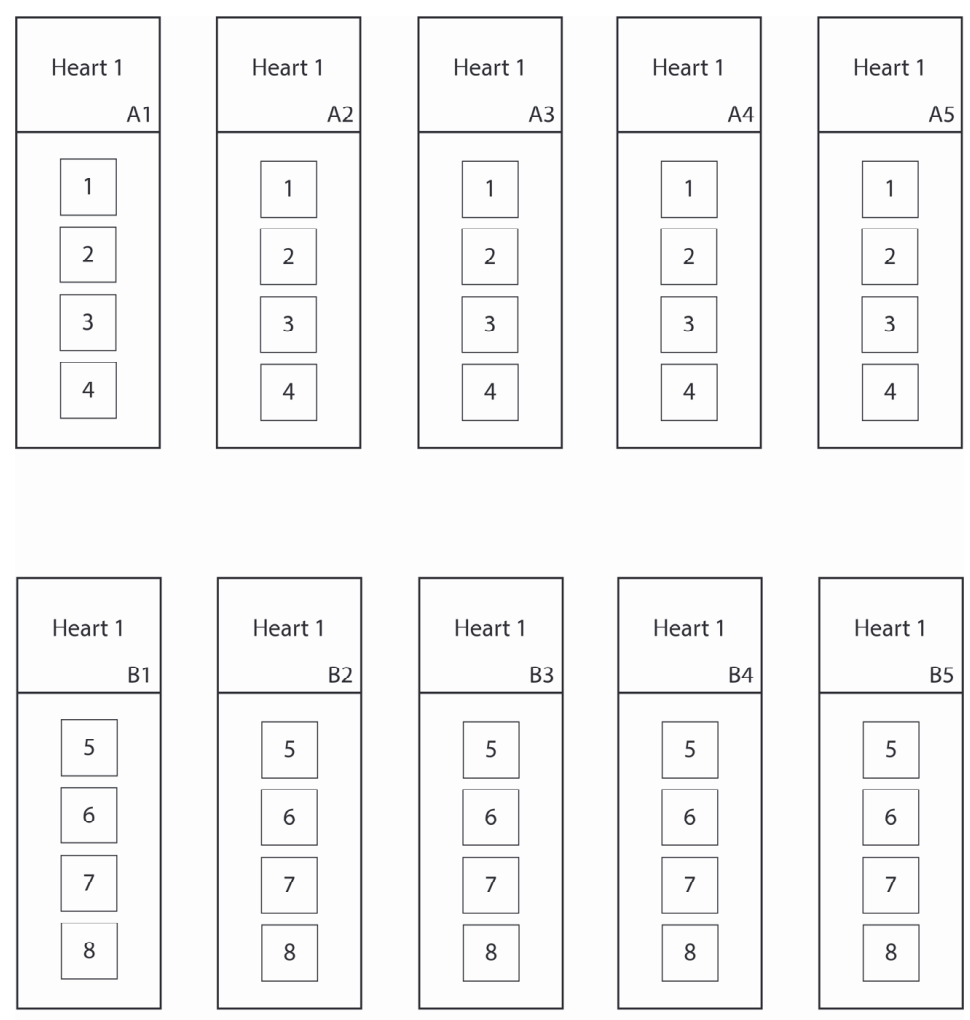

$\cdots$

Figure 3 - Schematic diagram of section positioning in histological slides, ensuring unbiased and representative sectioning of the hearts. Five replicates were generated from the same region of the heart (series $A$, series $B, \ldots$..). 


\section{Modified Masson's Trichrome stain}

Sections were deparaffinized by submersion in xylene twice during $10 \mathrm{~min}$ and hydrated in decreasing ethanol series $(100 \% \rightarrow 96 \% \rightarrow 70 \% \rightarrow 50 \% \rightarrow \mathrm{dH} 2 \mathrm{O})$. Nuclei were pre-stained with Celestine blue for $5 \mathrm{~min}$ and hematoxylin for $5 \mathrm{~min}$. Sections were submerged in tap water for 2 min to allow bluing of the nuclei and rinsed in $\mathrm{dH} 2 \mathrm{O}$ before fixation in Bouin solution for 1 hour. Remaining procedures were performed according to Trichrome (Masson) stain kit instructions. Sections were diafanized in xylene and mounted in DPX mountant for histology, covered with a coverslip and left to dry overnight horizontally at room temperature (RT) before image acquisition.

\section{Hematoxylin \& Eosin stain}

Sections were deparaffinized by submersion in xylene twice during $10 \mathrm{~min}$ and hydrated in decreasing ethanol series $(100 \% \rightarrow 96 \% \rightarrow 70 \% \rightarrow 50 \% \rightarrow d H 2 O)$. Heart sections were then incubated with hematoxylin for $5 \mathrm{~min}$, submerged in tap water for $2 \mathrm{~min}$ to allow bluing of nuclei and rinsed in $\mathrm{dH} 2 \mathrm{O}$. Immediately after, sections were submerged in aqueous $1 \%$ Eosin for $5 \mathrm{~min}$ and passed through an increasingly concentrated series of ethanol to dehydrate and, finally, were diaphanized by 3 submersions of $5 \mathrm{~min}$ in xylene. DPX mountant for histology was applied to the sections, covered with a coverslip and the mounted slides were left to dry overnight in horizontal position at RT prior to image acquisition.

\section{Morphometric analysis}

After image acquisition, the LV area of the heart was determined with assistance of FIJI. Upon defining the scale, the polygon tool was used to create selections delineating the LV and the LV chamber (when present). After measuring both selections, LV area was determined by subtracting the chamber area (Figure 4A - yellow) to the LV area (Figure 4A - green). The same procedure was performed for all the sections of the heart. To determine the LV volume, the sum of all LV areas was multiplied by the distance separating adjacent sections.

Since formalin-fixed paraffin-embedded (FFPE) tissues shrink [22, 23], LV volume requires correction. For that, the long-axis and short-axis length of freshly isolated hearts (measured in images of freshly isolated hearts) was compared to the long-axis and short-axis length of FFPE Masson's Trichrome or H\&E stained hearts (Figure 4B). In our setting, the long-axis and short-axis exhibited an average length reduction of $11.8 \%$ after fixation and embedding. Hence, the LV volume estimated before was divided by $0.882(100 \%-11.8 \%=$ $88.2 \%)$ to account for this shrinkage.

Note: estimation of LV volume assumes that the distance between representative sections is constant and therefore thickness of sections and trimming distances must be strictly controlled and followed. 
A
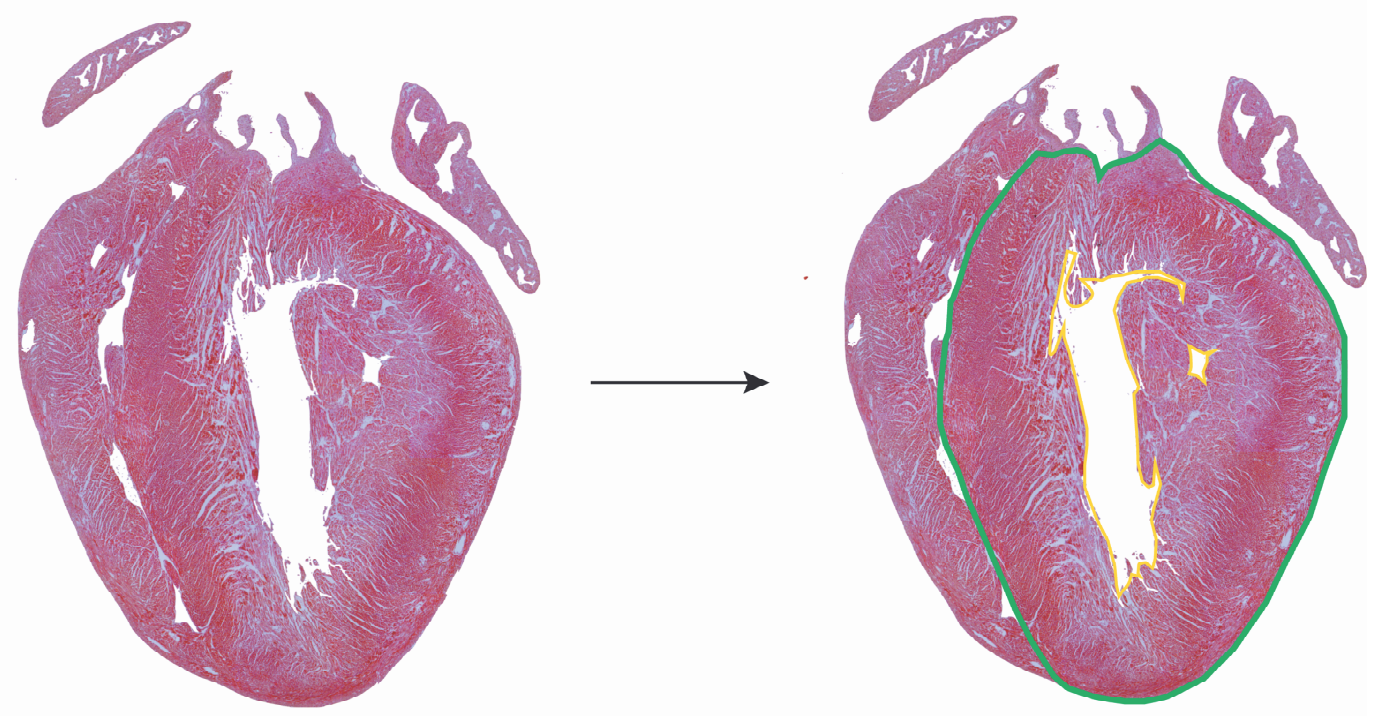

$\mathrm{LV}$ area $=$ Green area - Yellow area

B
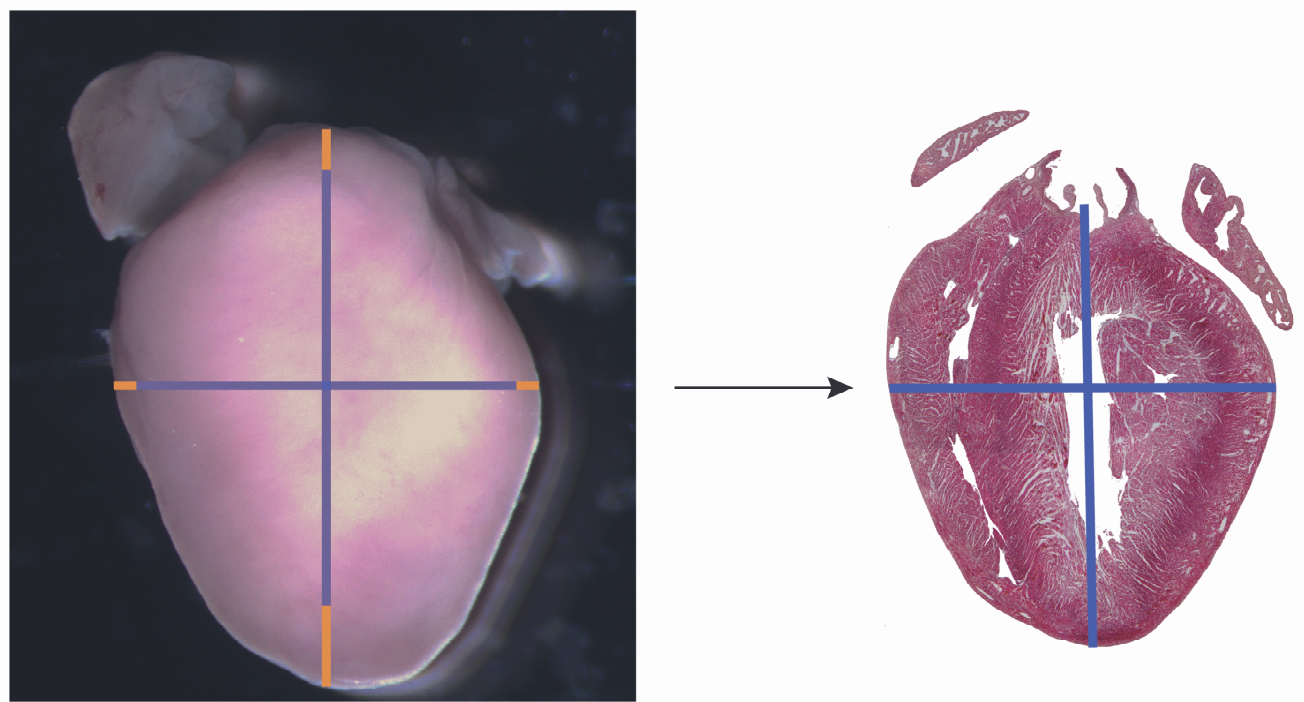

\% Shrinkage: (1 - Ratio Blue/Orange Segment) x 100

Figure 4 - A - Illustration of the methodology employed to measure LV area (subtraction of yellow area (LV chamber) to the green area (whole LV). B - Illustration of the methodology employed to determine heart shortening during formalin-fixation and paraffin embedding (average of the ratios between blue and orange segments). 


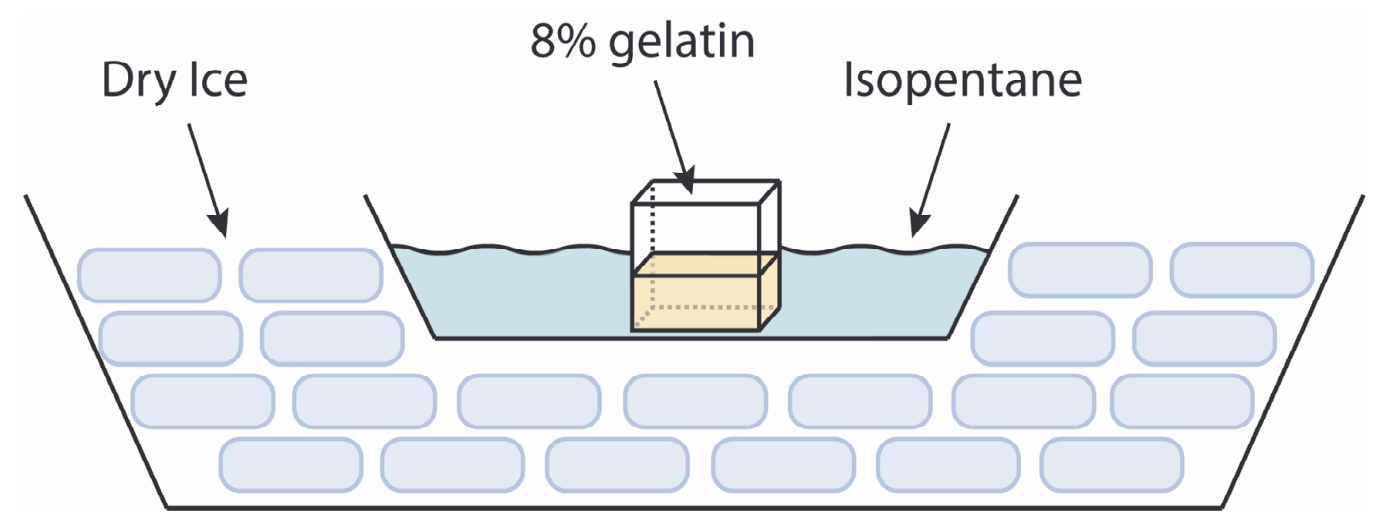

Figure 5 - Schematic diagram of the procedure applied to cryopreserve the heart fragments used to assess cardiomyocyte nuclei density.

\section{Determination of Cardiomyocyte Number}

\section{Sample preparation}

Immediately after necropsy, hearts were injected with $50-100 \mu \mathrm{l}$ of $4 \mathrm{M} \mathrm{KCl}$ in the LV for cardioplegia. Hearts were placed in a Petri dish containing PBS, the LV was isolated and cut into 1-2 mm pieces using a scalpel. Further segmentation of the LV (e.g. apical and remote myocardium) can be performed to address a particular research question. LV fragments were transferred to a peel-a-way embedding mold containing pre-warmed $8 \%$ gelatin in PBS at $37^{\circ} \mathrm{C}$. Tissue was frozen using dry-ice chilled isopentane and stored at $-80^{\circ} \mathrm{C}$ until further use (Figure 5). Forty $\mu \mathrm{m}$ sections were prepared using a cryostat and placed on positively charged glass slides (e.g. Superfrost Plus microscope slides). Sections were allowed to dry for $30 \mathrm{~min}$ at RT and stored at $-80^{\circ} \mathrm{C}$. Five replicate slides were prepared with two sections containing multiple fragments from the same heart (Figure 6).

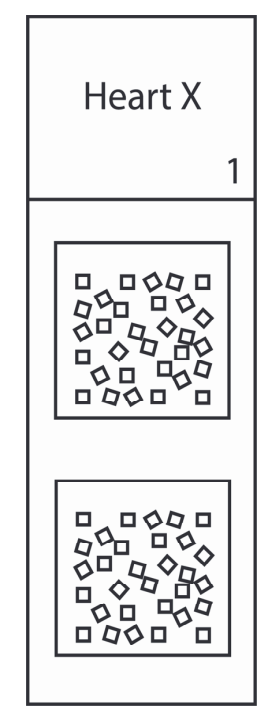

Figure 6 - Forty $\mu \mathrm{m}$ cryosections of heart fragments were obtained and organized in histological slides as represented in the image. 


\section{Immunohistochemistry}

Cryosections were removed from $-80^{\circ} \mathrm{C}$ and incubated for $40 \mathrm{~min}$ in pre-heated PBS at $37^{\circ} \mathrm{C}$ to remove the gelatin. Tissue was fixed using $4 \%$ formaldehyde in PBS for 15 min at RT, washed twice in $3 \%$ bovine serum albumin (BSA) in PBS for 5 min and then permeabilized with $1 \%$ Triton X-100 for 25 min at RT. Sections were incubated with EdU reaction buffer (prepared in accordance to manufacturer's instructions) and washed 5 times in PBS for 3 min. Blocking was done by incubating the tissue with $4 \%$ fetal bovine serum (FBS) in $1 \%$ BSA in PBS for $1 \mathrm{~h}$ at RT, prior to incubation with primary antibodies (pericentriolar material 1 (PCM-1), at $1.2 \mu \mathrm{g} / \mathrm{ml}$ and Collagen IV (COLL IV) at $4 \mu \mathrm{g} / \mathrm{ml}$ in blocking solution) overnight, at $4^{\circ} \mathrm{C}$ (with gentle agitation). After washing three times in $0.1 \%$ Triton X-100 for 10 min (with agitation), sections were incubated with secondary antibodies (donkey anti-rabbit 488 at $4 \mu \mathrm{g} / \mathrm{ml}$ and donkey anti goat 568 at $4 \mu \mathrm{g} / \mathrm{ml}$ in blocking solution) for $3 \mathrm{~h}$ at RT (with gentle agitation) and again washed 3 times in $0.1 \%$ Triton X-100 in PBS (with agitation). Nuclei were counterstained by incubating sections 4',6'-diamino-2-fenil-indol (DAPI) $(10 \mu \mathrm{g} / \mathrm{ml})$ for $1 \mathrm{~h}$ at RT, respectively. Finally, following 3 washes in $0.1 \%$ Triton X-100 in PBS for $10 \mathrm{~min}$ (with agitation) sections were mounted in fluoroshield mounting media without DAPI and slides sealed using nail polish.

Notes:

(1) Recommended incubation volume: $250 \mu$ per section.

(2) To prevent the formation of spherical aberrations, which can introduce bias on the stereological analysis, the mounting medium should be chosen in accordance with the immersion liquid of the objective (i.e. both fluids should have similar refractive index).

Alternative version:

Wheat-germ agglutinin (WGA) can be used instead of COLL IV to stain cell boundaries. In this case, we recommend that WGA is incubated during the nuclei counterstaining with DAPI.

\section{Imaging}

Images of LV fragments were produced in a Leica TCS SP5 laser scanning confocal microscope. Images were acquired with a 40x/1.10 numerical aperture (NA) water immersion objective, using a $1 x$ optical zoom and bit depth set at 16 . Images of $1024 \times 1024$ pixels were obtained.

Emission was detected with hybrid and photomultiplier tube (PMT) detectors and gain and off-set values were defined to prevent saturated and empty pixels.

PCM-1 signal was used to define the beginning and ending of Z-Stack, which varied depending on the penetrance of the PCM-1 antibody. Images were composed of an average number of 40-50 stacks, corresponding to a thickness of 20-25 $\mu \mathrm{m}$. An average of ten images, from different fragments, were collected per heart.

After image acquisition, images were quantified with assistance of FIJI and IMARIS. Images for publication were deconvolved using Huygens Professional software. 


\section{Quantification of EdU ${ }^{+}$Cardiomyocytes and Binucleation}

With assistance of FIJl's cell counter tool, binucleation and the number of cardiomyocyte nuclei $\left(\mathrm{PCM}-1^{+}\right)$that incorporated $\mathrm{EdU}\left(\mathrm{EdU}^{+}\right)$were quantified. $\mathrm{PCM}-1^{+} \mathrm{EdU} \mathrm{U}^{+}$cells were considered when there was an overlap between PCM-1 (green), EdU (red) and DAPI (blue) (Figure $7 \mathrm{~A}$ - arrow). Cardiomyocytes $\left(\mathrm{PCM}-1^{+}\right)$were considered mononucleated when only one PCM-1 $1^{+}$nucleus was found within the cell, as delineated by WGA staining (Figure 7B magenta selection) and binucleated, when 2 or more nuclei were found (Figure 7B - orange selection). Cardiomyocyte density and binucleation were determined for each heart by averaging the result obtained in the different fragments. Hearts belonging to the same condition (i.e. sham or injury) were further grouped to determine the average cardiomyocyte density and binucleation.

Finally, the average number of nuclei per cardiomyocyte was calculated using the formula below:

\section{Average CM nuclei number $=2 \times(\%$ binucleation $)+1 \times(\%$ mononucleation $)$}

Note: nucleation was preferably evaluated in 3D sections containing longitudinally cut cardiomyocytes. See also supplemental movie 1.

A

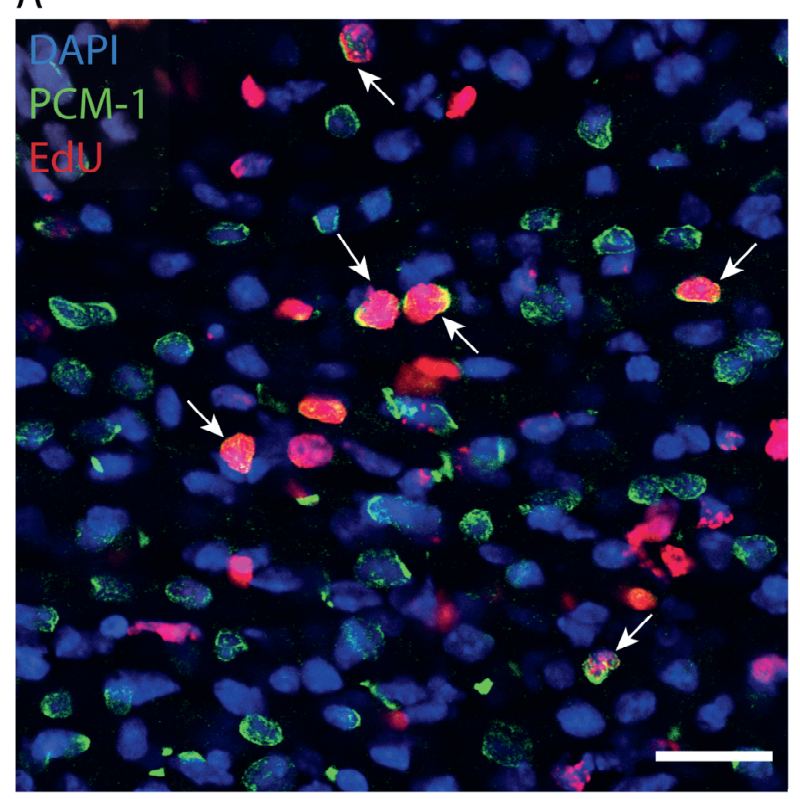

B

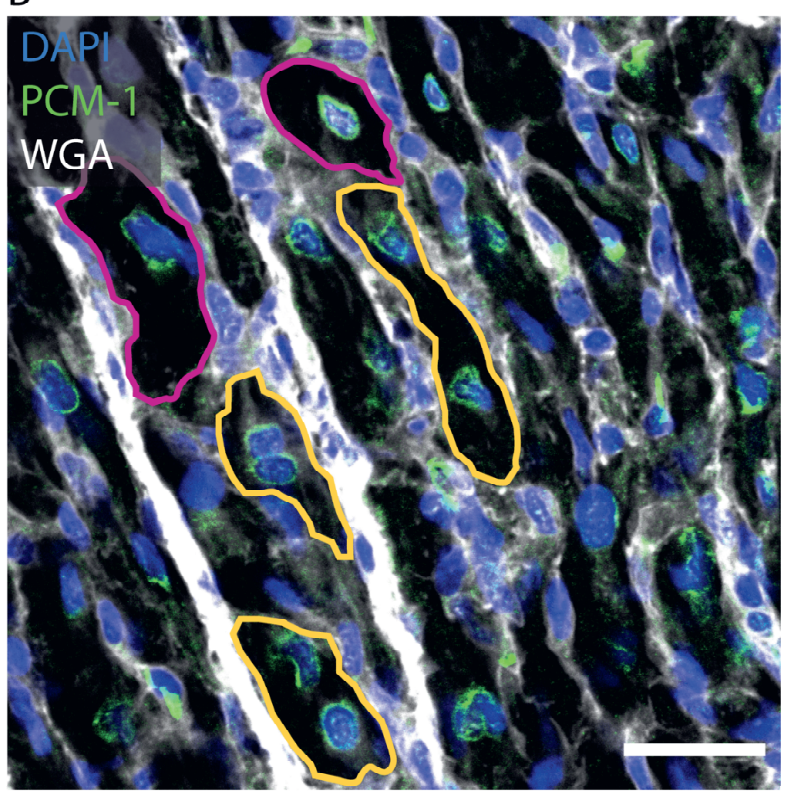

Figure 7 - A - Representative confocal image of a heart fragment stained for DAPI, PCM-1 and EdU. Arrows highlight cardiomyocyte nuclei that incorporated EdU (DAPI+PCM-1+EdU+). B - Representative confocal image of a heart fragment stained for DAPI, PCM-1 and WGA showing the methodology employed to determine cardiomyocyte nucleation. Cardiomyocytes were considered mononucleated or binucleated when 1 or 2 or more nuclei (DAPI+PCM-1+) were contained within the cell boundaries shown by WGA signal (mask in magenta or in orange, respectively). Scale bars: $25 \mu \mathrm{m}$. 


\section{Estimation of Total Number of Cardiomyocytes in the Left Ventricle}

The IMARIS's surface module assisted on determination of the total number of PCM-1 ${ }^{+}$ nuclei in a specific volume (image volume). This module was used to create semi-automatic segmentations of PCM-1 $1^{+}$nuclei and subsequent quantification, as well as to perform a 3D reconstruction of the image and determine its volume (Figure 8). PCM-1 signal was used to create the segmentation of cardiomyocyte nuclei, whereas remnant cardiac autofluorescence in the green channel was used to reconstruct the volume of each section. Smooth (surfaces detail) and split touching objects should be applied for PCM-1 ${ }^{+}$nuclei segmentation and should be adjusted to each specific dataset, maximizing the accuracy of the segmentation, yet maintained constant throughout the experiment. Threshold was set to avoid loss of information (false negatives) and the appearance of false positives.

After determining the total number of $\mathrm{PCM}-1^{+}$nuclei per image (with a specific volume), the cardiomyocyte nuclei density was determined dividing the number of $\mathrm{PCM}-1^{+}$per image by the image volume. As cardiomyocytes undergo binucleation, when determining the number of cardiomyocytes within an image, the cardiomyocyte nuclei density was divided by the average nuclei number per cardiomyocyte. Subsequently, the total number of cardiomyocytes in the LV is obtained by multiplying this value by the volume of the LV, as determined by histology, and considering a shrinking factor of $11.8 \%$ as follows:

$$
\text { CM number }=\frac{C M \text { nuclei density }}{\text { Average CM nuclei number }} \times \frac{\text { LV volume }}{0.882}
$$

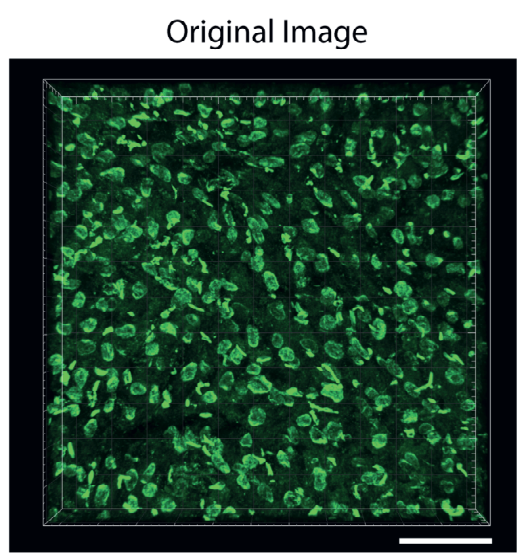

PCM-1+ nuclei segmentation
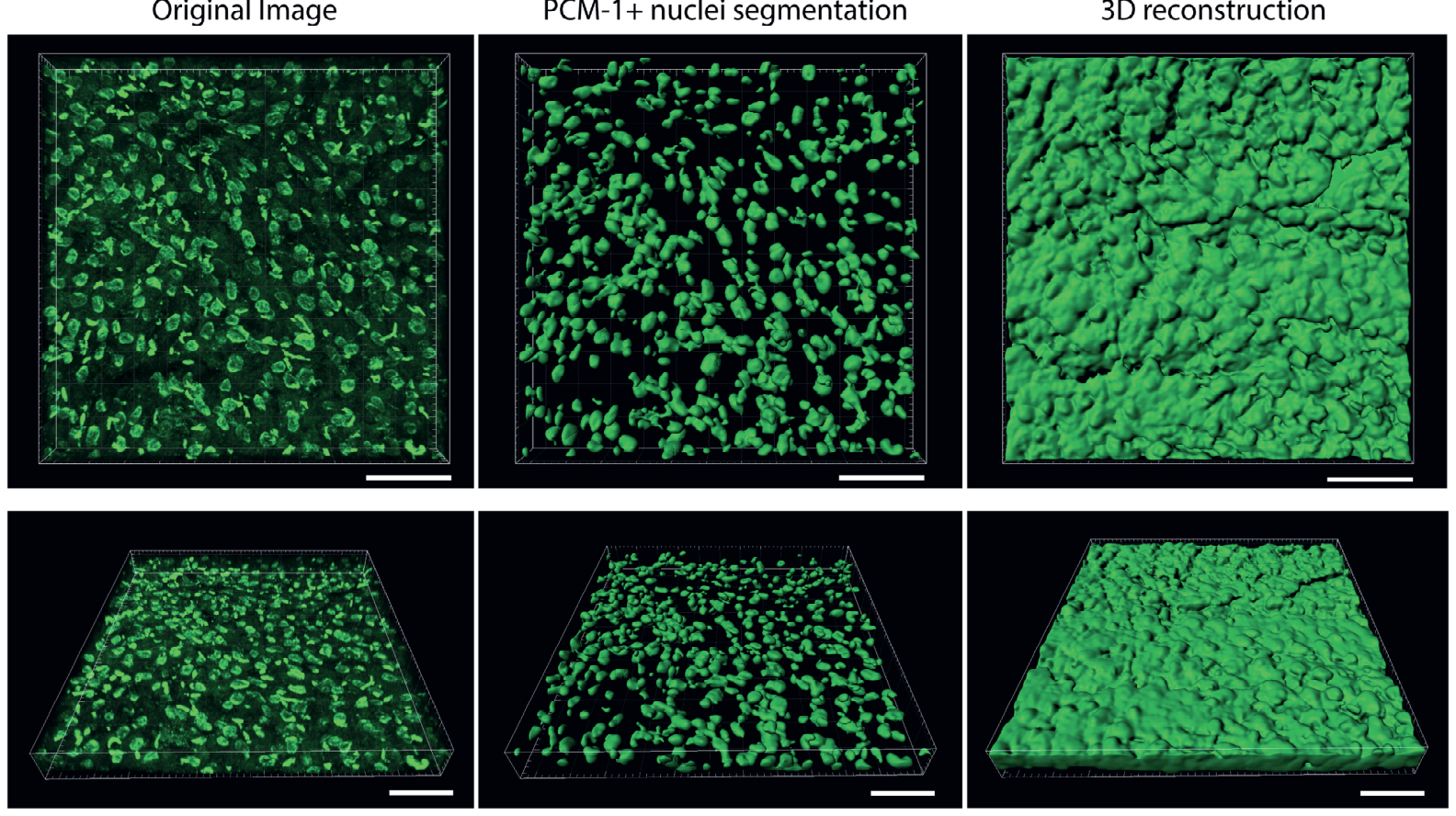

(legend on next page) 
Figure 8 - IMARIS semi-automatic segmentation of cardiomyocyte nuclei and image volume of a 40 $\mu \mathrm{m}$ section of a heart fragment. PCM-1 staining was used to segment cardiomyocyte nuclei while green autofluorescence was used to segment the image volume. From left to right different perspectives of a representative original image, segmentation of cardiomyocyte nuclei and 3D reconstruction of the $40 \mu \mathrm{m}$ section are displayed. Scale bars: $50 \mu \mathrm{m}$.

\section{Results and Discussion}

Proliferation of cardiomyocytes in adults is an extremely rare event. In fact, estimates suggest that less than $50 \%$ of cardiomyocytes are replaced during an average human life span [24]. Current line of thought advocates that low cardiomyocyte turnover is associated with the high specialization of this cell type. Indeed, during the postnatal period, cardiomyocytes undergo a complex maturation process characterized by transcriptomic, proteomic, metabolomic, morphologic and mechanic changes (reviewed in [25]). Adding further complexity to this, while in the maturation process, cardiomyocytes do not "simply" exit from the cell cycle as they continue to express some cell cycle markers and are responsive to external mitogenic stimuli [18]. As such, determination of cardiomyocyte proliferation, and especially at this period, can be deceiving because cardiomyocyte binucleation can be misinterpreted as cardiomyocyte proliferation.

Herein, we detail a protocol to determine cardiomyocyte proliferation and/or cardiomyocyte number in the heart. Using this protocol, we were able to solve a major question in the cardiovascular field: do neonatal murine hearts regenerate after apical resection? When P1 mice were subjected to apical resection we observed the activation of regenerative mechanisms, such as neovascularization and neomyogenesis. Stereological estimation of cardiomyocyte numbers revealed that the observed increase in cell cycle activity resulted from increased rates of cardiomyocyte proliferation, rather than binucleation. Even though we observed permanent fibrotic tissue deposition in the apex, the de novo generation of cardiomyocytes, more pronounced in regions proximal to the apex, compensated the myocardial loss and rendered normal systolic function to injured hearts [11].

This protocol is based on the study by Alkass and colleagues [26], whose aim was to evaluate if there was a cardiomyocyte number expansion in the preadolescent murine heart. Similarly, this protocol is based on the determination of cardiomyocyte nuclear density and myocardial volume. In contrast to this study, we determined the myocardial volume by quantifying the LV area, using representative sequential sections of paraffin-embedded hearts, which was multiplied by the distance separating adjacent sections to obtain an accurate estimation of the LV volume. This differs from previous studies in which the volume of the heart was calculated by a transformation of the wet weight of the formalinfixed LV into volume using the ratio $1.06 \mathrm{~g} / \mathrm{cm}^{3}$. To our understanding, our protocol allows a far more reliable estimation of the myocardial volume as mass variations in the dissection of the LV and liquid content in the ventricle may undermine accurate estimations. 
This alternative protocol has, however, the limitation that FFPE tissues tend to shrink. Yet, by determining the ratio between fresh and processed dimensions of the heart we were able to correct for this shrinkage.

By combining the power of 3D-imaging with stereology, our protocol surpasses the limitations of traditional methods used to estimate cardiomyocyte proliferation ex vivo (e.g. immunodetection of cell cycle markers and/or incorporation of thymidine analogs). Resorting to $40 \mu \mathrm{m}$ thick sections, this protocol allows for the examination of several layers of cells within the same section. As such, we were able to simultaneously evaluate the incorporation of EdU by cardiomyocytes and measure their nucleation within the myocardium.

Owing to the biomedical relevance of understanding the mechanisms governing cardiomyocyte proliferation, other methodologies have been proposed and applied. In 2006, the patterning of Anillin and Aurora B was proposed as a predictor of cardiomyocyte binucleation/proliferation [27]. During anaphase, anillin is localized in the cell cortex, where it crosslinks cytoskeletal components, whereas aurora B is located in the midzone and is responsible for the location of centralspindlin to the central spindle. Both proteins are required for the successful progression of cytokinesis and for this reason they are often considered as a better readout of late cell division. In binucleating cells, anillin does not have a cortical localization thus leading to an asymmetric furrow ingression which precludes efficient cell division. Hence, by studying the localization of anillin in cell cycle active cardiomyocytes we may anticipate whether that activity is leading to true cardiomyocyte proliferation or to binucleation.

More recently, the position of the midbody and the distance between daughter nuclei were also proposed as bona fide markers of cardiomyocyte proliferation or binucleation [28]. According to Hesse and colleagues, Anillin expression per se is not sufficient to conclude whether a cell divides or binucleates but, instead, its location is much more informative. While irregular midbodies anticipate binucleation, regular midbodies correlate with effective cell division. Moreover, the distance between daughter nuclei was found to be shorter in irregular/binucleating cardiomyocytes when compared to regular/dividing cardiomyocytes.

Regardless of the accuracy of each of these methods to discriminate cardiomyocyte proliferation from binucleation, both strategies have limited application for in vivo studies as both depend on capturing very scarce events. In addition, tissue complexity often hinders such detailed analysis of subcellular processes (e.g. identification of regular/irregular midbody formation). Stereology, as a tool to assess the number of cardiomyocytes, is more reliable because it does not depend on seizing the exact moment in which cells divide. It rather evaluates the outcome of such division by assessing the number of cells present at a later point in time. Of importance, herein, we detail a protocol combining stereology with an EdU pulse-chase experiment allowing further estimation of newly generated cardiomyocytes at a given period.

Aiming to differentiate cardiomyocyte proliferation from binucleation, Ali et al. proposed the mosaic analysis with double markers (MADM) [29]. In this system, the two daughter 
cells of a dividing cell will be uniquely labeled with red fluorescent protein (RFP) or green fluorescent protein (GFP) and if binucleation takes place, both fluorescent proteins will be co-expressed and yellow signal is detected. This seems to be a robust tool to assess cardiomyocyte proliferation and/or binucleation but similarly to other lineage tracing methods has the caveat of using multiple reporters even before other alleles of interest are incorporated. Although more laborious, stereology presents itself as a valid and more reliable alternative that can be used in combination with genetic systems.

Not depending on genetic manipulation nor on spatial-temporal detection of $\mathrm{CM}$ proliferation/binucleation, cardiomyocyte number can be assessed employing enzymatic digestion and counting using, for example, a flow cytometer [30]. Yet, cardiomyocytes, especially adult, are very sensitive to enzymatic degradation and require laborious and specific equipment for efficient isolation [31, 32]. Larger cardiomyocytes also impose technical difficulties for flow cytometry (FC) as these cells can easily break and/or interrupt the fluidics. Hence, by using this approach, a large percentage of cardiomyocyte are lost during the isolation process leading to the underestimation of cardiomyocyte numbers. More worryingly, a selection bias will be created by focusing the analysis on smaller/immature cardiomyocytes that show better survival throughout the isolation procedure and are more compatible with fluorescence activated cell sorting (FACS) fluidics. In spite of recent technological and methodological advances, a simple and reproducible tool to assess cardiomyocyte proliferation/number was still lacking. Our protocol will be certainly useful for those willing to evaluate the regenerative capacity of the heart. Alike all referred methodologies, stereology fails to detect cardiomyocytes that underwent endoreplication of DNA and become polyploid. Assessment of nuclear ploidy is typically performed by a complementary technique involving cardiomyocyte nuclei isolation and characterization by FC [26]. When conducted, however, the information about the number of nuclei per cardiomyocyte is lost.

In our understanding, despite being a labor-intensive technique, stereology faithfully estimates the number of cardiomyocytes. This optimized protocol is of broad interest and direct relevance to researchers aiming to determine cardiomyocyte proliferation and/or number in vivo, namely to study molecular mechanisms regulating cardiomyocyte proliferation, both at a steady state or in response to different types of stimuli.

\section{Acknowledgements}

This work was funded by the European Regional Development Fund (ERDF) through COMPETE 2020, Portugal 2020 and by FCT (Fundação para a Ciência e Tecnologia, [POCI-010145-FEDER-030985]); and by FCT/Ministério da Ciência, Tecnologia e Inovação in the framework of individual funding: [SFRH/BD/111799/2015] to V.S.-P., [PD/BD/127997/2016] to T.L.L., [SFRH/BD/144769/2019] to E.S. and [CEECINST/00091/2018] to DSN. The authors acknowledge the support of i3S Scientific Platform Advanced Light Microscopy, member of the national infrastructure PPBI-Portuguese Platform of Biolmaging (supported by POCI-01- 


\section{5-FEDER-022122). PDCM was supported by a Dutch Heart Foundation grant (NHS2015T066).}

\section{References}

[1] O. Bergmann, S. Zdunek, A. Felker, M. Salehpour, K. Alkass, S. Bernard, S.L. Sjostrom, M. Szewczykowska, T. Jackowska, C. Dos Remedios, T. Malm, M. Andra, R. Jashari, J.R. Nyengaard, G. Possnert, S. Jovinge, H. Druid, J. Frisen, Dynamics of Cell Generation and Turnover in the Human Heart, Cell 161(7) (2015) 1566-75.

[2] D.F. Goldspink, J.G. Burniston, L.B. Tan, Cardiomyocyte death and the ageing and failing heart, Experimental physiology 88(3) (2003) 447-458.

[3] E. Cambria, F.S. Pasqualini, P. Wolint, J. Gunter, J. Steiger, A. Bopp, S.P. Hoerstrup, M.Y. Emmert, Translational cardiac stem cell therapy: advancing from first-generation to next-generation cell types, NPJ Regenerative medicine 2(1) (2017) 17.

[4] A.C. Silva, S.C. Rodrigues, J. Caldeira, A.M. Nunes, V. Sampaio-Pinto, T.P. Resende, M.J. Oliveira, M.A. Barbosa, S. Thorsteinsdottir, D.S. Nascimento, O.P. Pinto-do, Three-dimensional scaffolds of fetal decellularized hearts exhibit enhanced potential to support cardiac cells in comparison to the adult, Biomaterials 104 (2016) 52-64.

[5] Y. Song, C. Zhang, J. Zhang, N. Sun, K. Huang, H. Li, Z. Wang, K. Huang, L. Wang, An injectable silk sericin hydrogel promotes cardiac functional recovery after ischemic myocardial infarction, Acta biomaterialia 41 (2016) 210-23.

[6] Y.W. Liu, B. Chen, X. Yang, J.A. Fugate, F.A. Kalucki, A. Futakuchi-Tsuchida, L. Couture, K.W. Vogel, C.A. Astley, A. Baldessari, J. Ogle, C.W. Don, Z.L. Steinberg, S.P. Seslar, S.A. Tuck, H. Tsuchida, A.V. Naumova, S.K. Dupras, M.S. Lyu, J. Lee, D.W. Hailey, H. Reinecke, L. Pabon, B.H. Fryer, W.R. MacLellan, R.S. Thies, C.E. Murry, Human embryonic stem cell-derived cardiomyocytes restore function in infarcted hearts of non-human primates, Nature biotechnology 36(7) (2018) 597-605.

[7] Y. Shiba, T. Gomibuchi, T. Seto, Y. Wada, H. Ichimura, Y. Tanaka, T. Ogasawara, K. Okada, N. Shiba, K. Sakamoto, D. Ido, T. Shiina, M. Ohkura, J. Nakai, N. Uno, Y. Kazuki, M. Oshimura, I. Minami, U. Ikeda, Allogeneic transplantation of iPS cell-derived cardiomyocytes regenerates primate hearts, Nature 538(7625) (2016) 388-391. [8] K. Gabisonia, G. Prosdocimo, G.D. Aquaro, L. Carlucci, L. Zentilin, I. Secco, H. Ali, L. Braga, N. Gorgodze, F. Bernini, S. Burchielli, C. Collesi, L. Zandona, G. Sinagra, M. Piacenti, S. Zacchigna, R. Bussani, F.A. Recchia, M. Giacca, MicroRNA therapy stimulates uncontrolled cardiac repair after myocardial infarction in pigs, Nature 569(7756) (2019) 418-422.

[9] T.M.A. Mohamed, Y.S. Ang, E. Radzinsky, P. Zhou, Y. Huang, A. Elfenbein, A. Foley, S. Magnitsky, D. Srivastava, Regulation of Cell Cycle to Stimulate Adult Cardiomyocyte Proliferation and Cardiac Regeneration, Cell 173(1) (2018) 104-116 e12.

[10] E.R. Porrello, A.I. Mahmoud, E. Simpson, J.A. Hill, J.A. Richardson, E.N. Olson, H.A. Sadek, Transient regenerative potential of the neonatal mouse heart, Science (New York, N.Y.) 331(6020) (2011) 1078-80.

[11] V. Sampaio-Pinto, S.C. Rodrigues, T.L. Laundos, E.D. Silva, F. Vasques-Novoa, A.C. Silva, R.J. Cerqueira, T.P. Resende, N. Pianca, A. Leite-Moreira, G. D'Uva, S. Thorsteinsdottir, O.P. Pinto-do, D.S. Nascimento, Neonatal Apex Resection Triggers Cardiomyocyte Proliferation, Neovascularization and Functional Recovery Despite Local Fibrosis, Stem cell reports 10(3) (2018) 860-874.

[12] K. Kretzschmar, Y. Post, M. Bannier-Helaouet, A. Mattiotti, J. Drost, O. Basak, V.S.W. Li, M. van den Born, Q.D. Gunst, D. Versteeg, L. Kooijman, S. van der Elst, J.H. van Es, E. van Rooij, M.J.B. van den Hoff, H. Clevers, Profiling proliferative cells and their progeny in damaged murine hearts, Proceedings of the National Academy of Sciences of the United States of America 115(52) (2018) E12245-E12254.

[13] Y. Zhang, N. Gago-Lopez, N. Li, Z. Zhang, N. Alver, Y. Liu, A.M. Martinson, A. Mehri, W.R. MacLellan, Single-cell imaging and transcriptomic analyses of endogenous cardiomyocyte dedifferentiation and cycling, Cell discovery 5 (2019) 30.

[14] J. Piquereau, R. Ventura-Clapier, Maturation of Cardiac Energy Metabolism During Perinatal Development, Frontiers in physiology 9 (2018) 959.

[15] M.A. Laflamme, C.E. Murry, Heart regeneration, Nature 473(7347) (2011) 326-35.

[16] A.N. Paradis, M.S. Gay, L. Zhang, Binucleation of cardiomyocytes: the transition from a proliferative to a terminally differentiated state, Drug Discov Today 19(5) (2014) 602-9. 
[17] M.H. Soonpaa, K.K. Kim, L. Pajak, M. Franklin, L.J. Field, Cardiomyocyte DNA synthesis and binucleation during murine development, The American journal of physiology 271(5 Pt 2) (1996) H2183-9.

[18] D.C. Zebrowski, R. Becker, F.B. Engel, Towards regenerating the mammalian heart: challenges in evaluating experimentally induced adult mammalian cardiomyocyte proliferation, American journal of physiology. Heart and circulatory physiology 310(9) (2016) H1045-54.

[19] J. Schindelin, I. Arganda-Carreras, E. Frise, V. Kaynig, M. Longair, T. Pietzsch, S. Preibisch, C. Rueden, S. Saalfeld, B. Schmid, J.Y. Tinevez, D.J. White, V. Hartenstein, K. Eliceiri, P. Tomancak, A. Cardona, Fiji: an open-source platform for biological-image analysis, Nat Methods 9(7) (2012) 676-82.

[20] D. Sage, L. Donati, F. Soulez, D. Fortun, G. Schmit, A. Seitz, R. Guiet, C. Vonesch, M. Unser, DeconvolutionLab2: An open-source software for deconvolution microscopy, Methods (San Diego, Calif.) 115 (2017) 28-41.

[21] C. Zeng, F. Pan, L.A. Jones, M.M. Lim, E.A. Griffin, Y.I. Sheline, M.A. Mintun, D.M. Holtzman, R.H. Mach, Evaluation of 5-ethynyl-2'-deoxyuridine staining as a sensitive and reliable method for studying cell proliferation in the adult nervous system, Brain Res 1319 (2010) 21-32.

[22] P.B. Dobrin, Effect of histologic preparation on the cross-sectional area of arterial rings, The Journal of surgical research 61(2) (1996) 413-5.

[23] H. Lum, W. Mitzner, Effects of $10 \%$ formalin fixation on fixed lung volume and lung tissue shrinkage. A comparison of eleven laboratory species, The American review of respiratory disease 132(5) (1985) 1078-83.

[24] O. Bergmann, R.D. Bhardwaj, S. Bernard, S. Zdunek, F. Barnabe-Heider, S. Walsh, J. Zupicich, K. Alkass, B.A. Buchholz, H. Druid, S. Jovinge, J. Frisen, Evidence for cardiomyocyte renewal in humans, Science (New York, N.Y.) 324(5923) (2009) 98-102.

[25] G.J. Scuderi, J. Butcher, Naturally Engineered Maturation of Cardiomyocytes, Front Cell Dev Biol 5(50) (2017) 50.

[26] K. Alkass, J. Panula, M. Westman, T.D. Wu, J.L. Guerquin-Kern, O. Bergmann, No Evidence for Cardiomyocyte Number Expansion in Preadolescent Mice, Cell 163(4) (2015) 1026-36.

[27] F.B. Engel, M. Schebesta, M.T. Keating, Anillin localization defect in cardiomyocyte binucleation, Journal of molecular and cellular cardiology 41(4) (2006) 601-12.

[28] M. Hesse, M. Doengi, A. Becker, K. Kimura, N. Voeltz, V. Stein, B.K. Fleischmann, Midbody Positioning and Distance Between Daughter Nuclei Enable Unequivocal Identification of Cardiomyocyte Cell Division in Mice, Circulation research 123(9) (2018) 1039-1052.

[29] S.R. Ali, S. Hippenmeyer, L.V. Saadat, L. Luo, I.L. Weissman, R. Ardehali, Existing cardiomyocytes generate cardiomyocytes at a low rate after birth in mice, Proceedings of the National Academy of Sciences of the United States of America 111(24) (2014) 8850-5.

[30] F.B. Engel, M. Schebesta, M.T. Duong, G. Lu, S. Ren, J.B. Madwed, H. Jiang, Y. Wang, M.T. Keating, p38 MAP kinase inhibition enables proliferation of adult mammalian cardiomyocytes, Genes \& development 19(10) (2005) 1175-87.

[31] M.N. Berry, D.S. Friend, J. Scheuer, Morphology and metabolism of intact muscle cells isolated from adult rat heart, Circulation research 26(6) (1970) 679-87.

[32] T. Kono, Roles of collagenases and other proteolytic enzymes in the dispersal of animal tissues, Biochimica et biophysica acta 178(2) (1969) 397-400. 


\section{A stereological perspective on cardiac ontology}

Vasco Sampaio-Pinto ${ }^{a, b, c, d, e}$, Andrea Raso ${ }^{d, e}$, Elsa D Silva ${ }^{a, b}$, Tiago L Laundos ${ }^{a, b, c}$, Perpétua Pinto-do-Óa,b,c, Paula da Costa Martins ${ }^{\mathrm{d}, \mathrm{e}, \mathrm{f}}$, Leon J de Windt ${ }^{\mathrm{d}, \mathrm{e}}$, Diana S Nascimento ${ }^{\mathrm{a}, \mathrm{b}, \mathrm{c}}$

a i3S - Instituto de Investigação e Inovação em Saúde, Universidade do Porto, Porto, Portugal;

b INEB - Instituto Nacional de Engenharia Biomédica, Universidade do Porto, Porto, Portugal;

c ICBAS - Instituto de Ciências Biomédicas Abel Salazar, Universidade do Porto, Portugal;

${ }^{d}$ Department of Cardiology, CARIM School for Cardiovascular Diseases, Faculty of Health, Medicine and Life Sciences, Maastricht University, Maastricht, The Netherlands;

e Department of Molecular Genetics, Faculty of Sciences and Engineering, Maastricht University, Maastricht, The Netherlands;

${ }^{f}$ Department of Physiology and Cardiothoracic Surgery, Faculty of Medicine, University of Porto, Porto, Portugal.

in preparation 


\section{Introduction}

The view of the mammalian heart as a post-mitotic organ was recently challenged by accumulating evidence of postnatal cardiomyocyte proliferation. Taking advantage of carbon-14 integration during DNA replication, Bergman and colleagues demonstrated that cardiomyocytes renew in the adult human myocardium [1]. Even though this capacity was shown to be potentiated in response to an injury [2] the low turnover rates were incompatible with efficient cardiac regeneration.

In order to gain mechanistic insight on the rate and origin of newly formed cardiomyocytes, different lineage-tracing and injury models were used and unveiled that newly formed cardiomyocytes arise from the proliferation of pre-existing ones, rather than by differentiation of cardiac-resident progenitor cells [3, 4].

Yet, in contrast to lower vertebrates that exhibit lifelong cardiac regeneration via proliferation of pre-existing cardiomyocytes [5-7], mammalian cardiomyocyte renewal is scarce and inversely related with aging $[3,8]$. In 2011, pioneer work by Porrello et al. showed that this regenerative capacity was also present in murine hearts but limited to the first days of life [9]. Subsequent work identified similarities between the hearts of adult zebrafish and neonatal mice, explaining in part the development of a regenerative response following cardiac damage. Both fetal murine and zebrafish hearts obtain energy via glycolysis $[10,11]$ but shortly after birth, murine cardiomyocytes face increasing oxygen levels, which leads to a metabolic shift towards oxidative phosphorylation of fatty acids [10, 12]. This oxidative environment is responsible for the formation of reactive oxygen species and activation of DNA damage response, withdrawing cardiomyocytes from the cell cycle [10]. Furthermore, cardiomyocytes in murine hearts undergo maturation shortly after birth and become binucleated as the result of incomplete cytokinesis [13]. This added genetic material is considered to negatively impact on the capacity of cardiomyocytes to undergo mitosis and cell division $[14,15]$.

Hence, it is hypothesized that during the neonatal period hearts grow via a combination of hyperplasia, as a minor subset of cardiomyocytes preserve the embryonic phenotype and proliferate, and hypertrophy where cardiomyocytes grow in size by the longitudinal addition of sarcomeres. This hypertrophic growth at the cellular level translates into an increased contraction force of the heart, needed to sustain the increasing metabolic demands of a growing body plan [16].

Although the transition from cardiomyocyte hyperplasia to hypertrophy occurring around birth is well documented, a complete understanding of the cellular and extracellular elements governing cardiac regeneration is still lacking. Furthermore, as previously discussed, the absence of systematic and reliable techniques to assess cardiomyocyte proliferation hampers progress in the field.

Making use of the methodology reported in the preceding subchapter, we document the hyperplasic response of neonatal hearts at two different timepoints (i.e. P6 and P12). In agreement with the literature we observe that hearts grow mainly by hypertrophy from P6 to $\mathrm{P} 12$, accompanied by increasing rates of binucleation and reduced cardiomyocyte 
nuclear density. Nonetheless, stereological estimation revealed that P12 hearts have more cardiomyocytes than the P6 counterparts, suggesting that cardiomyocyte proliferation still occurs and partially contributes to heart growth.

\section{Methods}

In order to label replicating cells, EdU was intraperitoneally injected at P1, P5 and P9 at a concentration of $25 \mu \mathrm{g} / \mathrm{g}$ of body weight. Half of the animals were euthanized at P6 while the remaining were euthanized at P12 (Figure 9). Once harvested, the hearts were processed for myocardial volume and cardiomyocyte density assessment as described.

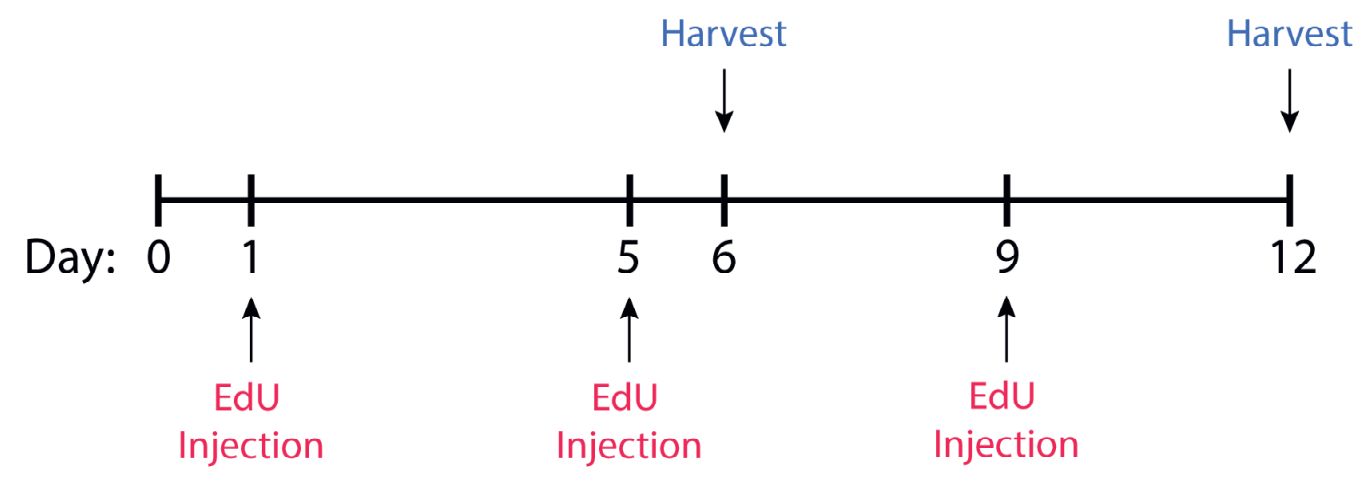

Figure 9 - Timeline of procedures involving animals. EdU was subcutaneously injected at P1 and P5 and a fraction of animals were euthanized at P6. The remaining received an additional EdU injection at P9 and were euthanized at P12.

\section{Results}

The postnatal period is characterized by extensive body growth. Estimation of the left ventricle volume revealed a substantial increase from P6 to P12, paralleled by a rise in body weight (Figure 10A).

From P6 to P12, the number of cardiomyocyte nuclei that had incorporated EdU (PCM$1^{+} \mathrm{EdU}^{+}$) per $\mathrm{mm}^{3}$ was significantly reduced (Figure 10B). Similarly, the cardiomyocyte nucleus density (i.e. number of cardiomyocytes per $\mathrm{mm}^{3}$ ) was reduced in P12 hearts when compared to the younger P6 (Figure 10C). Such observations are explained by the hypertrophic growth of cardiomyocytes, expected at this ontogenic timepoint (Figure 10D). As a consequence, cardiomyocytes become less dense in the myocardium (i.e. lower cardiomyocyte number per $\mathrm{mm}^{3}$ of myocardium). This is particularly noticeable in the representative images of the myocardium of P6 and P12 hearts (Figure 10G), where the number of $\mathrm{PCM}-1^{+}$and $\mathrm{PCM}-1^{+} \mathrm{EdU} \mathrm{U}^{+}$nuclei in the same area is appreciable decreased at P12 compared to P6. Consistently, the percentage of binucleated cardiomyocytes was significantly increased in P12 versus P6 hearts, confirming that maturation of cardiomyocytes took place during this period (Figure 10E). 
Finally, assuming the cardiomyocyte nucleus density and the left ventricle volume determined previously, the total number of cardiomyocytes in the left ventricle was determined and found to be significantly higher in P12 hearts compared to P6 (Figure 10F). Hence, despite maturation and hypertrophic growth of cardiomyocytes are the major players in cardiac development from P6 to P12, hyperplasia contributes minimally yet significantly to the emergence of hearts with more cardiomyocytes.

A
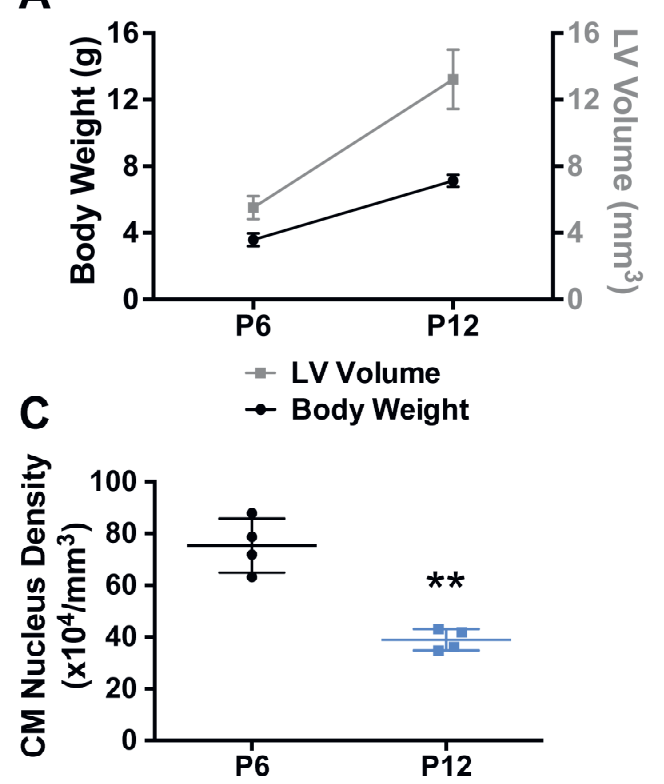

$E$

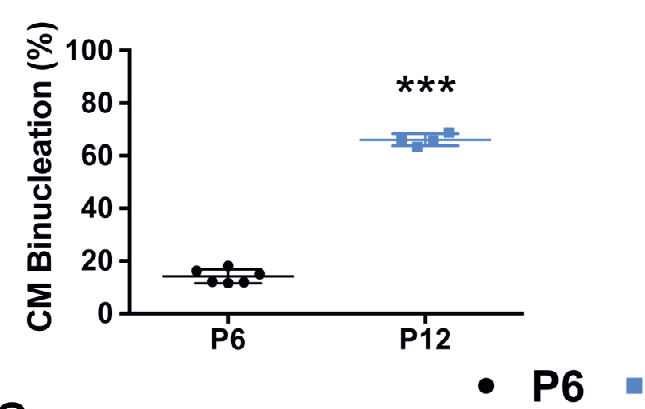

B

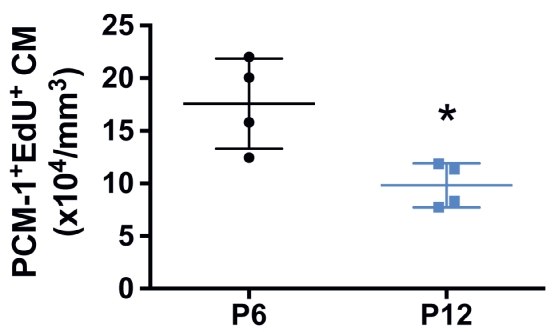

D

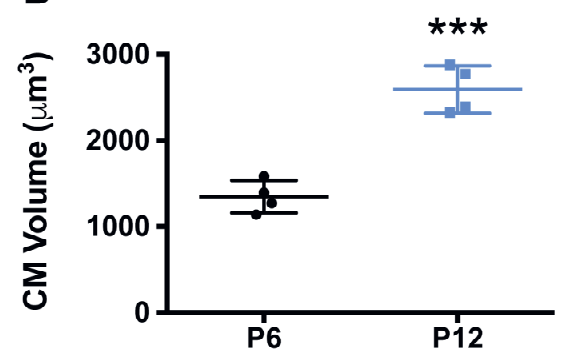

$\mathbf{F}$

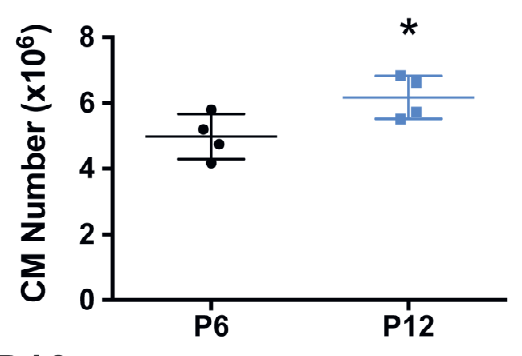

P12

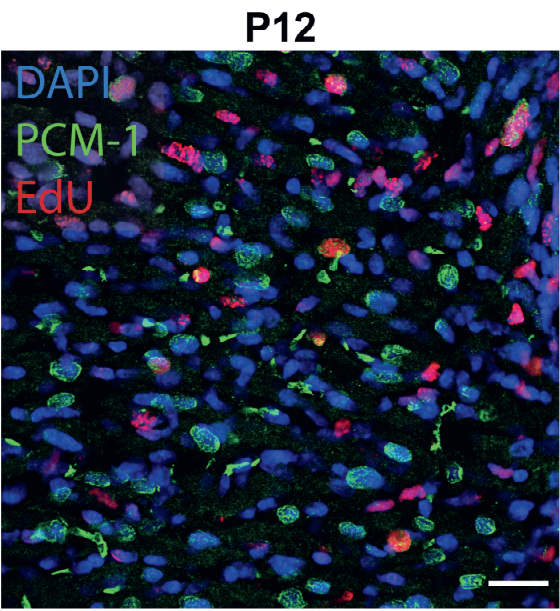

(legend on next page) 
Figure 10 - A - Estimation of left ventricle volume of P6 and P12 hearts. B - Quantification of EdU incorporation in $\mathrm{PCM}-1^{+}$cardiomyocytes at P6 and P12. C - Cardiomyocyte nucleus density in the left ventricle of $P 6$ and $P 12$ hearts. $D$ - Cardiomyocyte volume in the left ventricle of $P 6$ and $P 12$ hearts. $\mathrm{E}-$ Cardiomyocyte binucleation percentage in the left ventricle of P6 and P12 hearts. F-Stereological estimation of cardiomyocyte number in the left ventricle of P6 and P12 hearts. G - Representative confocal images of PCM-1+EdU+ cardiomyocytes in the left ventricle of P6 and P12 hearts. Scale-bars: $25 \mu \mathrm{m}$.

\section{Discussion}

Despite significant effort of clinicians and researchers to lower the social-economic burden of cardiovascular diseases, they still stand as the main cause of death worldwide [17]. The little regenerative potential of the mammalian heart explains, at least in part, the epidemiology of cardiovascular diseases. In fact, adult cardiomyocyte proliferation in human hearts was found to be very scarce both at steady state [1] and after injury [2].

Yet, promising studies revealed that neonatal mammals display, alike lower vertebrates [57], the ability of regenerating the myocardium via proliferation of pre-existing cardiomyocytes [9, 18-22]. Regrettably, this capacity diminishes rapidly with age in a process regulated by cell-intrinsic and extrinsic mechanisms (reviewed in [23]). While the majority of the research has been focusing on identifying mechanisms regulating cardiomyocyte proliferation and testing their therapeutic potential in a non-regenerative heart, studies systematically assessing the endogenous proliferative capacity of neonatal hearts are missing. Moreover, the field lacks standardized and reliable techniques to assess proliferation in vivo/ex vivo, which hinders comparisons across studies.

What is the exact contribution of hypertrophy and hyperplasia to the heart at a given point in time? Is the proliferation of cardiomyocytes in the neonatal heart a remnant of embryonic development or the result of secondary activations? Is it enough to boost cardiomyocyte proliferation to achieve cardiac regeneration? These are questions only partially addressed in the cardiovascular field that would benefit from the application of the methodology detailed in the herein thesis.

We subjected P6 and P12 murine hearts to stereological estimation of cardiomyocyte numbers. This technique revealed that $\mathrm{P} 12$ hearts are composed by more cardiomyocytes in comparison to P6 (i.e. 24\% increase), supporting that cardiomyocyte hyperplasia still takes place after P6. In parallel, we observed a profound remodeling of the left ventricle from P6 to P12, with the majority of cardiomyocytes becoming binucleated and hypertrophic, as confirmed increased cardiomyocyte volume and reduced cardiomyocyte nucleus density on P12 hearts. These findings are in line with a previous report by Alkass et al. where P12 hearts were estimated to have approximately $18 \%$ more cardiomyocytes than P6 hearts [8] and diverge from the work from Naqvi et al. that reported a milder increase of $\sim 8 \%$ during the same timeframe [24]. Noteworthy, Naqvi and colleagues report a secondary peak of cardiomyocyte proliferation caused by an upsurge in thyroid hormone in preadolescent mice [24], which failed to be replicated by Alkass et al. [8]. As opportunely 
discussed, adoption of different methodologies to determine cardiomyocyte numbers and/or proliferation might have been on the origin of the conflicting results $[25,26]$.

Notwithstanding, the work herein and above-mentioned publications support the existence of cardiomyocytes able to proliferate in non-regenerative murine hearts. Hence, the loss of cardiac regeneration during postnatal development does not seem to exclusively driven by reduced cardiomyocyte proliferation, but is likely affected by factors such as extracellular matrix stiffness [27], energy metabolism [28] and oxygen concentration [10].

The work in this subchapter reiterates the message conveyed previously that the field needs systematic studies exploring the proliferative capacity of cardiomyocytes at different timepoints and across mammalian species. Furthermore, as most techniques do not reliably distinguish cardiomyocyte proliferation from cardiomyocyte binucleation [29], stereology is a valid and reliable methodology to assess true cardiomyocyte hyperplasia.

The herein study proved that stereology is sufficiently robust to detect cardiomyocyte number differences between P6 and P12 hearts, despite the major influence of cardiomyocyte hypertrophy for cardiac growth between these timepoints. As such, this study can be further applied to older animals and to intermediate timepoints to fully elucidate on the proliferative potential of neonatal hearts. This is of pivotal importance since determining the temporal window in which neonatal hearts exhibit cardiomyocyte proliferation has profound impact on the way researchers look for neomyogenic cues to develop new therapeutic strategies to regenerate the adult heart.

\section{Acknowledgements}

This work was funded by the European Regional Development Fund (ERDF) through COMPETE 2020, Portugal 2020 and by FCT (Fundação para a Ciência e Tecnologia, [POCI-010145-FEDER-030985]); and by FCT/Ministério da Ciência, Tecnologia e Inovação in the framework of individual fellowships: SFRH/BD/111799/2015 to V.S.-P., PD/BD/127997/2016 to T.L.L. and SFRH/BD/144769/2019 to E.D.S.

L.D.W. acknowledges support from the Netherlands CardioVascular Research Initiative: the Dutch Heart Foundation, Dutch Federation of University Medical Centers, ZonMW and the Royal Netherlands Academy of Sciences (CVON2017-ARENA PRIME). L.D.W. was further supported by ERC Consolidator Grant 311549 CALMIRS and a VICl award 918-156-47 from NWO. PDCM was supported by a Dutch Heart Foundation grant (NHS2015T066).

\section{References}

[1] O. Bergmann, R.D. Bhardwaj, S. Bernard, S. Zdunek, F. Barnabe-Heider, S. Walsh, J. Zupicich, K. Alkass, B.A. Buchholz, H. Druid, S. Jovinge, J. Frisen, Evidence for cardiomyocyte renewal in humans, Science 324(5923) (2009) 98-102.

[2] A.P. Beltrami, K. Urbanek, J. Kajstura, S.M. Yan, N. Finato, R. Bussani, B. Nadal-Ginard, F. Silvestri, A. Leri, C.A. Beltrami, P. Anversa, Evidence that human cardiac myocytes divide after myocardial infarction, The New England journal of medicine 344(23) (2001) 1750-7.

[3] S.E. Senyo, M.L. Steinhauser, C.L. Pizzimenti, V.K. Yang, L. Cai, M. Wang, T.D. Wu, J.L. Guerquin-Kern, C.P. Lechene, R.T. Lee, Mammalian heart renewal by pre-existing cardiomyocytes, Nature 493(7432) (2013) 433-6. 
[4] J.H. van Berlo, O. Kanisicak, M. Maillet, R.J. Vagnozzi, J. Karch, S.C. Lin, R.C. Middleton, E. Marban, J.D. Molkentin, c-kit+ cells minimally contribute cardiomyocytes to the heart, Nature 509(7500) (2014) 337-41.

[5] I.L. Flink, Cell cycle reentry of ventricular and atrial cardiomyocytes and cells within the epicardium following amputation of the ventricular apex in the axolotl, Amblystoma mexicanum: confocal microscopic immunofluorescent image analysis of bromodeoxyuridine-labeled nuclei, Anatomy and embryology 205(3) (2002) 235-44.

[6] F. Laube, M. Heister, C. Scholz, T. Borchardt, T. Braun, Re-programming of newt cardiomyocytes is induced by tissue regeneration, Journal of cell science 119(22) (2006) 4719-4729.

[7] K.D. Poss, L.G. Wilson, M.T. Keating, Heart regeneration in zebrafish, Science 298(5601) (2002) 2188-90.

[8] K. Alkass, J. Panula, M. Westman, T.D. Wu, J.L. Guerquin-Kern, O. Bergmann, No Evidence for Cardiomyocyte Number Expansion in Preadolescent Mice, Cell 163(4) (2015) 1026-36.

[9] E.R. Porrello, A.I. Mahmoud, E. Simpson, J.A. Hill, J.A. Richardson, E.N. Olson, H.A. Sadek, Transient regenerative potential of the neonatal mouse heart, Science 331(6020) (2011) 1078-80.

[10] B.N. Puente, W. Kimura, S.A. Muralidhar, J. Moon, J.F. Amatruda, K.L. Phelps, D. Grinsfelder, B.A. Rothermel, R. Chen, J.A. Garcia, C.X. Santos, S. Thet, E. Mori, M.T. Kinter, P.M. Rindler, S. Zacchigna, S. Mukherjee, D.J. Chen, A.I. Mahmoud, M. Giacca, P.S. Rabinovitch, A. Aroumougame, A.M. Shah, L.I. Szweda, H.A. Sadek, The oxygen-rich postnatal environment induces cardiomyocyte cell-cycle arrest through DNA damage response, Cell 157(3) (2014) 565-79.

[11] V. Sander, G. Sune, C. Jopling, C. Morera, J.C. Izpisua Belmonte, Isolation and in vitro culture of primary cardiomyocytes from adult zebrafish hearts, Nature protocols 8(4) (2013) 800-9.

[12] A.N. Paradis, M.S. Gay, L. Zhang, Binucleation of cardiomyocytes: the transition from a proliferative to a terminally differentiated state, Drug Discov Today 19(5) (2014) 602-9.

[13] M.H. Soonpaa, K.K. Kim, L. Pajak, M. Franklin, L.J. Field, Cardiomyocyte DNA synthesis and binucleation during murine development, The American journal of physiology 271(5 Pt 2) (1996) H2183-9.

[14] P. Gan, M. Patterson, H.M. Sucov, Cardiomyocyte Polyploidy and Implications for Heart Regeneration, Annu Rev Physiol 82(1) (2020) 45-61.

[15] J.M. Gonzalez-Rosa, M. Sharpe, D. Field, M.H. Soonpaa, L.J. Field, C.E. Burns, C.G. Burns, Myocardial Polyploidization Creates a Barrier to Heart Regeneration in Zebrafish, Dev Cell 44(4) (2018) 433-446 e7.

[16] J. Piquereau, R. Ventura-Clapier, Maturation of Cardiac Energy Metabolism During Perinatal Development, Frontiers in physiology 9(959) (2018) 959.

[17] M. Writing Group, D. Mozaffarian, E.J. Benjamin, A.S. Go, D.K. Arnett, M.J. Blaha, M. Cushman, S.R. Das, S. de Ferranti, J.P. Despres, H.J. Fullerton, V.J. Howard, M.D. Huffman, C.R. Isasi, M.C. Jimenez, S.E. Judd, B.M. Kissela, J.H. Lichtman, L.D. Lisabeth, S. Liu, R.H. Mackey, D.J. Magid, D.K. McGuire, E.R. Mohler, 3rd, C.S. Moy, P. Muntner, M.E. Mussolino, K. Nasir, R.W. Neumar, G. Nichol, L. Palaniappan, D.K. Pandey, M.J. Reeves, C.J. Rodriguez, W. Rosamond, P.D. Sorlie, J. Stein, A. Towfighi, T.N. Turan, S.S. Virani, D. Woo, R.W. Yeh, M.B. Turner, C. American Heart Association Statistics, S. Stroke Statistics, Heart Disease and Stroke Statistics-2016 Update: A Report From the American Heart Association, Circulation 133(4) (2016) e38-360.

[18] B.J. Haubner, J. Schneider, U. Schweigmann, T. Schuetz, W. Dichtl, C. Velik-Salchner, J.I. Stein, J.M. Penninger, Functional Recovery of a Human Neonatal Heart After Severe Myocardial Infarction, Circ Res 118(2) (2016) 21621.

[19] B.J. Herdrich, E. Danzer, M.G. Davey, M. Allukian, V. Englefield, J.H. Gorman, 3rd, R.C. Gorman, K.W. Liechty, Regenerative healing following foetal myocardial infarction, European journal of cardio-thoracic surgery : official journal of the European Association for Cardio-thoracic Surgery 38(6) (2010) 691-8.

[20] L. Ye, G. D'Agostino, S.J. Loo, C.X. Wang, L.P. Su, S.H. Tan, G.Z. Tee, C.J. Pua, E.M. Pena, R.B. Cheng, W.C. Chen, D. Abdurrachim, J. Lalic, R.S. Tan, T.H. Lee, J. Zhang, S.A. Cook, Early Regenerative Capacity in the Porcine Heart, Circulation 138(24) (2018) 2798-2808.

[21] W. Zhu, E. Zhang, M. Zhao, Z. Chong, C. Fan, Y. Tang, J.D. Hunter, A.V. Borovjagin, G.P. Walcott, J.Y. Chen, G. Qin, J. Zhang, Regenerative Potential of Neonatal Porcine Hearts, Circulation 138(24) (2018) 2809-2816.

[22] C. Zogbi, A.E. Saturi de Carvalho, J.S. Nakamuta, M. Caceres Vde, S. Prando, M.C. Giorgi, C.E. Rochitte, J.C. Meneghetti, J.E. Krieger, Early postnatal rat ventricle resection leads to long-term preserved cardiac function despite tissue hypoperfusion, Physiol Rep 2(8) (2014) e12115.

[23] H. Sadek, E.N. Olson, Toward the Goal of Human Heart Regeneration, Cell stem cell 26(1) (2020) 7-16.

[24] N. Naqvi, M. Li, J.W. Calvert, T. Tejada, J.P. Lambert, J. Wu, S.H. Kesteven, S.R. Holman, T. Matsuda, J.D. Lovelock, W.W. Howard, S.E. lismaa, A.Y. Chan, B.H. Crawford, M.B. Wagner, D.I. Martin, D.J. Lefer, R.M. Graham, A. Husain, A proliferative burst during preadolescence establishes the final cardiomyocyte number, Cell 157(4) (2014) 795-807. 
[25] N. Naqvi, R. Singh, Siiri E. lismaa, M. Li, John W. Calvert, David I.K. Martin, Richard P. Harvey, Robert M. Graham, A. Husain, Cardiomyocytes Replicate and their Numbers Increase in Young Hearts, Cell 163(4) (2015) 783784.

[26] Mark H. Soonpaa, David C. Zebrowski, C. Platt, A. Rosenzweig, Felix B. Engel, Loren J. Field, Cardiomyocyte Cell-Cycle Activity during Preadolescence, Cell 163(4) (2015) 781-782.

[27] A. Garcia-Puig, J.L. Mosquera, S. Jimenez-Delgado, C. Garcia-Pastor, I. Jorba, D. Navajas, F. Canals, A. Raya, Proteomics Analysis of Extracellular Matrix Remodeling During Zebrafish Heart Regeneration, Mol Cell Proteomics 18(9) (2019) 1745-1755.

[28] A.C. Cardoso, N.T. Lam, J.J. Savla, Y. Nakada, A.H.M. Pereira, A. Elnwasany, I. Menendez-Montes, E.L. Ensley, U.B. Petric, G. Sharma, A.D. Sherry, C.R. Malloy, C. Khemtong, M.T. Kinter, W.L.W. Tan, C.G. Anene-Nzelu, R.S. Foo, N.U.N. Nguyen, S. Li, M.S. Ahmed, W.M. Elhelaly, S. Abdisalaam, A. Asaithamby, C. Xing, M. Kanchwala, G. Vale, K.M. Eckert, M.A. Mitsche, J.G. McDonald, J.A. Hill, L. Huang, P.W. Shaul, L.I. Szweda, H.A. Sadek, Mitochondrial Substrate Utilization Regulates Cardiomyocyte Cell Cycle Progression, Nat Metab 2(2) (2020) 167-178.

[29] D.C. Zebrowski, R. Becker, F.B. Engel, Towards regenerating the mammalian heart: challenges in evaluating experimentally induced adult mammalian cardiomyocyte proliferation, Am J Physiol Heart Circ Physiol 310(9) (2016) H1045-54. 
CHAPTER 5

Summary \& General Discussion 
The limited regenerative potential of the adult human heart contributes to the high morbimortality of cardiovascular diseases. In response to an ischemic event, cardiomyocytes are irreversibly lost via an apoptotic process and rapidly replaced by a fibrotic scar. Cardiac fibrosis prevents the rupture of the thinned ischemic ventricle but limits the propagation of the electric stimuli and contractile ability of the heart, which progressively lead to the development of heart failure. At the moment, clinical practice lacks a cardiomyocyte replacement therapy and heart transplantation continues to be the only long-term effective treatment for heart muscle restoration. Hence, a promising strategy to treat the heart following myocardial infarction could be to stimulate its endogenous regenerative capacity and prevent activation of pathological mechanisms.

In chapter 1, we revisited the cardiac regenerative capacity throughout phylogeny and ontogeny. Cardiac muscle renewal was firstly described in zebrafish [1] but was promptly replicated in other lower vertebrate species, like the axolotl [2] and newt [3], and later in mammalian hearts [4]. Comparison of events activated following injury in lower vertebrates and in neonatal murine hearts disclosed an evolutionarily conserved response encompassing: development of an inflammatory process, deposition of extracellular matrix, proliferation of pre-existing cardiomyocytes and functional recovery (reviewed in [5]).

However, in contrast to lower vertebrates, which exhibit lifelong cardiac regeneration, mammalian heart regeneration is restricted to a short period after birth [4]. In the transition between intra and extra-uterine life, mammalian hearts undergo dramatic alterations, defining the end of cardiac morphogenesis and the start of myocardial maturation, that significantly impact on cardiac regeneration [6-8]. In response to the increased oxygen pressure in the extra-uterine environment, postnatal cardiomyocytes undergo a metabolic shift from anaerobic glycolysis to mitochondrial oxidative phosphorylation. Reactive oxygen species, a by-product of mitochondrial respiration, were found to gradually increase throughout development and were shown to activate DNA damage response pathways, precluding cardiomyocyte proliferation. In contrast, hypoxic stimulus increased cardiomyocyte proliferation, which suggests that hypoxia signalling is an important regulator of cardiomyocyte proliferation [7].

This observation inspired us to revise the role of non-coding RNAs during hypoxia and their impact regulating endothelial cell and cardiomyocyte function in chapter 2 . Even though not leading to the generation of proteins, the majority of the non-coding genome is transcribed and is an important regulator of physiologic and pathologic mechanisms [9]. The evidence that non-coding RNAs are differently expressed in endothelial cells and cardiomyocytes after an ischemic injury suggests that they may regulate regeneration and neovascularization [10]. In this review we focused on different classes of non-coding RNAs (i.e. microRNAs (miRNAs/miRs), long non-coding RNAs (IncRNAs) and transcribed ultraconserved regions (T-UCRs)), and we identified several pieces of evidence supporting that ncRNAs play an epigenetic control of heart response to hypoxia by specific targeting signalling pathways and/or secreted molecules responsive to hypoxia. Hence, by focusing 
on differentially expressed non-coding RNAs between regenerative (neonatal) and nonregenerative periods (from $\mathrm{P} 7$ to adulthood), one may identify molecules promoting or hindering cardiomyocyte proliferation and neovascularization. Application of this knowledge for clinical purposes will require gene therapy namely for inhibition (e.g. antagomirs, locked nucleic acid (LNA) anti-miRs, small interfering RNAs (siRNAs) or short hairpin RNAs (shRNAs)) or upregulation (e.g. adeno-associated virus or miRNA precursors) of nucleic acids. Even though more studies are needed to consolidate the use of nucleic acid modulators as safe therapeutic alternatives, growing evidence strongly supports that noncoding RNAs are important regulators of cardiovascular diseases, making them promising targets for therapy.

\section{Apex resection activates regenerative and reparative mechanisms}

As previously discussed (chapter $\mathbf{1}$ ), the neonatal mammalian heart retains a transient regenerative capacity. Such findings were firstly reported in mice by observing the response of neonatal animals to cardiac injury. Importantly, this ability seems to be evolutionary conserved as different mammalian species (e.g. rat, sheep, pig, human) are also capable of regenerating cardiac muscle during the early postnatal period (reviewed in [5]). This illustrates how animal models of cardiac injury can be particularly useful, as the information drawn from them may also apply to the human heart. In fact, while the first models of cardiac injury did not fully recapitulate the pathophysiologic mechanisms of disease, there is a continuing effort to improve their translatability.

Interestingly, the generation of different cardiac injury models revealed some response variability to different types of injury. For instance, when subjected to cryoinjury, neonatal hearts undergo incomplete regeneration [11] whereas hearts challenged with myocardial infarction exhibit complete myocardial restoration [12]. In addition, hearts in which the apex was surgically removed showed different healing profiles across laboratories [4, 13]. In shear contrast with the pivotal work by Porrello et al. [4], Andersen and colleagues revealed that upon apical resection neonatal hearts lack regeneration, exhibiting instead extensive fibrosis and no cardiomyocyte replacement, resulting in the development of dilated cardiomyopathy $[13,14]$. Even though possible causes were debated [15-17], the reason of these discrepancies is still unclear, which hampers progress in the field. Furthermore, studies on neonatal cardiac regeneration focused either on functional assessment or on histopathological alterations, lacking therefore integration of the tissue response with functional outcome.

In chapter 3 we provide a detailed characterization of the neonatal apex resection injury model. By doing a systematic assessment of pathophysiological events occurring over 180 days after surgery we found that apical resection triggers both regenerative (e.g. cardiomyocyte proliferation and neovascularization) and reparative (e.g. cardiac fibrosis) mechanisms. Hence, our results are not compatible with full regeneration [4] nor exclusively with repair [13], showing an intermediate response which likely explains the controversy in the field. As previously discussed $[15,17]$, the surgery and experimental 
readout to assess regeneration are determinant for obtaining reliable results. Our injury size was comparable to previous publications, allowing further comparisons. By representatively sectioning the heart, we were able to detect a minor, yet permanent and consistent, deposition of fibrotic tissue in the injury site. This contrasts with a previous publication [4] and is likely explained by an incomplete histological sampling of the heart in that study. Nevertheless, a parallel longitudinal echocardiographic evaluation revealed that the cardiac fibrosis formed in the apical region had no deleterious impact on cardiac systolic nor diastolic function. In our setting, injured hearts showed permanent morphologic alterations when compared to sham controls (i.e. long-axis shortening and short-axis widening), which is in line with the observations by Andersen et al. [13, 14]. Yet, in contrast with these studies, the short-axis widening observed in our study results from chamber dilation and wall thickening which is not compatible with dilated cardiomyopathy. By profiling cardiac fibroblasts at different developmental stages and in response to injury, we found that neonatal cardiac fibroblasts display an intermediate phenotype between foetal and adult fibroblasts. Subsequent characterization of the main cardiac fibroblast subpopulations after injury showed heterogeneous transcriptomic profiles, supporting a ontogenic-signature-dependent biological role. While subsets predominantly found in the foetal hearts showed upregulated levels of genes associated with the production of extracellular matrix, neovascularization and secreted factors known to activate CM proliferation, those common in adult hearts showed activation of fibrotic genes. As such, these findings support that cardiac fibroblasts found in neonatal hearts are heterogenous and can simultaneously lead to fibrogenesis and regeneration. Considering that cardiomyocyte size was not increased in injured hearts, we were able to exclude the hypothesis that the wall thickening was a result of hypertrophic growth. Moreover, resorting to an ingenious stereological approach (described in detail in chapter 4), backed up by the quantification of cardiomyocytes expressing $\mathrm{pH} 3$, Aurora B and that incorporated EdU in a pulse-chase experiment, we were able to demonstrate that cardiomyocytes from resected hearts exhibited increased mitotic rates, accompanied by cytokinesis. Importantly, this strategy further allowed accurate estimation of total cardiomyocyte numbers in the left ventricle, which was essential to demonstrate that activation of cardiomyocyte proliferation in resected hearts overcompensated cardiomyocytes lost by resection. As such, the results reported in chapter 3 endorse apical resection injury model as particularly valuable for studies aiming to block fibrosis and to favour cardiomyocyte proliferation and functional cardiac regeneration.

\section{A new paradigm in the quantification of cardiomyocyte proliferation}

The identification of molecules with neomyogenic potential is a well rooted priority within cardiovascular community [18]. Cardiomyocyte proliferation is typically ascertained by detecting the expression of cell cycle markers (e.g. Ki67, PH3, Aurora B) and/or by quantification of thymidine analogues incorporation (e.g. BrdU, EdU) by cardiomyocytes. However, as discussed in chapter 1, postnatal cardiomyocytes undergo maturation, 
resulting in a cell cycle arrest. Besides the transcriptomic, proteomic, metabolomic, morphologic and mechanic changes, postnatal cardiomyocytes become multinucleated or polyploid due to incomplete karyokinesis or cytokinesis, respectively [19-21]. Importantly, binucleating cardiomyocytes, alike proliferating cardiomyocytes, express $\mathrm{Ki} 67, \mathrm{pH} 3$ and Aurora $\mathrm{B}$ and incorporate $\mathrm{EdU}$ and $\mathrm{BrdU}$, making these methodologies fallible to detect neomyogenesis [22]. Moreover, binucleation largely exceeds the number of proliferating cardiomyocytes, which might lead to a significant overestimation of proliferative events.

In chapter $\mathbf{4}$ we provide a detailed protocol to unequivocally quantify cardiomyocyte proliferation and/or numbers in the myocardium. In contrast to the aforementioned methodologies, our optimized protocol does not depend on spatial-temporal detection of proliferating cardiomyocytes but rather focuses on the outcome of these proliferative events. The combination of 3D imaging with stereology enabled estimation of the total number of cardiomyocytes, surpassing the limitation of traditional methods. As we determined the average number of nuclei per cardiomyocyte, we are able to distinguish cardiomyocyte proliferation from binucleation. In addition, we conduct a parallel EdU pulse-chase experiment allowing further estimation of replicating cardiomyocytes at any point in time.

Making use of this protocol, we confirmed that apical resection does elicit a regenerative response in neonatal murine hearts, characterized by proliferation of pre-existing cardiomyocytes (chapter 3). To further validate the potential of this protocol we measured the contribution of cardiomyocyte hyperplasia in the postnatal heart, by estimating the number of cardiomyocytes of P6 and P12 murine hearts (chapter 4). Due to the postnatal maturation of cardiomyocytes, cardiac growth during this period is considered to occur via the hypertrophic growth of cardiomyocytes. Interestingly, our approach revealed that P12 hearts had more cardiomyocytes in comparison to the younger P6 hearts, supporting a minor but significant role of postnatal cardiomyocyte hyperplasia in the definition of the total number of cardiomyocytes of the adult heart. In comparison to other methodologies, this protocol has the advantage of being compatible with the use of genetic constructs and does not require the detection of rare events (i.e. cardiomyocyte proliferation) and/or complex subcellular processes, such as midbody position and distance between daughter nuclei [23]. Even tough being labour intensive, the protocol presented herein is a powerful tool for studies aiming to investigate molecular mechanisms regulating cardiomyocyte proliferation.

\section{Concluding remarks and future perspectives}

In spite of the best effort of academics and clinicians, cardiovascular diseases are still responsible for roughly one third of all deaths worldwide [24]. Most of the available therapeutic approaches are focused in decelerating disease progression and do not replace nor functionally compensate for the lost cardiomyocytes.

In this thesis, we confirmed that apex resection activates regenerative and reparative mechanisms, making it suitable for the identification of molecules that regulate 
neomyogenesis, neovascularization and cardiac fibrosis, but also as a platform to test the therapeutic effect of anti-fibrotic and/or angiogenic or neomyogenic compounds.

This consisted on an important contribution to cardiovascular field as the value of this model had been questioned by conflicting results. In addition, this study exemplifies the need of standardization and comprehensive analysis of biological processes as neither of the previous reports $[4,13]$ was able to fully picture the response of the neonatal heart to apical resection.

This is particularly relevant as the generalization of hastily yet faulty techniques tends to produce deceiving results and has recently overshadowed the cardiovascular field. By the turn of the century, cells exhibiting stem cell-like properties were identified in the adult heart of rodents and humans, which was considered a promising breakthrough for cardiovascular medicine and even tested as a therapeutic intervention [25, 26]. Failure to replicate the results and validation using robust techniques, such as lineage tracing methods, revealed that cardiac progenitors do not differentiate into endothelial cells nor cardiomyocytes in vitro and in vivo, which discredited the concept and delayed progress in the field.

Herein, by performing an unparalleled characterization of the biological mechanisms activated following apical resection, we were able to demonstrate that this model is capable of activating cardiac regenerative mechanisms, despite local fibrosis.

In addition, we found a heterogenous population of cardiac fibroblasts within neonatal hearts which correlated with different transcriptomic signatures and potentially distinct biological functions. For long, cardiac fibroblasts were seen as the source of cardiac fibrosis and contributors to cardiac dysfunction. Our results challenge this view by demonstrating that neonatal murine cardiac fibroblasts exhibiting a foetal-like signature respond to injury by activating pro-regenerative genes. This is in line with previous results in vitro, where murine embryonic cardiac fibroblasts were shown to promote cardiomyocyte proliferation via paracrine action of secreted proteins, while the adult counterparts induced cardiomyocyte hypertrophy [27]. While further research should define the relationship between the molecular signature and biological function of cardiac fibroblasts the results reported in chapter 3 pave the way for new therapeutic strategies envisaging the modulation of cardiac fibroblasts towards an immature/pro-regenerative phenotype.

Discovery of neomyogenic molecules has been challenged by inaccurate techniques to assess cardiomyocyte proliferation. To tackle this issue, we developed an optimized protocol that couples stereology with 3D imaging of thick ventricle sections. This protocol allows estimation of cardiomyocyte numbers and proliferation and is valuable for those aiming to unveil novel mechanisms of cardiomyocyte proliferation and to evaluate the myogenic effect of candidate molecules. In a time in which most of literature elucidating on cardiomyocyte proliferation relies on misleading techniques, this protocol naturally opens the debate on how much of the cell cycle activity reported is truly leading to cardiomyocyte renewal, which can have enormous impact on current understanding of the neomyogenic process. As discussed in chapter 4, we anticipate that the stereological approach detailed in this thesis will constitute a valuable alternative or complementary approach for the 
quantification of cardiomyocyte proliferation. Stereology is particularly useful when hyperplasia is rare and therefore challenging to detect in space and time, but also when using genetically modified organisms as it does not rely on the addition of reporting alleles to label the progeny of dividing cells. The higher precision achieved by this methodology allows for a more efficient screening of neomyogenic/regenerative strategies. Furthermore, adoption of robust methodologies such as this, is expected to increase consistency between laboratories and minimize the reproducibility issues in the biomedical field.

The ultimate goal of regenerative medicine is to promote histo-functional restoration of diseased or injured tissues and reduce the need on organ transplantation. While some approaches are still being tested at the clinical and preclinical level, others have been approved by regulatory agencies and are now commercially available to tackle a number of pathologies (reviewed in [27]). In the heart, strategies to preserve and/or restore the myocardium after injury involve tissue engineering [28-30], cellular-based therapies [31, 32] and/or administration of factors or molecules promoting cardiomyocyte proliferation $[33,34]$.

While much still remains to be done [35], implementation of regenerative medicine therapies in the cardiovascular field will be of great importance to reduce the burden of cardiovascular diseases. The herein thesis brings the fields of regenerative medicine and cardiovascular research closer by validating the use of an injury model that had received some distrust and by detailing a protocol that allows unbiased quantification of cardiomyocyte proliferation. 


\section{References}

[1] K.D. Poss, L.G. Wilson, M.T. Keating, Heart regeneration in zebrafish, Science (New York, N.Y.) 298 (5601), 2002, 2188-2190, https://doi.org/10.1126/science.1077857

[2] I.L. Flink, Cell cycle reentry of ventricular and atrial cardiomyocytes and cells within the epicardium following amputation of the ventricular apex in the axolotl, Amblystoma mexicanum: confocal microscopic immunofluorescent image analysis of bromodeoxyuridine-labeled nuclei, Anatomy and embryology 205 (3), 2002, 235-244, https://doi.org/10.1007/s00429-002-0249-6

[3] F. Laube, M. Heister, C. Scholz, T. Borchardt, T. Braun, Re-programming of newt cardiomyocytes is induced by tissue regeneration, Journal of cell science 119 (22), 2006, 4719-4729, https://doi.org/10.1242/jcs.03252

[4] E.R. Porrello, A.I. Mahmoud, E. Simpson, J.A. Hill, J.A. Richardson, E.N. Olson, H.A. Sadek, Transient regenerative potential of the neonatal mouse heart, Science (New York, N.Y.) 331 (6020), 2011, 1078-1080, https://doi.org/10.1126/science.1200708

[5] E.L. Price, J.M. Vieira, P.R. Riley, Model organisms at the heart of regeneration, Disease Models \& Mechanisms 12 (10), 2019, dmm040691, https://doi.org/UNSP dmm040691

10.1242/dmm.040691

[6] J. Piquereau, R. Ventura-Clapier, Maturation of Cardiac Energy Metabolism During Perinatal Development, Frontiers in physiology 9 2018, 959, https://doi.org/10.3389/fphys.2018.00959

[7] B.N. Puente, W. Kimura, S.A. Muralidhar, J. Moon, J.F. Amatruda, K.L. Phelps, D. Grinsfelder, B.A. Rothermel, R. Chen, J.A. Garcia, C.X. Santos, S. Thet, E. Mori, M.T. Kinter, P.M. Rindler, S. Zacchigna, S. Mukherjee, D.J. Chen, A.I. Mahmoud, M. Giacca, P.S. Rabinovitch, A. Aroumougame, A.M. Shah, L.I. Szweda, H.A. Sadek, The oxygen-rich postnatal environment induces cardiomyocyte cell-cycle arrest through DNA damage response, Cell 157 (3), 2014 , 565-579, https://doi.org/10.1016/j.cell.2014.03.032

[8] G.A. Quaife-Ryan, C.B. Sim, M. Ziemann, A. Kaspi, H. Rafehi, M. Ramialison, A. El-Osta, J.E. Hudson, E.R. Porrello, Multicellular Transcriptional Analysis of Mammalian Heart Regeneration, Circulation 136 (12), 2017, 1123-1139, https://doi.org/10.1161/CIRCULATIONAHA.117.028252

[9] T.R. Mercer, M.E. Dinger, J.S. Mattick, Long non-coding RNAs: insights into functions, Nature reviews. Genetics 10 (3), 2009, 155-159, https://doi.org/10.1038/nrg2521

[10] R.P. Juni, R.C. Abreu, P.A. da Costa Martins, Regulation of microvascularization in heart failure - an endothelial cell, non-coding RNAs and exosome liaison, Noncoding RNA Res 2 (1), 2017, 45-55, https://doi.org/10.1016/j.ncrna.2017.01.001

[11] A. Darehzereshki, N. Rubin, L. Gamba, J. Kim, J. Fraser, Y. Huang, J. Billings, R. Mohammadzadeh, J. Wood, D. Warburton, V. Kaartinen, C.L. Lien, Differential regenerative capacity of neonatal mouse hearts after cryoinjury, Developmental biology 399 (1), 2015, 91-99, https://doi.org/10.1016/j.ydbio.2014.12.018

[12] B.J. Haubner, M. Adamowicz-Brice, S. Khadayate, V. Tiefenthaler, B. Metzler, T. Aitman, J.M. Penninger, Complete cardiac regeneration in a mouse model of myocardial infarction, Aging 4 (12), 2012, 966-977, https://doi.org/10.18632/aging.100526

[13] D.C. Andersen, S. Ganesalingam, C.H. Jensen, S.P. Sheikh, Do neonatal mouse hearts regenerate following heart apex resection?, Stem cell reports 2 (4), 2014, 406-413, https://doi.org/10.1016/j.stemcr.2014.02.008

[14] D.C. Andersen, C.H. Jensen, C. Baun, S. Hvidsten, D.C. Zebrowski, F.B. Engel, S.P. Sheikh, Persistent scarring and dilated cardiomyopathy suggest incomplete regeneration of the apex resected neonatal mouse myocardium-A 180 days follow up study, Journal of molecular and cellular cardiology 90 2016, 47-52, https://doi.org/10.1016/j.yjmcc.2015.11.031

[15] D.C. Andersen, C.H. Jensen, S.P. Sheikh, Response to Sadek et al. and Kotlikoff et al, Stem cell reports 3 (1), 2014, 3-4, https://doi.org/10.1016/j.stemcr.2014.06.011

[16] M.I. Kotlikoff, M. Hesse, B.K. Fleischmann, Comment on "Do neonatal mouse hearts regenerate following heart apex resection"?, Stem cell reports 3 (1), 2014, 2, https://doi.org/10.1016/j.stemcr.2014.06.010

[17] H.A. Sadek, J.F. Martin, J.K. Takeuchi, J. Leor, Y. Nie, M. Giacca, R.T. Lee, Multi-investigator letter on reproducibility of neonatal heart regeneration following apical resection, Stem cell reports 3 (1), 2014, 1, https://doi.org/10.1016/j.stemcr.2014.06.009

[18] S.M. Payan, F. Hubert, F. Rochais, Cardiomyocyte proliferation, a target for cardiac regeneration, Biochim Biophys Acta Mol Cell Res 2019, https://doi.org/10.1016/j.bbamcr.2019.03.008

[19] M.A. Laflamme, C.E. Murry, Heart regeneration, Nature 473 (7347), 2011, 326-335, https://doi.org/10.1038/nature10147

[20] A.N. Paradis, M.S. Gay, L. Zhang, Binucleation of cardiomyocytes: the transition from a proliferative to a terminally differentiated state, Drug Discov Today $19 \quad$ (5), 2014, 602-609, https://doi.org/10.1016/j.drudis.2013.10.019 
[21] M.H. Soonpaa, K.K. Kim, L. Pajak, M. Franklin, L.J. Field, Cardiomyocyte DNA synthesis and binucleation during murine development, The American journal of physiology 271 (5 Pt 2), 1996, H2183-2189, https://doi.org/10.1152/ajpheart.1996.271.5.H2183

[22] D.C. Zebrowski, R. Becker, F.B. Engel, Towards regenerating the mammalian heart: challenges in evaluating experimentally induced adult mammalian cardiomyocyte proliferation, American journal of physiology. Heart and circulatory physiology 310 (9), 2016, H1045-1054, https://doi.org/10.1152/ajpheart.00697.2015

[23] M. Hesse, M. Doengi, A. Becker, K. Kimura, N. Voeltz, V. Stein, B.K. Fleischmann, Midbody Positioning and Distance Between Daughter Nuclei Enable Unequivocal Identification of Cardiomyocyte Cell Division in Mice, Circulation research 123 (9), 2018, 1039-1052, https://doi.org/10.1161/CIRCRESAHA.118.312792

[24] D. Mozaffarian, E.J. Benjamin, A.S. Go, D.K. Arnett, M.J. Blaha, M. Cushman, S.R. Das, S. de Ferranti, J.P. Despres, H.J. Fullerton, V.J. Howard, M.D. Huffman, C.R. Isasi, M.C. Jimenez, S.E. Judd, B.M. Kissela, J.H. Lichtman, L.D. Lisabeth, S. Liu, R.H. Mackey, D.J. Magid, D.K. McGuire, E.R. Mohler, 3rd, C.S. Moy, P. Muntner, M.E. Mussolino, K. Nasir, R.W. Neumar, G. Nichol, L. Palaniappan, D.K. Pandey, M.J. Reeves, C.J. Rodriguez, W. Rosamond, P.D. Sorlie, J. Stein, A. Towfighi, T.N. Turan, S.S. Virani, D. Woo, R.W. Yeh, M.B. Turner, C. American Heart Association Statistics, S. Stroke Statistics, Heart Disease and Stroke Statistics-2016 Update: A Report From the American Heart Association, Circulation $133 \quad$ (4), 2016, e38-360, https://doi.org/10.1161/CIR.0000000000000350

[25] R. Bolli, A.R. Chugh, D. D'Amario, J.H. Loughran, M.F. Stoddard, S. Ikram, G.M. Beache, S.G. Wagner, A. Leri, T. Hosoda, F. Sanada, J.B. Elmore, P. Goichberg, D. Cappetta, N.K. Solankhi, I. Fahsah, D.G. Rokosh, M.S. Slaughter, J. Kajstura, P. Anversa, Cardiac stem cells in patients with ischaemic cardiomyopathy (SCIPIO): initial results of a randomised phase 1 trial, Lancet (London, England) 378 (9806), 2011, 1847-1857, https://doi.org/10.1016/S01406736(11)61590-0

[26] R.R. Makkar, R.R. Smith, K. Cheng, K. Malliaras, L.E. Thomson, D. Berman, L.S. Czer, L. Marban, A. Mendizabal, P.V. Johnston, S.D. Russell, K.H. Schuleri, A.C. Lardo, G. Gerstenblith, E. Marban, Intracoronary cardiospherederived cells for heart regeneration after myocardial infarction (CADUCEUS): a prospective, randomised phase 1 trial, Lancet (London, England) 379 (9819), 2012, 895-904, https://doi.org/10.1016/S0140-6736(12)60195-0

[27] M. Ieda, T. Tsuchihashi, K.N. Ivey, R.S. Ross, T.T. Hong, R.M. Shaw, D. Srivastava, Cardiac fibroblasts regulate myocardial proliferation through beta1 integrin signaling, Dev Cell 16 (2), 2009, 233-244, https://doi.org/10.1016/j.devcel.2008.12.007

[28] A.S. Mao, D.J. Mooney, Regenerative medicine: Current therapies and future directions, Proc Natl Acad Sci U S A 112 (47), 2015, 14452-14459, https://doi.org/10.1073/pnas.1508520112

[29] E. Cambria, F.S. Pasqualini, P. Wolint, J. Gunter, J. Steiger, A. Bopp, S.P. Hoerstrup, M.Y. Emmert, Translational cardiac stem cell therapy: advancing from first-generation to next-generation cell types, NPJ Regen Med 2 (1), 2017, 17, https://doi.org/10.1038/s41536-017-0024-1

[30] A.C. Silva, S.C. Rodrigues, J. Caldeira, A.M. Nunes, V. Sampaio-Pinto, T.P. Resende, M.J. Oliveira, M.A. Barbosa, S. Thorsteinsdottir, D.S. Nascimento, O.P. Pinto-do, Three-dimensional scaffolds of fetal decellularized hearts exhibit enhanced potential to support cardiac cells in comparison to the adult, Biomaterials 104 2016, 52-64, https://doi.org/10.1016/j.biomaterials.2016.06.062

[31] Y. Song, C. Zhang, J. Zhang, N. Sun, K. Huang, H. Li, Z. Wang, K. Huang, L. Wang, An injectable silk sericin hydrogel promotes cardiac functional recovery after ischemic myocardial infarction, Acta Biomater 41 2016, 210223, https://doi.org/10.1016/j.actbio.2016.05.039

[32] Y.W. Liu, B. Chen, X. Yang, J.A. Fugate, F.A. Kalucki, A. Futakuchi-Tsuchida, L. Couture, K.W. Vogel, C.A. Astley, A. Baldessari, J. Ogle, C.W. Don, Z.L. Steinberg, S.P. Seslar, S.A. Tuck, H. Tsuchida, A.V. Naumova, S.K. Dupras, M.S. Lyu, J. Lee, D.W. Hailey, H. Reinecke, L. Pabon, B.H. Fryer, W.R. MacLellan, R.S. Thies, C.E. Murry, Human embryonic stem cell-derived cardiomyocytes restore function in infarcted hearts of non-human primates, Nat Biotechnol 36 (7), 2018, 597-605, https://doi.org/10.1038/nbt.4162

[33] Y. Shiba, T. Gomibuchi, T. Seto, Y. Wada, H. Ichimura, Y. Tanaka, T. Ogasawara, K. Okada, N. Shiba, K. Sakamoto, D. Ido, T. Shiina, M. Ohkura, J. Nakai, N. Uno, Y. Kazuki, M. Oshimura, I. Minami, U. Ikeda, Allogeneic transplantation of iPS cell-derived cardiomyocytes regenerates primate hearts, Nature 538 (7625), 2016, 388-391, https://doi.org/10.1038/nature19815

[34] K. Gabisonia, G. Prosdocimo, G.D. Aquaro, L. Carlucci, L. Zentilin, I. Secco, H. Ali, L. Braga, N. Gorgodze, F. Bernini, S. Burchielli, C. Collesi, L. Zandona, G. Sinagra, M. Piacenti, S. Zacchigna, R. Bussani, F.A. Recchia, M. Giacca, MicroRNA therapy stimulates uncontrolled cardiac repair after myocardial infarction in pigs, Nature 569 (7756), 2019, 418-422, https://doi.org/10.1038/s41586-019-1191-6

[35] T.M.A. Mohamed, Y.S. Ang, E. Radzinsky, P. Zhou, Y. Huang, A. Elfenbein, A. Foley, S. Magnitsky, D. Srivastava, Regulation of Cell Cycle to Stimulate Adult Cardiomyocyte Proliferation and Cardiac Regeneration, Cell 173 (1), 2018, 104-116 e112, https://doi.org/10.1016/j.cell.2018.02.014 
[36] F. Fernandez-Aviles, R. Sanz-Ruiz, A.M. Climent, L. Badimon, R. Bolli, D. Charron, V. Fuster, S. Janssens, J. Kastrup, H.S. Kim, T.F. Luscher, J.F. Martin, P. Menasche, R.D. Simari, G.W. Stone, A. Terzic, J.T. Willerson, J.C. Wu, T.W. Group, C. Authors/Task Force Members, S. Basic Research, S. Translational Research, S. Challenges of Cardiovascular Regenerative Medicine, S. Tissue Engineering, N.T. Delivery, S. Assessment, S. Clinical Trials, Regulatory, s. funding strategies, N.T. Delivery, S. Assessment, Global position paper on cardiovascular regenerative medicine, European heart journal 38 (33), 2017, 2532-2546, https://doi.org/10.1093/eurheartj/ehx248 
Impact 


\section{Socio-Economic Impact}

Reports from the World Health Organization show that cardiovascular diseases continue to lead the causes of death in industrialized countries. Still, the ongoing globalization and adoption of a westernized life style also in lower-income countries, coupled to a reduced access to cardiovascular drugs and treatments, is contributing to an increased incidence of this group of diseases in emergent economies.

Among cardiovascular diseases, ischemic heart disease is particularly detrimental and a major onset of heart failure. In addition to the harmful effect on patient's health status and longevity, cardiovascular diseases impose a severe socio-economic burden. Recent improvement on the early detection of cardiovascular events and enhancement of treatments, have allowed patients with cardiovascular diseases to live longer while being carriers of diseases that would have been lethal during the last century. This increased longevity comes with increased costs associated to specialized health care and frequent hospitalizations.

At a time in which most of the available drugs focus on disease prevention or progression, much remains to be done towards the implementation of truly curative therapies. This is one of the current challenges of the cardiovascular community. The herein thesis contributes to the relatively new field of cardiovascular regenerative medicine. We specifically focused on validating a model that had gathered some negative attention from conflicting results. Furthermore, we implemented an unbiased protocol to ascertain cardiomyocyte proliferation, a major hallmark of cardiac regeneration.

\section{Target Groups}

The work reported in this thesis is of pivotal importance for several stakeholders. In chapter 3 we focused on the response developed by neonatal hearts following apical resection. This model was initially proposed as an important tool to investigate regenerative mechanisms and to test the pro-regenerative effect of candidate molecules, but has been recently involved in some controversy due to contrasting results reported in different publications. We performed an exhaustive characterization of the mechanisms elicited by apical resection up to 180 days after injury. We observed that both reparative and regenerative processes were activated which likely explains the contrasting results reported in the literature. As such, we were able to confirm that this model is valuable for those willing to study the regenerative properties of the neonatal mammalian heart.

Because cardiomyocyte proliferation is technically challenging to quantify, in chapter $\mathbf{4}$ we report an optimized protocol that is able to precisely estimate the number of cardiomyocytes in the heart. This protocol combines the power of tridimensional confocal imaging with stereology and is far more sensitive that the traditional methodologies in use. Although this approach is considerably more labor-intensive than other methods available, 
it provides a robust and unbiased result, which will be useful for determining the neomyogenic potential of candidate molecules.

A direct beneficiary of the data provided in this thesis is the scientific community, especially the cardiovascular community. In chapter 3, we convincingly demonstrate that the neonatal murine heart is able to mount a regenerative response after apical resection, which was still an open question in the field. We observed that injured hearts showed increased cardiomyocyte proliferation and neovascularization together with cardiac fibrosis in the innermost region of the apex, which did not compromise cardiac function. Such results reveal a partial overlap with previously published results but are neither compatible with full regeneration or solely with repair. This highlights that the surgical procedure and the criteria to assess heart's response to injury should be taken seriously and standardization is desirable. Furthermore, we provide evidence that cardiac fibroblasts play a dual role in the injured neonatal heart and that immature subsets can assist on cardiac regeneration, which can be explored for therapeutic purposes. In chapter $\mathbf{4}$, we have another example of a major contribution to the cardiovascular field. We disclosed a protocol that allows quantification of cardiomyocyte proliferation and/or number in the myocardium. Until now, progress in the field has been hampered by faulty techniques so a methodology that would allow unbiased quantification of cardiomyocytes was needed. We expect that this protocol will contribute to the discovery of molecules with neomyogenic potential.

As briefly discussed in chapter 5, the work in the herein thesis illustrates that the use of robust methodologies rather than fast yet faulty techniques, ensures a better and more reliable understanding of biological processes. In fact, the recent past is plentiful of studies from which candidate therapies have arisen and then proved to be inefficient in clinical trials, resulting in significant expenditure of time and money. Hence, the better control and standardization of methodologies at pre-clinical level, as defended in this thesis, will lead to a wiser investment in the finding of truly effective therapies.

In addition to the scientific community, pharmaceutical companies are also a beneficiary of the work reported herein. These companies are focusing on developing new products that can mitigate cardiovascular diseases. In the phase of developing new therapies that envisage cardiac regeneration (e.g. pro-angiogenic or neomyogenic factors), neonatal apex resection injury, reported in chapter $\mathbf{3}$, can be used to assess their impact at a pre-clinical level. Moreover, induction of cardiomyocyte proliferation can be quantified using the methodology reported in chapter 4.

Lastly, another important target of the work reported in this document are patients and general population. Patients will personally benefit from the development therapies that envisage cardiac muscle restoration, as this will allow them to live longer and better quality lives. In parallel, the decline of cardiovascular diseases, due to the development of curative therapies, will lead to less money spent in hospitalizations and health care, reducing the economic burden of these diseases. 


\section{Activities and Dissemination}

Dissemination of knowledge is an important part of the scientific activity. Knowledge transfer allows new hypothesis to be developed and tested and add increasing complexity to the current state of understanding. To do so, the data reported in this thesis was presented and discussed in several international conferences, including meetings from the European Society of Cardiology, Keystone and International Society for Heart Research. To make our work available to the scientific and general population, scientific outcomes have been submitted and published in international, peer-reviewed journals, including in the form of open-access. 
Abstract in Portuguese 
As doenças cardiovasculares constituem a principal causa de morte em países de médio e alto rendimento. Esta realidade deve-se, em parte, à baixa capacidade regenerativa do coração adulto humano que, em resposta a um enfarte do miocárdio, desenvolve uma resposta reparativa. A isquemia causada pelo enfarte leva à morte de cardiomiócitos e estes são gradualmente substituídos por um tecido cicatricial não contráctil, o que muitas vezes culmina na condição de falência cardíaca. Num período em que a única terapia eficaz a longo prazo para a restauração de cardiomiócitos é o transplante cardíaco, o desenvolvimento de novas alterativas terapêuticas é imperativo.

A observação de que murganhos neonatais são capazes de regenerar o seu miocárdio, definiu uma janela de oportunidade para a criação de novas terapias, que têm como base a indução da proliferação de cardiomiócitos, particularmente relevantes para o tratamento do coração adulto. No entanto, a divulgação de resultados contrastantes sobre a capacidade regenerativa de corações neonatais e o facto da proliferação de cardiomiócitos ser tecnicamente difícil de avaliar, tem impedido o avanço do conhecimento.

O trabalho reportado nesta tese contribui para a área cardiovascular, ao fazer uma caracterização detalhada da resposta de um coração neonatal de murganho à remoção cirúrgica do seu ápice. Ao avaliarmos longitudinalmente os mecanismos ativados durante os seis meses subsequentes à lesão, observámos que a ressecção induz mecanismos regenerativos (proliferação de cardiomiócitos e angiogénese) e mecanismos reparativos (fibrose cardíaca), o que potencialmente explica a controvérsia associada a este modelo.

Adicionalmente, detalhamos um protocolo que permite a avaliação rigorosa da proliferação de cardiomiócitos. Este protocolo combina a apreciação tridimensional do coração com estereologia, o que permite estimar o número total de cardiomiócitos no miocárdio. Em contraste com outros protocolos disponíveis, este não depende da observação espáciotemporal do evento proliferativo, mas quantifica o número de células resultante do mesmo. Esta abordagem permite ainda avaliar a nucleação de cardiomiócitos, o que é particularmente relevante, pois permite distinguir a proliferação da binucleação de cardiomiócitos.

Em suma, o trabalho reportado nesta tese aproxima as áreas da medicina regenerativa e da cardiologia ao validar o uso do modelo cirúrgico da remoção do ápice cardíaco para a descoberta de mecanismos regenerativos. Esta tese coloca ainda à disposição da comunidade científica um protocolo que permite avaliar, de forma precisa e imparcial, o potencial neomiogénico de novas moléculas e tratamentos. 
Acknowledgements 
As every good story, my PhD was marked by some plot twists. As every good story, I reach the end extremely happy and marked by a significant amount of people.

When I started this adventure in 2016, I was certain that I wanted to have the experience of doing research abroad. I wanted to step out of my comfort zone, live in a different country and experience different cultures. I did not think that 5 years later I was going to have the opportunity of defending my thesis in Maastricht and have the honor of receiving a double PhD degree.

During this process I grew professionally, I expanded my network, showed myself to new audiences and had the opportunity to work in an international group and department. I also matured personally, refined my character and understood what are my true motivations in life and what I would like to achieve.

This journey would not have been possible without the precious help of some people.

Diana, even though my PhD lasted for five years, we know each other for almost eight! You were/are a great mentor and everything I know I owe it specially to you - as Perpétua likes to say: "I remember her of you"! It was a pleasure to "grow" with you and I hope I also taught you something in return. I will always see you as an example of perseverance, determination and hard-work. Thank you for granting me your friendship.

Perpétua, you are a true example for life. I admire your creativity and motivation. You are a person with endless knowledge and a thousand talents. With you I understood the meaning of fairness, of never taking shortcuts and of fighting for what one deserves. You are a great leader, as you set the example but not the boundaries. You let people learn with their mistakes and "fly" when they are ready. Thank you for your example and support.

Paula, my Dutch yet Portuguese supervisor! The time we shared together was shorter but not what I learned from you. You are an example of success. You contribute to share the message that our small yet great country is full of talented and hard-working people. With you I learned that we should be ready to recognize and take an opportunity when it presents itself to us.

Leon, you are not part of the "official" supervising team but, to me, you are no less than a supervisor. I am grateful for the discussions and scientific input I got from you but also for the career advices. You are a talent-seeker and I appreciate having the opportunity of being part of your group.

To the "old guard" of PPO's lab (Tatiana, Ana Freire, Mariana, Francisca Araújo, Diogo, Ana Silva) I want to express my deep gratitude. As a new-comer you made me feel at home and I have learned so much from you.

To my Portuguese PhD mates (Tiago and Francisca (Chiquinha)), I want to thank your presence and friendship! At first, Tiago saved me from the "sea" of X chromosomes but rapidly Francisca arrived and conquered a spot in the "gang". Both of you have an enormous ambition and motivation and it was a pleasure doing this journey right beside you and see 
each and single one of us fly away and pursue our own goals. I will always keep in my heart the long hours in the lab but also the long hours outside it!

To PPO's "freshman" (Elsa, Márcia, Rita, Daniel*), I shared more time with some than others but I wish you have a great PhD and have the same or more satisfaction I had during mine. As once you belong to this "family", you really never leave, Daniel appears here. All the best for your PhD in Czech Republic. Finally, a special thanks to Elsa whom, in all her extravagance, showed to be a true and precious friend.

To our "eternal" visiting researcher, Francisco, I have to thank for the fruitful collaborations, the long hours spent in the animal facility, the explanations about cardiac physiology and, not less important, the animated dinners!

To all the INEBians and I3Sians that I had the pleasure to have by my side throughout this journey, I want to express my deep gratitude.

In the "Maastricht family", I want to start with Lara, Andrea and Marida. One could think that moving to a new country could be difficult. The truth is that you received me with open arms and I felt like I had never left home. Now that life made us move in different ways, and we are physically apart, I feel like nothing has changed. I gained friends for life and I am truly grateful for that!

Lara, thank you for your unconditional support when things did not go so well and for cherishing the moments in which they did! Thanks for all the dinners and for hosting us in Aldenhofpark.

Andrea, thank you for showing me how to put things in perspective and take a positive lesson of every single thing. Thanks for all the conversations, runs, travels.

Marida, thank you for all the laughs we shared and the positive vibe you put in everything you do. Thanks for hosting and show us your beautiful Puglia!

Raquel, my Portuguese "younger sister" that kept the days always busy with infinite discussions about everything $:$. . Good luck for the PhD and future endeavors!

Indira, the girl from the island, that constantly remembered me the importance of what we do. I will always remember the holiday in Puglia and the windsurf lessons!

Nicolò, thanks for your unending energy and charisma. I wish you all the best!

Claudia and Robin, I group you because you are two of the most hard-working persons I have ever met. Thanks for setting the example and I am sure you will achieve everything you wish for!

Jana, the go-to person during immersions in bioinformatics but, most importantly, my pseudo-housemate! Thanks for all the discussions and explanations. 
Martina, I will never forget our congresses together and all the scientific discussions. Thanks for all your feedback and for showing the recipe to be a great researcher.

Ricardo and Andreia, it was a pity that our time in Maastricht was never overlapping. Yet, I got to understand that you excel on what you do and I learned a lot from your input in lab meetings, journal clubs, etc. Hope to meet you soon and good luck for the rest of the PhD.

Zenab, the time I spent with you was few! Thanks for your kindness and for the nice conversations when visiting us in the lab.

Deepak, Giulia and Joana, the time with you was also very short! I wish good fortune for your PhDs and for everything you want to achieve.

To Servé, Roel and Sandrine, thanks for your help and to make things work in the lab.

To Lauren, our engineer, singer, sommelier, I want to thank you for your contagious positive attitude. Thanks for the dinners and glasses of wine we shared but most importantly for all the conversations we had!

For all the remaining people, closer or in distance, that were not addressed in particular. I want to thank you for your friendship and support. If the completion of this $\mathrm{PhD}$ is possible, it is also due to the interaction, experiences, discussions and lessons I learned with every single one and made me up to this task.

To my Family, I want to thank them for their unconditional support. For the work they put to create the conditions that allowed me to follow my dreams, while asking nothing in return. I am very lucky and grateful for having you by my side and the completion of this $\mathrm{PhD}$ is shared with you.

Finally, I want to thank Cristina. Your presence brought the tranquility and renovated strengths when I had to face hard times and the inspiration and joy to celebrate the good ones. I am truly grateful in having you by my side and to share this moment with you. 


\section{Biography}

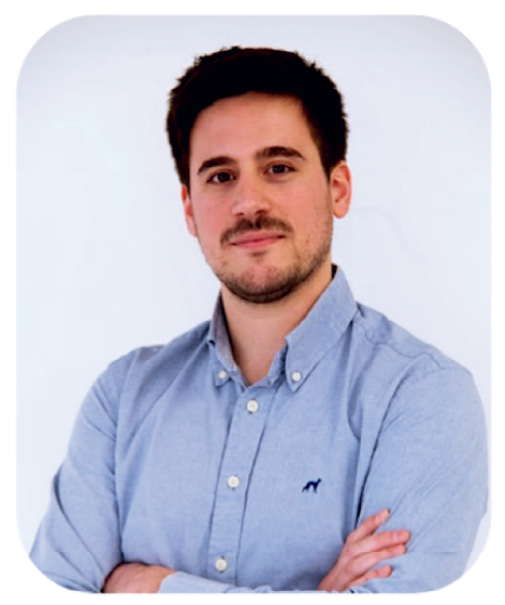

Vasco Sampaio Pinto was born the $2^{\text {nd }}$ of July 1991 in Vila Franca de Xira (Lisbon, Portugal). After graduating in Biology in 2012 he joined the Evolutionary and Developmental Biology Master program from Faculty of Sciences of University of Lisbon. The close connection between embryonic development and regeneration led him to join the team of Perpétua Pinto-do-Ó at Instituto Nacional de Engenharia Biomédica (Porto, Portugal), where he performed his master thesis. In 2015 he was awarded a PhD individual fellowship from FCT (Fundação para a Ciência e Tecnologia [Foundation for Science and Technology]) and enrolled on a joint PhD between the Universities of Porto (Instituto de Ciências Biomédias Abel Salazar) and Maastricht (CARIM). During his PhD he has been focused on evaluating the response of neonatal murine hearts to injury and on exploring the regenerative and reparative mechanisms activated upon cardiac damage. Furthermore, the lack of reliable methodologies to quantify cardiomyocyte proliferation motivated Vasco to develop a protocol based on stereology that allows an unbiased evaluation of neomyogenesis.

\section{List of Publications}

\section{Journal Publications}

1) Vonhögen IGC*, Dirkx E*, Murri M, el Azzouzi H, Corbeels K, Salic K, SAMPAIO-PINTO V, Olieslagers S, van der Schueren B, de Bruin A, da Costa Martins PA, De Windt LJ. Brain-specific miR-216a ablation impairs high-fat diet-induced obesity and insulin resistance (Scientific Reports, submitted).

2) Silva ED*, Tomé I*, Vasques-Nóvoa F, Conceição G, Silva A, Barros A, Gonçalves A, Caetano C, Sousa D, Silva D, Gonçalves N, SAMPAIO-PINTO V, Gonçalves A, LeiteMoreira A, Falcão-Pires I, Pitrez I, Pinto-do-Ó P, Santos SG, Ferreira L, Nascimento DS. Pharmacological clearance of senescent cells with Navitoclax reverses HFpEF hallmarks by decreasing inflammation, endothelial dysfunction and cardiac fibrosis (Nature Aging, in revision).

3) Ottaviani L*, Juni RP*, Sansonetti M, SAMPAIO-PINTO V, Halkein J, Hegenbarth JC, Ring N, Knoops K, Kocken JMM, el Azzouzi H, Rühle F, Olieslagers S, Braga L, Stoll M, Nascimento DS, de Windt $\mathrm{L}$, da Costa-Martins PA. Intercellular transfer of miR-200c$3 p$ impairs the angiogenic capacity of cardiac endothelial cells (Science Translational Medicine, in revision). 
4) SAMPAIO-PINTO V*, Janssen J*, Chirico N, Serra M, Alves PM, Doevendans PA, Voets IK, Sluijter JPG, van Laake LW, van Mil A. A roadmap to cardiac tissue-engineered construct preservation. * - equal contribution. (Advanced Materials, accepted).

5) SAMPAIO-PINTO V, Ruiz-Villalba A, Nascimento DS, Pérez-Pomares JM. Bone marrow contribution to the heart from development to adulthood, Semin Cell Dev Biol. 2021 Apr;112:16-26.

6) Tas RP*, SAMPAIO-PINTO V*, Wennekes T, van Laake LW, Voets IK. From the freezer to the clinic: Antifreeze proteins in the preservation of cells, tissues, and organs. EMBO Rep. 2021 Mar 3;22(3):e52162.

7) Laundos TL, Vasques-Nóvoa F, Gomes RN, SAMPAIO-PINTO V, Cruz P, Cruz H, Santos JM, Barcia RN, Pinto-do-Ó P, Nascimento DS. Consistent long-term therapeutic efficacy of human umbilical cord matrix derived mesenchymal stromal cells after myocardial infarction despite individual differences and transient engraftment. Front Cell Dev Biol. 2021 Feb 4;9:624601.

8) SAMPAIO-PINTO V, Silva ED, Laundos TL, da Costa-Martins PA, Pinto-do-Ó P, Nascimento DS. Stereological estimation of cardiomyocyte number and proliferation, Methods. 2020 Jun 27:S1046-2023(19)30312-3.

9) Peters M, SAMPAIO-PINTO V, da Costa-Martins PA. Non-coding RNAs in endothelial cell signaling and hypoxia during cardiac regeneration, Biochim Biophys Acta Mol Cell Res. 2020 Mar;1867(3):118515.

10) Silva C, SAMPAIO-PINTO V, Andrade $S$, Rodrigues I, Costa R, Guerreiro S, Carvalho E, Pinto-do-Ó P, Nascimento DS, Soares R. Establishing a Link between Endothelial cell Metabolism and Vascular Behaviour in a Type 1 Diabetes Mouse Model, Cell Physiol Biochem. 2019;52(3):503-516.

11) Raso $A^{*}$, Dirkx E*, Philippen LE*, Fernandez-Celis A, De Majo F, SAMPAIO-PINTO V, Sansonetti M, Juni R, el Azzouzi H, Calore M, Bitsch N, Olieslagers S, Oerlemans MIFJ, Huibers MM, de Weger RA, Reckman YJ, Pinto YM, Zentilin L, Zacchigna S, Giacca M, da Costa Martins PA, López- Andrés N, De Windt L, Therapeutic delivery of miR-148a suppresses ventricular dilation in heart failure, Mol Ther. 2019 Mar 6;27(3):584-599.

12) SAMPAIO-PINTO V, Rodrigues $S$, Laundos TL, Silva ED, Vasques-Nóvoa $F$, Silva AC, Cerqueira RJ, Resende TP, Pianca N, Leite-Moreira A, D'Uva G, Thorsteinsdóttir S, Pinto-do-Ó P*, Nascimento DS*. Neonatal Apex Resection Triggers Cardiomyocyte Proliferation, Neovascularization and Functional Recovery in Spite of Local Fibrosis, Stem Cell Reports. 2018 Mar 13;10(3):860-874. 
13) Silva AC, Rodrigues SC*, Caldeira J*, Nunes AM*, SAMPAIO-PINTO V, Resende TP, Oliveira MJ, Barbosa MA, Thorsteinsdóttir S, Nascimento DS\$, Pinto-do-Ó P\$. Threedimensional scaffolds of decellularized fetal hearts exhibit enhanced potential to support cardiac cells in vitro, Biomaterials. 2016 Oct;104:52-64.

\section{Preprints}

1) Raso A, Dirkx E, SAMPAIO-PINTO V, el Azzouzi H, Cubero RJ, Sorensen DW, Ottaviani L, Olieslagers S, Huibers MM, de Weger R, Siddiqi S, Moimas S, Torrini C, Zentillin L, Braga L, da Costa-Martins PA, van Berlo JH, Zacchigna S, Giacca M, de Windt LJ. A microRNA program controls the transition of cardiomyocyte hyperplasia to hypertrophy and stimulates mammalian cardiac regeneration. bioRxiv 2021.02.25.432908.

\section{Book Chapters}

1) SAMPAIO-PINTO V, Silva AC, Pinto-do-Ó P, Nascimento DS. Cardiac regeneration and repair: from mechanisms to therapeutic strategies. Concepts and Applications of Stem Cell Biology. Springer International Publishing, pp. 187-211.
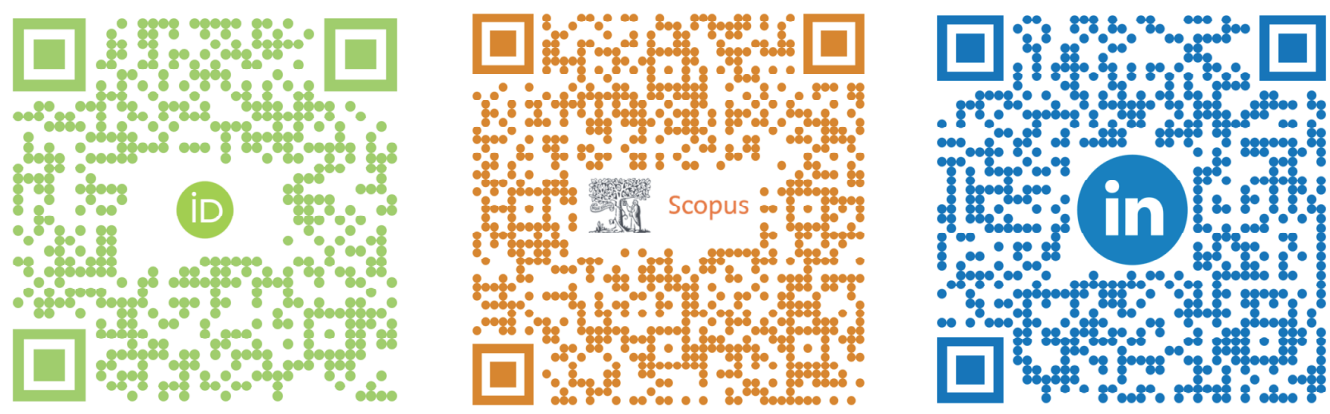